Annals of Mathematics, 154 (2001), 1-103

\title{
Deviation of ergodic averages for area-preserving flows on surfaces of higher genus
}

\author{
By Giovanni Forni*
}

Table of Contents

Introduction

1. The Kontsevich-Zorich cocycle

2. Variational formulas

3. A lower bound for the second Lyapunov exponent

4. The determinant locus

5. The Kontsevich-Zorich formula revisited and other formulas for the Lyapunov exponents

6. Basic currents for measured foliations

6.1. Basic currents and invariant distributions

6.2. Weighted Sobolev spaces of currents

6.3. A geometric estimate of the Poincaré constant

7. The structure of the space of basic currents of finite order

7.1. Basic currents with non-vanishing cohomology class

7.2. Basic currents with vanishing cohomology class

8. The non-uniform hyperbolicity of the Kontsevich-Zorich cocycle and an application to currents

8.1. The stable and unstable sub-bundles of the Kontsevich-Zorich cocycle as bundles of basic currents

8.2. The Kontsevich-Zorich cocycle is non-uniformly hyperbolic

8.3. The Oseledec's theorem for the bundle of closed currents of order 1

* This paper rests on the work of several mathematicians, H. Masur, J. Smillie, W. Veech and A. Zorich among them, and it was strongly inspired by the work of A. Zorich and M. KontsevichA. Zorich. I wish to thank particularly A. Eskin, J. Smillie and A. Zorich for their interest during the slow progress of this work and for discussing with me their ideas on the subject. I am grateful to R. Gunning for his encouragement and his enthusiasm during several discussions on the content of Section 4, which improved very much my understanding of moduli spaces. I am also very grateful to J. Mather for his patience in listening to a number of sometimes very confused and tentative presentations of these results and for his help in clarifying my ideas with numerous questions and suggestions. This research was supported by NSF grant DMS-9704791. 
9. The deviation of ergodic averages and open questions

9.1. The $L^{2}$ mean deviation and the cohomological equation

9.2. Sobolev estimates for the (first) return orbits

9.3. Special sequences of 'close' return times

9.4. The main theorem on the deviation of ergodic averages

9.5. The deviation for area-preserving vector fields and open questions

\section{Introduction}

Quantities of physical interest in a mechanical system are often described as time averages along the trajectories of the system. Since such averages are in general difficult to compute, a fundamental idea which goes back to Boltzmann is to replace them with averages over the phase space. In the mathematical formulation, a flow $\left(M, \Phi_{X}^{\tau}, \mu\right)$ on a manifold $M$, generated by a smooth vector field $X$, is ergodic if the time averages of any integrable function converge with probability one to the average with respect to the invariant probability measure $\mu$ as 'time' goes to infinity. It is therefore a relevant problem to establish quantitative estimates on the speed of convergence and on the asymptotic behaviour of time averages of functions in ergodic systems. However, a basic result in ergodic theory $[50, \S 3.2 \mathrm{~B}$, Th. 2.3$]$ states that for a general integrable function the speed of convergence can be arbitrarily slow. It is then reasonable to restrict the class of functions under consideration to functions with a certain degree of smoothness.

For many systems with strongly chaotic, hyperbolic behaviour the Central Limit Theorem (CLT) is known to hold. Examples include smooth uniformly hyperbolic systems, like geodesic flows on compact manifolds of negative curvature (Sinai, Ratner), discontinuous uniformly hyperbolic systems, like billiards with convex scatterers (Sinai's billiards) and non-uniformly hyperbolic systems, like Bunimovich's billiards (the reader can consult the survey by M. Denker [10] or the more recent papers by L.-S. Young [75], [76]). The CLT states that, for any Hölder continuous function $f$ on $M$,

$$
\frac{1}{\sqrt{\mathcal{T}}}\left\{\int_{0}^{\mathcal{T}} f\left(\Phi_{X}^{\tau}(p)\right) d \tau-\mathcal{T} \int_{M} f d \mu\right\} \rightarrow \mathcal{N}\left(0, \sigma_{f}\right)
$$

in the sense of probability distributions, where $\mathcal{N}\left(0, \sigma_{f}\right)$ is the normal (Gaussian) distribution with zero mean and variance $\sigma_{f}^{2}$. A related result, also known for some examples of hyperbolic systems [10], is the so-called Law of Iterated Logarithms (LIL). The LIL provides the following almost everywhere upper bound on the deviation of ergodic averages:

$$
\int_{0}^{\mathcal{T}} f\left(\Phi_{X}^{\tau}(p)\right) d \tau-\mathcal{T} \int_{M} f d \mu=\mathcal{O}(\sqrt{\mathcal{T} \log \log \mathcal{T}})
$$


If CLT and LIL hold, then the stochastic process $\left\{f \circ \Phi_{X}^{\tau}\right\}_{\tau \in \mathbb{R}}$ on the probability space $(M, \mu)$ behaves to some extent as if it were truely random (i.e. such as outcomes from flipping a coin or the Brownian motion).

In the non-hyperbolic case less is known. If the system is uniquely ergodic, the convergence of ergodic averages is uniform, with respect to $p \in M$, for all continuous functions. It is therefore natural to ask for pointwise estimates on the deviation of ergodic averages. A fundamental and well understood example, for its relevance in Hamiltonian mechanics, is given by regular quasi-periodic motions on tori, which have zero topological entropy, are never mixing but generically are uniquely ergodic. In particular, for a generic smooth aperiodic conservative flow on the 2-dimensional torus $T^{2}$, the Denjoy-Koksma inequality [25, Chap. VI, §3] and the metric (measure) theory of continued fractions [36, Chap. III, Ths. $30 \& 31$ ] imply that, if $f$ is a function of bounded variation, then for all $p \in T^{2}$ and any $\alpha>0$,

$$
\left|\int_{0}^{\mathcal{T}} f\left(\Phi_{X}^{\tau}(p)\right) d \tau-\mathcal{T} \int_{M} f d \mu\right| \leq C_{\alpha} \operatorname{Var}(f) \log \mathcal{T}(\log \log \mathcal{T})^{1+\alpha}
$$

In addition, if $f$ is sufficiently smooth, a Fourier series argument shows that

$$
\left|\int_{0}^{\mathcal{T}} f\left(\Phi_{X}^{\tau}(p)\right) d \tau-\mathcal{T} \int_{M} f d \mu\right| \leq C_{f}
$$

as a consequence of the existence of smooth solutions of the cohomological equation $X u=f-\int_{M} f d \mu$. Conservative aperiodic flows on tori are examples of elliptic dynamics, characterized by very slow or no divergence of nearby orbits. Hyperbolic systems display exponential divergence of orbits, which is the fundamental source of their strongly chaotic properties.

The above results leave open all intermediate cases. A lemma proved by M. Ratner [51, Lemma 3.1], based on the Chebychev inequality, states that polynomial decay of correlations implies polynomial speed of convergence of ergodic averages. According to Ratner, if a function $f$ has polynomial decay of correlation with exponent $0<\alpha_{f}<1$, then for any $\varepsilon>0$ there are $P:=P_{\varepsilon} \subset M$ with $\mu(P) \geq 1-\varepsilon$ and $\mathcal{T}_{P}>0$ such that, if $p \in P$,

$$
\left|\int_{0}^{\mathcal{T}} f\left(\Phi_{X}^{\tau}(p)\right) d \tau-\mathcal{T} \int_{M} f d \mu\right| \leq \mathcal{T}^{1-\alpha^{\prime}}
$$

for all $\mathcal{T} \geq \mathcal{T}_{P}$, where $\alpha^{\prime}:=\alpha_{f}^{\prime}=\alpha_{f} / 8$. Ratner's result was proved with the horocycle flow on a compact surface of constant negative curvature in mind and for which the required polynomial decay of correlations was known [52]. In the horocycle case Ratner's result was improved upon by M. Burger. He proved [7, Th. $2(\mathrm{C})]$ that, if $M$ is the unit cotangent bundle of a compact Riemannian surface $R$ of constant negative curvature, then (0.3) holds uniformly for all $p \in M$ with exponent $0<\alpha^{\prime}<1$ determined by the smallest non-zero 
eigenvalue of the Laplace-Beltrami operator on $R$. The results of Ratner [52] and Burger [7] are based on the Fourier analysis for the Lie group PSL $(2, \mathbb{R})$. The horocycle flow, which is uniquely ergodic and mixing, is an example of parabolic dynamics, characterized by polynomial divergence of nearby orbits.

In this paper we establish results on the asymptotic behaviour of ergodic averages for a class of conservative flows with isolated canonical saddle singularities on higher genus surfaces. Such flows were originally introduced and studied mainly by the Russian school as a simple model of the dynamics of Hamiltonian systems in the case of a 'multivalued' Hamiltonian, as in the presence of an electromagnetic field $[48, \S 6]$, or other first integral, as in the case of billiards in rational polygons and related systems [77]. L.-S. Young [74] proved that all continuous flows on surfaces have zero topological entropy. A.V. Kocergin $[37, \S 5$, Th. 3] established the mixing property for ergodic flows in case all singularities are (degenerate) mixing saddle points [37, Def. 5.3]. To the author's best knowledge, no satisfactory estimates for the decay of correlations are known and mixing is an open question in case one of the saddles is of Morse type (a recent result of B. Fayad [16, I, Th. 3.1] for flows on the torus suggests that flows with mixing saddle points should have polynomial decay of correlations). In contrast to the asymptotic behaviour of ergodic averages, which essentially depends only on the orbit foliation, the mixing property for flows with singularities is extremely sensitive to time changes. In fact, if the return time function (to a transverse interval) is of bounded variation, then the flow is not mixing [30]. In this case the flow cannot however be continuous. The (unique) ergodicity on the complement of the singular set for generic flows, known in the related case of interval exchange transformations as the Keane conjecture, was finally proved by H. Masur [43] and W. Veech [65].

A. Zorich, motivated by a question of S. P. Novikov $[48, \S 6]$ on the semiclassical trajectories of an electron on the Fermi surface of a metal $[82, \S \S 1.3$ $\&$ 1.4], has discovered in numerical experiments a striking non-standard behaviour of ergodic averages. In his papers [78]-[82] and his joint paper with M. Kontsevich [38] he has proved several partial results and formulated conjectures that draw a fairly complete picture of the new phenomenon. In [18] the author has proved the existence of distributional obstructions, not given by measures, to solving the cohomological equation $X u=f$. As a consequence, the estimate $\left(0.2^{\prime}\right)$ can be proved only for a finite codimensional subspace of the space of smooth functions (described by the vanishing of a finite number of invariant distributions), in agreement with Zorich's conjectures. This paper essentially completes Zorich's program on the deviation of ergodic averages. The approach followed is Zorich's and, especially, Kontsevich-Zorich's [38], except for the relevant role played by invariant distributions and by the methods developed in [18]. The results obtained below, together with those of [18], show 
that the dynamics of generic conservative flows on higher genus surfaces differs significantly from the genus one case. It appears in fact to be closer to the behaviour expected in the parabolic case, represented by the horocycle flow. This phenomenon can heuristically be explained by the divergence of nearby orbits produced by saddle-like singularities that are unavoidable in the higher genus case [33, Chap. 8, §4].

Let $M$ be a compact orientable surface of genus $g \geq 2$, let $(\Sigma, \imath)$ be a divisor on $M$, in the sense that $\Sigma:=\left\{p_{1}, \ldots, p_{\sigma}\right\}$ is a finite subset of $M$ and $\imath:=\left(\imath_{1}, \ldots, \imath_{\sigma}\right) \in \mathbb{Z}^{\sigma}$, with the properties that $\imath_{k}<0$ for all $k \in\{1, \ldots, \sigma\}$ and $\sum_{k} \imath_{k}=2-2 g$. Let $\mathcal{F}^{\imath}(M, \Sigma)$ be the space of orientable measured foliations, in the sense of W.Thurston [62], with (possibly degenerate) saddle-like singularities of canonical type at $\Sigma$, of index $\imath_{k}$ at $p_{k}$ for each $k \in\{1, \ldots, \sigma\}$, and no regular leaves homologous to zero. Let $\omega$ be any smooth non-negative 2-form on $M$, positive on $M \backslash \Sigma$, allowed to have zeroes at $\Sigma$. Let $\mathcal{E}_{\omega}^{\imath}(M, \Sigma)$ be the space of all smooth $\omega$-preserving vector fields on $M \backslash \Sigma$ such that the form $\eta_{X}:=\imath_{X} \omega$ is smooth (and closed) on $M$ and the orbit foliation $\mathcal{F}_{X}:=\left\{\eta_{X}=0\right\} \in \mathcal{F}^{\imath}(M, \Sigma)$. The main goal of the paper is to prove the following result, conjectured by M. Kontsevich and A. Zorich [38] on the basis of numerical experiments:

Theorem 0.1 (Theorem 9.6). For 'almost all' $X \in \mathcal{E}_{\omega}^{\imath}(M, \Sigma)$, the flow $\Phi_{X}^{\tau}$ has a deviation spectrum in the following sense. There exists a finite set of exponents

$$
\lambda_{1}^{\prime}(X)=1>\lambda_{2}^{\prime}(X)>\cdots>\lambda_{s}^{\prime}(X)>0
$$

and a splitting of the space $\mathcal{I}_{X}^{1}(M)$ of $X$-invariant distributions of order 1 (i.e. solutions $\mathcal{D} \in H^{-1}(M)$ of the equation $X \mathcal{D}=0$ )

$$
\mathcal{I}_{X}^{1}(M)=\mathcal{I}_{X}^{1}\left(\lambda_{1}^{\prime}\right) \oplus \mathcal{I}_{X}^{1}\left(\lambda_{2}^{\prime}\right) \oplus \cdots \oplus \mathcal{I}_{X}^{1}\left(\lambda_{s}^{\prime}\right)
$$

such that the following holds. Let $f \in H_{0}^{1}(M \backslash \Sigma)$ be a weakly differentiable function with $L^{2}$ partial derivatives, supported in $M \backslash \Sigma$, such that

$$
\mathcal{D}^{X}(f)=0, \quad \text { for all } \mathcal{D}^{X} \in \mathcal{I}_{X}\left(\lambda_{1}^{\prime}\right) \oplus \cdots \oplus \mathcal{I}_{X}\left(\lambda_{i}^{\prime}\right) .
$$

If $i<s$ in (0.5), then for all $p \in M$ with regular forward trajectory,

$$
\limsup _{\mathcal{T} \rightarrow+\infty} \frac{\log \left|\int_{0}^{\mathcal{T}} f\left(\Phi_{X}(p, \tau)\right) d \tau\right|}{\log \mathcal{T}} \leq \lambda_{i+1}^{\prime}(X)
$$

and there exists $\mathcal{D}_{i+1}^{X} \in \mathcal{I}_{X}\left(\lambda_{i+1}^{\prime}\right) \backslash\{0\}$ such that, if $\mathcal{D}_{i+1}^{X}(f) \neq 0$, then equality holds in (0.6) for almost all $p \in M$.

If $i=s$ in (0.5), then, for all $p \in M$ with regular forward trajectory,

$$
\limsup _{\mathcal{T} \rightarrow+\infty} \frac{\log \left|\int_{0}^{\mathcal{T}} f\left(\Phi_{X}(p, \tau)\right) d \tau\right|}{\log \mathcal{T}}=0 .
$$


The multiplicities $m_{i}(X):=\operatorname{dim} \mathcal{I}_{X}\left(\lambda_{i}^{\prime}\right)$ satisfy the conditions $\sum_{i} m_{i}(X)=g$ and $m_{1}(X)=1$, since $\mathcal{I}_{X}\left(\lambda_{1}^{\prime}\right)=\mathbb{R} \cdot \omega$. The deviation spectrum with multiplicities $\left\{\left(\lambda_{i}^{\prime}(X), m_{i}(X)\right) \mid i=1, \ldots, s\right\}$ is locally constant on $\mathcal{E}_{\omega}^{\imath}(M, \Sigma)$.

The measure class on $\mathcal{E}_{\omega}^{\imath}(M, \Sigma)$ is the (standard) one obtained by considering the Lebesgue measure on the A. Katok's fundamental classes of areapreserving vector fields. The fundamental class of a vector field $X \in \mathcal{E}_{\omega}(M, \Sigma)$ is, following [29], the cohomology class of the closed 1-form $\eta_{X}:=\imath_{X} \omega$ in $H^{1}(M, \Sigma ; \mathbb{R})$. The pull-back of the Lebesque measure class on the cohomology vector space under the map $\mathcal{E}_{\omega}^{\imath}(M, \Sigma) \rightarrow H^{1}(M, \Sigma ; \mathbb{R})$, given by $X \rightarrow\left[\eta_{X}\right]$, yields the measure class considered in the statement of Theorem 0.1. The symbols $H^{1}(M), H^{-1}(M)$ denote the standard $L^{2}$ Sobolev spaces on the compact manifold $M$ [1]. The space $H^{1}(M)$ is defined as the space of $L^{2}$ weakly differentiable functions with $L^{2}$ partial derivatives and the space $H^{-1}(M)$ as the dual of the Banach space $H^{1}(M)$. The space $H_{0}^{1}(M \backslash \Sigma) \subset H^{1}(M)$ is the subspace of functions with compact support in $M \backslash \Sigma$.

In the case $i=0$, the statement of Theorem 0.1 follows immediately from the (unique) ergodicity of 'almost all' vector fields (the Keane Conjecture, proved by [43], [65] and later on by several other authors by different methods) and from Birkhoff's ergodic theorem. In fact, the continuous embedding $H^{1}(M) \subset L^{1}(M, \omega)$ holds by the Hölder inequality. In case the function $f$ has zero average, by the theory of sign changes of ergodic integrals [50, Chap. $3(\mathrm{C})$ ], the lower limit of the quantity in (0.6) is $-\infty$ and the limit does not exist for almost all $p \in M$. In the related case of interval exchange transformations, A. Zorich [80], [82] proved a statement similar to Theorem 0.1 for linear combinations of characteristic functions of the sub-intervals. He conjectured that all the exponents in (0.4) are non-zero and that the spectrum is simple, in the sense that all the multiplicities $m_{1}=\cdots=m_{s}=1$ (hence $s=g$ ), as suggested by the numerical evidence and the results he had presented in [78], [79], [80]. We were unable to prove the part of the conjecture concerning the simplicity of the spectrum, except in the case $g=2$. In [38] M. Kontsevich and A. Zorich conjectured that, in the case of area-preserving flows on surfaces, Zorich's theorem could be generalized to smooth functions. The role played by invariant distributions was not part of the Kontsevich-Zorich picture. The author regards it as his most important contribution to the subject.

The proof of Theorem 0.1 is based on several results, concerning the Teichmüller geodesic flow, which, we believe, are of independent interest. Following the conjectural approach outlined in [38], we have proved the non-uniform hyperbolicity of a 'renormalization' cocycle, introduced in [38], and earlier in a different form in [79], [80]. The Kontsevich-Zorich cocycle is a flow on a symplectic vector bundle over the moduli space $\mathcal{M}_{g}$ of holomorphic quadratic 
differentials on Riemann surfaces of genus $g \geq 2$. The fiber of the vector bundle at a quadratic differential $q$ is the cohomology vector space with real coefficients $H^{1}\left(M_{q}, \mathbb{R}\right)$ of the Riemann surface $M_{q}$ carrying $q$. The cocycle is defined as a lift of the Teichmüller geodesic flow, obtained by parallel transport of cohomology classes with respect the canonical flat (Gauss-Manin) connection of the bundle. Since the Kontsevich-Zorich cocycle is defined on a symplectic vector bundle (of dimension $2 g$ ), it has a symmetric Lyapunov spectrum:

$$
\lambda_{1}=1 \geq \lambda_{2} \geq \cdots \geq \lambda_{g} \geq 0 \geq-\lambda_{g} \geq \cdots-\lambda_{2} \geq-\lambda_{1}=-1 .
$$

The numbers $\lambda_{1}^{\prime}(X)>\cdots>\lambda_{s}^{\prime}(X)>0$ appearing in the statement of Theorem 0.1 are the distinct positive Lyapunov exponents of the Kontsevich-Zorich cocycle with respect to the canonical absolutely continuous invariant measure of the Teichmüller flow on the appropriate connected component of a stratum of the moduli space determined by $X \in \mathcal{E}_{\omega}^{\imath}(M, \Sigma)$. We prove the following result:

Theorem 0.2 (Corollary $2.2 \&$ Th. 8.5). (i) The first Lyapunov exponent $\lambda_{1}^{\mu}=1$ of the Kontsevich-Zorich cocycle, with respect to any ergodic probability measure $\mu$ of the Teichmüller flow on the stratum of squares of holomorphic differentials, is simple. In fact, the second Lyapunov exponent $\lambda_{2}^{\mu}<1$.

(ii) The Kontsevich-Zorich cocycle is non-uniformly hyperbolic with respect to the canonical absolutely continuous invariant probability measure of the Teichmüller flow, in the sense that, for (Lebesgue) almost all quadratic differentials on each connected component of a stratum of squares,

$$
\lambda_{1}=1>\lambda_{2} \geq \cdots \geq \lambda_{g}>0 .
$$

The Lyapunov spectrum of the Kontsevich-Zorich cocycle contains all the non-trivial information on the Lyapunov spectrum of the Teichmüller geodesic flow, which was proved by W. Veech [66] to be non-uniformly hyperbolic on each connected component of a stratum of quadratic differentials, with respect to the canonical absolutely continuous invariant probability measure. In fact, the explicit formulas $\left(1.2^{\prime}\right)$ hold and Theorem 0.2 is therefore equivalent to the following:

Theorem $0.2^{\prime}$. (i) All probability invariant measures of the Teichmüller geodesic flow, in particular all periodic trajectories, on any stratum of squares, are non-uniformly hyperbolic, in the sense that the Lyapunov exponent 0 (corresponding to the direction of the flow) is simple.

(ii) The multiplicity of the Lyapunov exponent 1, on every connected component of a stratum of squares having $\sigma \in\{1, \ldots, 2 g-2\}$ distinct zeroes, is almost everywhere exactly equal to $\sigma-1$, with respect to the canonical absolutely continuous invariant probability measure. 
The proof of Theorem 0.2 (and of Theorem $0.2^{\prime}$ ), carried out in Sections 2-5 and Section 8, is based on explicit equations for the Kontsevich-Zorich cocycle in appropriate coordinates, established in Section 2. By applying such equations, we are able to obtain a remarkably simple proof of Theorem 0.2 (i) (Corollary 2.2) and to compute formulas for the hyperbolic Laplacian of the logarithm of the volume on any isotropic $k$-plane in $H^{1}(M, \mathbb{R})$ over Teichmüller disks (in $\S 3$ and $\S 5$ ). In the particular case $k=g$, we obtain a new version of the formula obtained in [38]. The argument is thus reduced to a geometric problem concerning the determinant of the directional derivatives of the period matrix, which is solved in Section 4 by studying quadratic differentials near special boundary points of the moduli space. The complete proof of the nonuniform hyperbolicity of the Kontsevich-Zorich cocycle (Theorem 8.5) depends on additional information on the invariant sub-bundles (which could be in fact deduced, as remarked by A. Eskin, from [66]), studied in Section 6 and Section 8.

Any holomorphic quadratic differential $q$ on a Riemann surface $M_{q}$ can be identified with a pair $\left(\mathcal{F}_{q}, \mathcal{F}_{-q}\right)$ of transverse measured foliations (in the sense of [62]), the horizontal foliation $\mathcal{F}_{q}:=\left\{\Im\left(q^{1 / 2}\right)=0\right\}$, the vertical foliation $\mathcal{F}_{-q}:=\left\{\Re\left(q^{1 / 2}\right)=0\right\}$. Let $q$ be the square of a holomorphic (abelian) differential which is a regular point (in the sense of the Oseledec's theorem [49], $\left[32\right.$, Th. S.2.9]) of the Kontsevich-Zorich cocycle. Let $E_{q}^{+}\left[E_{q}^{-}\right] \subset H^{1}\left(M_{q}, \mathbb{R}\right)$ the unstable [the stable] subspace at $q$ of the Kontsevich-Zorich cocycle. Such spaces have a dynamical interpretation, discovered by A. Zorich [78], in terms of the foliations $\mathcal{F}_{ \pm q}$. Let $\widehat{\gamma}_{ \pm q}^{\mathcal{T}}$ be the closed curve on $M$ obtained as the union of a leaf of length $\mathcal{T}>0$ of the foliation $\mathcal{F}_{ \pm q}$ and of a 'short' segment of length $\leq \operatorname{diam}\left(M_{q}\right)$. Then the distance of the homology class $\left[\widehat{\gamma}_{ \pm q}^{\mathcal{T}}\right] \in H_{1}(M, \mathbb{R})$ from the Poincaré dual of the subspace $E_{q}^{ \pm}$is (uniformly) bounded for all $\mathcal{T}>0$. The cycles contained in the Poincaré dual of the subspace $E_{q}^{ \pm}$have been called $[47, \S 7.9 .3]$ the Zorich cycles of the measured foliation $\mathcal{F}_{ \pm q}$. The Schwartzman [57] asymptotic cycle of the foliation $\mathcal{F}_{ \pm q}$ is a particular Zorich cycle. It follows from Theorem 0.2(ii) that the dimension of the space of Zorich cycles is 'generically' equal to the genus $g \geq 2$.

We have proved that all cohomology classes in the $E_{q}^{ \pm}$, i.e. all the Poincaré duals of Zorich cycles of $\mathcal{F}_{ \pm q}$, can be represented by (closed) currents with special properties. Let $\Sigma_{q} \subset M$ be the finite set of the zeroes of the quadratic differential $q$. A current of dimension (and degree) equal to 1 on the 2-dimensional manifold $M \backslash \Sigma_{q}$ is a continuous linear functional on the space of smooth 1-forms with compact support [11], [56, Chap. IX]. A basic current for the measured foliation $\mathcal{F}_{ \pm q}$ is defined as a distributional generalization of the notion of basic form, well known in the geometric theory of foliations since the work of B. L. Reinhart [53], [54] (see also [2, §1.5 \& §7], [63, Chap. 4]). A current $C$ of dimension (and degree) equal to 1 is basic for the measured foliation $\left.\mathcal{F}_{ \pm q}\right|_{M \backslash \Sigma_{q}}$ 
if, for all vector fields $X$ tangent to $\mathcal{F}_{ \pm q}$ with compact support in $M \backslash \Sigma_{q}$,

$$
\imath_{X} C=\mathcal{L}_{X} C=0 .
$$

The operation of contraction $\imath_{X}$ and Lie derivative $\mathcal{L}_{X}$ are extended to currents in the standard distributional sense [56, Chap. IX, §3]. If a current $C$ on $M \backslash \Sigma_{q}$ (which is 2-dimensional) is basic then it is closed, hence it represents, by the generalized de Rham theorem [11, Th. 12], [56, Chap. IX, §3, Th. I], a cohomology class in $H^{1}\left(M \backslash \Sigma_{q}, \mathbb{R}\right)$. Let $X^{ \pm} \in \mathcal{E}_{\omega}^{\imath}\left(M, \Sigma_{q}\right)$ be vector fields respectively tangent to the leaves of $\mathcal{F}_{q}^{ \pm}$. There is a one-to-one correspondence between the space of $X^{ \pm}$-invariant distributions on $M \backslash \Sigma_{q}$ and the space of basic currents for $\left.\mathcal{F}_{ \pm q}\right|_{M \backslash \Sigma_{q}}$. In fact, if $\mathcal{D}^{ \pm}$is any $X^{ \pm}$-invariant distribution, then its contraction $\imath_{X^{ \pm}} \mathcal{D}^{ \pm}$is a current of dimension (and degree) equal to 1 which is basic for $\mathcal{F}_{ \pm q}$. The $X^{ \pm}$-invariant measures are a particular case of $X^{ \pm}$-invariant distributions and the related basic currents where implicitly considered by A. Katok [29].

The space of currents on $M \backslash \Sigma_{q}$ is filtered by a sequence of weighted Sobolev spaces $\mathcal{H}_{q}^{-s}(M), s \geq 0$. A basic current for $\mathcal{F}_{ \pm q}$, of finite order $s \geq 0$, is a current $C \in \mathcal{H}_{q}^{-s}(M)$ of dimension (and degree) equal to 1 such that the identities (0.8) hold for all vector fields $X$ tangent to $\mathcal{F}_{ \pm q}$ for which the operators $\imath_{X}: \mathcal{H}_{q}^{-s}(M) \rightarrow \mathcal{H}_{q}^{-s}(M)$ and $\mathcal{L}_{X}: \mathcal{H}_{q}^{-s}(M) \rightarrow \mathcal{H}_{q}^{-s-1}(M)$ are well defined. Basic currents of order 0 correspond to absolutely continuous invariant measures. It can be proved (see Lemma 6.2) that the cohomology class of a basic current for $\mathcal{F}_{ \pm q}$, of order $s \geq 0$, belongs to the cohomology vector space $H^{1}(M, \mathbb{R}) \subset H^{1}\left(M \backslash \Sigma_{q}, \mathbb{R}\right)$. The following result describes the invariant subbundles of the Kontsevich-Zorich cocycle in terms of basic currents:

TheOREM 0.3 (Theorem 8.3). For almost all quadratic differential $q$ in every stratum of squares of holomorphic differentials, the invariant unstable [stable] subspace at $q$ of the Kontsevich-Zorich cocycle is the subspace $E_{q}^{+}\left[E_{q}^{-}\right] \subset H^{1}\left(M_{q}, \mathbb{R}\right)$ given by the cohomology classes of the basic currents for $\mathcal{F}_{q}\left[\mathcal{F}_{-q}\right]$ which are of order 1 .

Theorem 0.3 is in fact valid with respect to any $\mathrm{SL}(2, \mathbb{R})$ invariant probability measure on the moduli space and it requires no information on the dimension of the stable or unstable sub-bundle. The proof is based on a Cheegertype estimate of the first non-trivial eigenvalue of the Dirichlet form of the flat metric induced by a quadratic differential, proved in Section 6.3, and on the logarithmic law for geodesics in the moduli space, proved in [44].

Theorem 0.2 and Theorem 0.3 imply that the dimension of the space of basic current of order 1 for a 'generic' measured foliation on a compact orientable surface $M$ is equal to the genus $g \geq 2$ of $M$. It is interesting to compare such a result with the results we have proved in Section 7 on the space of all basic currents of finite order. 
Theorem 0.4 (Theorems $7.1 \&$ 7.7). Let $\eta_{\mathcal{F}}$ denote the smooth closed 1-form such that $\mathcal{F}:=\left\{\eta_{\mathcal{F}}=0\right\}$.

(i) There exists $r>1$ such that, for almost all orientable measured foliations $\mathcal{F} \in \mathcal{F}_{\omega}^{\imath}(M, \Sigma)$, the space $H_{\mathcal{F}}^{1, s}(M, \mathbb{R})$ of cohomology classes of $\mathcal{F}$-basic currents of any order $s \geq r$ (called the $H^{-s}$ basic cohomology of $\mathcal{F}$ ) has codimension 1 in the cohomology $H^{1}(M, \mathbb{R})$. In fact,

$$
H_{\mathcal{F}}^{1, s}(M, \mathbb{R}):=\left\{c \in H^{1}(M, \mathbb{R}) \mid c \wedge\left[\eta_{\mathcal{F}}\right]=0\right\} .
$$

(ii) Let $X$ be any smooth vector field on $M \backslash \Sigma$ such that $\imath_{X} \eta_{\mathcal{F}} \equiv 1$. Let $C$ be an $\mathcal{F}$-basic current of order $s \geq 0$ such that the cohomology class $[C]=0 \in H^{1}(M, \mathbb{R})$. Then there exists an $\mathcal{F}$-basic current $\widehat{C}$ of order $s-1$ such that $C=d \imath_{X} \widehat{C}$. (The restriction of the operator $d \circ \imath_{X}$ to $\mathcal{F}$-basic currents does not depend on the choice of the vector field $X$ ).

By Theorem 0.4(i), the regularity restriction on basic current in the statement of Theorem 0.3 is crucial. Theorem 0.4(ii) implies that there are only a finite number of 'primitive' basic currents for any measured foliation. However, since $2 g-1>g$, by Theorem $0.4(\mathrm{i})$, not all of them have a dynamical significance, according to Theorem 0.1 or Theorem 0.3 . The question whether all basic currents (with non-vanishing cohomology class) have a dynamical interpretation is left unanswered (see Question 9.10). The proof of Theorem 0.4 is based on the results of [18].

In order to prove Theorem 0.1, we introduce a cocycle $G_{t}^{c}$ over the Teichm̈uller flow $G_{t}$, defined on the infinite dimensional bundle $Z^{-1}(M)$ over the moduli space with fiber given by the space of all closed currents of order 1 . The projection of the cocycle $G_{t}^{c}$ under the map $Z_{q}^{-1}(M) \rightarrow H^{1}\left(M_{q}, \mathbb{R}\right)$, given by the generalized de Rham theorem, coincides with the Kontsevich-Zorich cocycle. There is no general Oseledec's theorem for infinite dimensional (Hilbert) bundles and the available ones (see the survey [55]) do no seem to apply to the case at hand. However, we derive from Theorem 0.2 and Theorem 0.3 the following Oseledec-type splitting:

TheOREM 0.5 (Theorem 8.7). There is a $G_{t}^{c}$-invariant splitting

$$
Z^{-1}(M) \equiv \mathcal{B}_{+}^{1}(M) \oplus \mathcal{B}_{-}^{1}(M) \oplus \mathcal{E}^{-1}(M) .
$$

(i) $\mathcal{B}_{ \pm}^{1}(M)$ is the bundle of $\mathcal{F}_{ \pm q}$-basic currents of order 1 over the moduli space of squares of holomorphic differentials.

(ii) The restriction of the cocycle $G_{t}^{c}$ to the bundle $\mathcal{B}_{+}^{1}(M) \oplus \mathcal{B}_{-}^{1}(M)$ is measurably isomorphic to the Kontsevich-Zorich cocycle on the cohomology bundle.

(iii) The Lyapunov spectrum of the cocycle $G_{t}^{c}$ on $\mathcal{B}_{ \pm}^{1}(M)$ consists of the set of Lyapunov exponents $\pm\left\{\lambda_{1}, \ldots, \lambda_{g}\right\}$ given by $\left(0.7^{\prime}\right)$. 
(iv) The fiber of the bundle $\mathcal{E}^{-1}(M)$ at $q$ consists of all exact currents of order 1 . The Lyapunov spectrum of the cocycle $G_{t}^{c}$ on $\mathcal{E}^{-1}(M)$ is reduced to the single Lyapunov exponent 0.

The strategy of the proof of Theorem 0.1 is then clarified by the remark that the return orbits of a flow $\Phi_{X}^{\tau}, X \in \mathcal{E}_{\omega}^{\imath}(M, \Sigma)$, closed by a (short) transverse segment, are closed currents of order 1, by the Sobolev embedding theorem [1, Th. 5.4]. Hence, their evolution under the action of the cocycle $G_{t}^{c}$ can be studied by applying Theorem 0.5. Since $G_{t}^{c}$ is a cocycle over the Teichmüller flow, which plays the role of a renormalization dynamics for the flows of the vector fields $X \in \mathcal{E}_{\omega}^{\imath}(M, \Sigma)$, Theorem 0.1 can be derived from Theorem 0.5 by standard techniques. The $X$-invariant distributions appearing in the statement of Theorem 0.1 are in one-to-one correspondence with the basic currents of order 1 for the orbit foliation $\mathcal{F}_{X}$ given by Theorem 0.2(ii) and Theorem 0.3.

\section{The Kontsevich-Zorich cocycle}

Let $T_{g}$ be the Teichmüller space of all marked closed Riemann surfaces of genus $g \geq 2$. It can be viewed as the quotient of the space of all complex structures on a compact orientable topological surface of genus $g$, with respect to the equivalence relation induced by the natural action of the group $\operatorname{Diff}_{0}^{+}(M)$ of orientation-preserving diffeomorphisms homotopic to the identity. For a general introduction to Teichmüller theory and references to the relevant literature we refer to the survey article by L. Bers [6]. In particular, $T_{g}$ has a natural complex structure (Alhfors-Bers), isomorphic to that of a bounded domain of holomorphy of the Euclidean space $\mathbb{C}^{3 g-3}$, and it can be equipped with a natural Finsler metric (the Teichmüller metric). The quotient of the bundle of non-zero holomorphic quadratic differentials on marked closed Riemann surfaces of genus $g$, with respect to the natural action of $\operatorname{Diff}_{0}^{+}(M)$ by pull-back, is a complex manifold $Q_{g}$, which can be identified with the cotangent bundle of $T_{g}$ (minus the zero section). We will be concerned with subvarieties of $Q_{g}$ given by holomorphic quadratic differentials with a prescribed pattern of zeroes.

Let $\kappa=\left(k_{1}, \ldots, k_{\sigma}\right)$ satisfy the following properties: each $k_{i}$ is a positive even integer and $\sum k_{i}=4 g-4$. We define $Q_{\kappa}$ to be the subset of $Q_{g}$ consisting of quadratic differentials $q$ such that (1) $q$ is the square of a holomorphic (abelian) differential; $(2)$ the distinct zeroes of $q$ have orders $\left(k_{1}, \ldots, k_{\sigma}\right)$. The set $Q_{\kappa}$ is a complex analytic subvariety of $Q_{g}$, which is called a stratum of $Q_{g}$. Since $\sum k_{i}=4 g-4$, the number of strata in $Q_{g}$ is finite. The strata of $Q_{g}$ which correspond to non-orientable quadratic differentials will not be considered here. 
They can be studied by passing to suitable branched double covers over $M$. If $q \in Q_{\kappa}$, we denote by $M_{q}$ be the Riemann surface carrying $q$ and by $\Sigma_{q}$ the finite set of distinct zeroes of $q$.

Let $\Gamma_{g}:=\operatorname{Diff}^{+}(M) / \operatorname{Diff}_{0}^{+}(M)$ be the pure mapping class group of the compact orientable surface $M$ of genus $g \geq 2$. It is defined as the group of isotopy equivalence classes of orientation-preserving diffeomorphisms of $M$. The mapping class group acts by pull-back on $T_{g}, Q_{g}, Q_{\kappa}$. Such actions are properly discontinuous and given by biholomorphic isometries (with respect to the Teichmüller metric). The quotient spaces $R_{g}:=T_{g} / \Gamma_{g}, \mathcal{M}_{g}:=Q_{g} / \Gamma_{g}$ are, respectively, the moduli spaces of complex structures and of quadratic differentials on surfaces of genus $g$. Since there are Riemann surfaces with non-trivial (but finite) automorphism group, $R_{g}, Q_{g}$ are complex orbifolds (hence in particular complex analytic spaces) of dimension $3 g-3$, i.e. for each point $p \in R_{g}\left[p \in \mathcal{M}_{g}\right]$ there is a neighbourhood $\mathcal{U}_{p}$, a finite subgroup $G \subset \mathrm{GL}(3 g-3, \mathbb{C})[\mathrm{GL}(6 g-6, \mathbb{C})]$ and a neighbourhood $\tilde{\mathcal{U}}_{p}$ of the origin in $\mathbb{C}^{3 g-3}$ $\left[\mathbb{C}^{6 g-6}\right]$, such that $\mathcal{U}_{p} \equiv \tilde{\mathcal{U}}_{p} / G$. For each symbol $\kappa$, the quotient $\mathcal{M}_{\kappa}:=Q_{\kappa} / \Gamma_{g}$ is called a stratum of the moduli space $\mathcal{M}_{g}$. From their definition, it follows that strata are complex analytic subvarieties of $\mathcal{M}_{g}$. Moreover, each stratum $\mathcal{M}_{\kappa}$ can be equipped with the structures listed below [66], [68], [38]:

(1) a natural complex affine orbifold structure modeled on the vector space $H^{1}\left(M, \Sigma_{\kappa} ; \mathbb{C}\right)$, where $\Sigma_{\kappa}$ is a finite subset of $M$ with $\operatorname{card}\left(\Sigma_{\kappa}\right)=\sigma_{\kappa}$;

(2) an absolutely continuous measure $\mu_{\kappa}$;

(3) a locally quadratic non-holomorphic function $A: \mathcal{M}_{\kappa} \rightarrow \mathbb{R}^{+}$;

(4) a non-holomorphic action of the group $G L_{+}(2, \mathbb{R})$.

The structure (1) is given by the period map. There exists a locally defined continuous square root on $Q_{\kappa}$, such that if $q \in Q_{\kappa}, q^{1 / 2}$ is a holomorphic (abelian) differential with zeroes at $\Sigma_{q}$. The cohomology class of $q^{1 / 2}$ in $H^{1}\left(M_{q}, \Sigma_{q} ; \mathbb{C}\right)$ is defined by integration of $q^{1 / 2}$ along relative cycles in $\left(M_{q}, \Sigma_{q}\right)$. The period map gives a natural complex affine structure on $Q_{\kappa}$, modeled on the vector space $H^{1}\left(M, \Sigma_{\kappa} ; \mathbb{C}\right)$, where $\Sigma_{\kappa}$ is a finite set with $\operatorname{card}\left(\Sigma_{\kappa}\right)=\sigma$. In fact, the cohomology $H^{1}\left(M_{\tilde{q}}, \Sigma_{\tilde{q}} ; \mathbb{C}\right)$ can be canonically identified with $H^{1}\left(M_{q}, \Sigma_{q} ; \mathbb{C}\right)$, by parallel transport with respect to the so-called Gauss-Manin connection [23, §2], on a neighbourhood $\mathcal{U}_{q}$ of $q \in Q_{\kappa}$. The Gauss-Manin connection in this context is simply given by the property that parallel sections are the holomorphic sections of the holomorphic vector bundle over $Q_{\kappa}$ with fiber $H^{1}\left(M_{q}, \Sigma_{q} ; \mathbb{C}\right)$ which are locally constant. Hence the period map $\Pi_{q}$ is defined on $\mathcal{U}_{q}$ into $H^{1}\left(M_{q}, \Sigma_{q} ; \mathbb{C}\right)$ and is a (local) biholomorphism of $\mathcal{U}_{q}$ onto an open subset of $H^{1}\left(M_{q}, \Sigma_{q} ; \mathbb{C}\right)$. This result can be proved by studying holomorphic deformations of quadratic differentials in terms analogous to the Kodaira-Spencer theory of deformations of complex structures [27, Chap. IV, §2]. The complex affine structure on $Q_{\kappa}$, 
just described, induces, by quotient with respect to the action of the mapping class group $\Gamma_{g}$, a complex affine orbifold structure on the stratum $\mathcal{M}_{\kappa}$ in the moduli space.

The construction of the measure $\mu_{\kappa}$ in (2) is also based on the period map. In fact, the Lebesgue measure on $H^{1}\left(M_{q}, \Sigma_{q} ; \mathbb{C}\right)$, uniquely normalized by the condition that the volume of the $2 g-1+\sigma$ complex torus obtained as a quotient over the integer lattice $\mathbb{C} \otimes_{\mathbb{Z}} H^{1}\left(M_{q}, \Sigma_{q} ; \mathbb{Z}\right)$ is equal to 1 , gives a smooth measure on $Q_{\kappa}$, which projects by push-forward to a measure $\mu_{\kappa}$ on $\mathcal{M}_{\kappa}$.

The total area function $A: Q_{g} \rightarrow \mathbb{R}^{+}$is defined for any quadratic differential $q$ as the total area of the surface $M_{q}$ with respect to the area form $\omega_{q}$ of the metric $R_{q}$ induced by $q[58,5.3]$. The function $A$ is invariant under the action of $\Gamma_{g}$, hence it can be viewed as a function on $\mathcal{M}_{g}$.

Finally, the group $\mathrm{GL}_{+}(2, \mathbb{R})$ of $2 \times 2$ matrices with positive determinant acts on $Q_{\kappa}$ through the linear transformations on the pairs of real-valued 1-forms $\left(\Re\left(q^{1 / 2}\right), \Im\left(q^{1 / 2}\right)\right)$. In the affine coordinate system described by (1), it is the action of the group $\mathrm{GL}_{+}(2, \mathbb{R})$ on the vector space

$$
H^{1}\left(M_{q}, \Sigma_{q} ; \mathbb{C}\right) \equiv \mathbb{C} \otimes_{\mathbb{R}} H^{1}\left(M_{q}, \Sigma_{q} ; \mathbb{R}\right) \equiv \mathbb{R}^{2} \otimes_{\mathbb{R}} H^{1}\left(M_{q}, \Sigma_{q} ; \mathbb{R}\right)
$$

through the first factor in the tensor product. Such an action commutes with the action of the mapping class group $\Gamma_{g}$ and it induces therefore an action on $\mathcal{M}_{\kappa}$.

It follows immediately from the definitions that the subgroup $\operatorname{SL}(2, \mathbb{R})$ of $2 \times 2$ matrices with determinant equal to 1 preserves the measure $\mu_{\kappa}$ and the area function $A$. Consequently, on the hypersurface $Q_{\kappa}^{(1)}:=A^{-1}(1) \cap Q_{\kappa}$ we can define an induced smooth measure $\mu_{\kappa}^{(1)}:=\mu_{\kappa} / d A$ which is preserved by the action of the group $\operatorname{SL}(2, \mathbb{R})$. The action of the 1-parameter subgroup of diagonal matrices $G_{t}:=\operatorname{diag}\left(e^{t}, e^{-t}\right)$ gives a measure-preserving flow on $Q_{g}$, which preserves each stratum $Q_{\kappa}$ and each hypersurface $Q_{\kappa}^{(1)}$. Since the action of $\mathrm{GL}_{+}(2, \mathbb{R})$ commutes with the action of the mapping class group $\Gamma_{g}, G_{t}$ induces a measure preserving flow on the moduli space $\mathcal{M}_{g}$, which preserves each stratum $\mathcal{M}_{\kappa}$ and each hypersurface $\mathcal{M}_{g}^{(1)}:=A^{-1}(1) \cap \mathcal{M}_{g}$, $\mathcal{M}_{\kappa}^{(1)}:=A^{-1}(1) \cap \mathcal{M}_{\kappa}$. Such a flow is known as the Teichmüller geodesic flow. As discovered by W.Veech [68] the strata are not always connected. A complete description of all connected components of all strata has been given conjecturally in [38] and has been recently proved in [39] for all the strata of squares. In this paper we will not rely on such a description. By a theorem of H. Masur [43] and W. Veech [66] the Teichmüller flow is ergodic in the following sense: 
Theorem 1.1 ([43], [66]). The total volume of the measure $\mu_{\kappa}^{(1)}$ on $\mathcal{M}_{\kappa}^{(1)}$ is finite and the Teichmüller geodesic flow $G_{t}=\operatorname{diag}\left(e^{t}, e^{-t}\right)$ is ergodic on each connected component of $\mathcal{M}_{\kappa}^{(1)}$ with respect to the invariant measure $\mu_{\kappa}^{(1)}$.

In [38] M. Kontsevich and A. Zorich introduced a natural cocycle over the Teichmüller geodesic flow defined on a bundle over $\mathcal{M}_{\kappa}^{(1)}$ with fiber $H^{1}\left(M_{q}, \mathbb{R}\right)$. Such a cocycle plays the role of a renormalization group dynamics for flows along measured foliations and for interval exchange transformations, as it will appear from the content of Section 9 . Let $H_{g}^{1}(M, \mathbb{C})$ be the holomorphic vector bundle with fiber $H^{1}\left(M_{q}, \mathbb{C}\right)$ over the space $Q_{g}^{(1)}$ of holomorphic quadratic with unit total area. Since $Q_{g}^{(1)}$ is simply connected, the vector bundle $H_{g}^{1}(M, \mathbb{C})$ can be trivialized by parallel transport with respect to the Gauss-Manin connection, i.e. $H_{g}^{1}(M, \mathbb{C}) \equiv Q_{g}^{(1)} \times H^{1}(M, \mathbb{C})$ (Riemann surfaces in the Teichmüller space are marked). The Teichmüller geodesic flow $G_{t}$ on $Q_{g}^{(1)}$ lifts trivially to the product bundle by the identity action on the second factor. Hence, it lifts to a flow on $H_{g}^{1}(M, \mathbb{C}$ ) (in other terms, cohomology classes are moved by parallel transport along the Teichmüller flow). Such a flow induces, by quotient with respect to the mapping class group $\Gamma_{g}$, a flow on the complex affine (orbifold) vector bundle $\mathcal{H}_{g}^{1}(M, \mathbb{C}):=H_{g}^{1}(M, \mathbb{C}) / \Gamma_{g}$ which preserves real and imaginary parts of the bundle. The restriction of the flow to the real sub-bundle $\mathcal{H}_{g}^{1}(M, \mathbb{R}):=H_{g}^{1}(M, \mathbb{R}) / \Gamma_{g}$, introduced in [38], will be called the Kontsevich-Zorich cocycle and denoted by $G_{t}^{K Z}$. Such a cocycle leaves invariant each stratum $\mathcal{H}_{\kappa}^{1}(M, \mathbb{R})$, defined as the restriction to $\mathcal{M}_{\kappa}^{(1)}$ of the (orbifold) vector bundle $\mathcal{H}_{g}^{1}(M, \mathbb{R})$.

Since $H^{1}(M, \mathbb{R})$ has a natural symplectic structure, given by the wedge product on the de Rham cohomology, the structure group of the bundles $\mathcal{H}_{\kappa}^{1}(M, \mathbb{R})$ can be reduced to $S p(2 g, \mathbb{R}) \subset \mathrm{GL}(2 g, \mathbb{R})$. In this case Lyapunov exponents form a symmetric subset of the real line. Kontsevich and Zorich conjectured in [38], on the basis of numerical computations (see also [79]), that the Lyapunov spectrum is simple, that is:

Kontsevich-Zorich Conjecture. The $2 g$ Lyapunov exponents of the Kontsevich-Zorich cocycle have the following properties:

$$
1=\lambda_{1}>\lambda_{2}>\cdots>\lambda_{g}>0>\lambda_{g+1}=-\lambda_{g}>\cdots>\lambda_{2 g}=-\lambda_{1}=-1 .
$$

Remark 1.2. In [66] W. Veech proved that the Teichmüller geodesic flow is non-uniformly hyperbolic on each stratum $\mathcal{M}_{\kappa}^{(1)}$. Since the bundle $\mathcal{H}_{\kappa}^{1}(M, \mathbb{R})$ does not coincide with the whole tangent bundle of $\mathcal{M}_{\kappa}^{(1)}$, Veech's theorem does not imply the Kontsevich-Zorich Conjecture. By (1.1) the Lyapunov exponents of the Teichmüller geodesic flow can be written as follows, in terms of the 
exponents (1.2) of the Kontsevich-Zorich cocycle [78, §5], [38, §7]:

$$
\begin{aligned}
2 & \geq 1+\lambda_{2} \geq 1+\lambda_{3} \geq \cdots \geq 1+\lambda_{g} \geq \underbrace{1=\cdots=1}_{\sigma_{\kappa}-1} \\
& \geq 1-\lambda_{g} \geq \cdots \geq 1-\lambda_{2} \geq 0 \geq-1+\lambda_{2} \geq \cdots \geq-1+\lambda_{g} \\
& \geq \underbrace{-1=\cdots=-1}_{\sigma_{\kappa}-1} \geq-1-\lambda_{g} \geq-1-\lambda_{g-1} \geq \cdots \geq-1-\lambda_{2} \geq-2 .
\end{aligned}
$$

The non-uniform hyperbolicity of the Teichmüller flow is therefore equivalent to the statement that $\lambda_{2}<\lambda_{1}=1$.

In this paper we prove that the Kontsevich-Zorich cocycle is non-uniformly hyperbolic, that is $\lambda_{g}>0$. Our method also yields a new proof of Veech's theorem $\lambda_{2}<1$ (Corollary 2.2).

\section{Variational formulas}

Let $q \in \mathcal{M}_{\kappa}^{(1)}$. Any cohomology class $c \in H^{1}\left(M_{q}, \mathbb{R}\right)$ can be represented by a harmonic differential. In fact $c=\left[\Re\left(h^{+}\right)\right]$, where $h^{+}$is a holomorphic differential on $M_{q}$. The vector space $H^{1}\left(M_{q}, \mathbb{R}\right)$ can be endowed with the Hodge norm induced by the Hermitian structure on $M_{q}$. The KontsevichZorich cocycle along the Teichmüller orbit of $q$ can be written in the Hodge representation in the form of an O.D.E. on a Hilbert space of square integrable functions. We recall the basic setting established in [18].

Let $R_{q}:=|q|^{1 / 2}$ be the flat (smooth) metric induced by $q$ on $M$, degenerate at $\Sigma_{q}$, and let $\omega_{q}$ be the corresponding area form [58, 55.3$]$. At any point $p \in M_{q} \backslash \Sigma_{q}$, there exists a canonical holomorphic coordinate $z:=x+i y$ such that $q=d z^{2}[58, \S 5.1]$. Consequently,

$$
R_{q}=\left(d x^{2}+d y^{2}\right)^{1 / 2} \quad \text { and } \quad \omega_{q}=d x \wedge d y .
$$

At a zero $p \in \Sigma_{q}$ of order $k, q=z^{k} d z^{2}$ with respect to a canonical coordinate. Hence,

$$
R_{q}=|z|^{k / 2}\left(d x^{2}+d y^{2}\right)^{1 / 2} \text { and } \omega_{q}=|z|^{k} d x \wedge d y .
$$

Let $\mathcal{F}_{ \pm q}$ be the horizontal and the vertical foliation of $q$ respectively, defined by $\mathcal{F}_{q}:=\left\{\Im\left(q^{1 / 2}\right)=0\right\}, \mathcal{F}_{-q}:=\left\{\Re\left(q^{1 / 2}\right)=0\right\}$. Such foliations are naturally measured foliations in the sense of Thurston [62] with the transverse measures $\left|\Im\left(q^{1 / 2}\right)\right|,\left|\Re\left(q^{1 / 2}\right)\right|$ respectively. There exists a unique positively oriented frame $\{S, T\}$ of the tangent bundle $T M$ on $M \backslash \Sigma_{q}$, orthonormal with respect to $R_{q}$, such that $S, T$ are tangent to the horizontal, respectively the vertical, foliation 
of $q$. The vector fields $S, T$ are smooth on $M \backslash \Sigma_{q}$ and blow up at $\Sigma_{q}$. Let

$$
\begin{aligned}
& \eta_{T}:=\Re\left(q^{1 / 2}\right)=-\imath_{T} \omega_{q}, \\
& \eta_{S}:=\Im\left(q^{1 / 2}\right)=\imath_{S} \omega_{q} .
\end{aligned}
$$

Then $\omega_{q}=\eta_{T} \wedge \eta_{S}$ and, in addition, since $\eta_{S}$ and $\eta_{T}$ are closed 1-forms, the vector fields $S, T$ preserve the area form $\omega_{q}$.

Let $L_{q}^{2}(M):=L^{2}\left(M, \omega_{q}\right)$ be the Hilbert space of square-integrable functions with respect to the area form. The Cauchy-Riemann operators $\partial_{q}^{ \pm}:=$ $S \pm i T$ are closed on the domain $H^{1}(M) \subset L_{q}^{2}(M)$ (the Sobolev space of weakly differentiable functions with $L^{2}$ derivatives on the compact manifold $M)$ and have closed ranges $R_{q}^{ \pm}$of finite codimension. In fact, the adjoints of the Cauchy-Riemann operators satisfy the identity $\left(\partial_{q}^{ \pm}\right)^{*}=-\partial_{q}^{\mp}$, hence, by Hilbert space theory, $R_{q}^{ \pm}$are the orthogonal complements of the kernels $\mathcal{M}_{q}^{\mp} \subset L_{q}^{2}(M)$, which consist of anti-meromorphic, respectively meromorphic, $L_{q}^{2}$ functions (with poles at $\Sigma_{q}$ ) [18, Prop. 3.2]. There exist therefore two orthogonal decompositions:

$$
L_{q}^{2}(M)=R_{q}^{-} \oplus^{\perp} \mathcal{M}_{q}^{+}=R_{q}^{+} \oplus^{\perp} \mathcal{M}_{q}^{-}
$$

and two associated (orthogonal) projections $\pi_{q}^{ \pm}: L_{q}^{2}(M) \rightarrow \mathcal{M}_{q}^{ \pm}$. The spaces $\mathcal{M}_{q}^{ \pm}$, endowed with the real Euclidean structure induced by the Hilbert structure on $L_{q}^{2}(M)$, are isomorphic to the cohomology $H^{1}\left(M_{q}, \mathbb{R}\right)$, endowed with the Hodge inner product determined by the metric $R_{q}$ (by the area form $\omega_{q}$ ). In fact, the $\mathbb{R}$-linear maps $c_{q}^{ \pm}: \mathcal{M}_{q}^{ \pm} \rightarrow H^{1}\left(M_{q}, \mathbb{R}\right)$ defined by

$$
\begin{aligned}
& c_{q}^{+}\left(m^{+}\right):=\left[\Re\left(m^{+} q^{1 / 2}\right)\right], \\
& c_{q}^{-}\left(m^{-}\right):=\left[\Re\left(m^{-} \bar{q}^{1 / 2}\right)\right]
\end{aligned}
$$

are isomorphisms of vector spaces. In addition

$$
\left\|c_{q}^{ \pm}\left(m^{ \pm}\right)\right\|_{q}^{2}:=\int_{M} c_{q}^{ \pm}\left(m^{ \pm}\right) \wedge * c_{q}^{ \pm}\left(m^{ \pm}\right)=\left|m^{ \pm}\right|_{0}^{2},
$$

where $\|\cdot\|_{q}$ is the Hodge norm on $H^{1}\left(M_{q}, \mathbb{R}\right.$ ) (the Hodge *-operator on $M_{q}$ is given by $\left.* \eta_{T}=\eta_{S}, * \eta_{S}=-\eta_{T}\right)$ and $|\cdot|_{0}$ the norm on $L_{q}^{2}(M)$. These assertions follow from the remark that, if $h^{ \pm}$is any holomorphic, respectively anti-holomorphic, differential on $M_{q}$, then $h^{+} / q^{1 / 2}, h^{-} / \bar{q}^{1 / 2}$ are respectively a meromorphic, an anti-meromorphic function with poles at $\Sigma_{q}$ which belong to $L_{q}^{2}(M)$. The fact that all real cohomology classes can be represented as the real part of a holomorphic (or anti-holomorphic) differential is a classical property of Riemann surfaces [13, III.2]. A computation similar to (2.5) shows that the symplectic form on $H^{1}\left(M_{q}, \mathbb{R}\right)$, given by the wedge product, can be written in $\mathcal{M}_{q}^{ \pm}$as follows:

$$
c_{q}^{ \pm}\left(m_{1}^{ \pm}\right) \wedge c_{q}^{ \pm}\left(m_{2}^{ \pm}\right)=\Im\left(m_{1}^{ \pm}, m_{2}^{ \pm}\right)_{q},
$$

where $(\cdot, \cdot)_{q}$ is the inner product in $L_{q}^{2}(M)$. 
By (2.3) any function $u \in L_{q}^{2}(M)$ has a decomposition $u=\partial_{q}^{+} v+\pi_{q}^{-}(u)$ with $v \in H^{1}(M)$. The operator $U_{q}: L_{q}^{2}(M) \rightarrow L_{q}^{2}(M)$ given by

$$
\begin{aligned}
U_{q}(u): & =\partial_{q}^{-} v-\overline{\pi_{q}^{-}(u)}, \\
\text { if } \quad u & =\partial_{q}^{+} v+\pi_{q}^{-}(u)
\end{aligned}
$$

is a $\mathbb{R}$-linear isometry. In fact, by [18, Prop. 3.2] and the orthogonality of the decomposition (2.3), we have

$$
\left|U_{q}(u)\right|_{0}^{2}=\left|\partial_{q}^{-} v\right|_{0}^{2}+\left|\pi_{q}^{-}(u)\right|_{0}^{2}=\left|\partial_{q}^{+} v\right|_{0}^{2}+\left|\pi_{q}^{-}(u)\right|_{0}^{2}=|u|_{0}^{2} .
$$

Let $q_{t}:=G_{t}(q)$ denote the orbit of the quadratic differential $q \in Q_{\kappa}^{(1)}$ under the Teichmüller geodesic flow $G_{t}$. By the definition of the Teichmüller flow, $q_{t}$ is given by the equations:

$$
\begin{aligned}
& \eta_{T}(t):=\Re\left(q_{t}^{1 / 2}\right)=e^{t} \Re\left(q^{1 / 2}\right)=e^{t} \eta_{T}, \\
& \eta_{S}(t):=\Im\left(q_{t}^{1 / 2}\right)=e^{-t} \Im\left(q^{1 / 2}\right)=e^{-t} \eta_{S} .
\end{aligned}
$$

In all the arguments which follow it is crucial that the area form $\omega_{q}$ of the metric $R_{q}$ and, consequently, the inner product of the Hilbert space $L_{q}^{2}(M)$, are invariant under the Teichmüller geodesic flow. Let $\left\{S_{t}, T_{t}\right\}$ and $\partial_{t}^{ \pm}:=S_{t} \pm i T_{t}$ be the orthonormal system and the Cauchy-Riemann operators determined by $q_{t}$. We have:

$$
\partial_{t}^{ \pm}=S_{t} \pm i T_{t}=e^{-t} S \pm i e^{t} T .
$$

Let $\mathcal{M}_{t}^{ \pm}:=N\left(\partial_{t}^{ \pm}\right) \subset L_{q}^{2}(M)$ denote the subspaces of meromorphic, respectively anti-meromorphic, $L_{q}^{2}$ functions on the Riemann surface $M_{t}$ (endowed with the complex structure determined by $q_{t}$ ). The Kontsevich-Zorich cocycle $G_{t}^{K Z}$ is described, along the orbit $q_{t}:=G_{t}(q)$ of the Teichmüller flow, by the following variational formula:

LEmma 2.1. The ordinary differential equation

$$
u^{\prime}=U_{q_{t}}(u)
$$

is well-defined in $L_{q}^{2}(M)$ and satifies the following properties.

(1) Solutions of the Cauchy problem for (2.8) exist for all times and are uniquely determined by the initial condition.

(2) If $u_{t} \in L_{q}^{2}(M)$ is any solution of (2.8) with initial condition $u_{0} \in \mathcal{M}_{q}^{+}$, then $u_{t} \in \mathcal{M}_{t}^{+}$, for all $t \in \mathbb{R}$.

(3) Let $m_{t}^{+} \in \mathcal{M}_{t}^{+}$be the unique solution of (2.8) with initial condition $m_{0}^{+}=$ $m^{+} \in \mathcal{M}_{q}^{+}$. For all $t \in \mathbb{R}$, we have

$$
G_{t}^{K Z}\left(c_{q}^{+}\left(m^{+}\right)\right)=c_{q_{t}}^{+}\left(m_{t}^{+}\right)
$$


Proof of (1). The O.D.E. (2.8) is well defined since the Hilbert space $L_{q}^{2}(M)$ is invariant along orbits of the Teichmüller flow. The function $F(t, u):=$ $U_{q_{t}}(u)$ is uniformly Lipschitz in the second coordinate, since $U_{q}$ is an isometry, and smooth on $\mathbb{R} \times L_{q}^{2}(M)$. In fact, the Cauchy-Riemann operators $\partial_{t}^{ \pm}$, densely defined on the Hilbert space $L_{q}^{2}(M)$ with common domain $H^{1}(M)$, depend smoothly on $t \in \mathbb{R}$, as it can be seen by explicitly writing them in terms of the fixed orthonormal frame $\{S, T\}$. The standard local existence and uniqueness results for O.D.E. on Banach spaces therefore apply [40, Chap. IV]. Since for any solution $\left|u_{t}^{\prime}\right|_{0}=\left|u_{t}\right|_{0}$, it follows that

$$
\left|u_{t}\right|_{0} \leq\left|u_{0}\right|_{0}+\int_{0}^{t}\left|u_{s}\right|_{0} d s
$$

hence by Gronwall's lemma $\left|u_{t}\right|_{0}$ does not blow up in finite time. Local solutions can be therefore continued for all times.

Proof of (2). A computation shows that the flow of (2.8) preserves meromorphic functions. In fact, since by $(2.7)$ and $\left(2.7^{\prime}\right)$

$$
\frac{d}{d t} q_{t}^{1 / 2}=\bar{q}^{1 / 2}, \quad \frac{d}{d t} \partial_{t}^{ \pm}=-\partial_{t}^{\mp},
$$

it follows that, if $u_{t}$ is a solution of the O.D.E. (2.8), we have:

$$
\frac{d}{d t}\left(\partial_{t}^{+} u_{t}\right)=-\partial_{t}^{-}\left(u_{t}\right)+\partial_{t}^{+}\left(u_{t}^{\prime}\right)=0
$$

in the weak sense in $L_{q}^{2}(M)$.

Proof of (3). Let $\pi_{t}^{ \pm}: L_{q}^{2}(M) \rightarrow \mathcal{M}_{t}^{ \pm} \subset L_{q}^{2}(M)$ be the orthogonal projections. If $m_{t}^{+} \in \mathcal{M}_{t}^{+}$is a solution of (2.8), there exists a function $v_{t} \in H^{1}(M)$ (unique up to additive constants) such that:

$$
\begin{aligned}
m_{t}^{+} & =\partial_{t}^{+} v_{t}+\pi_{t}^{-}\left(m_{t}^{+}\right), \\
\frac{d}{d t} m_{t}^{+} & =\partial_{t}^{-} v_{t}-\overline{\pi_{t}^{-}\left(m_{t}^{+}\right)} .
\end{aligned}
$$

If $v_{t}$ is chosen with zero average for all $t \in \mathbb{R}$ then the map $t \rightarrow v_{t} \in H^{1}(M)$ is smooth. By (2.12)

$$
\frac{d}{d t} \Re\left(m_{t}^{+} q_{t}^{1 / 2}\right)=\Re\left(\left(\frac{d}{d t} m_{t}^{+}+\overline{m_{t}^{+}}\right) q_{t}^{1 / 2}\right)=2 \Re\left(d v_{t}\right) \equiv 0 \in H^{1}(M, \mathbb{R}) .
$$

Hence $\left(2.8^{\prime}\right)$ follows by the definition of the Kontsevich-Zorich cocycle $G_{t}^{K Z}$. In fact, over the Teichmüller space, the cocycle acts as the identity on cohomology classes. 
Let $c \in H^{1}\left(M_{q}, \mathbb{R}\right)$ and $c_{t}:=G_{t}^{K Z}(c)$. Let $c_{t}=\left[\Re\left(m_{t}^{+} q_{t}^{1 / 2}\right)\right], m_{t}^{+} \in \mathcal{M}_{t}^{+}$. By Lemma 2.1, $m_{t}^{+}$is a solution of (2.8) or, equivalently, of (2.12).

Lemma 2.1'. The evolution of the Hodge norm under the KonsevichZorich cocycle is described by the following formulas:

$$
\begin{aligned}
& \frac{d}{d t}\left|m_{t}^{+}\right|_{0}^{2}=-2 \Re B_{q}\left(m_{t}^{+}\right):=-2 \Re \int_{M}\left(m_{t}^{+}\right)^{2} \omega_{q}, \\
& \frac{d^{2}}{d t^{2}}\left|m_{t}^{+}\right|_{0}^{2}=4\left\{\left|\pi_{t}^{-}\left(m_{t}^{+}\right)\right|_{0}^{2}-\Re \int_{M}\left(\partial_{t}^{+} v_{t}\right)\left(\partial_{t}^{-} v_{t}\right) \omega_{q}\right\} .
\end{aligned}
$$

Proof. By (2.5) and the invariance of the $L_{q}^{2}$ inner product under the Teichmüller flow, $\left\|c_{t}\right\|=\left|m_{t}^{+}\right|_{0}$. A computation, based on (2.12), shows that

$$
\begin{aligned}
\frac{d}{d t}\left|m_{t}^{+}\right|_{0}^{2} & =2 \Re\left(m_{t}^{+}, \frac{d}{d t} m_{t}^{+}\right)_{q}=-\Re\left(m_{t}^{+}, \overline{\pi_{t}^{-}\left(m_{t}^{+}\right)}\right)_{q} \\
& =-2 \Re\left(m_{t}^{+}, \overline{m_{t}^{+}}\right)_{q}=-2 \Re \int_{M}\left(m_{t}^{+}\right)^{2} \omega_{q}, \\
\frac{d}{d t} B_{q}\left(m_{t}^{+}\right) & =2 \int_{M} m_{t}^{+} \frac{d}{d t} m_{t}^{+} \omega_{q} \\
& =2 \int_{M}\left(\partial_{t}^{+} v_{t}+\pi_{t}^{-}\left(m_{t}^{+}\right)\right)\left(\partial_{t}^{-} v_{t}-\overline{\pi_{t}^{-}\left(m_{t}^{+}\right)}\right) \omega_{q} \\
& =-2\left|\pi_{t}^{-}\left(m_{t}^{+}\right)\right|_{0}^{2}+2 \int_{M}\left(\partial_{t}^{+} v_{t}\right)\left(\partial_{t}^{-} v_{t}\right) \omega_{q} .
\end{aligned}
$$

Calculations of first and second variation formulas for various functionals on the Teichmüller space are widespread in Teichmüller theory (see for instance [64], [72], [60], [61]). In particular, M.Taniguchi [60] proved variational formulas for the extremal length of the homology class of a given curve (equivalently, for the Hodge norm of the dual cohomology class), with respect to fairly general quasi-conformal deformations. In the special case of Teichmüller deformations, not explicitly considered in [60], [61], Taniguchi's variational formulas agree, by Lemma 2.1 in the case of the second variation, with (2.14) (and (3.12) below). We thank the referee and M.Wolf for bringing the above mentioned references to our attention.

Lemma 2.1' immediately implies Veech's result, recalled in Remark 1.2. In fact, we will obtain an upper bound for the second Lyapunov exponent of the Kontsevich-Zorich cocycle in terms of the function $\Lambda^{+}: \mathcal{M}_{\kappa}^{(1)} / S O(2, \mathbb{R}) \rightarrow \mathbb{R}$, defined as follows:

$$
\Lambda^{+}(q):=\max \left\{\frac{\left|B_{q}\left(m^{+}\right)\right|}{\left|m^{+}\right|_{0}^{2}} \mid m^{+} \in \mathcal{M}_{q}^{+} \backslash\{0\}, \int_{M} m^{+} \omega_{q}=0\right\} .
$$


COROLlary 2.2. Let $\mu$ be any $G_{t^{-}}$ergodic probability measure on $\mathcal{M}_{\kappa}^{(1)}$. The second Lyapunov exponent of the Kontsevich-Zorich cocycle with respect to the measure $\mu$ satisfies the following inequality:

$$
\lambda_{2}^{\mu} \leq \int_{\mathcal{M}_{\kappa}^{(1)}} \Lambda^{+} d \mu<\lambda_{1}^{\mu}=1 .
$$

Proof. The Kontsevich-Zorich cocycle has two 1-dimensional invariant continuous sub-bundles $E_{ \pm 1} \subset \mathcal{H}_{\kappa}^{1}(M, \mathbb{R})$ with Lyapunov exponents \pm 1 . At any $q \in \mathcal{M}_{\kappa}^{(1)}$

$$
\begin{aligned}
E_{1}(q) & :=\mathbb{R} \cdot\left[\Im\left(q^{1 / 2}\right)\right], \\
E_{-1}(q) & :=\mathbb{R} \cdot\left[\Re\left(q^{1 / 2}\right)\right] .
\end{aligned}
$$

It follows in particular that, for all $G_{t}$-ergodic probability measures $\mu, \lambda_{1}^{\mu}=1$, $\lambda_{2 g}^{\mu}=-\lambda_{1}^{\mu}=-1$. Let $E_{0} \subset \mathcal{H}_{\kappa}^{1}(M, \mathbb{R})$ be the continuous invariant sub-bundle defined as follows. At any $q \in \mathcal{M}_{\kappa}^{(1)}$,

$$
E_{0}(q):=\left\{c \in H^{1}\left(M_{q}, \mathbb{R}\right) \mid c \wedge\left[\Re\left(q^{1 / 2}\right)\right]=c \wedge\left[\Im\left(q^{1 / 2}\right)\right]=0\right\} .
$$

A cohomology class $c=\left[\Re\left(m^{+} q^{1 / 2}\right)\right] \in E_{0}(q)$ if and only if $\int_{M} m^{+} \omega_{q}=0$. There is an invariant continuous splitting $\mathcal{H}_{\kappa}^{1}(M, \mathbb{R})=E_{1} \oplus E_{-1} \oplus E_{0}$. In addition, the bundle $E_{0}$ is invariant under the action of the circle group $S O(2, \mathbb{R})$ on $\mathcal{M}_{\kappa}^{(1)}$. Let $c \in E_{0}(q)$. Let $q_{t}:=G_{t}(q)$ and $c_{t}:=G_{t}^{K Z}(c)$. Then $c_{t}=\left[\Re\left(m_{t}^{+} q_{t}\right)^{1 / 2}\right]$, where $m_{t}^{+} \in \mathcal{M}_{t}^{+}$has zero average. Hence, by Lemma $2.1^{\prime}$,

$$
\frac{d}{d t} \log \left|m_{t}^{+}\right|_{0}=-\frac{\Re B_{q}\left(m_{t}^{+}\right)}{\left|m_{t}^{+}\right|_{0}^{2}} \leq \Lambda^{+}\left(q_{t}\right) .
$$

By taking time averages, it follows that

$$
\frac{1}{\mathcal{T}} \log \frac{\left|m_{\mathcal{T}}^{+}\right|_{0}}{\left|m^{+}\right|_{0}} \leq \frac{1}{\mathcal{T}} \int_{0}^{\mathcal{T}} \Lambda^{+}\left(q_{t}\right) d t
$$

By Oseledec's theorem [49], [32, Th. S.2.9], and Birkhoff's ergodic theorem (for the ergodic measure $\mu$ ), by taking the limit in $\left(2.19^{\prime}\right)$ as $\mathcal{T} \rightarrow+\infty$, we obtain the first of the two inequalities in (2.17). In order to prove the remaining inequality we will show that $\Lambda^{+}(q)<1$ for all $q \in \mathcal{M}_{\kappa}^{(1)}$. By Schwartz inequality, we have:

$$
\left|B_{q}\left(m^{+}\right)\right|=\left|\left(m^{+}, \overline{m^{+}}\right)_{q}\right| \leq\left|m^{+}\right|_{0}^{2},
$$

where equality holds if and only if there exists $\lambda \in \mathbb{C}(|\lambda|=1)$ such that $\overline{m^{+}}=\lambda m^{+}$. However, in that case $m^{+}$would be meromorphic and antimeromorphic, hence constant, and, having zero average, $m^{+}=0$. It follows that, if $m^{+} \neq 0$, the inequality in $(2.20)$ is strict, hence, by compactness of the unit sphere in $\mathcal{M}_{q}^{+} \subset L_{q}^{2}(M), \Lambda^{+}(q)<1$. 


\section{A lower bound for the second Lyapunov exponent}

The Teichmüller space $Q_{g}^{(1)}$ is foliated by the orbits of the action of $\mathrm{SL}(2, \mathbb{R}) \subset \mathrm{GL}_{+}(2, \mathbb{R})$ described in Section 1 . The leaves are 3 -dimensional and are isometric to the unit cotangent bundle $S^{*}(D) \equiv \mathrm{SL}(2, \mathbb{R})$ of the Poincaré disk $D:=D(0,1)$ (endowed with the hyperbolic metric $|d z| /\left(1-|z|^{2}\right)$ of constant curvature -4$)$. The circle group $\mathrm{SO}(2, \mathbb{R})$ is a subgroup of $\operatorname{SL}(2, \mathbb{R})$ and acts therefore on $Q_{g}^{(1)}$. The quotient $Q_{g}^{(1)} / \mathrm{SO}(2, \mathbb{R})$ is then foliated by 2dimensional leaves isometric to the Poincaré disk. In fact, given any $q \in Q_{g}^{(1)}$, there exists an isometric embedding $j_{q}: D \rightarrow Q_{g}^{(1)} / \mathrm{SO}(2, \mathbb{R})$ such that $j_{q}(0)=$ $[q] \in Q_{g}^{(1)} / \mathrm{SO}(2, \mathbb{R})$. Let $q_{\theta}:=e^{\imath \theta} q$ be the rotation of the quadratic differential $q$ by an angle $\theta \in[0,2 \pi]$ and, if $z=r e^{\imath \theta}$, let

$$
q_{z}:=G_{t}\left(q_{\theta}\right), \quad t:=\frac{1}{2} \log \frac{1+r}{1-r} .
$$

The embedding $j_{q}$ is given by

$$
j_{q}(z):=\left[q_{z}\right] \in Q_{g}^{(1)} / \mathrm{SO}(2, \mathbb{R}), \quad z \in D .
$$

Let $\pi_{g}: Q_{g}^{(1)} \rightarrow T_{g}$ be the canonical projection onto the Teichmüller space $T_{g}$ of (marked) Riemann surfaces, defined by $\pi_{g}(q)=M_{q}$, the Riemann surface carrying the holomorphic quadratic differential $q \in Q_{g}^{(1)}$. The composed map $\pi_{g} \circ j_{q}$ is an isometric embedding and its image is called a Teichmüller disk [46, $\S 2.6 .5]$. Since the action of $\mathrm{GL}_{+}(2, \mathbb{R})$ commutes with the action of the mapping class group $\Gamma_{g}$, the foliation of the space $Q_{g}^{(1)} / \mathrm{SO}(2, \mathbb{R})$ by Teichmüller disks projects to a foliation $\mathcal{T}_{g}$ of $\mathcal{M}_{g}^{(1)} / \mathrm{SO}(2, \mathbb{R})$. Any leaf $\mathcal{T}$ of $\mathcal{T}_{g}$ is a complex curve endowed with a hyperbolic metric of constant curvature -4 . It is in fact the quotient of the Poincaré disk by a discrete group of isometries. The generic leaf is isometric to the Poincaré disk [38, §7]. The foliated unit cotangent bundle $S^{*}\left(\mathcal{T}_{g}\right)$ gives a foliation of the moduli space $\mathcal{M}_{g}^{(1)}$, which can be naturally identified with the orbit foliation of the action of $\mathrm{SL}(2, \mathbb{R})$. The Teichmüller geodesic flow preserves the foliation $S^{*}\left(\mathcal{T}_{g}\right)$ (since the latter is the orbit foliation of the full group $\operatorname{SL}(2, \mathbb{R})$ ) and its restriction to each leaf $S^{*}(\mathcal{T})$ coincides with the geodesic flow of the Poincaré metric on $\mathcal{T}$. The foliations $\mathcal{T}_{g}$ and $S^{*}\left(\mathcal{T}_{g}\right)$ preserve each stratum $\mathcal{M}_{\kappa}^{(1)} / \mathrm{SO}(2, \mathbb{R})$ and $\mathcal{M}_{\kappa}^{(1)}$ respectively.

In [38] M. Kontsevich and A. Zorich outlined the proof of a formula for the sum $\lambda_{1}+\ldots+\lambda_{g}$ of the non-negative Lyapunov exponents of the Kontsevich-Zorich cocycle, based on the computation on a Teichmüller disk of the (Poincaré) Laplacian of the logarithm of the volume on a generic Lagrangian subspace of the fiber $H^{1}(M, \mathbb{R})$. A new version of their formula will be proved in Section 5. Following [38], the basic tool in proving bounds for the 
Lyapunov exponents of the Kontsevich-Zorich cocycle is given by the elementary lemma below, which does not rely on Sullivan's results on the Brownian motion (on the Poincaré disk) [59] exploited in [38]. We remark that a similar idea of using sub-harmonic functions to prove lower bounds for Lyapunov exponents was introduced, in a different context, by M. Herman [26].

Lemma 3.1. Let $\triangle_{h}$ be the (hyperbolic) Laplacian on the Poincaré disk $D$ and let $L: D \rightarrow \mathbb{R}$ be a smooth solution of the Poisson equation $\triangle_{h} L=\Lambda$, where $\Lambda$ is a smooth bounded function on $D$. Then

$$
\frac{1}{2 \pi} \frac{\partial}{\partial t} \int_{0}^{2 \pi} L(t, \theta) d \theta=\frac{1}{2} \tanh (t) \frac{1}{\left|D_{t}\right|} \int_{D_{t}} \Lambda \omega_{P}
$$

where $(t, \theta)$ are geodesic polar coordinates on the Poincaré disk, $\left|D_{t}\right|$ denotes the Poincaré area of the disk $D_{t}$ of geodesic radius $t>0$, centered at the origin, and $\omega_{P}$ is the Poincaré area form.

Proof. In geodesics polar coordinates centered at the origin, the Poincaré Laplacian can be written as follows [24, Intr. §4.2]:

$$
\triangle_{h}=\frac{\partial^{2}}{\partial t^{2}}+2 \operatorname{coth}(2 t) \frac{\partial}{\partial t}+\frac{4}{\sinh ^{2}(2 t)} \frac{\partial^{2}}{\partial \theta^{2}} .
$$

Let $L_{r}, \Lambda_{r}$ be the circular averages of the functions $L, \Lambda$ respectively:

$$
L_{r}(t):=\frac{1}{2 \pi} \int_{0}^{2 \pi} L(t, \theta) d \theta \quad, \quad \Lambda_{r}(t):=\frac{1}{2 \pi} \int_{0}^{2 \pi} \Lambda(t, \theta) d \theta .
$$

By averaging the equation $\triangle_{h} L=\Lambda$, we obtain the following O.D.E.:

$$
\frac{\partial^{2}}{\partial t^{2}} L_{r}+2 \operatorname{coth}(2 t) \frac{\partial}{\partial t} L_{r}=\Lambda_{r}
$$

The equation $\left(3.4^{\prime}\right)$ can be explicitly solved by setting $M_{r}:=\partial L_{r} / \partial t$. Since $M_{r}(0)=0$ the solution has the following expression:

$$
M_{r}(t)=\frac{1}{\sinh (2 t)} \int_{0}^{t} \Lambda_{r}(s) \sinh (2 s) d s .
$$

We recall the following elementary formulas from hyperbolic geometry:

$$
\omega_{P}=\frac{1}{2} \sinh (2 t) d t d \theta \quad, \quad\left|D_{t}\right|=\pi \frac{\cosh (2 t)-1}{2} .
$$

Hence, by (3.5) and (3.6),

$$
\frac{\partial}{\partial t} L_{r}=\frac{\cosh (2 t)-1}{2 \sinh (2 t)} \frac{1}{\left|D_{t}\right|} \int_{D_{t}} \Lambda \omega_{P}=\frac{1}{2} \tanh (t) \frac{1}{\left|D_{t}\right|} \int_{D_{t}} \Lambda \omega_{P}
$$

Let $q \in Q_{\kappa}^{(1)}, M_{z}$ be the Riemann surface carrying the quadratic differential $q_{z}$, given by (3.1), and let $\mathcal{M}_{z}^{ \pm}$be the spaces of meromorphic, respectively anti-meromorphic, functions on $M_{z}$ which belong to $L_{q}^{2}(M)$. We remark 
that the $L^{2}$ structure induced by $q_{z}$ is independent of $z \in D$, since the area form of a quadratic differential is invariant under the action of $\operatorname{SL}(2, \mathbb{R})$. Let $\pi_{z}^{ \pm}: L_{q}^{2}(M) \rightarrow \mathcal{M}_{z}^{ \pm}$denote the orthogonal projections. Let $c \in H^{1}\left(M_{q}, \mathbb{R}\right)$. There exists a uniquely determined meromorphic function $m_{z}^{+} \in \mathcal{M}_{z}^{+}$such that $c=\left[\Re\left(m_{z}^{+} q_{z}^{1 / 2}\right)\right]$. The function $z \rightarrow m_{z}^{+} \in L_{q}^{2}(M)$ is holomorphic on $D$, but it depends on the choice of the base point $q \in Q_{\kappa}^{(1)}$. The norm $\left|m_{z}^{+}\right|_{0}$ is invariant under the action of the circle group on $Q_{\kappa}^{(1)}$, hence it gives a well defined real analytic function on the Teichmüller disk $j_{q}(D) \subset Q_{g}^{(1)} / \mathrm{SO}(2, \mathbb{R})$, independent of the choice of the base point.

LEMMA 3.2. The following formula holds:

$$
\triangle_{h} \log \left|m_{z}^{+}\right|_{0}=4 \frac{\left|\pi_{z}^{-}\left(m_{z}^{+}\right)\right|_{0}^{2}}{\left|m_{z}^{+}\right|_{0}^{2}}-2 \frac{\left|B_{q}\left(m_{z}^{+}\right)\right|^{2}}{\left|m_{z}^{+}\right|_{0}^{4}} \geq 2 \frac{\left|\pi_{z}^{-}\left(m_{z}^{+}\right)\right|_{0}^{2}}{\left|m_{z}^{+}\right|_{0}^{2}}
$$

Proof. If $\left[q^{\prime}\right] \in j_{q}(D)$, then $j_{q^{\prime}}(D)=j_{q}(D)$. Hence it suffices to prove the formula at the origin $z=0$. Let $c=\left[\Re\left(m^{+} q^{1 / 2}\right)\right] \in H^{1}(M, \mathbb{R})$, where $m^{+} \in \mathcal{M}_{q}^{+}$. Let $q_{\theta}:=e^{\imath \theta} q$ and $q_{(t, \theta)}:=G_{t}\left(q_{\theta}\right)$. Let $X_{\theta}$ be the normalized directional derivative at $z=0$ defined, for any smooth function $\phi: D \rightarrow \mathbb{R}$, by

$$
X_{\theta} \phi:=\left.\frac{d}{d r} \phi\left(r e^{\imath \theta}\right)\right|_{r=0} .
$$

By Lemma 2.1' we have:

$$
\begin{aligned}
& X_{\theta}\left|m_{z}^{+}\right|_{0}^{2}=-2 \Re\left[e^{-\imath \theta} B_{q}\left(m^{+}\right)\right]=-2 \Re\left[e^{-\imath \theta} \int_{M}\left(m^{+}\right)^{2} \omega_{q}\right], \\
& X_{\theta}^{2}\left|m_{z}^{+}\right|_{0}^{2}=4\left\{\left|\pi_{q}^{-}\left(m^{+}\right)\right|_{0}^{2}-\Re\left[e^{-2 \imath \theta} \int_{M}\left(\partial_{q}^{+} v\right)\left(\partial_{q}^{-} v\right) \omega_{q}\right]\right\} .
\end{aligned}
$$

In fact, let $\mathcal{M}_{\theta}^{ \pm} \subset L_{q}^{2}(M)$ be the kernels of the Cauchy-Riemann operators $\partial_{\theta}^{ \pm}$determined by the quadratic differential $q_{\theta}$. Let $\pi_{\theta}^{ \pm}: L_{q}^{2}(M) \rightarrow \mathcal{M}_{\theta}^{ \pm}$ be the orthogonal projections. Any cohomology class $c \in H^{1}(M, \mathbb{R})$ can be represented as $c=\left[\Re\left(m_{\theta}^{+} q_{\theta}^{1 / 2}\right)\right]$, where $m_{\theta}^{+} \in \mathcal{M}_{\theta}^{+}$. We have:

$$
\begin{aligned}
\partial_{\theta}^{ \pm} & =e^{ \pm \imath \frac{\theta}{2}} \partial_{q}^{ \pm}, \\
\pi_{\theta}^{ \pm} & =\pi_{q}^{ \pm}, \\
m_{\theta}^{+} & =e^{-\imath \frac{\theta}{2}} m^{+},
\end{aligned}
$$

hence, in particular, $\mathcal{M}_{\theta}^{ \pm}=\mathcal{M}_{q}^{ \pm}$and, if $m^{+}=\partial_{q}^{+} v+\pi_{q}^{-}\left(m^{+}\right)$,

$$
\begin{aligned}
m_{\theta}^{+} & =\partial_{\theta}^{+} v_{\theta}+\pi_{\theta}^{-}\left(m_{\theta}^{+}\right), \\
v_{\theta} & =e^{-\imath \theta} v .
\end{aligned}
$$

The formulas (3.10) follow therefore from the formulas (2.14), taken at $t=0$, for the quadratic differential $q_{\theta}$. 
The hyperbolic gradient and the hyperbolic Laplacian can be written in terms of the directional derivatives $X_{\theta}$. Hence, by (3.10), at $z=0$,

$$
\begin{aligned}
& \nabla_{h}\left|m_{z}^{+}\right|_{0}^{2}=\left(X_{0}\left|m_{z}^{+}\right|_{0}^{2}, X_{\frac{\pi}{2}}\left|m_{z}^{+}\right|_{0}^{2}\right)=-2\left(\Re B_{q}\left(m^{+}\right), \Im B_{q}\left(m^{+}\right)\right), \\
& \triangle_{h}\left|m_{z}^{+}\right|_{0}^{2}=\frac{1}{\pi} \int_{0}^{2 \pi} X_{\theta}^{2}\left|m_{z}^{+}\right|_{0}^{2} d \theta=8\left|\pi_{q}^{-}\left(m^{+}\right)\right|_{0}^{2} .
\end{aligned}
$$

Since, given any positive smooth function $\phi$ on $D$,

$$
\triangle_{h} \log \phi=\frac{1}{2}\left\{\frac{\triangle_{h}\left(\phi^{2}\right)}{\phi^{2}}-\frac{\left|\nabla_{h} \phi^{2}\right|^{2}}{\phi^{4}}\right\}
$$

the identity in (3.8) at $z=0$ follows from (3.12) by taking $\phi(z):=\left|m_{z}^{+}\right|_{0}$ in (3.13). In addition, $\left|B_{q}\left(m_{z}^{+}\right)\right| \leq\left|\pi_{z}^{-}\left(m_{z}^{+}\right)\right|_{0}\left|m_{z}^{+}\right|_{0}$, since

$$
\left|B_{q}\left(m_{z}^{+}\right)\right|=\left|\left(m_{z}^{+}, \overline{m_{z}^{+}}\right)_{q}\right|=\left|\left(\pi_{z}^{-}\left(m_{z}^{+}\right), \overline{m_{z}^{+}}\right)_{q}\right| \leq\left|\pi_{z}^{-}\left(m_{z}^{+}\right)\right|_{0}\left|m_{z}^{+}\right|_{0} .
$$

The inequality in (3.8) follows from (3.14) and the proof is therefore complete.

The second Lyapunov exponent of the Kontsevich-Zorich cocycle, with respect to any $\operatorname{SL}(2, \mathbb{R})$-invariant ergodic measure, can be bounded from below in terms of the non-negative function $\Lambda^{-}: \mathcal{M}_{\kappa}^{(1)} / \mathrm{SO}(2, \mathbb{R}) \rightarrow \mathbb{R}$, defined as follows:

$$
\Lambda^{-}(q):=\min \left\{\frac{\left|\pi_{q}^{-}\left(m^{+}\right)\right|_{0}^{2}}{\left|m^{+}\right|_{0}^{2}} \mid m^{+} \in \mathcal{M}_{q}^{+} \backslash\{0\}\right\}
$$

THEOREM 3.3. Let $\mu$ be any $G_{t^{-}}$ergodic probability measure on $\mathcal{M}_{\kappa}^{(1)}$ such that for $\mu$-almost all orbits of the circle group the Haar (Lebesgue) measure is absolutely continuous with respect to the conditional measure induced by $\mu$. The second Lyapunov exponent $\lambda_{2}^{\mu}$ of the Kontsevich-Zorich cocycle, with respect to the measure $\mu$, satisfies the following lower bound:

$$
\lambda_{2}^{\mu} \geq \int_{\mathcal{M}_{\kappa}^{(1)}} \Lambda^{-} d \mu
$$

Proof. Since the Lebesgue measures on the orbits of the circle action are absolutely continuous with respect to the conditional measures induced by $\mu$, by Fubini's theorem, Birkhoff's ergodic theorem and Oseledec's theorem, for $\mu$-almost all $q \in \mathcal{M}_{\kappa}^{(1)}, q_{\theta}$ is a Birkhoff generic point and a regular point (in the sense of Oseledec's theorem) for almost all $\theta \in[0,2 \pi]$. The Lyapunov exponent $\lambda_{2}^{\mu}$ is the top Lyapunov exponent of the restriction of the Kontsevich-Zorich cocycle to the invariant sub-bundle $E_{0}$, introduced in the proof of Corollary 2.2. We recall that $E_{0}$ is invariant under the action of the circle group $\operatorname{SO}(2, \mathbb{R})$ and a cohomology class $c=\left[\Re\left(m^{+} q^{1 / 2}\right)\right] \in E_{0}(q)$ if and only if $\int_{M} m^{+} \omega_{q}=0$. 
Let $c \in E_{0}(q)$. Let $q_{z}$ be given by (3.1) and $m_{z}^{+} \in \mathcal{M}_{z}^{+}$be a (zero average) meromorphic function in $L_{q}^{2}(M)$ such that $c=\left[\Re\left(m_{z}^{+} q_{z}^{1 / 2}\right)\right]$. Let $G_{z}^{K Z}(c)$ be the cohomology class $c$ at the point $q_{z} \in Q_{\kappa}^{(1)}$ (the parallel transport of cohomology classes is trivial over the Teichmüller space $\left.Q_{\kappa}^{(1)}\right)$ and let $L: D \rightarrow \mathbb{R}$ be the smooth function $L(z):=\log \left\|G_{z}^{K Z}(c)\right\|=\log \left|m_{z}^{+}\right|_{0}$. Let $z \equiv(t, \theta)$ with respect to geodesic polar coordinates, that is $z=r e^{\imath \theta}, r=\tanh (t)$. By Lemmas 3.1, 3.2 and definition (3.15), we have:

$$
\frac{1}{2 \pi} \frac{\partial}{\partial t} \int_{0}^{2 \pi} \log \left\|G_{(t, \theta)}^{K Z}(c)\right\| d \theta \geq \tanh (t) \frac{1}{\left|D_{t}\right|} \int_{D_{t}} \Lambda^{-}\left(G_{s}\left(q_{\theta}\right)\right) \omega_{P}(s, \theta)
$$

Let $\sigma_{q}$ be the normalized canonical (Haar) measure on the unit sphere $E_{0}^{(1)}(q)$ of the Euclidean space $E_{0}(q) \subset H^{1}\left(M_{q}, \mathbb{R}\right)$, endowed with the Hodge inner product induced by the metric $R_{q}$. The measure $\sigma_{q}$ is invariant under the action of the circle group $\mathrm{SO}(2, \mathbb{R})$ on the space $Q_{g}$. By averaging the inequality in (3.17) over $c \in E_{0}^{(1)}(q)$ with respect to $\sigma_{q}$, then over the moduli space $\mathcal{M}_{\kappa}^{(1)}$ with respect to the $G_{t}$-invariant measure $\mu$, we obtain:

$$
\begin{aligned}
\frac{1}{2 \pi} \frac{\partial}{\partial t} \int_{\mathcal{M}_{\kappa}^{(1)}} \int_{E_{0}^{(1)}(q)} \int_{0}^{2 \pi} \log \left\|G_{(t, \theta)}^{K Z}(c)\right\| d \theta d \sigma_{q} d \mu & \\
& \geq \tanh (t) \int_{\mathcal{M}_{\kappa}^{(1)}} \Lambda^{-} d \mu
\end{aligned}
$$

Finally, by taking the time average of $\left(3.17^{\prime}\right)$ over the interval $[0, \mathcal{T}]$, we have

$$
\begin{aligned}
\frac{1}{2 \pi \mathcal{T}} \int_{\mathcal{M}_{\kappa}^{(1)}} \int_{E_{0}^{(1)}(q)} \int_{0}^{2 \pi} \log \left\|G_{(\mathcal{T}, \theta)}^{K Z}(c)\right\| d \theta d \sigma_{q} d \mu \\
\geq \frac{\log \cosh \mathcal{T}}{\mathcal{T}} \int_{\mathcal{M}_{\kappa}^{(1)}} \Lambda^{-} d \mu .
\end{aligned}
$$

Hence, by passing to the limit as $\mathcal{T} \rightarrow+\infty$ in (3.18), we obtain (3.16). In fact, since $c \in E_{0}$, by Oseledec's theorem,

$$
\lim _{\mathcal{T} \rightarrow+\infty} \frac{1}{2 \pi \mathcal{T}} \int_{\mathcal{M}_{\kappa}^{(1)}} \int_{E_{0}^{(1)}(q)} \int_{0}^{2 \pi} \log \left\|G_{(\mathcal{T}, \theta)}^{K Z}(c)\right\| d \theta d \sigma_{q} d \mu \leq \lambda_{2}^{\mu} .
$$

Proving that $\lambda_{2}^{\mu}>0$ requires a deeper analysis, carried out in the next section, of the projection operator $\pi_{q}^{-}: \mathcal{M}_{q}^{+} \rightarrow \mathcal{M}_{q}^{-}$, which enters crucially in the lower bound given by Theorem 3.3, as the quadratic differential varies over the moduli space. 


\section{The determinant locus}

Let $M$ be a marked Riemann surface of genus $g \geq 2$ and let $\left\{a_{1}, \ldots, a_{g}\right.$, $\left.b_{1}, \ldots, b_{g}\right\}$ be a canonical homology basis [13, III.1] giving the marking. Let $\left\{\theta_{1}, \ldots, \theta_{g}\right\}$ be the dual basis of holomorphic differentials on $M$, that is the basis characterized by the property that $\theta_{j}\left(a_{k}\right)=\delta_{j k}$. The $g \times g$ complex matrix $\Pi_{i j}:=\theta_{i}\left(b_{j}\right)$ is called the period matrix of the marked Riemann surface $M$. The period matrix defines a holomorphic map $\Pi: T_{g} \rightarrow \Sigma_{g}$ on the Teichmüller space $T_{g}$ with values in the Siegel space $\Sigma_{g}$ of symmetric complex matrices with positive definite imaginary part. Let $q \in Q_{g}$ be a holomorphic quadratic differential on $M$. Let $\mu_{q}:=|q| / q$ be the canonical Beltrami differential on $M$ associated to $q$, which represents the deformation of the complex structure of the Riemann surface $M_{q}$ in the direction determined by the Teichmüller flow at $\left(M_{q}, q\right)$. The equation

$$
\operatorname{det}\left(\frac{d \Pi}{d \mu_{q}}\right)=0
$$

defines a real analytic hypersurface $D_{g}$, of real codimension equal to 2 , of the Teichmüller space $Q_{g}$. In addition, $D_{g}$, is invariant under the action of the mapping class group $\Gamma_{g}$. The quotient $\mathcal{D}_{g}:=D_{g} / \Gamma_{g}$ is therefore a real analytic hypersurface of codimension 2 of the moduli space $\mathcal{M}_{g}$, which will be called the determinant locus. We remark that, since (4.1) is invariant under the multiplicative (holomorphic) action of the group $\mathbb{C}^{*}:=\mathbb{C} \backslash\{0\}$ on the space $Q_{g}$, the quotients $D_{g} / \mathbb{C}^{*}, \mathcal{D}_{g} / \mathbb{C}^{*}$ are real analytic hypersurfaces of $Q_{g}^{(1)} / \mathrm{SO}(2, \mathbb{R})$, $\mathcal{M}_{g}^{(1)} / \mathrm{SO}(2, \mathbb{R})$ respectively. Let $D_{\kappa}:=D_{g} \cap Q_{\kappa}, D_{\kappa}^{(1)}:=D_{g} \cap Q_{\kappa}^{(1)}, \mathcal{D}_{\kappa}:=$ $D_{\kappa} / \Gamma_{g}$ and $\mathcal{D}_{\kappa}^{(1)}:=D_{\kappa}^{(1)} / \Gamma_{g}$.

The answer to the question whether the lower bound established in Theorem 3.3 is strictly positive depends on the geometry of the determinant locus. In fact, the answer will be affirmative if the support of the probability measure $\mu$, ergodic with respect to the Teichmüller flow on $\mathcal{M}_{\kappa}^{(1)}$, is not contained in $\mathcal{D}_{\kappa}^{(1)}$. Let $q \in \mathcal{M}_{\kappa}^{(1)}$ and $\pi_{q}^{-}: L_{q}^{2}(M) \rightarrow \mathcal{M}_{q}^{-}$be the orthogonal projection onto the subspace of anti-meromorphic functions. Let

$$
1 \equiv \Lambda_{1}(q) \geq \Lambda_{2}(q) \geq \cdots \geq \Lambda_{g}(q) \geq 0
$$

be the eigenvalues of the non-negative hermitian form $H_{q}: \mathcal{M}_{q}^{+} \times \mathcal{M}_{q}^{+} \rightarrow \mathbb{R}$ defined by

$$
H_{q}\left(m_{1}^{+}, m_{2}^{+}\right):=\left(\pi_{q}^{-}\left(m_{1}^{+}\right), \pi_{q}^{-}\left(m_{2}^{+}\right)\right)_{q} .
$$

The functions $\Lambda_{1}, \ldots, \Lambda_{g}$ are continuous on $\mathcal{M}_{\kappa}^{(1)}$ and invariant under the action of $\mathrm{SO}(2, \mathbb{R})$.

LEMmA 4.1. The zero set $\left\{\Lambda_{g}=0\right\}=\mathcal{D}_{\kappa}^{(1)}$. 
Proof. Let $\left\{m_{1}^{+}, \ldots, m_{g}^{+}\right\}$be an orthonormal basis of $\mathcal{M}_{q}^{+}$. The (symmetric) matrix $B$ of the projection operator $\pi_{q}^{-}$, with respect to the basis $\left\{m_{1}^{+}, \ldots, m_{g}^{+}\right\} \subset \mathcal{M}_{q}^{+}$and $\left\{{\overline{m^{+}}}_{1}, \ldots,{\overline{m^{+}}}_{g}\right\} \subset \mathcal{M}_{q}^{-}$, and the matrix $H$ of the hermitian form $H_{q}$, with respect to the basis $\left\{m_{1}^{+}, \ldots, m_{g}^{+}\right\}$, are given by the following formulas:

$$
B_{i j}=B_{q}\left(m_{i}^{+}, m_{j}^{+}\right)=\left(m_{i}^{+},{\overline{m^{+}}}_{j}\right)_{q}, \quad H=B^{*} B=\bar{B} B .
$$

Let $\left\{a_{1}, \ldots, a_{g}, b_{1}, \ldots, b_{g}\right\}$ be a canonical homology basis and $\left\{\theta_{1}, \ldots, \theta_{g}\right\}$ be the dual basis of holomorphic differentials. The quotients $\phi_{i}^{+}:=\theta_{i} / q^{1 / 2}$ are meromorphic functions on $M$ with poles at $\Sigma_{q}$, which belong to the space $L_{q}^{2}(M)$. The system $\left\{\phi_{1}^{+}, \ldots, \phi_{g}^{+}\right\}$is a basis of the space $\mathcal{M}_{q}^{+}$. By Rauch's formula [28, Prop. A.3]:

$$
\frac{d \Pi_{i j}}{d \mu_{q}}=\int_{M} \theta_{i} \theta_{j} \mu_{q}=\int_{M} \phi_{i}^{+} \phi_{j}^{+} \omega_{q}=B_{q}\left(\phi_{i}^{+}, \phi_{j}^{+}\right) .
$$

Since $\left\{\phi_{1}^{+}, \ldots, \phi_{g}^{+}\right\}$is a basis of $\mathcal{M}_{q}^{+}$, there exists a non-singular $g \times g$ complex matrix $C=\left(c_{i j}\right)$ such that

$$
\phi_{i}^{+}=\sum_{j=1}^{g} c_{i j} m_{j}^{+}, \quad C C^{*}=\Im(\Pi) .
$$

In fact, by [13, III.2.3],

$$
\left(\phi_{i}^{+}, \phi_{j}^{+}\right)_{q}=\frac{\imath}{2} \int_{M} \theta_{i} \wedge \overline{\theta_{j}}=\frac{\imath}{2} \sum_{k=1}^{g} \theta_{i}\left(a_{k}\right) \overline{\theta_{j}}\left(b_{k}\right)-\theta_{i}\left(b_{k}\right) \overline{\theta_{j}}\left(a_{k}\right)=\Im\left(\Pi_{i j}\right) .
$$

By (4.4) and (4.5),

$$
\begin{aligned}
\left|\operatorname{det}\left(\frac{d \Pi}{d \mu_{q}}\right)\right| & =\left|\operatorname{det}\left(C B C^{t}\right)\right|=|\operatorname{det} C|^{2}|\operatorname{det} B| \\
& =\operatorname{det}(\Im(\Pi))(\operatorname{det} H)^{1 / 2}=\operatorname{det}(\Im(\Pi))\left(\Lambda_{1} \cdots \Lambda_{g}\right)^{1 / 2} .
\end{aligned}
$$

Since $\Im(\Pi)$ is positive definite, $\Lambda_{g}(q)=0$ if and only if $q \in \mathcal{D}_{\kappa}^{(1)}$.

The basic idea in order to prove that the lowest eigenvalue $\Lambda_{g}$ in (4.2) does not vanish identically is to study the determinant locus near the boundary of the moduli space. We briefly recall the methods introduced by Fay [15, III] and Masur [42] to analyze the behaviour of holomorphic and quadratic differentials near the boundary of the moduli space. We warn the reader that, in the author's opinion, all of the variational formulas contained in [15, III] are to some extent incorrect, as pointed out by A.Yamada in [71], where the correct formulas are proved in great detail (compare, for instance, the formulas (47), (53) in [15] with, respectively, (36), (36) and (56) in [71]. However, our 
argument does not require precise variational formulas (only the dominant term in the appropriate expansions), hence it does not depend on which version, Fay's or Yamada's, is considered to be the correct one.

The moduli space $R_{g}=T_{g} / \Gamma_{g}$ can be compactified by adding Riemann surfaces with nodes as a boundary. This compactification is explained in detail in [5] or [42]. A Riemann surface with nodes $M_{0}$ can be obtained by pinching a compact Riemann surface $M$ along disjoint curves $\gamma_{1}, \ldots, \gamma_{s}$ with $1 \leq s \leq$ $3 g-3$. A connected component of the complement of the set of nodes is called a part of $M_{0}$; it is a finite non-singular Riemann surface with punctures. It is possible to assume that no part of $M_{0}$ is a sphere with just one or two punctures. The pinching of each $\gamma_{i}$ produces two paired punctures on $M_{0}$, denoted by $p_{i}^{(1)}$, $p_{i}^{(2)}$. Let $z_{i}^{(1)}$ and $z_{i}^{(2)}$ be holomorphic coordinates parametrizing disjoint punctured disks $U_{i}^{(1)}=\left\{0<\left|z_{i}^{(1)}\right|<1\right\}$ and $U_{i}^{(2)}=\left\{0<\left|z_{i}^{(2)}\right|<1\right\}$ in a neighbourhood of $p_{i}^{(1)}$ and $p_{i}^{(2)}$ respectively. There are local coordinates $\left(\tau_{s+1}, \ldots, \tau_{3 g-3}\right)$ in a neighbourhood of $0 \in \mathbb{C}^{3 g-3-s}$, parametrizing a neighbourhood of $M_{0}$ in its moduli space, such that, for each $M_{\tau}$ near $M_{0}$, the functions $z_{i}^{(1)}, z_{i}^{(2)}$ are still holomorphic coordinates in a neighbourhood of the punctures. For each $\left(t_{1}, \ldots, t_{s}\right) \in \mathbb{C}^{s}$ in a neighbourhood of zero, consider the Riemann surface $M_{(t, \tau)}$ obtained by deleting all the neighbourhoods $\left\{0<\left|z_{i}^{(1)}\right| \leq\left|t_{i}\right|\right\}$ and $\left\{0<\left|z_{i}^{(2)}\right| \leq\left|t_{i}\right|\right\}$ from $M_{\tau}$ and gluing $z_{i}^{(1)}$ to $t_{i} / z_{i}^{(2)}$. If all $t_{i} \neq 0$, the surface $M_{(t, \tau)}$ is a compact Riemann surface of genus $g$. The parameters $(t, \tau)$ parametrize all Riemann surfaces in the compactified moduli space in a neighbourhood of $M_{0}$. Let $A_{t_{i}}:=\left\{\left|t_{i}\right|<\left|z_{i}^{(1)}\right|<1\right\}=\left\{\left|t_{i}\right|<\left|z_{i}^{(2)}\right|<1\right\} \subset M_{(t, \tau)}$ and let $\gamma_{t_{i}}$ be the curve defined by $\left|z_{i}^{(1)}\right|=\left|z_{i}^{(2)}\right|=\left|t_{i}\right|^{1 / 2}$. If $K$ is a compact subset of the complement of the set of the nodes in $M_{0}$, then for sufficiently small $(t, \tau)$ it is possible to regard $K$ as a compact subset of $M_{(t, \tau)}$ via the quasi-conformal map $\phi_{\tau}: M_{0} \rightarrow M_{\tau}$ and the natural inclusion of $M_{(t, \tau)} \backslash \cup\left\{\gamma_{t_{i}}\right\}$ in $M_{\tau}$.

There is a complex manifold $X$ of dimension $3 g-2$ and a proper holomorphic map $\pi: X \rightarrow \mathbb{C}^{s} \times \mathbb{C}^{3 g-3-s}$ such that the fiber over $(t, \tau)$ is $M_{(t, \tau)}$. Such a construction is explained by [15, III] or [42], we recall it here for the convenience of the reader. Let $\tilde{W}:=\cup M_{\tau}$ and $\tilde{Y}:=\tilde{W} \backslash \cup \phi_{\tau}\left(U_{i}^{(1)} \cup U_{i}^{(2)}\right)$, where the union is taken over $i=1, \ldots, s, \tau \in U$ and $U \subset \mathbb{C}^{3 g-3-s}$ is a neighbourhood of the origin. Let

$$
W:=\tilde{Y} \times D^{s} \cup \bigcup\left\{(p, t) \in \phi_{\tau}\left(U_{i}^{(k)}\right) \times D^{s}|| z_{i}^{(k)}(p)|>| t_{i} \mid\right\},
$$

where $D \subset \mathbb{C}$ denotes the open unit disk. Let then $V_{i} \subset \mathbb{C}^{3}$ be the non-singular surface given by the equation $X_{i} Y_{i}=t_{i}$ and let

$$
X:=W \cup\left(V_{1} \times D^{s-1} \times U\right) \cup \cdots \cup\left(V_{s} \times D^{s-1} \times U\right),
$$


where in the overlap $(p, t)$ is identified with

$$
\begin{aligned}
&\left(z_{i}^{(1)}(p), t_{i} / z_{i}^{(1)}(p), t_{i}, t_{1}, \ldots, t_{i-1}, t_{i+1}, \ldots, t_{s}\right) \\
& \text { or } \quad\left(t_{i} / z_{i}^{(2)}(p), z_{i}^{(2)}(p), t_{i}, t_{1}, \ldots, t_{i-1}, t_{i+1}, \ldots, t_{s}\right),
\end{aligned}
$$

according to whether $(p, t) \in W \cap \phi_{\tau}\left(U_{i}^{(1)}\right) \times D^{s}$ or $(p, t) \in W \cap \phi_{\tau}\left(U_{i}^{(2)}\right) \times D^{s}$. It can be proved that $X$ is a complex manifold. Local coordinates at a point $\left(\phi_{\tau}(p), t\right) \in W$ are given by $\left(\phi_{\tau}(z), t, \tau\right)$, where $z$ is a holomorphic coordinate for $M_{0}$ in a neighbourhood of $p$. The natural projection $\pi: X \rightarrow D^{s} \times U$ has fiber $M_{(t, \tau)}$. It is a standard procedure to introduce the coordinates

$$
x_{i}:=\frac{z_{i}^{(1)}+z_{i}^{(2)}}{2}, \quad y_{i}:=\frac{z_{i}^{(1)}-z_{i}^{(2)}}{2}
$$

on $V_{i}$. Then $y_{i}=\left(x_{i}^{2}-t_{i}\right)^{1 / 2}$ represents $A_{t_{i}} \cap V_{i}$ as a branched double covering of a neighbourhood of $x_{i}=0$ with branch points at $x_{i}= \pm t_{i}^{1 / 2}$. At $t_{i}=0$ the fiber crosses itself at the node corresponding to the pinching of the curve $\gamma_{i}$, given by the equations $x_{i}=y_{i}=t_{i}=0$, and the normalization of $A_{0}$ is $U_{i}^{(1)} \cup U_{i}^{(2)}$. The $x_{i}$ are called the pinching coordinates for the corresponding nodes. Holomorphic (abelian) differentials on $M_{(t, \tau)}$ are customarily written in terms of these coordinates. A regular d-differential on a finite Riemann surface $M$ with punctures is by definition a meromorphic form of type $(d, 0)$ on each part of $M$, holomorphic in the complement of the punctures, with poles of order at most $d$ at the punctures and equal (opposite) residues at paired punctures if $d$ is even (odd). The residue of a regular $d$-differential $q$ at a point $p \in M$, denoted by $\operatorname{Res}_{q}(p)$, is the residue at $z=0$ (in the standard sense) of the abelian differential $z^{d-1} q(z) d z$, where $z$ is a holomorphic coordinate such that $z(p)=0$ and $q=q(z) d z^{2}[42, \S 4]$. Only the cases $d=1$ and $d=2$ of abelian and quadratic differentials are relevant in this paper. Any regular 1-differential $u(t, \tau)$ on $M_{(t, \tau)}$, holomorphic on $A_{t_{i}}$ and as a function on the total space $X$, can be written in terms of the pinching coordinate $x_{i}$ as follows $[15, \mathrm{III}],[42,4.1]$ :

$$
u(t, \tau)=\left[a\left(t, \tau, x_{i}\right)+b\left(t, \tau, x_{i}\right) /\left(x_{i}^{2}-t_{i}\right)^{1 / 2}\right] d x_{i},
$$

where $a, b$ are holomorphic functions. A direct computations shows that, under the change of variables given by (4.9), we have, with respect to the coordinate $z^{(k)}$ on $A_{t_{i}}, k=1,2$ :

$$
u(t, \tau)=\left[f^{(k)}\left(t, \tau, z^{(1)}+z^{(2)}\right)+\frac{g^{(k)}\left(t, \tau, z^{(1)}+z^{(2)}\right)}{z^{(k)}}\right] d z^{(k)},
$$

where $f^{(k)}, g^{(k)}$ are holomorphic functions. 
There is a normalized basis $\left\{\theta_{1}(t, \tau), \ldots, \theta_{g}(t, \tau)\right\}$ of regular 1-differentials on $M_{(t, \tau)}$, holomorphic on $X$ [15, III], [42, Prop. 4.1], [71, Corollaries 1,4,5], with respect to a canonical homology basis

$$
\left\{a_{1}(t, \tau), \ldots, a_{g}(t, \tau), b_{1}(t, \tau), \ldots, b_{g}(t, \tau)\right\}
$$

Let $\Pi_{(t, \tau)}$ be the corresponding period matrix, which is well-defined (finite) if all $t_{i} \neq 0$. Variational formulas for the period matrix as $t_{i} \rightarrow 0$ were obtained in $[15, \mathrm{III}]$ and (corrected) in [71]. Let $q(t, \tau) \in Q_{g}$ be a holomorphic quadratic differential on $M_{(t, \tau)}$, holomorphic as a function on $X$, which converges in the projective bundle $Q_{g} / \mathbb{R}^{*}$ as $t_{i} \rightarrow 0, i \in J \subset\{1, \ldots, s\}$, to a regular quadratic differential having poles of order two at all the punctures and different from zero on every part of the corresponding pinched Riemann surface. Let $\mu_{(t, \tau)}:=$ $|q(t, \tau)| / q(t, \tau)$ be the related family of Beltrami differentials. We prove a result concerning the limit behaviour of the derivative of the period matrix $\Pi_{(t, \tau)}$ in the direction of the Beltrami differential $\mu_{(t, \tau)}$ at the boundary of the moduli space. Let $M_{\tau}(t)$ be the image of the natural inclusion of $M_{(t, \tau)} \backslash \cup\left\{\gamma_{t_{i}}\right\}$ in $M_{\tau}$. The surface $M_{\tau}(t)$ is an open subset of $M_{\tau}$ which can be described as follows:

$$
M_{\tau}(t)=M_{\tau} \backslash \bigcup_{i=1}^{s}\left(\left\{\left|z_{i}^{(1)}\right| \leq\left|t_{i}\right|^{1 / 2}\right\} \cup\left\{\left|z_{i}^{(2)}\right| \leq\left|t_{i}\right|^{1 / 2}\right\}\right) .
$$

Lemma 4.2. As $t_{i} \rightarrow 0$, for all $i \in J \subset\{1, \ldots, s\}$,

$$
\frac{d \Pi_{(t, \tau)}^{i j}}{d \mu_{(t, \tau)}}-\int_{M_{\tau}(t)} \theta_{i}\left(t^{\prime}, \tau\right) \theta_{j}\left(t^{\prime}, \tau\right) \mu_{\left(t^{\prime}, \tau\right)} \rightarrow 0,
$$

where $t_{i}^{\prime}=0$ if $i \in J, t_{i}^{\prime}=t_{i} \neq 0$ if $i \notin J$.

Proof. By Rauch's formula (4.4), the derivative of the period matrix can be written as an integral over $M_{\tau}(t)$, identified to the complement of the finite set of curves $\gamma_{t_{i}}$ in $M_{(t, \tau)}$. In addition, since the integrand is uniformly bounded and converges on compact sets contained in the complement of the punctures by the variational formulas of $[15, \mathrm{III}]$ or [71], it suffices to prove convergence on each annulus

$$
R_{t_{i}}^{(k)}:=\left\{\left|t_{i}\right|^{1 / 2}<\left|z_{i}^{(k)}\right|<1\right\} \subset A_{t_{i}},
$$

for all $i \in J, k=1,2$.

Let $R_{t}:=\left\{|t|^{1 / 2}<|z|<1\right\}$ one of the annuli in (4.13). We should estimate, as $t \rightarrow 0$ in $\mathbb{C}$, an expression of the form

$$
\int_{R_{t}}\left(\theta_{i}(t, z) \theta_{j}(t, z) \frac{\left|q_{t}(z)\right|}{q_{t}(z)}-\theta_{i}(0, z) \theta_{j}(0, z) \frac{\left|q_{0}(z)\right|}{q_{0}(z)}\right) d z \wedge d \bar{z},
$$


where $\theta_{i}(t, z)$ are meromorphic functions with at most a simple pole at $z=0$ given by $\left(4.10^{\prime}\right)$, that is

$$
\theta_{i}(t, z)=f_{i}(t, z+t / z)+\frac{g_{i}(t, z+t / z)}{z}=\frac{F_{i}(t, z+t / z)}{z},
$$

with $f_{i}, g_{i}$, hence $F_{i}$, holomorphic functions, and $q_{t}(z)$ is a meromorphic function with a double pole at $z=0$, which can be written as $[42,5.2-5.3]$ :

$$
q_{t}(z)=\frac{a(t, z+t / z)}{z}+\frac{b(t, z+t / z)}{z^{2}}=\frac{A(t, z+t / z)}{z^{2}},
$$

with $a, b$, hence $A$, holomorphic functions and $A(0,0) \neq 0$. We split the difference in (4.14) into the following three terms:

$$
\begin{aligned}
& \text { (I) } \int_{R_{t}}\left(\theta_{i}(t, z)-\theta_{i}(0, z)\right) \theta_{j}(t, z) \frac{\left|q_{t}(z)\right|}{q_{t}(z)} d z \wedge d \bar{z}, \\
& \text { (II) } \int_{R_{t}}\left(\theta_{j}(t, z)-\theta_{j}(0, z)\right) \theta_{i}(0, z) \frac{\left|q_{t}(z)\right|}{q_{t}(z)} d z \wedge d \bar{z}, \\
& \text { (III) } \int_{R_{t}} \theta_{i}(0, z) \theta_{j}(0, z)\left(\frac{\left|q_{t}(z)\right|}{q_{t}(z)}-\frac{\left|q_{0}(z)\right|}{q_{0}(z)}\right) d z \wedge d \bar{z} .
\end{aligned}
$$

By (4.15), it follows that, since $t / z \rightarrow 0$ if $z \in R_{t}$ as $t \rightarrow 0$, there is a constant $C_{0}>0$ such that

$$
\left|\theta_{i}(t, z)-\theta_{i}(0, z)\right| \leq C_{0} \frac{|t|}{|z|^{2}} \quad, \quad\left|\theta_{i}(t, z)\right| \leq C_{0} \frac{1}{|z|} .
$$

By Hölder inequality and (4.17), we obtain:

$$
\begin{aligned}
& |(\mathrm{I})| \leq\left\|\theta_{i}(t, z)-\theta_{i}(0, z)\right\|_{L^{2}\left(R_{t}\right)}\left\|\theta_{j}(t, z)\right\|_{L^{2}\left(R_{t}\right)} \leq C_{1}(-|t| \log |t|)^{1 / 2} \\
& |(\mathrm{II})| \leq\left\|\theta_{j}(t, z)-\theta_{j}(0, z)\right\|_{L^{2}\left(R_{t}\right)}\left\|\theta_{i}(0, z)\right\|_{L^{2}\left(R_{t}\right)} \leq C_{1}(-|t| \log |t|)^{1 / 2}
\end{aligned}
$$

In order to estimate the third term in (4.16), we proceed as follows. By $\left(4.15^{\prime}\right)$, since $A(0,0) \neq 0$, there exists $r>0$ such that $A(t, z) \neq 0$, hence $|A(t, z)| / A(t, z)$ is a real analytic function, in the polydisk $\{|t|<r$, $|z|<r\} \subset \mathbb{C}^{2}$. There exists therefore a constant $C_{2}>0$ such that, in the annulus $\left\{|t|^{1 / 2}<|z|<r / 2\right\}$, we have:

$$
\left|\frac{\left|q_{t}(z)\right|}{q_{t}(z)}-\frac{\left|q_{0}(z)\right|}{q_{0}(z)}\right|=\left|\frac{|A(t, z+t / z)|}{A(t, z+t / z)}-\frac{|A(0, z)|}{A(0, z)}\right| \leq C_{2} \frac{|t|}{|z|} \leq C_{2}|t|^{1 / 2} .
$$

It follows that

$$
\begin{aligned}
|(\mathrm{III})| & \leq-C_{3}|t|^{1 / 2} \log |t| \\
& +\left|\int_{|z| \geq r / 2} \theta_{i}(0, z) \theta_{j}(0, z)\left(\frac{\left|q_{t}(z)\right|}{q_{t}(z)}-\frac{\left|q_{0}(z)\right|}{q_{0}(z)}\right) d z \wedge d \bar{z}\right|,
\end{aligned}
$$

where the integral on the right-hand side converges to zero as $t \rightarrow 0$ by the dominated convergence theorem. By $(4.18)$ and $\left(4.19^{\prime}\right)$ the proof is concluded. 
By Lemma 4.2 and the variational formulas for the period matrix proved in $[15, \mathrm{III}],[71]$, it is possible to estimate the determinant of the derivative of the period matrix and, by the identity (4.6), the determinant of the hermitian form $H_{q}$ close to the boundary of the moduli space, by computing them at the boundary. Let in particular $\mathcal{S}_{g}$ be the component of the boundary consisting of regular quadratic differentials on the Riemann sphere with $2 g$ paired punctures, having poles of order 2 at all punctures.

Lemma 4.2'. Let $q_{0} \in \mathcal{S}_{g}$. Let $M_{0}$ be the corresponding Riemann sphere with the $2 g$ paired punctures $p_{i}^{(1)}, p_{i}^{(2)}, i=1, \ldots, g$. Let $t \in \mathbb{C}^{g}$ be such that $t_{i} \neq 0$ for all $i=1, \ldots, g$ and $M_{0}(t) \subset M_{0}$ be the open subset defined as in (4.11). Let $\left\{\theta_{1}(0), \ldots, \theta_{g}(0)\right\}$ be the basis of the space of regular 1-differentials on $M_{0}$, dual to the canonical homology basis $\left\{a_{1}, \ldots, a_{g}, b_{1}, \ldots b_{g}\right\}$, where $a_{i}$ is represented by the (oriented) boundary of a disk centered at $p_{i}^{(1)}$ (not containing other punctures) and $b_{i}$ by a path joining $p_{i}^{(1)}$ to $p_{i}^{(2)}$. Then

$$
\int_{M_{0}(t)} \theta_{i}(0) \theta_{j}(0) \frac{\left|q_{0}\right|}{q_{0}}-\frac{1}{2 \pi} \frac{\left|\operatorname{Res}_{q_{0}}\left(p_{i}^{(1)}\right)\right|}{\operatorname{Res}_{q_{0}}\left(p_{i}^{(1)}\right)} \delta_{i j} \log \left|t_{i}\right|
$$

is bounded as $t \rightarrow 0$, for all $i, j \in\{1, \ldots, g\}$.

Proof. The normalized basis $\left\{\theta_{1}(0), \ldots, \theta_{g}(0)\right\}$ on the punctured Riemann sphere $M_{0}$ can be explicitly written:

$$
\theta_{i}(0)=\frac{p_{i}^{(1)}-p_{i}^{(2)}}{2 \pi \imath} \frac{d z}{\left(z-p_{i}^{(1)}\right)\left(z-p_{i}^{(2)}\right)} .
$$

In fact, (4.21) gives the only basis of regular 1-differential $\left\{\theta_{1}, \ldots, \theta_{g}\right\}$ satisfying $\theta_{i}\left(a_{j}\right)=\delta_{i j}$. Let $R_{i}^{(k)}(t):=\left\{\left|t_{i}\right|^{1 / 2}<\left|z-p_{i}^{(k)}\right|<r\right\}$, where $r>0$ has been chosen so that all the annuli $R_{i}^{(k)}(t)$ are disjoint and $q_{0}$ has no zeroes inside the closed disks $D_{r}\left(p_{i}^{(k)}\right)$ of radius $r$ centered at the punctures. Let

$$
q_{0}=\frac{A_{i}^{(k)}(z)}{\left(z-p_{i}^{(k)}\right)^{2}} d z^{2}
$$

on the disk $D_{r}\left(p_{i}^{(k)}\right)$. Since $q_{0}$ is a regular quadratic differential with poles of order 2 at all punctures, it follows that

$$
A_{i}^{(1)}\left(p_{i}^{(1)}\right)=\operatorname{Res}_{q_{0}}\left(p_{i}^{(1)}\right)=A_{i}^{(2)}\left(p_{i}^{(2)}\right)=\operatorname{Res}_{q_{0}}\left(p_{i}^{(2)}\right) \neq 0 .
$$

We have

$$
\int_{R_{i}^{(k)}(t)} \theta_{i}(0)^{2} \frac{\left|q_{0}\right|}{q_{0}}=\int_{R_{i}^{(k)}(t)} \frac{F_{i}^{(k)}(z)}{\left|z-p_{i}^{(k)}\right|^{2}} d z \wedge d \bar{z},
$$


where the function $F_{i}^{(k)}$ is given on the disk $D_{r}\left(p_{i}^{(k)}\right)$ by the formula:

$$
F_{i}^{(k)}(z):=\frac{\left(p_{i}^{(1)}-p_{i}^{(2)}\right)^{2}}{8 \pi^{2} \imath\left(z-p_{i}^{(h)}\right)^{2}} \frac{\left|A_{i}^{(k)}(z)\right|}{A_{i}^{(k)}(z)},
$$

where $k, h \in\{1,2\}$ and $k \neq h$. Hence $F_{i}^{(k)}$ is real analytic on the disk $D_{r}\left(p_{i}^{(k)}\right)$ and, by Taylor series expansion at $p_{i}^{(k)}$, we obtain that

$$
\int_{R_{i}^{(k)}(t)} \frac{F_{i}^{(k)}(z)}{\left|z-p_{i}^{(k)}\right|^{2}} d z \wedge d \bar{z}-F_{i}^{(k)}\left(p_{i}^{(k)}\right) \int_{R_{i}^{(k)}(t)} \frac{d z \wedge d \bar{z}}{\left|z-p_{i}^{(k)}\right|^{2}}
$$

is bounded as $t \rightarrow 0$. Similarly, if $i \neq j$, we obtain

$$
\int_{R_{i}^{(k)}(t)} \theta_{i}(0) \theta_{j}(0) \frac{\left|q_{0}\right|}{q_{0}}=\int_{R_{i}^{(k)}(t)} \frac{F_{i j}^{(k)}(z)}{\left(\overline{z-p_{i}^{(k)}}\right)} d z \wedge d \bar{z},
$$

where the function $F_{i j}^{(k)}$ is given on the disk $D_{r}\left(p_{i}^{(k)}\right)$ by the formula:

$$
F_{i j}^{(k)}(z):=\frac{\left(p_{i}^{(1)}-p_{i}^{(2)}\right)\left(p_{j}^{(1)}-p_{j}^{(2)}\right)}{8 \pi^{2} \imath\left(z-p_{i}^{(h)}\right)\left(z-p_{j}^{(1)}\right)\left(z-p_{j}^{(2)}\right)} \frac{\left|A_{i}^{(k)}(z)\right|}{A_{i}^{(k)}(z)}
$$

where, as above, $k, h \in\{1,2\}$ and $k \neq h$. Since $F_{i j}^{(k)}$ is real analytic, hence bounded, on the disk $D_{r}\left(p_{i}^{(k)}\right)$, the integral in (4.25) is bounded as $t \rightarrow 0$.

Let $R_{i j}(t):=R_{i}^{(1)}(t) \cup R_{i}^{(2)}(t) \cup R_{j}^{(1)}(t) \cup R_{j}^{(2)}(t)$. The integral

$$
\int_{M_{0}(t) \backslash R_{i j}(t)} \theta_{i}(0) \theta_{j}(0) \frac{\left|q_{0}\right|}{q_{0}}
$$

is bounded as $t \rightarrow 0$, for all $i, j \in\{1, \ldots, g\}$, since the integrand is uniformly bounded on $M_{0}(t) \backslash R_{i j}(t)$. Our statement follows, by (4.23), by the boundedness of the difference in (4.24) and of the integrals in (4.25), (4.26), by explicit computation of the term on the right of (4.24) and by the formulas $\left(4.22^{\prime}\right)$ on the residues of $q_{0}$.

Definition 4.3. A measured foliation $\mathcal{F}$ on a compact orientable surface $M$ of genus $g \geq 2$ is said to be periodic if all its regular leaves are closed (compact) curves. A periodic measured foliation $\mathcal{F}$ will be called Lagrangian if the subspace $\mathcal{L}(\mathcal{F}) \subset H_{1}(M, \mathbb{R})$, generated by the homology classes of the regular leaves of $\mathcal{F}$, is a Lagrangian subspace of the homology vector space $H_{1}(M, \mathbb{R})$, endowed with the symplectic structure given by the intersection form. A periodic measured foliation $\mathcal{F}$ is Lagrangian if and only if it has $g \geq 2$ distinct regular leaves $\gamma_{1}, \ldots, \gamma_{g}$ such that $\widehat{M}:=M \backslash \cup\left\{\gamma_{1}, \ldots, \gamma_{g}\right\}$ is homeomorphic to a sphere minus $2 g$ (paired) disjoint disks. 
Let $\mathcal{L}_{\kappa}^{ \pm}$be the set of holomorphic quadratic differentials $q \in Q_{\kappa}$ such that the foliation $\mathcal{F}_{ \pm q}$ is Lagrangian. Let $\Lambda(M, \mathbb{R})$ be the Grassmannian of Lagrangian subspaces of the symplectic vector space $H_{1}(M, \mathbb{R})$. Let $\Lambda \in$ $\Lambda(M, \mathbb{R})$ and let

$$
\mathcal{L}_{\kappa, \Lambda}^{ \pm}:=\left\{q \in \mathcal{L}_{\kappa}^{ \pm} \mid \mathcal{L}\left(\mathcal{F}_{ \pm q}\right) \cap \Lambda=\{0\}\right\}
$$

The final key step consists of the following crucial result:

Lemma 4.4. The set $\mathcal{L}_{\kappa, \Lambda}^{+} \subset \mathcal{L}_{\kappa}^{+}\left[\mathcal{L}_{\kappa, \Lambda}^{-} \subset \mathcal{L}_{\kappa}^{-}\right]$of holomorphic quadratic differentials $q \in Q_{\kappa}$ such that the horizontal foliation $\mathcal{F}_{q}$ [the vertical foliation $\left.\mathcal{F}_{-q}\right]$ is Lagrangian and $\mathcal{L}\left(\mathcal{F}_{q}\right) \cap \Lambda=\{0\}\left[\mathcal{L}\left(\mathcal{F}_{-q}\right) \cap \Lambda=\{0\}\right]$ is dense in $Q_{\kappa}$.

Proof. Let $\kappa:=\left(k_{1}, \ldots, k_{\sigma}\right)$, where all $k_{i}$ are even and $\sum k_{i}=4 g-4$. Let $\mathcal{F}_{\kappa}(M)$ be the set of all isotopy equivalence classes of orientable measured foliations $\mathcal{F}$ on $M$ with canonical saddle-like singularities of multiplicities $\left(k_{1}, \ldots, k_{\sigma}\right)$ at a finite set $\Sigma_{\mathcal{F}}:=\left\{p_{1}, \ldots, p_{\sigma}\right\}$. Let $\mathcal{F}_{\kappa}^{\prime}(M) \subset \mathcal{F}_{\kappa}(M)$ be the subset given by the measured foliations $\mathcal{F}$ such that there exists $q \in Q_{\kappa}$ with $\mathcal{F}_{q}=\mathcal{F}$ (or $\left.\mathcal{F}_{-q}=\mathcal{F}\right)$. The set $\mathcal{F}_{\kappa}^{\prime}(M)$ is open in $\mathcal{F}_{\kappa}(M)$ and the map $Q_{\kappa} \rightarrow \mathcal{F}_{\kappa}^{\prime}(M) \times \mathcal{F}_{\kappa}^{\prime}(M)$, given by $q \rightarrow\left(\mathcal{F}_{q}, \mathcal{F}_{-q}\right)$, is open. By A.Katok's local classification theorem, announced in [29, Th. 3] and proved in [32, Th. 14.7.4] or [47, Th. 7.11.7], the space $\mathcal{F}_{\kappa}(M)$ is locally modeled on $H^{1}\left(M, \Sigma_{\kappa} ; \mathbb{R}\right)$, while the space $Q_{\kappa}$ is locally modeled on $H^{1}\left(M, \Sigma_{\kappa} ; \mathbb{C}\right.$ ) (see $\S 1$ ). Let $\Lambda \in \Lambda(M, \mathbb{R}$ ). We claim that the set of Lagrangian foliations $\mathcal{F} \in \mathcal{F}_{\kappa}(M)$ such that $\mathcal{L}(\mathcal{F}) \cap \Lambda$ $=\{0\}$ is dense in $\mathcal{F}_{\kappa}(M)$. The proof of the claim will conclude the argument.

Let $\mathcal{F}:=\left\{\eta_{\mathcal{F}}=0\right\} \in \mathcal{F}_{\kappa}(M)$ and let $\Sigma_{\mathcal{F}} \subset M$ denote its (finite) singularity set. If the cohomology class $\left[\eta_{\mathcal{F}}\right] \in \mathbb{R} \cdot H^{1}\left(M, \Sigma_{\mathcal{F}} ; \mathbb{Z}\right)$, hence, in particular, if $\left[\eta_{\mathcal{F}}\right] \in H^{1}\left(M, \Sigma_{\mathcal{F}} ; \mathbb{Q}\right)$, then $\mathcal{F}$ is periodic. In fact, in that case, the set of $\mathcal{F}$-length of isotopy classes of simple closed curves is discrete. Hence, all the regular leaves are closed by the Poincaré recurrence theorem. It follows that the periodic measured foliations are dense in $\mathcal{F}_{\kappa}(M)$. If $\mathcal{F}$ is periodic, the surface can be decomposed, by cutting along the singular leaves, into a finite union of cylindrical components whose number is at most $3 g-3$. Let $d:=\operatorname{dim} \mathcal{L}(\mathcal{F}) \in\{1, \ldots, g\}$, where $\mathcal{L}(\mathcal{F}) \subset H_{1}(M, \mathbb{Z})$ is given by Definition 4.3. If $d=g$, then $\mathcal{F}$ is a Lagrangian measured foliation.

Let $P: H_{1}(M, \mathbb{R}) \rightarrow H^{1}(M, \mathbb{R})$ be the (symplectic) map given by the Poincaré duality. We claim that, if $\mathcal{F}$ is periodic, then $P^{-1}\left[\eta_{\mathcal{F}}\right] \in \mathcal{L}(\mathcal{F})$. More precisely, if $\left\{a_{1}, \ldots, a_{s}\right\}$ are the oriented waist curves of the cylinders $\left\{A_{1}, \ldots, A_{s}\right\}$ of $\mathcal{F}$, which are respectively of heights $\left\{h_{1}, \ldots, h_{s}\right\}$, then

$$
P^{-1}\left[\eta_{\mathcal{F}}\right]=\sum_{i=1}^{s} h_{i}\left[a_{i}\right] \in H_{1}(M, \mathbb{R}) .
$$


In fact, if $\gamma \subset M$ is any simple oriented closed curve, then $\gamma \cap A_{i}$ is homologous to $\left(\left[a_{i}\right] \cap[\gamma]\right) \cdot v_{i}$ relative to $\partial A_{i}$, where $v_{i}$ is a positively oriented vertical segment joining the ends of $A_{i}$. Hence (4.28) follows.

Let $\Lambda \in \Lambda(M, \mathbb{R})$ and let $\left\{a_{1}, \ldots, a_{k}\right\}$ be a maximal system of regular leaves of $\mathcal{F}$ such that the system of homology classes $\left\{\left[a_{1}\right], \ldots,\left[a_{k}\right]\right\}$ is linearly independent in $H_{1}(M, \mathbb{R})$ and the isotropic subspace $I\left(a_{1}, \ldots, a_{k}\right) \subset$ $H_{1}(M, \mathbb{R})$, generated by $\left\{\left[a_{1}\right], \ldots,\left[a_{k}\right]\right\}$, is transverse to $\Lambda$. If $k=g$, then $\mathcal{F}$ is Lagrangian and $\mathcal{L}(\mathcal{F}) \cap \Lambda=\{0\}$. If $k<g$, then there exists a primitive homology class $h \in H_{1}(M, \mathbb{Z})$ such that $h \cap\left[a_{1}\right]=\cdots=h \cap\left[a_{k}\right]=0$ and $h \notin I\left(a_{1}, \ldots, a_{k}\right) \oplus \Lambda$. In fact, since $\Lambda$ is Lagrangian, the dimension of the subspace $\left\{h \in I\left(a_{1}, \ldots, a_{k}\right) \oplus \Lambda \mid h \cap\left[a_{1}\right]=\cdots=h \cap\left[a_{k}\right]=0\right\}$ is equal to $g$. On the other hand, the dimension over $\mathbb{Q}$ of the subspace $\left\{h \in H_{1}(M, \mathbb{Q}) \mid h \cap\left[a_{1}\right]=\cdots=h \cap\left[a_{k}\right]=0\right\}$ is equal to $2 g-k$. Since $2 g-k>g$, there exists $\widehat{h} \in H_{1}(M, \mathbb{Q})$ such that $\widehat{h} \notin I\left(a_{1}, \ldots, a_{k}\right) \oplus \Lambda$ and $\widehat{h} \cap\left[a_{1}\right]=\cdots=\widehat{h} \cap\left[a_{k}\right]=0$. Let then $h \in H_{1}(M, \mathbb{Z})$ be the unique integer multiple of $\widehat{h}$ which is a primitive integer class.

Let $z=\sum_{i} n_{i} \gamma_{i}$ be an integer cycle on $M$, supported on a finite union of disjoint smooth simple closed curves $\left\{\gamma_{1}, \ldots, \gamma_{s}\right\}$ such that $[z]=h, \operatorname{supp}(z) \cap$ $a_{1}=\cdots=\operatorname{supp}(z) \cap a_{k}=\emptyset$ and $\operatorname{supp}(z) \cap \Sigma_{\mathcal{F}}=\emptyset$. Let $\mathcal{V}\left(\gamma_{i}\right) \subset \subset \mathcal{U}\left(\gamma_{i}\right)$ be open tubular neighbourhoods of $\gamma_{i}$ in $M$ such that, for all $i \neq j \in\{1, \ldots, s\}$,

$$
\begin{aligned}
& \overline{\mathcal{U}\left(\gamma_{i}\right)} \cap \overline{\mathcal{U}\left(\gamma_{j}\right)}=\emptyset, \\
& \overline{\mathcal{U}\left(\gamma_{i}\right)} \cap\left(a_{1} \cup \cdots \cup a_{k}\right)=\emptyset, \\
& \overline{\mathcal{U}\left(\gamma_{i}\right)} \subset M \backslash \Sigma_{\mathcal{F}} .
\end{aligned}
$$

Let $\mathcal{U}^{ \pm}\left(\gamma_{i}\right)$ be the two connected components of the open set $\mathcal{U}\left(\gamma_{i}\right) \backslash \gamma_{i}$ and let $\mathcal{V}^{ \pm}\left(\gamma_{i}\right):=\mathcal{V}\left(\gamma_{i}\right) \cap \mathcal{U}^{ \pm}\left(\gamma_{i}\right)$. Let $\phi_{i}: M \rightarrow \mathbb{R}$ be a function, smooth on $M \backslash \gamma_{i}$, with the following properties:

$$
\begin{array}{ll}
\phi_{i}(p)=0, & p \in \mathcal{U}^{-}\left(\gamma_{i}\right) \cup M \backslash \mathcal{U}^{+}\left(\gamma_{i}\right), \\
\phi_{i}(p)=1, & p \in \overline{\mathcal{V}^{+}\left(\gamma_{i}\right)} .
\end{array}
$$

Let $r \in \mathbb{Q} \backslash\{0\}$. The smooth 1-form $\eta_{r}:=\eta_{\mathcal{F}}+r \sum_{i} n_{i} d \phi_{i}$ is closed and $\eta_{r} \rightarrow \eta_{\mathcal{F}}$, as $r \rightarrow 0$, in the space of smooth 1 -forms on $M$. Since $d \phi_{i}=0$ on $M \backslash \mathcal{U}\left(\gamma_{i}\right), \eta_{r} \equiv \eta_{\mathcal{F}}$ in a neighbourhood of $\Sigma_{\mathcal{F}}$, hence, if $r \neq 0$ is sufficiently small, $\eta_{r}(p)=0$ if and only if $p \in \Sigma_{\mathcal{F}}$, and the foliation $\mathcal{F}_{r}:=\left\{\eta_{r}=0\right\} \in$ $\mathcal{F}_{\kappa}(M)$. Since $r \in \mathbb{Q}$, the fundamental class $\left[\eta_{r}\right] \in H^{1}\left(M, \Sigma_{\mathcal{F}} ; \mathbb{Q}\right)$, hence $\mathcal{F}_{r}$ is periodic. The simple closed curves $a_{1}, \ldots, a_{k}$ are regular leaves of $\mathcal{F}_{r}$. In fact, $d \phi_{i}=0$ on $M \backslash \mathcal{U}\left(\gamma_{i}\right)$ and $\cup\left\{a_{1}, \ldots, a_{k}\right\} \subset M \backslash \cup \overline{\mathcal{U}}\left(\gamma_{i}\right)$, hence $\eta_{r} \equiv \eta_{\mathcal{F}}$ in a neighbourhood of $\cup\left\{a_{1}, \ldots, a_{k}\right\}$. Let $I_{r} \subset H_{1}(M, \mathbb{R})$ be the maximal isotropic subspace, generated by the regular leaves of $\mathcal{F}_{r}$, such that $I\left(a_{1}, \ldots, a_{k}\right) \subset I_{r}$ and $I_{r} \cap \Lambda=\{0\}$. We claim that $I_{r} \neq I\left(a_{1}, \ldots, a_{k}\right)$. If the latter claim is false, 
$\mathcal{L}\left(\mathcal{F}_{r}\right) \subset I\left(a_{1}, \ldots, a_{k}\right) \oplus \Lambda$, hence, by $(4.28), P^{-1}\left[\eta_{r}\right] \in I\left(a_{1}, \ldots, a_{k}\right) \oplus \Lambda$, but, by $\left(4.29^{\prime}\right)$ and the definition of $\eta_{r}, P^{-1}\left[\eta_{r}\right]=P^{-1}\left[\eta_{\mathcal{F}}\right]+r h \notin I\left(a_{1}, \ldots, a_{k}\right) \oplus \Lambda$. In fact, by (4.28) and the definition of the system $\left\{a_{1}, \ldots, a_{k}\right\}, P^{-1}\left[\eta_{\mathcal{F}}\right] \in$ $I\left(a_{1}, \ldots, a_{k}\right) \oplus \Lambda$ and, by construction, $h \notin I\left(a_{1}, \ldots, a_{k}\right) \oplus \Lambda$.

By a finite iteration of the previous construction, we can show that the closure in $\mathcal{F}_{\kappa}(M)$ of the subset of all Lagrangian measured foliations $\mathcal{F}$, such that $\mathcal{L}(\mathcal{F}) \cap \Lambda=\{0\}$, contains the subset of all periodic measured foliations. Hence it coincides with the entire space $\mathcal{F}_{\kappa}(M)$. Our claim is therefore proved.

Lemma $4.4^{\prime}$. Every connected component $\mathcal{C}_{\kappa}$ of any stratum $\mathcal{M}_{\kappa}$ of the moduli space of quadratic differentials has a boundary point in $\mathcal{S}_{g}$ with $2 g$ (paired) real strictly positive residues.

Proof. Let $\left\{\gamma_{1}, \ldots, \gamma_{g}\right\}$ be a system disjoint simple closed curves on $M$ with the property that $\widehat{M}:=M \backslash \cup\left\{\gamma_{1}, \ldots, \gamma_{g}\right\}$ is homeomorphic to a sphere minus $2 g$ disjoint disks. Let $\mathcal{V}_{\kappa}\left(\gamma_{i}\right)$ be the open subset of quadratic differentials $q \in Q_{\kappa}$ such that the vertical foliation $\mathcal{F}_{q}=\left\{\Re\left(q^{1 / 2}\right)=0\right\}$ has a closed regular leaf $\gamma_{i}(q)$ isotopic to $\gamma_{i}$ and let $\mathcal{V}_{\kappa}\left(\gamma_{1}, \ldots, \gamma_{g}\right):=\cap \mathcal{V}_{\kappa}\left(\gamma_{i}\right), i=1, \ldots, g$. By Lemma 4.4, since $\mathcal{C}_{\kappa} \subset \mathcal{M}_{\kappa}$ is open, the system $\left\{\gamma_{1}, \ldots, \gamma_{g}\right\}$ can be chosen with the additional property that the pull-back of $\mathcal{C}_{\kappa}$ to the Teichmüller space $Q_{\kappa}$ has non-empty intersection with $\mathcal{V}_{\kappa}\left(\gamma_{1}, \ldots, \gamma_{g}\right)$. We then construct a continuous deformation $\Phi:(0,1]^{g} \times \mathcal{V}_{\kappa}\left(\gamma_{1}, \ldots, \gamma_{g}\right) \rightarrow \mathcal{V}_{\kappa}\left(\gamma_{1}, \ldots, \gamma_{g}\right)$ having the properties listed below. Such a construction will conclude the proof. Let $q_{t}:=\Phi_{t}(q)$, $t=\left(t_{1}, \ldots, t_{g}\right) \in(0,1]^{g}$. Then

(1) If $t_{i}=1$ for all $i=1, \ldots, g$, then $q_{t}=q$.

(2) If $t_{i} \rightarrow 0$ for all $i=1, \ldots, g$, then the Riemann surface carrying $q_{t}$ converges to a Riemann surface with nodes, pinched along the curves $\gamma_{1}, \ldots, \gamma_{g}$, hence $q_{t}$ converges in the moduli space $\mathcal{M}_{g}$ to a quadratic differential $q_{0} \in \mathcal{S}_{g}$.

(3) The zero set of $q_{t}$ coincides with the zero set $\Sigma_{q}$ of $q$ for all $t \in(0,1]^{g}$ and the cohomology class $\left[\Im\left(q_{t}^{1 / 2}\right)\right]=\left[\Im\left(q^{1 / 2}\right)\right] \in H^{1}\left(M, \Sigma_{q}, \mathbb{R}\right)$.

We will construct $\Phi_{t}(q)$ as a composition of deformations

$$
\Phi^{(i)}:(0,1] \times \mathcal{V}_{\kappa}\left(\gamma_{i}\right) \rightarrow \mathcal{V}_{\kappa}\left(\gamma_{i}\right), \quad i=1, \ldots, g,
$$

pinching along $\gamma_{i}$. Let $q \in \mathcal{V}_{\kappa}\left(\gamma_{i}\right)$ and let $\gamma_{i}(q)$ be the closed regular vertical $q$-trajectory isotopic to $\gamma_{i}$. Let then $\widehat{M}_{i}:=M \backslash\left\{\gamma_{i}(q)\right\}$. Let $\gamma_{i}^{(1)}, \gamma_{i}^{(2)}$ be the boundary components of $\widehat{M}_{i}$ produced by cutting along the regular trajectory $\gamma_{i}(q)$, endowed with the orientation induced by $\widehat{M}_{i}$, and let $\phi_{i}: \gamma_{i}^{(1)} \rightarrow \gamma_{i}^{(2)}$ be the corresponding (orientation reversing) attaching map. Let $D_{i}^{(k)}$ be copies of the closed unit disk in the complex plane and $h_{i}^{(k)}$ be the meromorphic 
differentials on $D_{i}^{(k)}, k=1,2$, given by

$$
h_{i}^{(k)}:=\frac{c_{i}^{(k)}}{2 \pi} \frac{d z_{i}^{(k)}}{z_{i}^{(k)}},
$$

where $c_{i}^{(k)} \neq 0$ are the periods of $\Im\left(q^{1 / 2}\right)$ along $\gamma_{i}^{(k)}$. Since $\gamma_{i}^{(k)}, k=1,2$, can be identified to $\gamma_{i}(q)$ taken with opposite orientations, we have that $c_{i}^{(1)}+c_{i}^{(2)}=0$. The boundary circle of $D_{i}^{(k)}$ is a regular vertical trajectory of $h_{i}^{(k)}$. Hence by gluing the pairs $\left(D_{i}^{(k)} \backslash\{0\}, h_{i}^{(k)}\right)$ to the pair $\left(\widehat{M}_{i}, q^{1 / 2}\right)$ along the curves $\gamma_{i}^{(k)}$, we obtain a pair $\left(M_{i}, q_{i}^{1 / 2}\right)$, where $M_{i}$ is a surface of genus $g-1$ with two (paired) punctures and $q_{i}^{1 / 2}$ is a regular 1 -differential with the same zeroes as $q^{1 / 2}$. Let $\psi_{i}^{(k)}: \partial D_{i}^{(k)} \rightarrow \gamma_{i}^{(k)}$ be the corresponding attaching map. Let $t_{i} \in(0,1]$, let $S_{i}^{(k)}\left(t_{i}\right)$ be the circle of radius $t_{i}$ in the disk $D_{i}^{(k)}$ and let $\psi_{i}\left(t_{i}\right): S_{i}^{(1)}\left(t_{i}\right) \rightarrow$ $S_{i}^{(2)}\left(t_{i}\right)$ be the attaching map defined as follows:

$$
\psi_{i}\left(t_{i}\right)\left(z_{i}^{(1)}\right):=t_{i}\left(\psi_{i}^{(2)}\right)^{-1} \circ \phi_{i} \circ \psi_{i}^{(1)}\left(t_{i}^{-1} z_{i}^{(1)}\right) .
$$

Let $\left(M_{i}\left(t_{i}\right), q_{i}\left(t_{i}\right)^{1 / 2}\right)$ be the pair obtained by restriction of $\left(M_{i}, q_{i}^{1 / 2}\right)$ to the complement of the open disk $\left\{\left|z_{i}^{(k)}\right|<t_{i}\right\} \subset D_{i}^{(k)}$ in $M_{i}$, then by gluing along the boundary curves in the way prescribed by the attaching map $\left(4.30^{\prime}\right)$. If $t_{i} \neq 0, M_{i}\left(t_{i}\right)$ is a compact Riemann surface of genus $g \geq 2$ and, since the boundary circles $S_{i}^{(k)}\left(t_{i}\right)$ are regular vertical trajectories of the quadratic differential $q_{i}, q_{i}\left(t_{i}\right)$ has the same zeroes as $q$, hence $q_{i}\left(t_{i}\right) \in Q_{\kappa}$. In addition, by the choice of the holomorphic differential (4.30) and of the attaching map $\left(4.30^{\prime}\right)$, the cohomology class $\left[\Im\left(q_{i}^{1 / 2}\left(t_{i}\right)\right)\right] \equiv\left[\Im\left(q^{1 / 2}\right)\right] \in H^{1}\left(M, \Sigma_{q} ; \mathbb{R}\right)$, for all $t_{i} \in(0,1]$. By construction, $\left(M_{i}\left(t_{i}\right), q_{i}\left(t_{i}\right)\right)=(M, q)$, if $t_{i}=1$, and $\left(M_{i}\left(t_{i}\right), q_{i}\left(t_{i}\right)\right)=\left(M_{i}, q_{i}\right)$, if $t_{i}=0$, where $q_{i}$ is a regular quadratic differential with two poles of order 2 at the two (paired) punctures of the surface $M_{i}$, with real strictly positive (equal) residues. We then define:

$$
\begin{array}{ll}
\text { (a) } \Phi_{t_{i}}^{(i)}(q):=q_{i}\left(t_{i}\right), & \left(t_{i}, q\right) \in(0,1] \times \mathcal{V}_{\kappa}\left(\gamma_{i}\right) ; \\
\text { (b) } \Phi_{t}(q):=\Phi_{t_{1}}^{(1)} \circ \cdots \circ \Phi_{t_{g}}^{(g)}(q), & (t, q) \in(0,1]^{g} \times \mathcal{V}_{\kappa}\left(\gamma_{1}, \ldots, \gamma_{g}\right)
\end{array}
$$

It can be checked that the pinching deformation $\Phi:(0,1]^{g} \times \mathcal{V}_{\kappa}\left(\gamma_{1}, \ldots, \gamma_{g}\right) \rightarrow$ $\mathcal{V}_{\kappa}\left(\gamma_{1}, \ldots, \gamma_{g}\right)$, given by (4.31), is well-defined and has the required properties. In fact, $\Phi_{t_{i}}^{(i)}\left(\mathcal{V}_{\kappa}\left(\gamma_{1}, \ldots, \gamma_{g}\right)\right) \subset \mathcal{V}_{\kappa}\left(\gamma_{1}, \ldots, \gamma_{g}\right)$, for all $i=1, \ldots, g$ and all $t_{i} \in(0,1]$. Since, by definition, $\Phi_{t_{i}}^{(i)}(q)=q$, if $t_{i}=1$, the property (1) holds. Let $\left(M_{0}, q_{0}\right)$ be the regular quadratic differential constructed by gluing all the pairs $\left(D_{i}^{(k)} \backslash\{0\}, h_{i}^{(k)}\right)$ to the pair $\left(\widehat{M}, q^{1 / 2}\right)$ along the curves $\gamma_{i}^{(k)}$. Then, since $\widehat{M}$ is homeomorphic to a sphere minus $2 g$ disjoint disks, $M_{0}$ is a punctured Riemann sphere (by the uniformization theorem) with $2 g$ paired punctures 
and $q_{0}$ is a regular quadratic differential with the same zeroes as $q$ and poles of order 2 at all punctures. Hence $q_{0} \in \mathcal{S}_{g}$ and in particular, by (4.30), it has strictly positive residues. Since $q_{t}:=\Phi_{t}(q) \rightarrow q_{0}$ as $t_{i} \rightarrow 0$, for all $i=1, \ldots, g$, property (2) is also proved. Finally, property (3), which will be relevant in a similar construction carried out in Section 8.2, follows, as we have remarked above, from the choice of the differentials (4.30) and of the attaching maps $\left(4.30^{\prime}\right)$.

THEOREM 4.5. No connected component $\mathcal{C}_{\kappa}$ of a stratum $\mathcal{M}_{\kappa}$ of holomorphic quadratic differentials is contained in the determinant locus. In fact, the following stronger result holds. Let $\mathcal{C}_{\kappa}^{(1)}:=\mathcal{C}_{\kappa} \cap \mathcal{M}_{\kappa}^{(1)}$. We have:

$$
\sup _{q \in \mathcal{C}_{\kappa}^{(1)}} \Lambda_{i}(q)=1, \text { for all } i \in\{1, \ldots, g\} \text {. }
$$

Proof. By the definition (4.2) it is sufficient to prove the statement for $i=g$. By Lemma $4.4^{\prime}, \mathcal{C}_{\kappa}$ has an accumulation point $\left(M_{0}, q_{0}\right) \in \mathcal{S}_{g}$ with real strictly positive residues at all $2 g$ (paired) punctures of the punctured Riemann sphere $M_{0}$. Let $\left(M_{(t, \tau)}, q_{(t, \tau)}\right),(t, \tau) \in \mathbb{C}^{g} \times \mathbb{C}^{2 g-3}$ be the holomorphic family, whose construction has been outlined above, parametrizing a neighbourhood of $\left(M_{0}, q_{0}\right)$ in the compactified moduli space of quadratic differentials. Then $\left(M_{(t, \tau)}, q_{(t, \tau)}\right)=\left(M_{0}, q_{0}\right)$, if $(t, \tau)=(0,0)$, and $\left(M_{(t, \tau)}, q_{(t, \tau)}\right) \in \mathcal{S}_{g}$, if $t=0$ and $\tau \in \mathbb{C}^{2 g-3}$ is close to the origin. Let $\Pi_{(t, \tau)}$ be the period matrix of $M_{(t, \tau)}$ with respect to a normalized basis $\left\{\theta_{1}(t, \tau), \ldots, \theta_{g}(t, \tau)\right\}$ on $M_{(t, \tau)}$, holomorphic in a neigbourhood of the origin in $\mathbb{C}^{g} \times \mathbb{C}^{2 g-3}$. The period matrix is well-defined if $t_{i} \neq 0$ for all $i=1, \ldots, g$. By [15, III] or [71, $\S 3$, Corollary 6] (see for instance $[15$, p. 54]), the period matrix satisfies the following asymptotics (taking into account that the normalization condition adopted by [15] and [71] differs from ours by a factor $2 \pi \imath)$ :

$$
\Pi_{(t, \tau)}^{i j}-\frac{1}{2 \pi \imath} \delta_{i j} \log t_{i}
$$

is bounded as $t \rightarrow 0$, uniformly on $\tau \in \mathbb{C}^{2 g-3}$ in a compact neighbourhood of the origin. Let $\Lambda_{i}(t, \tau), i \in\{1, \ldots, g\}$, be the eigenvalues (4.2) of the hermitian form $\left(4.2^{\prime}\right)$ at $\left(M_{(t, \tau)}, q_{(t, \tau)}\right)$. By formula (4.6), Lemmas 4.2 and $4.2^{\prime}$ and by the boundedness of $(4.33)$, we obtain:

$$
\lim _{(t, \tau) \rightarrow(0,0)} \Lambda_{1}(t, \tau) \cdots \Lambda_{g}(t, \tau)=1 .
$$

Since any neighbourhood of $\left(M_{0}, q_{0}\right)$ in the compactified moduli space contains points of the connected component $\mathcal{C}_{\kappa}$ and since the hermitian form $\left(4.2^{\prime}\right)$ and all its eigenvalues $\Lambda_{i}$ are invariant under the multiplicative $\mathbb{R}$-action on quadratic differentials, the statement is proved. 
Corollary 4.5'. The second Lyapunov exponents of the KontsevichZorich cocycle with respect to the ergodic invariant measure given by the restriction to any connected component $\mathcal{C}_{\kappa}^{(1)}$ of a stratum $\mathcal{M}_{\kappa}^{(1)}$ is strictly positive. In fact, it satisfies the estimate

$$
\lambda_{2}\left(\mathcal{C}_{\kappa}^{(1)}\right) \geq \frac{1}{\mu_{\kappa}^{(1)}\left(\mathcal{C}_{\kappa}^{(1)}\right)} \int_{\mathcal{C}_{\kappa}^{(1)}} \Lambda_{g} d \mu_{\kappa}^{(1)}>0 .
$$

Proof. The first inequality in (4.35) follows from the ergodicity of the Teichmüller flow on $\mathcal{C}_{\kappa}^{(1)}$ (see Theorem 1.1) and from the lower bound proved in Theorem 3.3. In fact, $\Lambda^{-} \equiv \Lambda_{g}$, by the definitions (3.15), (4.2), (4.2') and standard properties of hermitian forms. Finally, the strict positivity of the integral in (4.35) holds by Theorem 4.5, since the function $\Lambda_{g}$ is continuous and the measure $\mu_{\kappa}^{(1)}$ is positive on any open set.

\section{The Kontsevich-Zorich formula revisited and other formulas for the Lyapunov exponents}

In [38] M. Kontsevich and A. Zorich obtained a formula for the sum $\lambda_{1}+\cdots+\lambda_{g}$ of the non-negative Lyapunov exponents of the Kontsevich-Zorich cocycle. In this section we prove a different version of the formula along the lines of Sections 2 and 3. The same method yields formulas for all the partial sums $\lambda_{1}+\cdots+\lambda_{k}, 1 \leq k \leq g$.

Let $G_{k}(M, \mathbb{R}) \subset H^{1}(M, \mathbb{R})$ be the Grassmannian of isotropic subspaces of dimension $k \in\{1, \ldots, g\}$, which is a compact manifold of dimension $(2 g-k) k-\left(k^{2}-k\right) / 2$. Let $q \in Q_{\kappa}^{(1)}$ be an orientable quadratic differential and $I_{k} \in G_{k}(M, \mathbb{R})$ an isotropic $k$-plane. Let $\mathcal{S}_{k}:=\left\{c_{1}, \ldots, c_{k}\right\}$ be an ordered basis of $I_{k}$. By (2.4) such a basis corresponds to an ordered system $\left\{m_{1}^{+}, \ldots, m_{k}^{+}\right\}$, linearly independent over $\mathbb{R}$, of meromorphic functions in $L_{q}^{2}(M)$. Let $\left\{v_{1}, \ldots, v_{k}\right\} \subset H^{1}(M)$ be a system of the functions determined (up to additive constants) by the orthogonal decompositions

$$
m_{i}^{+}=\partial_{q}^{+} v_{i}+\pi_{q}^{-}\left(m_{i}^{+}\right),
$$

where $\pi_{q}^{-}: L_{q}^{2}(M) \rightarrow \mathcal{M}_{q}^{-}$is the orthogonal projection operator given by the splitting (2.3). Let $A^{k}, H^{k}, B^{k}$ and $V^{k}$ be the $k \times k$ matrices defined as follows:

$$
\begin{aligned}
A_{i j}^{k} & :=\left(m_{i}, m_{j}\right)_{q}, \\
H_{i j}^{k} & :=\left(\pi_{q}^{-}\left(m_{i}^{+}\right), \pi_{q}^{-}\left(m_{j}^{+}\right)\right)_{q}, \\
B_{i j}^{k} & :=B_{q}\left(m_{i}^{+}, m_{j}^{+}\right):=\left(m_{i}^{+}, \overline{m_{j}^{+}}\right)_{q}, \\
V_{i j}^{k} & :=\left(\partial_{q}^{+} v_{i}, \partial_{q}^{+} \overline{v_{j}}\right)_{q}=\left(\partial_{q}^{-} v_{i}, \partial_{q}^{-} \overline{v_{j}}\right)_{q} .
\end{aligned}
$$


Such matrices depend on the pair $\left(q, \mathcal{S}_{k}\right)$, where $q \in Q_{\kappa}^{(1)}$ and $\mathcal{S}_{k}$ is an ordered linearly independent isotropic system of length $k \in\{1, \ldots, g\}$ of real cohomology classes. However they are invariant under the natural action of the pure mapping class group $\Gamma_{g}$. By the isotropy property and $\left(2.5^{\prime}\right)$, the matrix $A^{k}$ is real and symmetric, while $H^{k}$ is hermitian and $B^{k}, V^{k}$ are complex and symmetric.

Our goal is to compute the evolution of the $k$-dimensional volume on any isotropic plane $I_{k}$ under the action of the Kontsevich-Zorich cocycle. Let $q \in Q_{\kappa}^{(1)}$ and let $\mathcal{S}_{k}:=\left\{c_{1}, \ldots, c_{k}\right\}$ be any linearly independent ordered isotropic system of cohomology classes.

LemmA 5.1. Let $q_{t}:=G_{t}(q), \mathcal{S}_{k}(t):=G_{t}^{K Z}\left(\mathcal{S}_{k}\right)$ and $A_{t}^{k}, H_{t}^{k}, B_{t}^{k}$ and $V_{t}^{k}$ be the matrices associated to the pair $\left(q_{t}, \mathcal{S}_{k}(t)\right)$ according to (5.2). The following formulas hold:

$$
\begin{aligned}
\frac{d}{d t} \operatorname{det}\left(A_{t}^{k}\right)= & -2 \operatorname{det}\left(A_{t}^{k}\right) \operatorname{tr}\left[\left(A_{t}^{k}\right)^{-1} \Re B_{t}^{k}\right], \\
\frac{d^{2}}{d t^{2}} \operatorname{det}\left(A_{t}^{k}\right)= & 4 \operatorname{det}\left(A_{t}^{k}\right)\left\{\operatorname{tr}^{2}\left[\left(A_{t}^{k}\right)^{-1} \Re B_{t}^{k}\right]\right. \\
& \left.-\operatorname{tr}\left[\left(A_{t}^{k}\right)^{-1} \Re B_{t}^{k}\right]^{2}+\operatorname{tr}\left[\left(A_{t}^{k}\right)^{-1} \Re\left(H_{t}^{k}-V_{t}^{k}\right)\right]\right\} .
\end{aligned}
$$

Proof. By the standard formulas for the derivatives of a determinant:

$$
\begin{aligned}
\frac{d}{d t} \operatorname{det}\left(A_{t}^{k}\right)= & \operatorname{det}\left(A_{t}^{k}\right) \operatorname{tr}\left[\left(A_{t}^{k}\right)^{-1} \frac{d}{d t} A_{t}^{k}\right] \\
\frac{d^{2}}{d t^{2}} \operatorname{det}\left(A_{t}^{k}\right)= & \operatorname{det}\left(A_{t}^{k}\right)\left\{\operatorname{tr}^{2}\left[\left(A_{t}^{k}\right)^{-1} \frac{d}{d t} A_{t}^{k}\right]\right. \\
& \left.+\operatorname{tr}\left[\left(A_{t}^{k}\right)^{-1} \frac{d^{2}}{d t^{2}} A_{t}^{k}-\left(\left(A_{t}^{k}\right)^{-1} \frac{d}{d t} A_{t}^{k}\right)^{2}\right]\right\} .
\end{aligned}
$$

In addition, by the variational formulas (2.12) for the Kontsevich-Zorich cocycle, proved in Lemma 2.1, we can compute

$$
\begin{aligned}
\frac{d}{d t} A_{t}^{k} & =-2 \Re B_{t}^{k}, \\
\frac{d^{2}}{d t^{2}} A_{t}^{k} & =4 \Re\left(H_{t}^{k}-V_{t}^{k}\right) .
\end{aligned}
$$

The formulas in (5.3) are then obtained by (5.4) and $\left(5.4^{\prime}\right)$.

We can then compute the following generalization of the formula of Lemma 3.2. Let $q \in Q_{\kappa}^{(1)}$ and $q_{z}, z \in D$, be given by (3.1). Let $I_{k} \in G_{k}(M, \mathbb{R})$ be an isotropic $k$-plane and $\mathcal{S}_{k}$ be any given ordered basis of $I_{k}$. Let $A_{z}^{k}, H_{z}^{k}, B_{z}^{k}$ and $V_{z}^{k}$ be the matrices associated to the pair $\left(q_{z}, \mathcal{S}_{k}\right)$ according to (5.2).

LEMma 5.2. The following formulas hold:

$$
\triangle_{h} \log \left|\operatorname{det}\left(A_{z}^{k}\right)\right|^{1 / 2}=4 \operatorname{tr}\left[\left(A_{z}^{k}\right)^{-1} H_{z}^{k}\right]-2 \operatorname{tr}\left[\left(A_{z}^{k}\right)^{-1} B_{z}^{k}\left(A_{z}^{k}\right)^{-1} \overline{B_{z}^{k}}\right] .
$$


Proof. The argument follows the proof of Lemma 3.2. It suffices to prove (5.5) at the origin of the Poincaré disk $D$. Let $X_{\theta}$ be the directional derivative at $z=0$, defined by (3.9). By Lemma 5.1 and the identities (3.11) and (3.11'), we have:

$$
\begin{aligned}
X_{\theta} \operatorname{det}\left(A_{z}^{k}\right)= & -2 \operatorname{det}\left(A_{0}^{k}\right) \operatorname{tr}\left[\left(A_{0}^{k}\right)^{-1} \Re\left(e^{-i \theta} B_{0}^{k}\right)\right], \\
X_{\theta}^{2} \operatorname{det}\left(A_{z}^{k}\right)= & 4 \operatorname{det}\left(A_{0}^{k}\right)\left\{\operatorname{tr}^{2}\left[\left(A_{0}^{k}\right)^{-1} \Re\left(e^{-i \theta} B_{0}^{k}\right)\right]\right. \\
& \left.-\operatorname{tr}\left[\left(A_{0}^{k}\right)^{-1} \Re\left(e^{-i \theta} B_{0}^{k}\right)\right]^{2}+\operatorname{tr}\left[\left(A_{0}^{k}\right)^{-1} \Re\left(H_{0}^{k}-e^{-2 i \theta} V_{0}^{k}\right)\right]\right\} .
\end{aligned}
$$

We can write the hyperbolic gradient and Laplacian in terms of the directional derivatives as in (3.12):

$$
\begin{aligned}
\nabla_{h} \operatorname{det}\left(A_{z}^{k}\right)= & \left(X_{0} \operatorname{det}\left(A_{z}^{k}\right), X_{\frac{\pi}{2}} \operatorname{det}\left(A_{z}^{k}\right)\right) \\
= & -2 \operatorname{det}\left(A_{0}^{k}\right)\left(\operatorname{tr}\left[\left(A_{0}^{k}\right)^{-1} \Re B_{0}^{k}\right], \operatorname{tr}\left[\left(A_{0}^{k}\right)^{-1} \Im B_{0}^{k}\right]\right), \\
\triangle_{h} \operatorname{det}\left(A_{z}^{k}\right)= & \frac{1}{\pi} \int_{0}^{2 \pi} X_{\theta}^{2} \operatorname{det}\left(A_{z}^{k}\right) d \theta \\
= & 4 \operatorname{det}\left(A_{0}^{k}\right)\left\{\operatorname{tr}^{2}\left[\left(A_{0}^{k}\right)^{-1} \Re B_{0}^{k}\right]+\operatorname{tr}^{2}\left[\left(A_{0}^{k}\right)^{-1} \Im B_{0}^{k}\right]\right. \\
& \left.-\operatorname{tr}\left[\left(A_{0}^{k}\right)^{-1} \Re B_{0}^{k}\right]^{2}-\operatorname{tr}\left[\left(A_{0}^{k}\right)^{-1} \Im B_{0}^{k}\right]^{2}+2 \operatorname{tr}\left[\left(A_{0}^{k}\right)^{-1} \Re H_{0}^{k}\right]\right\} .
\end{aligned}
$$

The desired formula (5.5) (at $z=0$ ) can be derived from $\left(5.6^{\prime}\right)$ by applying the formula (3.13) with $\phi(z):=\left|\operatorname{det}\left(A_{z}^{k}\right)\right|^{1 / 2}$.

Lemma $5.2^{\prime}$. The formula

$$
\Phi_{k}:=2 \operatorname{tr}\left[\left(A^{k}\right)^{-1} H^{k}\right]-\operatorname{tr}\left[\left(A^{k}\right)^{-1} B^{k}\left(A^{k}\right)^{-1} \overline{B^{k}}\right],
$$

where the matrices $A^{k}, H^{k}$ and $B^{k}$ are given by (5.2), is independent of the choice of a basis of the isotropic plane $I_{k}$ and therefore defines a nonnegative function $\Phi_{k}: Q_{\kappa}^{(1)} \times G_{k}(M, \mathbb{R}) \rightarrow \mathbb{R}$, invariant under the natural action of the modular group $\Gamma_{g}$. In addition, the following identities hold. Let $I_{1} \subset I_{2} \subset \cdots \subset I_{g} \subset H^{1}(M, \mathbb{R})$ be a finite sequence of isotropic subspaces such that $\operatorname{dim}\left(I_{k}\right)=k \in\{1, \ldots, g\}$. Let $q \in Q_{\kappa}^{(1)}$ and $\left\{m_{1}^{+}, \ldots, m_{g}^{+}\right\}$be an orthonormal basis of the space $\mathcal{M}_{q}^{+}$of meromorphic functions in $L_{q}^{2}(M)$ such that, for each $k \in\{1, \ldots, g\},\left\{m_{1}^{+}, \ldots, m_{k}^{+}\right\}$represents an orthonormal basis of $I_{k}$, in the sense that, if $c_{i}=\left[\Re\left(m_{i}^{+} q^{1 / 2}\right)\right] \in H^{1}(M, \mathbb{R})$, then $\left\{c_{1}, \ldots, c_{k}\right\}$ is an orthonormal basis of $I_{k}$ with respect to the Hodge norm induced by the metric $R_{q}$. Then

(1) $\Phi_{1}\left(q, I_{1}\right)=2\left|\pi_{q}^{-}\left(m_{1}^{+}\right)\right|^{2}-\left|B_{q}\left(m_{1}^{+}\right)\right|^{2}$,

(2) $\Phi_{k}\left(q, I_{k}\right)=\Phi_{g}\left(q, I_{g}\right)-\sum_{i, j=k+1}^{g}\left|B_{q}\left(m_{i}^{+}, m_{j}^{+}\right)\right|^{2}$,

(3) $\Phi_{g}\left(q, I_{g}\right) \equiv \Lambda_{1}(q)+\cdots+\Lambda_{g}(q)$. 
Proof. The formula (5.7) depends in principle on the choice of a basis $\left\{c_{1}, \ldots, c_{k}\right\}$ of the isotropic subspace $I_{k}$. However, since the matrix of a change of base is a real matrix, a computation using the commutativity property of the trace shows that in fact there is no dependence on the choice of the base. In particular, if we choose an orthonormal basis, then by the isotropy of $I_{k}$, $\left(m_{i}^{+}, m_{j}^{+}\right)_{q}=\delta_{i j}$ and we obtain the following formula:

$$
\Phi_{k}\left(q, I_{k}\right)=2 \sum_{i=1}^{k}\left|\pi_{q}^{-}\left(m_{i}^{+}\right)\right|^{2}-\sum_{i, j=1}^{k}\left|B_{q}\left(m_{i}^{+}, m_{j}^{+}\right)\right|^{2} .
$$

The identity (1) is a particular case of $\left(5.7^{\prime}\right)$. The identity (2) can be computed on the basis of $\left(5.7^{\prime}\right)$ taking into account the following identity, which holds since $\left\{m_{1}^{+}, \ldots, m_{g}^{+}\right\}$, and hence $\left\{\overline{m_{1}^{+}}, \ldots, \overline{m_{g}^{+}}\right\}$, are orthonormal bases for the spaces of meromorphic, respectively anti-meromorphic, functions in $L_{q}^{2}(M)$ :

$$
\pi_{q}^{-}\left(m_{i}^{+}\right)=\sum_{j=1}^{g} B_{q}\left(m_{i}^{+}, m_{j}^{+}\right) \overline{m_{j}^{+}} .
$$

Finally, if $k=g$, by $\left(5.7^{\prime}\right)$ and (5.8),

$$
\Phi_{g}\left(q, I_{g}\right)=\sum_{i=1}^{g}\left|\pi_{q}^{-}\left(m_{i}^{+}\right)\right|^{2}=\Lambda_{1}(q)+\cdots+\Lambda_{g}(q),
$$

which proves (3). The invariance of $\Phi_{k}$ under the natural action of the modular group follows from the invariance of the matrices $A^{k}, H^{k}, B^{k}$, which enter in the definition (5.7).

Let $q \in Q_{\kappa}^{(1)}, z \equiv(t, \theta) \in D$ in geodesics polar coordinates on the Teichmüller disk centered at $q$ and let $q_{z}$ be defined as in (3.1). Let $G_{z}^{K Z}$ be given by the (trivial) parallel transport of cohomology classes from the origin at $q$ to $q_{z}$ along the Teichmüller disk centered at $q$ in $Q_{\kappa}^{(1)}$. By Lemma 3.1 and Lemma 5.2,

$$
\frac{1}{2 \pi} \frac{\partial}{\partial t} \int_{0}^{2 \pi} \log \left|\operatorname{det} A_{z}^{k}\right|^{1 / 2} d \theta=\tanh (t) \frac{1}{\left|D_{t}\right|} \int_{D_{t}} \Phi_{k} \circ G_{\xi}^{K Z}\left(q, I_{k}\right) \omega_{P}(\xi)
$$

As a first consequence of (5.10), we prove the following version of the formula for the sum of the first $g$ exponents, given by M. Kontsevich and A. Zorich [38]:

COROLlary 5.3. The Lyapunov exponents of the Kontsevich-Zorich cocycle with respect to the ergodic invariant measure given by the restriction to any connected component $\mathcal{C}_{\kappa}^{(1)}$ of a stratum $\mathcal{M}_{\kappa}^{(1)}$ satisfy the following identity:

$$
\lambda_{1}+\cdots+\lambda_{g}=\frac{1}{\mu_{\kappa}^{(1)}\left(\mathcal{C}_{\kappa}^{(1)}\right)} \int_{\mathcal{C}_{\kappa}^{(1)}}\left(\Lambda_{1}+\cdots+\Lambda_{g}\right) d \mu_{\kappa}^{(1)} .
$$


Proof. Let $\sigma_{q}^{k}$ be the normalized canonical (Haar) measure on the Grassmannian $G_{k}(M, \mathbb{R})$ of isotropic $k$-dimensional subspaces $I_{k} \subset H^{1}\left(M_{q}, \mathbb{R}\right)$, endowed with the Euclidean structure given by the Hodge inner product induced by the metric $R_{q}$. The probability measure $\sigma_{q}^{k}$ is invariant under the action of the circle group $\mathrm{SO}(2, \mathbb{R})$ on $Q_{g}$. In the case $k=g$, since the function $\Phi_{g}$ does not depend on the Lagrangian plane $I_{g}$, by averaging $(5.10)$ over $G_{k}(M, \mathbb{R})$ with respect to $\sigma_{q}^{g}$, then over a stratum $\mathcal{M}_{\kappa}^{(1)}$ with respect to any ergodic $G_{t}$-invariant measure $\mu$, we obtain:

$$
\begin{aligned}
\frac{1}{2 \pi} \frac{\partial}{\partial t} \int_{\mathcal{M}_{\kappa}^{(1)}} \int_{G_{k}(M, \mathbb{R})} \int_{0}^{2 \pi} & \log \left|\operatorname{det} A_{z}^{g}\right|^{1 / 2} d \theta d \sigma_{q}^{g} d \mu \\
& =\tanh (t) \int_{\mathcal{M}_{\kappa}^{(1)}}\left(\Lambda_{1}+\cdots+\Lambda_{g}\right) d \mu .
\end{aligned}
$$

If $\mu$ is the normalized restriction to any connected component of $\mathcal{M}_{\kappa}^{(1)}$ of the invariant measure $\mu_{\kappa}^{(1)}$, which is absolutely continuous and invariant under the action of the circle group on $Q_{\kappa}^{(1)}$, by applying Fubini's theorem to the left-hand side of (5.12) and averaging over $[0, \mathcal{T}]$ with respect to time, we have:

$$
\begin{aligned}
\frac{1}{\mathcal{T}} \int_{\mathcal{M}_{\kappa}^{(1)}} \int_{G_{k}(M, \mathbb{R})} \log \left|\operatorname{det}\left(A_{\mathcal{T}}^{g}\left(A_{0}^{g}\right)^{-1}\right)\right|^{1 / 2} d \sigma_{q}^{k} d \mu \\
=\frac{\log \cosh \mathcal{T}}{\mathcal{T}} \int_{\mathcal{M}_{\kappa}^{(1)}}\left(\Lambda_{1}+\cdots+\Lambda_{g}\right) d \mu .
\end{aligned}
$$

The identity $\left(5.12^{\prime}\right)$ yields $(5.11)$ in the limit $\mathcal{T} \rightarrow+\infty$. In fact, by Oseledec's theorem, the limit of the left-hand side is equal to the sum $\lambda_{1}+\cdots+\lambda_{g}$.

Remark 5.3'. Since $\Lambda_{1} \equiv \lambda_{1}=1$, the formula (5.11) is equivalent to the following:

$$
\lambda_{2}+\cdots+\lambda_{g}=\frac{1}{\mu_{\kappa}^{(1)}\left(\mathcal{C}_{\kappa}^{(1)}\right)} \int_{\mathcal{C}_{\kappa}^{(1)}}\left(\Lambda_{2}+\cdots+\Lambda_{g}\right) d \mu_{\kappa}^{(1)} .
$$

In particular, such formula implies that $\lambda_{2}>0$ (Corollary $4.5^{\prime}$ ) and it gives an exact formula for the second Lyapunov exponent in the case $g=2$.

The formulas (5.10) directly imply the non-vanishing of about a half of Lyapunov exponents in case $g>2$.

COROLlary 5.4. Let us consider the Lyapunov spectrum of the Kontsevich-Zorich cocycle with respect to the restriction of the measure $\mu_{\kappa}^{(1)}$ to any connected component of $\mathcal{M}_{\kappa}^{(1)}$. If $k<g / 2$,

$$
1 \equiv \lambda_{1}>\lambda_{2} \geq \cdots \geq \lambda_{k} \geq \lambda_{k+1}>0
$$


Proof. Let $k<g / 2$ and let

$$
M_{k}(q):=\sup \left\{\Phi_{k}\left(q, I_{k}\right) \mid I_{k} \in G_{k}(M, \mathbb{R})\right\} .
$$

We claim that any connected component of the stratum $\mathcal{M}_{\kappa}^{(1)}$ has an open subset where the following inequality holds:

$$
M_{k}(q)<\Lambda_{1}(q)+\cdots+\Lambda_{g}(q) .
$$

In fact, if $\left(5.15^{\prime}\right)$ fails, by Lemma $5.2^{\prime}$ and the compactness of the Grassmannian, there exists an orthonormal system $\left\{m_{1}^{+}, \ldots, m_{g}^{+}\right\} \subset \mathcal{M}_{q}^{+}$such that $B_{q}\left(m_{i}^{+}, m_{j}^{+}\right)=0$ for all $i, j \in\{k+1, \ldots, g\}$. By (5.8), if the projection $\pi_{q}^{-}$is injective, then $g-k \leq k$. Since $k<g / 2$ and, by Theorem $4.5, \pi_{q}^{-}$is injective on an open subset of any connected component of $\mathcal{M}_{\kappa}^{(1)}$, the claim is proved. By formula (5.10),

$$
\frac{1}{2 \pi} \frac{\partial}{\partial t} \int_{0}^{2 \pi} \log \left|\operatorname{det} A_{z}^{k}\right|^{1 / 2} d \theta \leq \tanh (t) \frac{1}{\left|D_{t}\right|} \int_{D_{t}} M_{k}\left(q_{\xi}\right) \omega_{P}(\xi) .
$$

Then, by averaging successively over $G_{k}(M, \mathbb{R})$ with respect to $\sigma_{q}^{k}$, over a connected component $\mathcal{C}_{\kappa}^{(1)} \subset \mathcal{M}_{\kappa}^{(1)}$ with respect to the normalized restriction $\mu$ of the measure $\mu_{\kappa}^{(1)}$ and over the interval $[0, \mathcal{T}]$ with respect to time, we obtain:

$$
\begin{aligned}
\frac{1}{\mathcal{T}} \int_{\mathcal{M}_{\kappa}^{(1)}} \int_{G_{k}(M, \mathbb{R})} \log \left|\operatorname{det}\left(A_{\mathcal{T}}^{g}\left(A_{0}^{g}\right)^{-1}\right)\right|^{1 / 2} d \sigma_{q}^{k} d \mu & \\
& \leq \frac{\log \cosh \mathcal{T}}{\mathcal{T}} \int_{\mathcal{M}_{\kappa}^{(1)}} M_{k}(q) d \mu
\end{aligned}
$$

Finally, by taking the limit as $\mathcal{T} \rightarrow+\infty$, we have:

$$
\begin{aligned}
\lambda_{1}+\cdots+\lambda_{k} & \leq \frac{1}{\mu_{\kappa}^{(1)}\left(\mathcal{C}_{\kappa}^{(1)}\right)} \int_{\mathcal{C}_{\kappa}^{(1)}} M_{k}(q) d \mu_{\kappa}^{(1)} \\
& <\frac{1}{\mu_{\kappa}^{(1)}\left(\mathcal{C}_{\kappa}^{(1)}\right)} \int_{\mathcal{C}_{\kappa}^{(1)}}\left(\Lambda_{1}+\cdots+\Lambda_{g}\right) d \mu_{\kappa}^{(1)}=\lambda_{1}+\cdots+\lambda_{g} .
\end{aligned}
$$

Hence, $\lambda_{k+1}>0$. In fact, a strictly positive lower bound can be derived from (5.17).

The investigation of higher Lyapunov exponents requires some kind of control on the invariant sub-bundles of the Kontsevich-Zorich cocycle. A result in that direction will be proved in the next section. We conclude this section by deriving one more consequence of formula (5.10), an exact formula for the exponents in terms of the invariant sub-bundles.

Corollary 5.5. Assume $\lambda_{k}>\lambda_{k+1} \geq 0, k \in\{1, \ldots, g-1\}$. Let $E_{k}^{ \pm} \subset \mathcal{H}_{\kappa}^{1}(M, \mathbb{R})$ be the $k$-dimensional invariant sub-bundles corresponding respectively to the sets of Lyapunov exponents $\pm\left\{\lambda_{1}, \ldots, \lambda_{k}\right\}$ in the Oseledec's 
splitting of the Kontsevich-Zorich cocycle. Then the following formula holds:

$$
\lambda_{1}+\cdots+\lambda_{k}=\frac{1}{\mu_{\kappa}^{(1)}\left(\mathcal{C}_{\kappa}^{(1)}\right)} \int_{\mathcal{C}_{\kappa}^{(1)}} \Phi_{k}\left(q, E_{k}^{+}(q)\right) d \mu_{\kappa}^{(1)} .
$$

Proof. By Oseledec's theorem, for almost all $q \in \mathcal{M}_{\kappa}^{(1)}$, almost all $\theta \in[0,2 \pi]$ and $\sigma_{q}^{k}$-almost all $k$-dimensional isotropic subspaces $I_{k} \in G_{k}(M, \mathbb{R})$,

$$
\operatorname{dist}\left(G_{(t, \theta)}^{K Z}\left(q, I_{k}\right), G_{t}^{K Z}\left(q_{\theta}, E_{k}^{+}\left(q_{\theta}\right)\right)\right) \rightarrow 0,
$$

as $t \rightarrow+\infty$. Consequently, since the function $\Phi_{k}$ is bounded and continuous, by Fubini's theorem and the dominated convergence theorem,

$$
\begin{aligned}
\frac{1}{\left|D_{t}\right|} \int_{G_{k}(M, \mathbb{R})} \int_{D_{t}} \mid \Phi_{k} \circ G_{(s, \theta)}^{K Z}\left(q, I_{k}\right) \\
-\Phi_{k} \circ G_{s}^{K Z}\left(q_{\theta}, E_{k}^{+}\left(q_{\theta}\right)\right) \mid \omega_{P}(s, \theta) d \sigma_{q}^{k} \rightarrow 0,
\end{aligned}
$$

as $t \rightarrow+\infty$, for almost all $q \in \mathcal{M}_{\kappa}^{(1)}$. Hence, by averaging $(5.10)$ over $G_{k}(M, \mathbb{R})$ with respect to the measure $\sigma_{q}^{k}$, then with respect to the normalized restriction $\mu$ of the invariant measure $\mu_{\kappa}^{(1)}$ to a connected component $\mathcal{C}_{\kappa}^{(1)} \subset \mathcal{M}_{\kappa}^{(1)}$ and applying Fubini's theorem, we find that, since $E_{k}^{+}$is an invariant sub-bundle of $G^{K Z}$ and $\mu$ is $\operatorname{SL}(2, \mathbb{R})$-invariant,

$$
\begin{aligned}
\frac{\partial}{\partial t} \int_{\mathcal{M}_{\kappa}^{(1)}} \int_{G_{k}(M, \mathbb{R})} & \log \left|\operatorname{det}\left(A_{t}^{k}\right)\right|^{1 / 2} d \sigma_{q}^{k} d \mu \\
& -\tanh (t) \int_{\mathcal{M}_{\kappa}^{(1)}} \Phi_{k}\left(q, E_{k}^{+}(q)\right) d \mu \rightarrow 0,
\end{aligned}
$$

as $t \rightarrow+\infty$. Finally, by averaging over $[0, \mathcal{T}]$ with respect to time and by Oseledec's theorem, we obtain (5.18).

\section{Basic currents for measured foliations}

The invariant unstable and stable subspaces of the Kontsevich-Zorich cocycle at a quadratic differential $q \in \mathcal{M}_{\kappa}^{(1)}$ will be described in terms of distributional invariants of, respectively, its horizontal and vertical foliations, $\mathcal{F}_{q}$ and $\mathcal{F}_{-q}$. Such invariants will be defined as a distributional generalization of the notion of basic form, well known in the geometric theory of foliations [53], [54], [63, Chap. 4], [2, §1.5 \& §7] and will therefore be called basic currents. In this section we prove a few properties of basic currents and of the weighted Sobolev spaces introduced in $[18, \S 2]$ which will be relevant in the study of the Kontsevich-Zorich cocycle. The most crucial is a version of the Poincaré 
inequality with an explicit geometric estimate for the constant. Such an estimate, equivalent to a lower bound for the first non-trivial eigenvalue of the Dirichlet form of the metric $R_{q}$, is obtained following Cheeger's method [9], [4].

6.1. Basic currents and invariant distributions. Let $\mathcal{F}$ be a measured foliation on a compact orientable surface $M$ of genus $g \geq 2$ in the sense of Thurston [62], [14]. Let $\Sigma \subset M$ be its (finite) set of singularities.

Definition 6.1. A basic current for $\left.\mathcal{F}\right|_{M \backslash \Sigma}$ is a current $C \in \mathcal{D}^{\prime}(M \backslash \Sigma$ ) (in the sense of de Rham [11], see also [56, Chap. IX]), homogeneous of dimension (and degree) equal to 1, with the following property:

$$
\imath_{X} C=\mathcal{L}_{X} C=0,
$$

for all smooth vector fields $X$ tangent to $\mathcal{F}$, with compact support in $M \backslash \Sigma$.

The operators of contraction and Lie derivative, with respect to a smooth vector field $X$, denoted $\imath_{X}$ and $\mathcal{L}_{X}$ respectively, are extended to currents in the standard distributional (weak) sense [56, Chap. IX, §3]. The vector space of all real basic currents for $\left.\mathcal{F}\right|_{M \backslash \Sigma}$ will be denoted by $\mathcal{B}_{\mathcal{F}}(M \backslash \Sigma)$.

Let $q \in Q_{\kappa}$ be an orientable (holomorphic) quadratic differential on a (marked) Riemann surface $M_{q}$. Let $\Sigma_{q}$ be the (finite) set of its zeroes. We introduce the following space $\Omega_{q}(M)$ of smooth test forms on $M$. Let $p \in \Sigma_{q}$ be a zero of (even) order $k \in \mathbb{N}$. There exists a canonical complex coordinate $z: \mathcal{U}_{p} \rightarrow \mathbb{C}$ on a neighbourhood $\mathcal{U}_{p}$ such that $p \in \mathcal{U}_{p}, z(p)=0$ and $q=z^{k} d z^{2}$ on $\mathcal{U}_{p}$. Let $\pi_{p}: \mathcal{U}_{p} \rightarrow \mathbb{C}$ be the (local) covering map defined by $\pi_{p}(z):=$ $z^{k / 2+1} /(k / 2+1)$. A form $\alpha \in \Omega_{q}(M)$ if and only if, for all $p \in \Sigma_{q}$, there exists a smooth form $\lambda_{p}$ on a neighbourhood of $0 \in \mathbb{C}$ such that $\alpha=\pi_{p}^{*}\left(\lambda_{p}\right)$ on $\mathcal{U}_{p}^{\prime} \subset \mathcal{U}_{p}$. The dual space $\mathcal{S}_{q}^{\prime}(M)$ of $\Omega_{q}(M)$ will be called the space of $q$-tempered currents on $M$. A homogeneous tempered current of dimension $d$ will be a continuous functional on the subset $\Omega_{q}^{d}(M) \subset \Omega_{q}(M)$ of homogeneous forms of degree $d \in \mathbb{N}$.

Let $\mathcal{V}_{q}(M)$ be the space of vector fields $X$ on $M \backslash \Sigma_{q}$ such that $\imath_{X} \alpha$, $\mathcal{L}_{X} \alpha \in \Omega_{q}(M)$ for all $\alpha \in \Omega_{q}(M)$.

Definition $6.1^{\prime}$. A current $C \in \mathcal{S}_{q}^{\prime}(M)$, of degree and dimension equal to 1 , is basic for $\mathcal{F}_{ \pm q}$ if the identities (6.1) holds in $\mathcal{S}_{q}^{\prime}(M)$ for all $X \in \mathcal{V}_{q}(M)$, tangent to $\mathcal{F}_{ \pm q}$ on $M \backslash \Sigma_{q}$. The vector spaces of real $\mathcal{F}_{ \pm q}$-basic currents will be denoted, respectively, by $\mathcal{B}_{ \pm q}(M)$.

Let $\mathcal{Z}^{\prime}\left(M \backslash \Sigma_{q}\right) \subset \mathcal{D}^{\prime}\left(M \backslash \Sigma_{q}\right)$ be the vector space of closed real homogeneous currents of dimension (and degree) equal to 1 . A current $C \in \mathcal{D}^{\prime}\left(M \backslash \Sigma_{q}\right)$ is closed if the identity $d C=0$ holds in $\mathcal{D}^{\prime}\left(M \backslash \Sigma_{q}\right)$ [11, Chap. IV, §18]. By 
the generalized de Rham theorem $[11, \S 15$, Th. 12], [56, Chap. IX, §3, Th. I], there exists a natural cohomology map

$$
j_{q}: \mathcal{Z}^{\prime}\left(M \backslash \Sigma_{q}\right) \rightarrow H^{1}\left(M \backslash \Sigma_{q}, \mathbb{R}\right) .
$$

In fact, any closed current $C \in \mathcal{Z}^{\prime}\left(M \backslash \Sigma_{q}\right)$ defines a linear functional on the de Rham cohomology with compact supports $H_{c}^{1}\left(M \backslash \Sigma_{q}, \mathbb{R}\right)$ and, by Poincaré duality, $H_{c}^{1}\left(M \backslash \Sigma_{q}, \mathbb{R}\right)^{*} \equiv H^{1}\left(M \backslash \Sigma_{q}, \mathbb{R}\right)$. Let $\mathcal{Z}_{q}^{\prime}(M) \subset \mathcal{Z}^{\prime}\left(M \backslash \Sigma_{q}\right)$ be the subspace of $q$-tempered currents $C$ of dimension (and degree) equal to 1 which are closed in $\mathcal{S}_{q}^{\prime}(M)$, in the sense that $d C=0$ holds in $\mathcal{S}_{q}^{\prime}(M)$.

Lemma 6.2. The cohomology map (6.2) has the property that

$$
j_{q}: \mathcal{Z}_{q}^{\prime}(M) \rightarrow H^{1}(M, \mathbb{R}) \subset H^{1}\left(M \backslash \Sigma_{q}, \mathbb{R}\right) .
$$

Proof. Let $C \in \mathcal{Z}_{q}^{\prime}(M)$. Then $C \in \mathcal{Z}^{\prime}\left(M \backslash \Sigma_{q}\right)$ and the cohomology class $j_{q}(C) \in H^{1}\left(M \backslash \Sigma_{q}, \mathbb{R}\right)$ is therefore well-defined. Let $c \in H_{c}^{1}\left(M \backslash \Sigma_{q}, \mathbb{R}\right)$ belong to the kernel of the (surjective) canonical map

$$
\epsilon: H_{c}^{1}\left(M \backslash \Sigma_{q}, \mathbb{R}\right) \rightarrow H^{1}(M, \mathbb{R}) .
$$

Then $c=[d v]$, where $v$ is a smooth function on $M$ constant in a neighbourhood of each point $p \in \Sigma_{q}$. Since $v \in \Omega_{q}^{0}(M)$ and $C$ is closed in $\mathcal{S}_{q}^{\prime}(M), C(d v)=$ $d C(v)=0$. Hence $j_{q}(C) \in N(\epsilon)^{\perp}$, the annihilator of the kernel $N(\epsilon)$. The dual map

$$
\epsilon^{*}: H^{1}(M, \mathbb{R})^{*} \rightarrow H^{1}\left(M \backslash \Sigma_{q}, \mathbb{R}\right)
$$

coincides with the canonical injection $H^{1}(M, \mathbb{R}) \rightarrow H^{1}\left(M \backslash \Sigma_{q}, \mathbb{R}\right)$, under the identification $H^{1}(M, \mathbb{R})^{*} \equiv H^{1}(M, \mathbb{R})$ given by the intersection form on $H^{1}(M, \mathbb{R})$. Since $N(\epsilon)^{\perp}$ is equal to the range of the dual map $\epsilon^{*}, j_{q}(C) \epsilon$ $H^{1}(M, \mathbb{R})$.

Let $\mathcal{B}_{ \pm q}\left(M \backslash \Sigma_{q}\right):=\mathcal{B}_{\mathcal{F}}\left(M \backslash \Sigma_{q}\right), \mathcal{F}=\mathcal{F}_{ \pm q}$.

LEMma 6.2'. The following inclusions hold:

(1) $\mathcal{B}_{ \pm q}(M) \subset \mathcal{B}_{ \pm q}\left(M \backslash \Sigma_{q}\right)$,

(2) $\mathcal{B}_{ \pm q}(M \backslash \Sigma) \subset \mathcal{Z}^{\prime}\left(M \backslash \Sigma_{q}\right)$,

(3) $\mathcal{B}_{ \pm q}(M) \subset \mathcal{Z}_{q}^{\prime}(M)$.

Proof. (1) follows directly from the definitions. In fact, since smooth forms with compact support in $M \backslash \Sigma_{q}$ belong to the space $\Omega_{q}(M)$, the space of $q$-tempered currents $\mathcal{S}_{q}^{\prime}(M) \subset \mathcal{D}^{\prime}\left(M \backslash \Sigma_{q}\right)$ and vector fields with compact support in $M \backslash \Sigma_{q}$ belong to the space $\mathcal{V}_{q}(M)$. 
(2) The identity

$$
\mathcal{L}_{X} C=\imath_{X} d C+d\left(\imath_{X} C\right)
$$

holds for currents [56, (IX,3;32)] in $\mathcal{D}^{\prime}\left(M \backslash \Sigma_{q}\right)$ if $C \in \mathcal{D}^{\prime}\left(M \backslash \Sigma_{q}\right)$ and $X$ is a vector field with compact support in $M \backslash \Sigma_{q}$. Hence, if $C \in \mathcal{B}_{ \pm q}\left(M \backslash \Sigma_{q}\right)$, $\imath_{X} d C=0$ in $\mathcal{D}^{\prime}\left(M \backslash \Sigma_{q}\right)$ for any vector field $X$, with compact support in $M \backslash \Sigma_{q}$, tangent to $\mathcal{F}_{ \pm q}$. Since $C$ has dimension (and degree) equal to $1, d C$ has dimension 0 (and degree 2). It follows that $d C=0$, hence $C$ is closed, in $\mathcal{D}^{\prime}\left(M \backslash \Sigma_{q}\right)$.

(3) The identity (6.4) holds in $\mathcal{S}_{q}^{\prime}(M)$ if $C \in \mathcal{S}_{q}^{\prime}(M)$ and $X \in \mathcal{V}_{q}(M)$. If $C \in \mathcal{B}_{ \pm q}(M)$, it follows by (6.4) that $\imath_{X} d C=0$ for all vector fields $X \in \mathcal{V}_{q}(M)$, tangent to $\mathcal{F}_{ \pm q}$. Hence $d C=0$ in $\mathcal{S}_{q}^{\prime}(M)$.

Definition 6.3. The (first) distributional basic cohomology (with real coefficients) of the measured foliations $\left.\mathcal{F}_{ \pm q}\right|_{M \backslash \Sigma_{q}}, \mathcal{F}_{ \pm q}$, denoted respectively by $H_{ \pm q}^{1}\left(M \backslash \Sigma_{q}, \mathbb{R}\right), H_{ \pm q}^{1}(M, \mathbb{R})$, are the images, under the cohomology map (6.2), of the spaces of basic currents $\mathcal{B}_{ \pm q}\left(M \backslash \Sigma_{q}\right), \mathcal{B}_{ \pm q}(M)$ :

$$
\begin{aligned}
H_{ \pm q}^{1}\left(M \backslash \Sigma_{q}, \mathbb{R}\right) & :=j_{q}\left(\mathcal{B}_{ \pm q}\left(M \backslash \Sigma_{q}\right)\right) \subset H^{1}\left(M \backslash \Sigma_{q}, \mathbb{R}\right) \\
H_{ \pm q}^{1}(M, \mathbb{R}) & :=j_{q}\left(\mathcal{B}_{ \pm q}(M)\right) \subset H^{1}(M, \mathbb{R})
\end{aligned}
$$

Basic currents in $\mathcal{B}_{q}(M)\left[\mathcal{B}_{-q}(M)\right]$ are related to the invariant distributions for the vector field $S[T]$, constructed in $[18, \S 4]$ as obstructions to the existence of smooth solutions to the cohomological equation $S u=f[T u=f]$. They therefore have a dynamical significance as they give obstructions to triviality of time changes for flows tangent to the foliation. In fact, time-change triviality is equivalent to the solvability of the cohomological equation [32, Def. 2.2.3 \& $\S 2.9]$.

Definition 6.4. A quasi $S$-invariant [a quasi $T$-invariant] distribution $\mathcal{D} \in \mathcal{D}^{\prime}(M \backslash \Sigma)$ is a distributional solution of the equation $S \mathcal{D}=0[T \mathcal{D}=0]$ in $\mathcal{D}^{\prime}(M \backslash \Sigma)$. An $S$-invariant [a $T$-invariant] distribution is a solution $\mathcal{D} \in \mathcal{S}_{q}^{\prime}(M)$ of the equation $S \mathcal{D}=0[T \mathcal{D}=0]$ in $\mathcal{S}_{q}^{\prime}(M)$. The vector space of quasi $S$-invariant [quasi $T$-invariant] distributions will be denoted by $\mathcal{I}_{q}\left(M \backslash \Sigma_{q}\right)$ $\left[\mathcal{I}_{-q}\left(M \backslash \Sigma_{q}\right)\right]$. The subspace of $S$-invariant [T-invariant] distributions will be denoted by $\mathcal{I}_{q}(M)\left[\mathcal{I}_{-q}(M)\right]$.

A distribution $\mathcal{D}$ will be considered as a current of degree 2 (and dimension 0 ). However, it is possible to identify distributions with currents of degree 0 (and dimension 2 ) by the isomorphism $\mathcal{D} \rightarrow \mathcal{D}^{*}$ determined by the area form $\omega_{q}$ on the (open) manifold $M \backslash \Sigma$ [56, Chap. IX, §2]. Given any distributions $\mathcal{D} \in \mathcal{D}^{\prime}\left(M \backslash \Sigma_{q}\right)$, let $\mathcal{D}^{*}$ be the current of degree 0 (and dimension 2) uniquely 
determined by the identity $\mathcal{D}=\mathcal{D}^{*} \omega_{q}$. It is then possible to define the (exterior) products $\mathcal{D}^{*} \eta_{S}$ or $\mathcal{D}^{*} \eta_{T}$ as in [11, p.42], [56, Chap. IX, §3]. Such products are the currents, of dimension (and degree) equal to 1 , given by the formulas

$$
\begin{aligned}
& \mathcal{D}^{*} \eta_{S}:=\mathcal{D}^{*} \wedge \eta_{S}=\imath_{S} \mathcal{D}, \\
& \mathcal{D}^{*} \eta_{T}:=\mathcal{D}^{*} \wedge \eta_{T}=-\imath_{T} \mathcal{D} .
\end{aligned}
$$

Lemma 6.5. The maps $C_{q}^{ \pm}: \mathcal{I}_{ \pm q}\left(M \backslash \Sigma_{q}\right) \rightarrow \mathcal{B}_{ \pm q}\left(M \backslash \Sigma_{q}\right)$ given by the formulas

$$
\begin{array}{ll}
C_{q}^{+}(\mathcal{D}):=\mathcal{D}^{*} \eta_{S}, & \mathcal{D} \in \mathcal{I}_{q}\left(M \backslash \Sigma_{q}\right), \\
C_{q}^{-}(\mathcal{D}):=\mathcal{D}^{*} \eta_{T}, & \mathcal{D} \in \mathcal{I}_{-q}\left(M \backslash \Sigma_{q}\right),
\end{array}
$$

are bijective. The inverse mappings are given by the formulas

$$
\begin{aligned}
& C^{+} \rightarrow-C^{+} \wedge \eta_{T} \in \mathcal{I}_{q}\left(M \backslash \Sigma_{q}\right), \\
& C^{-} \rightarrow C^{-} \wedge \eta_{S} \in \mathcal{I}_{-q}\left(M \backslash \Sigma_{q}\right) .
\end{aligned}
$$

In addition $C_{q}^{ \pm}(\mathcal{D}) \in \mathcal{B}_{ \pm q}(M) \subset \mathcal{B}_{ \pm q}\left(M \backslash \Sigma_{q}\right)$ if and only if $\mathcal{D} \in \mathcal{I}_{ \pm q}(M) \subset$ $\mathcal{I}_{ \pm q}\left(M \backslash \Sigma_{q}\right)$.

Proof. Let $X$ be a smooth vector field tangent to $\mathcal{F}_{q}\left[\mathcal{F}_{-q}\right]$. Then, since $\mathcal{F}_{ \pm q}$ are 1-dimensional, $X=W S[X=W T]$, where $W$ is a smooth function with compact support in $M \backslash \Sigma_{q}$ if $X$ has compact support and $W \in \Omega_{q}^{0}(M)$ if $X \in \mathcal{V}_{q}(M)$. Since, by $(6.6), \mathcal{D}^{*} \eta_{S}=\imath_{S} \mathcal{D}\left[\mathcal{D}^{*} \eta_{T}=-\imath_{T} \mathcal{D}\right]$ and $\imath_{S}^{2}=\imath_{T}^{2}=0$,

$$
\begin{gathered}
\imath_{X}\left(\mathcal{D}^{*} \eta_{S}\right)=W \imath_{S}\left(\mathcal{D}^{*} \eta_{S}\right)=W \imath_{S}^{2} \mathcal{D}=0 \\
{\left[\imath_{X}\left(\mathcal{D}^{*} \eta_{T}\right)=W \imath_{T}\left(\mathcal{D}^{*} \eta_{T}\right)=-W \imath_{T}^{2} \mathcal{D}=0\right],}
\end{gathered}
$$

in $\mathcal{D}^{\prime}(M \backslash \Sigma)$ if $\mathcal{D} \in \mathcal{D}^{\prime}(M \backslash \Sigma)$, in $\mathcal{S}_{q}^{\prime}(M)$ if $\mathcal{D} \in \mathcal{S}_{q}^{\prime}(M)$.

Since $\eta_{S}\left[\eta_{T}\right]$ is closed, if $\mathcal{D} \in \mathcal{I}_{q}\left(M \backslash \Sigma_{q}\right)\left[\mathcal{D} \in \mathcal{I}_{-q}\left(M \backslash \Sigma_{q}\right)\right]$, we have

$$
\begin{aligned}
d\left(\mathcal{D}^{*} \eta_{S}\right) & =d\left(\mathcal{D}^{*} \wedge \eta_{S}\right)=S \mathcal{D}=0 \\
{\left[d\left(\mathcal{D}^{*} \eta_{T}\right)\right.} & \left.=d\left(\mathcal{D}^{*} \wedge \eta_{T}\right)=-T \mathcal{D}=0\right]
\end{aligned}
$$

in $\mathcal{D}^{\prime}(M \backslash \Sigma)$. Hence, by $(6.4), \mathcal{D}^{*} \eta_{S} \in \mathcal{B}_{q}\left(M \backslash \Sigma_{q}\right)\left[\mathcal{D}^{*} \eta_{T} \in \mathcal{B}_{-q}\left(M \backslash \Sigma_{q}\right)\right]$. If $\mathcal{D} \in \mathcal{I}_{q}(M)\left[\mathcal{D} \in \mathcal{I}_{-q}(M)\right]$ the identities $\left(6.8^{\prime}\right)$ hold in $\mathcal{S}_{q}^{\prime}(M)$. Hence $\mathcal{D}^{*} \eta_{S} \in$ $\mathcal{B}_{q}(M)\left[\mathcal{D}^{*} \eta_{T} \in \mathcal{B}_{-q}(M)\right]$.

Conversely, let $C \in \mathcal{B}_{q}\left(M \backslash \Sigma_{q}\right)\left[C \in \mathcal{B}_{-q}\left(M \backslash \Sigma_{q}\right)\right]$. Let $\mathcal{D} \in \mathcal{D}^{\prime}(M \backslash \Sigma)$ be the distribution given by

$$
\begin{aligned}
\mathcal{D} & :=-C \wedge \eta_{T} \\
{[\mathcal{D}} & \left.:=C \wedge \eta_{S}\right] .
\end{aligned}
$$

Let $\phi$ be any real-valued function with compact support in $M \backslash \Sigma_{q}$ and let $X:=\phi S[X:=\phi T]$. Since $\imath_{X} \eta_{T}=\phi \imath_{S} \eta_{T}=\phi\left[\imath_{X} \eta_{S}=\phi \imath_{T} \eta_{S}=\phi\right]$ and 
$\imath_{X} C=0$,

$$
\begin{gathered}
\phi \mathcal{D}^{*} \eta_{S}=\imath_{X} \mathcal{D}=-\imath_{X}\left(C \wedge \eta_{T}\right)=\phi C \\
{\left[\phi \mathcal{D}^{*} \eta_{T}=-\imath_{X} \mathcal{D}=-\imath_{X}\left(C \wedge \eta_{S}\right)=\phi C\right],}
\end{gathered}
$$

hence $C=\mathcal{D}^{*} \eta_{S}\left[C=\mathcal{D}^{*} \eta_{T}\right]$ in $\mathcal{D}^{\prime}(M \backslash \Sigma)$. In addition, $\mathcal{D} \in \mathcal{I}_{q}\left(M \backslash \Sigma_{q}\right)$ $\left[\mathcal{D} \in \mathcal{I}_{-q}\left(M \backslash \Sigma_{q}\right)\right]$, since

$$
\begin{aligned}
S \mathcal{D} & =d\left(\mathcal{D}^{*} \eta_{S}\right)=d C=0 \\
{[T \mathcal{D}} & \left.=-d\left(\mathcal{D}^{*} \eta_{T}\right)=-d C=0\right]
\end{aligned}
$$

in $\mathcal{D}^{\prime}\left(M \backslash \Sigma_{q}\right)$. If $C \in \mathcal{B}_{q}(M)\left[C \in \mathcal{B}_{-q}(M)\right]$, since $S, T \in \mathcal{V}_{q}(M)$, the identities $\left(6.9^{\prime}\right)$, with $\phi \equiv 1$, and (6.10) hold in $\mathcal{S}_{q}^{\prime}(M)$. It follows that $\mathcal{D} \in \mathcal{I}_{q}(M)$ $\left[\mathcal{D} \in \mathcal{I}_{-q}(M)\right]$.

The space of all basic currents is filtered by a scale of subspaces of finite order currents, defined in terms of the weighted Sobolev spaces introduced in $[18, \S 2]$.

6.2. Weighted Sobolev spaces of currents. The space $L_{q}^{2}(M)$ is simply the space of square-summable complex-valued functions with respect to the area-form $\omega_{q}$ considered in $\S 2$, i.e. $L_{q}^{2}(M):=L^{2}\left(M, \omega_{q}\right)$. The space $H_{q}^{s}(M)$, $s \in \mathbb{N} \backslash\{0\}$, was defined in $[18, \S 2]$ as the completion of the space of smooth complex-valued functions $v$ on $M$ such that

$$
|v|_{s}:=\left(\sum_{i+j \leq s}\left|S^{i} T^{j} v\right|_{0}^{2}\right)^{1 / 2}<+\infty
$$

with respect to the norm $|\cdot|_{s}$. Let $H^{s}(M)$ denote the standard Sobolev spaces on the compact manifold $M$ [70, Chap. IV, §1]. The following properties hold:

(1) $L^{2}(M) \subset L_{q}^{2}(M)$

(2) $H^{1}(M) \equiv H_{q}^{1}(M)$,

(3) $H_{q}^{s}(M) \subset H^{s}(M), s \geq 2$.

The embedding (1) is an immediate consequence of the definitions, since $\omega_{q}$ is a smooth 2 -form on $M$, vanishing at $\Sigma$. The isomorphism in (2) can be proved by [18, Lemmas $2.1 \& 2.2]$. The proof is based on Poincaré's inequality and on the following formulas (see $[18,(2.7)]$ ):

$$
\begin{aligned}
& S=|z|^{-k}\left\{\Re\left(z^{k / 2}\right) \frac{\partial}{\partial x}-\Im\left(z^{k / 2}\right) \frac{\partial}{\partial y}\right\}, \\
& T=|z|^{-k}\left\{\Im\left(z^{k / 2}\right) \frac{\partial}{\partial x}+\Re\left(z^{k / 2}\right) \frac{\partial}{\partial y}\right\},
\end{aligned}
$$


where $z=x+i y$ is a canonical complex coordinate for the (orientable) quadratic differential $q$ at a zero $p \in \Sigma_{q}$ of even order $k$. By the same formulas and (2) one can also derive (3). We also remark that the space of smooth functions with compact support in $M \backslash \Sigma_{q}$ is not dense in $H_{q}^{s}(M)$ for $s \geq 2$, as a consequence of (3) and of trace theorems for Sobolev spaces [1, Th. 5.4]. However, it can be proved that $\Omega_{q}^{0} \subset H_{q}^{s}(M)$ is a dense subspace, for all $s \geq 0$. In fact, $C^{\infty}(M) \cap H_{q}^{s}(M)$ is dense in $H_{q}^{s}(M)$ by definition. The density of $\Omega_{q}^{0}$ in $C^{\infty}(M) \cap H_{q}^{s}(M)$ can be proved by Taylor expansion at each $p \in \Sigma_{q}$.

The weighted Sobolev spaces of 1 -forms, defined for all $s \geq 0$ by

$$
\mathcal{H}_{q}^{s}(M):=\left\{\alpha \in \mathcal{S}_{q}^{\prime}(M) \mid\left(\imath_{S} \alpha, \imath_{T} \alpha\right) \in H_{q}^{s}(M) \times H_{q}^{s}(M)\right\},
$$

have a natural Banach space structure, isomorphic to the product $H_{q}^{s}(M) \times$ $H_{q}^{s}(M)$. Since $\Omega_{q}^{0} \subset H_{q}^{s}(M)$ is dense, $\Omega_{q}^{1} \subset \mathcal{H}_{q}^{s}(M)$ is also dense, for all $s \geq 0$. The dual Sobolev spaces $H_{q}^{-s}(M), \mathcal{H}_{q}^{-s}(M)$ will be the distributional spaces defined, according to the standard Banach space theory, as the spaces of bounded functionals on $H_{q}^{s}(M), \mathcal{H}_{q}^{s}(M)$ respectively. Let $\mathcal{I}_{q}^{s}(M) \subset \mathcal{I}_{q}(M)$ $\left[\mathcal{I}_{-q}^{s}(M) \subset \mathcal{I}_{-q}(M)\right]$ be the space of $S$-invariant $[T$-invariant] distributions of order $s \in \mathbb{N}$, that is the space of $\mathcal{D} \in H_{q}^{-s}(M)$ such that $S \mathcal{D}=0[T \mathcal{D}=0]$ in $H_{q}^{-s-1}(M)$. Let $\mathcal{B}_{ \pm q}^{s}(M) \subset \mathcal{B}_{ \pm q}(M) \cap \mathcal{H}_{q}^{-s}(M)$ be the space of $\mathcal{F}_{ \pm q}$-basic currents of order $s \in \mathbb{N}$, defined as follows:

$$
\begin{aligned}
& \mathcal{B}_{q}^{s}(M):=\left\{C \mid \imath_{S} C=0 \in H_{q}^{-s}(M), \mathcal{L}_{S} C=0 \in \mathcal{H}_{q}^{-s-1}(M)\right\}, \\
& \mathcal{B}_{-q}^{s}(M):=\left\{C \mid \imath_{T} C=0 \in H_{q}^{-s}(M), \mathcal{L}_{T} C=0 \in \mathcal{H}_{q}^{-s-1}(M)\right\} .
\end{aligned}
$$

By Lemma 6.5, we have the following:

Lemma 6.6. A current $C \in \mathcal{B}_{q}^{s}(M)\left[C \in \mathcal{B}_{-q}^{s}(M)\right]$ if and only if the distribution $C \wedge \eta_{T} \in \mathcal{I}_{q}^{s}(M)\left[C \wedge \eta_{S} \in \mathcal{I}_{-q}^{s}(M)\right]$. In addition, the map

$$
\begin{gathered}
C \rightarrow-C \wedge \eta_{T}, \\
{\left[C \rightarrow C \wedge \eta_{S}\right],}
\end{gathered}
$$

is a bijection from $\mathcal{B}_{q}^{s}(M)\left[\mathcal{B}_{-q}^{s}(M)\right]$ onto the vector space $\mathcal{I}_{q}^{s}(M)\left[\mathcal{I}_{-q}^{s}(M)\right]$ of $S$-invariant $[T$-invariant $]$ distributions of order $s \in \mathbb{N}$.

Definition 6.7. The $H^{-s}$ (first) basic cohomology (with real coefficients) of the measured foliations $\mathcal{F}_{ \pm q}$ is given by

$$
H_{ \pm q}^{1, s}(M, \mathbb{R}):=j_{q}\left(\mathcal{B}_{ \pm q}^{s}(M)\right) \subset H_{ \pm q}^{1}(M, \mathbb{R}) \subset H^{1}(M, \mathbb{R})
$$

The $H^{-s}$ basic cohomology of the measured foliations $\mathcal{F}_{ \pm q}$ depends locally only on the A. Katok's fundamental class of the foliation. The proof is in fact based on Katok's local classification theorem for orientable measured foliations on surfaces, announced in [29, Th. 3], proved in [32, Th. 14.7.4] or [47, Th. 7.11.7]. 
Lemma 6.8. Let $q_{t} \in Q_{\kappa}, t \in[0,1]$, be a smooth family of quadratic differentials. Let $\Sigma_{t}$ be the set of zeroes of $q_{t}$. With the natural identification of the relative cohomology vector spaces $H^{1}\left(M, \Sigma_{t}, \mathbb{R}\right) \equiv H^{1}\left(M, \Sigma_{0}, \mathbb{R}\right)$, for all $t \in[0,1]$, we have:

$$
\begin{aligned}
{\left[\Im\left(q_{t}^{1 / 2}\right)\right] \equiv \lambda_{t}^{+}\left[\Im\left(q_{0}^{1 / 2}\right)\right] \in } & H^{1}\left(M, \Sigma_{0}, \mathbb{R}\right), \text { and } \\
& \text { all } \lambda_{t}^{+}>0 \Rightarrow H_{q_{t}}^{1, s}(M, \mathbb{R}) \equiv H_{q_{0}}^{1, s}(M, \mathbb{R}), \\
{\left[\Re\left(q_{t}^{1 / 2}\right)\right] \equiv \lambda_{t}^{-}\left[\Re\left(q_{0}^{1 / 2}\right)\right] \in } & H^{1}\left(M, \Sigma_{0}, \mathbb{R}\right), \text { and } \\
& \text { all } \lambda_{t}^{-}>0 \Rightarrow H_{-q_{t}}^{1, s}(M, \mathbb{R}) \equiv H_{-q_{0}}^{1, s}(M, \mathbb{R}) .
\end{aligned}
$$

Proof. Since quadratic differentials in $Q_{\kappa}$ are determined up to isotopies and the $H^{-s}$ basic cohomologies $H_{ \pm q}^{1, s}(M, \mathbb{R})$ are invariant under isotopies, we can assume that $\Sigma_{t} \equiv \Sigma_{0}$ and $q_{t} \equiv q_{0}$ on a neighbourhood of $\Sigma_{0}$. By a result of A. Katok [32, Th. 14.7.4] or [47, Th. 7.11.7], if $\left[\Im\left(q_{t}^{1 / 2}\right)\right]=\lambda_{t}^{+}\left[\Im\left(q_{0}^{1 / 2}\right)\right] \in$ $H^{1}\left(M, \Sigma_{0} ; \mathbb{R}\right)$ [if $\left.\left[\Re\left(q_{t}^{1 / 2}\right)\right]=\lambda_{t}^{-}\left[\Re\left(q_{0}^{1 / 2}\right)\right] \in H^{1}\left(M, \Sigma_{0} ; \mathbb{R}\right)\right]$, with $\lambda_{t}^{+}>0$ $\left[\lambda_{t}^{-}>0\right]$, for all $t \in[0,1]$, then the horizontal foliation $\mathcal{F}_{q_{t}}$ is isotopic to $\mathcal{F}_{q_{0}}$ [the vertical foliation $\mathcal{F}_{-q_{t}}$ is isotopic to $\mathcal{F}_{-q_{0}}$. In both cases the Katok's classification result holds since $q_{t} \equiv q_{0}$ in a neighborhood of the set $\Sigma_{t} \equiv \Sigma_{0}$ of common zeroes (hence the isotopy is equal to the identity on a neighbourhood of $\Sigma_{0}$ ). By the isotopy invariance of the $H^{-s}$ basic cohomology, the argument can be reduced to the case $\mathcal{F}_{q_{t}}=\mathcal{F}_{q_{0}}\left[\mathcal{F}_{-q_{t}}=\mathcal{F}_{-q_{0}}\right]$. Then, since the property that $q_{t} \equiv q_{0}$ in a neighbourhood of $\Sigma_{t} \equiv \Sigma_{0}$ implies that the dual Sobolev spaces of currents $\mathcal{H}_{q_{t}}^{-s}(M) \equiv \mathcal{H}_{q_{0}}^{-s}(M)$, by the definition $\left(6.12^{\prime}\right)$ of basic currents of order $s \in \mathbb{N}$, it follows that

$$
\begin{aligned}
\mathcal{B}_{q_{t}}^{s}(M) & =\mathcal{B}_{q_{0}}^{s}(M) \\
{\left[\mathcal{B}_{-q_{t}}^{s}(M)\right.} & \left.=\mathcal{B}_{-q_{0}}^{s}(M)\right] .
\end{aligned}
$$

In fact, let $\omega_{t}, \omega_{0}$ be the (degenerate) area-forms induced, respectively, by the quadratic differentials $q_{t}, q_{0}$. There exists a function $W_{t}: M \rightarrow \mathbb{R}^{+}$such that $W_{t} \equiv 1$ in a neighbourhood of $\Sigma_{0}$ and $\omega_{t}=W_{t} \omega_{0}$. Hence, $\mathcal{F}_{q_{t}}=\mathcal{F}_{q_{0}}$ $\left[\mathcal{F}_{-q_{t}}=\mathcal{F}_{-q_{0}}\right]$ implies $S_{t}=W_{t}^{-1} S_{0}\left[T_{t}=W_{t}^{-1} T_{0}\right]$. Then $\left(6.15^{\prime}\right)$ follows by applying the definition $\left(6.12^{\prime}\right)$. By $\left(6.15^{\prime}\right)$ and Definition 6.7 of the $H^{-s}$ basic cohomologies, the statement is proved.

6.3. A geometric estimate of the Poincaré constant. The property of weighted Sobolev spaces which is the most relevant to the content of this paper is a geometric estimate of the Poincaré constant (the constant in the Poincaré inequality for the Dirichlet form of the metric induced by a quadratic differential). Let $q \in Q_{\kappa}$. The Dirichlet form of the metric $R_{q}$, introduced in $[18,(2.6)]$, is defined as the hermitian form on the Hilbert space $H_{q}^{1}(M)$ given 
by

$$
\mathcal{Q}(u, v):=(S u, S v)_{q}+(T u, T v)_{q} .
$$

The following is a stronger version of [18, Lemma 2.2], which adds a crucial explicit geometric estimate of the constant.

LEMma 6.9. There is a constant $K_{g, \sigma}>0$ such that the following holds. Let $q \in Q_{g}^{(1)}$ be the square of a holomorphic differential, $\Sigma_{q}$ be the (finite) set of its zeroes and let $\sigma:=\operatorname{card}\left(\Sigma_{q}\right)$. Denote by $\|q\|$ the $R_{q}$-length of the shortest geodesic segment with endpoints in $\Sigma_{q}$. Then, for any $v \in H_{q}^{1}(M)$,

$$
\left|v-\int_{M} v \omega_{q}\right|_{0} \leq \frac{K_{g, \sigma}}{\|q\|} \mathcal{Q}(v, v)^{1 / 2}
$$

Proof. It was shown in $[18, \S 2]$ that many basic properties of the LaplaceBeltrami operator for a (non-degenerate) smooth Riemannian metric $R$ on a compact surface hold for the Dirichlet form $\mathcal{Q}$. In particular, since the MaxMin principle holds [18, Lemma 2.4], we have the following standard relation between the constant in the Poincaré lemma and the first non-zero eigenvalue $\lambda_{1}$ of $\mathcal{Q}$. Let $Z_{q}^{1} \subset H_{q}^{1}(M)$ be the subspace of zero average functions. Then

$$
\min _{v \in Z_{q}^{1}} \frac{\mathcal{Q}(v, v)}{|v|_{0}^{2}}=\lambda_{1}
$$

where the minimum is achieved at $v=u_{1}$, where $u_{1}$ is any eigenfunction with eigenvalue $\lambda_{1}$. A standard lower estimate for the first non-zero eigenvalue is given, for smooth Riemannian metrics on compact manifolds, in terms of the Cheeger isoperimetric constant [9]. Cheeger's argument [9], [4, Chap. III, D.4] carries over without modifications to the case of the degenerate Riemannian metric $R_{q}$. In fact, by [18, Th. 2.3 (iii)] all eigenfunctions of the Dirichlet form $\mathcal{Q}$ are smooth on $M$, including at $\Sigma_{q}$. In addition, the degenerate metric $R_{q}$ induces a well-defined smooth conformal structure on $M$, hence the key step of Cheeger's proof, the co-area formula [8, Th. 6.3], holds. Let $h_{q}$ be the Cheeger isoperimetric constant for the metric $R_{q}$,

$$
h_{q}:=\inf \frac{L_{q}(\Gamma)}{\min \left(\operatorname{vol}_{q}\left(M_{1}\right), \operatorname{vol}_{q}\left(M_{2}\right)\right)},
$$

where $L_{q}$ denotes the $R_{q}$-length and the infimum is taken over all compact 1-dimensional submanifolds $\Gamma \subset M$, dividing $M$ into two submanifolds with boundary $M_{1}, M_{2}$ such that $\partial M_{1}=\partial M_{2}=\Gamma$ and $M_{1} \cup M_{2}=M$. Then the Cheeger inequality reads

$$
\lambda_{1} \geq \frac{1}{4} h_{q}^{2} .
$$


The final step consists in a lower estimate of $h_{q}$. A 1-dimensional submanifold $\Gamma \subset M$ is a finite union of simple closed curves. If $\Gamma$ contains a closed curve $\gamma$

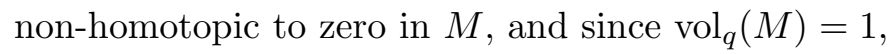

$$
\frac{L_{q}(\Gamma)}{\min \left(\operatorname{vol}_{q}\left(M_{1}\right), \operatorname{vol}_{q}\left(M_{2}\right)\right)} \geq L_{q}(\gamma) \geq\|q\|
$$

In fact, the shortest simple closed curve $\widehat{\gamma}$ freely homotopic to $\gamma$, which exists by [58, Th. 18.4], either contains singularities or it is a waist curve of an embedded flat cylinder. The boundary of the cylinder is then the union of a finite number of geodesic segments with endpoints in $\Sigma_{q}$. In either case $L_{q}(\gamma) \geq L_{q}(\widehat{\gamma}) \geq\|q\|$. If all simple closed curves in $\Gamma$ are homotopic to zero, since by an elementary lemma due to S. T. Yau [73, (1)-(4)] the infimum in the definition (6.18) can be equivalently taken on $\Gamma$ such that $M_{1}, M_{2}$ are connected, by the Jordan-Brouwer separation theorem, we can assume that $\Gamma$ is reduced to a single simple closed smooth curve bounding a simply connected domain $\Omega \subset M$. Let $p_{0} \in \Omega$ be any given point and consider the holomorphic $\operatorname{map} \Phi_{q}: \Omega \rightarrow \mathbb{C}$ given by

$$
\Phi_{q}(p):=\int_{p_{0}}^{p} q^{1 / 2}
$$

where $q^{1 / 2}$ is a holomorphic square root of $q$ on $\Omega$. The map $\Phi_{q}$ is well-defined and holomorphic since $\Omega$ is simply connected and $q^{1 / 2}$ is a holomorphic differential. By the open mapping theorem for holomorphic functions, $\Phi_{q}(\Omega) \subset \mathbb{C}$ is an open set and $\Phi_{q}$ is a branched covering with at most $2 g-1$ sheets by the Riemann-Hurwitz relation [13, I.2.7]. Since, by definition, the metric $R_{q}=\Phi_{q}^{*}\left(R_{e}\right)$, where $R_{e}$ is the Euclidean metric on $\mathbb{C}$,

$$
\frac{L_{q}(\Gamma)}{\min \left(\operatorname{vol}_{q}(\Omega), \operatorname{vol}_{q}(M \backslash \Omega)\right)} \geq \frac{L_{e}\left(\Phi_{q}(\Gamma)\right)}{\operatorname{vol}_{e}\left(\Phi_{q}(\Omega)\right)} \geq 2 \pi^{1 / 2}(2 g-1)^{1 / 2},
$$

where the last inequality follows from the classical isoperimetric inequality for the Euclidean metric in $\mathbb{R}^{2}$, that is $L_{e}^{2}(\partial D) \geq 4 \pi \operatorname{vol}_{e}(D)$ if $D \subset \mathbb{R}^{2}$ is any domain $[8, \S 6.2]$, and from the estimate $\operatorname{vol}_{e}\left(\Phi_{q}(\Omega)\right)=(2 g-1)^{-1} \operatorname{vol}_{q}(\Omega) \leq$ $(2 g-1)^{-1}$.

Let $d_{q}(M):=\max \left\{d_{q}\left(p, \Sigma_{q}\right) \mid p \in M\right\}$ be the maximum $R_{q}$-distance of any point of $M$ to a zero of $q$. Since $\|q\| / 2 \leq d_{q}(M)$ and, by [45, Cor. 5.6], there exists a constant $K_{g, \sigma}^{\prime}>0$ (depending only on $(g, \sigma)$ ) such that either $d_{q}(M) \leq \sqrt{2 / \pi}$ or $d_{q}(M) \leq K_{g, \sigma}^{\prime} /\|q\|$, the estimate $\left(6.16^{\prime}\right)$ follows from (6.17), $\left(6.18^{\prime}\right),(6.19)$ and $(6.21)$. 


\section{The structure of the space of basic currents of finite order}

The results we present below on the structure of the space of basic currents and on the basic cohomology of measured foliations are derived from [18] and therefore do not depend on the previous sections of the paper (with the exception of $\S 6.1$ and $\S 6.2)$.

7.1. Basic currents with non-vanishing cohomology class. Let $q \in Q_{\kappa}$, let $\Sigma:=\Sigma_{q}$ be finite set of its zeroes and $\mathcal{F}:=\mathcal{F}_{q}$ be its horizontal foliation. A current $C \in \mathcal{D}^{\prime}(M \backslash \Sigma)$ will be said to be of finite order $s \in \mathbb{N}$ if it can be extended to a continuous linear functional on the Sobolev space of forms $\mathcal{H}_{\text {loc }}^{s}\left(M \backslash \Sigma_{q}\right)$. The space of basic currents of order $s \in \mathbb{N}$ of the measured foliation $\left.\mathcal{F}\right|_{M \backslash \Sigma}$ will be denoted by $\mathcal{B}_{\mathcal{F}}^{s}(M \backslash \Sigma)$. The space $\mathcal{B}_{\mathcal{F}}^{s}(M):=\mathcal{B}_{q}^{s}(M)$ of basic currents of order $s \in \mathbb{N}$ for $\mathcal{F}=\mathcal{F}_{q}$ has been defined in Section 6.2. Let $j_{\Sigma}: \mathcal{Z}^{\prime}(M \backslash \Sigma) \rightarrow H^{1}(M \backslash \Sigma, \mathbb{R})$ be the cohomology map (6.2) and let

$$
\begin{aligned}
H_{\mathcal{F}}^{1, s}(M \backslash \Sigma, \mathbb{R}) & :=j_{\Sigma}\left(\mathcal{B}_{\mathcal{F}}^{s}(M \backslash \Sigma)\right) \subset H^{1}(M \backslash \Sigma, \mathbb{R}) \\
H_{\mathcal{F}}^{1, s}(M, \mathbb{R}) & :=j_{\Sigma}\left(\mathcal{B}_{\mathcal{F}}^{s}(M)\right) \subset H^{1}(M, \mathbb{R})
\end{aligned}
$$

be the $H^{-s}$ basic cohomologies of, respectively, $\left.\mathcal{F}\right|_{M \backslash \Sigma}$ and $\mathcal{F}$. We will prove the following:

TheOREM 7.1. There exists an integer $l>1$ such that the following holds. Let $q_{0} \in Q_{\kappa}$. For almost all $q \in \mathrm{SO}(2, \mathbb{R}) q_{0} \subset Q_{\kappa}$ with respect to the (Haar) Lebesgue measure, the basic cohomologies of order $s>l$ of the horizontal measured foliation $\mathcal{F}:=\mathcal{F}_{q}$ satisfy the following identities:

(i) $H_{\mathcal{F}}^{1, s}(M, \mathbb{R}) \equiv H_{\mathcal{F}}:=\left\{c \in H^{1}(M, \mathbb{R}) \mid c \wedge\left[\eta_{\mathcal{F}}\right]=0\right\}$,

(ii) $H_{\mathcal{F}}^{1, s}(M \backslash \Sigma, \mathbb{R}) \equiv H^{1}(M \backslash \Sigma, \mathbb{R})$.

The form $\eta_{\mathcal{F}}:=\Im\left(q^{1 / 2}\right)$ is the closed 1-form determined by the measured foliation $\mathcal{F}$, hence the cohomology class $\left[\eta_{\mathcal{F}}\right]$ is the projection under the map $H^{1}(M, \Sigma ; \mathbb{R}) \rightarrow H^{1}(M, \mathbb{R})$ of the Katok's fundamental class of $\mathcal{F}$.

Remark $7.1^{\prime}$. Let $\mu$ be a probability measure on the moduli space $\mathcal{M}_{\kappa}^{(1)}$ with the property that the conditional measures induced by $\mu$ on the orbits of the circle group $\mathrm{SO}(2, \mathbb{R})$ are absolutely continuous. By Theorem 7.1 and Fubini's theorem, the identities $\left(7.1^{\prime}\right)$ hold for $\mu$-almost all $q \in \mathcal{M}_{\kappa}^{(1)}$. The statement holds in particular if $\mu=\mu_{\kappa}^{(1)}$ is the (unique) absolutely continuous $G_{t}$-invariant probability measure on $\mathcal{M}_{\kappa}^{(1)}$.

In $[18, \S 4] S$-invariant distributions were constructed by the following method. Let $m^{+} \in \mathcal{M}_{q}^{+}$be a meromorphic function in $L_{q}^{2}(M)$ with poles at $\Sigma:=\Sigma_{q}$, the set of zeroes of a quadratic differential $q \in Q_{\kappa}$. If $U \in H_{q}^{-s}(M)$ 
is a solution of the cohomological equation $\mathrm{SU}=m^{+}$in $H_{q}^{-s-1}(M)$, then $\mathcal{D}=\partial_{q}^{+} U \in H_{q}^{-s-1}(M)$ is an $S$-invariant distribution on $M$. A refined version of this construction will enables us to detect the cohomology classes of basic currents corresponding to the $S$-invariant distributions constructed.

The spaces $\mathcal{M}_{\Sigma}^{ \pm}$of all meromorphic, respectively anti-meromorphic, functions with poles at $\Sigma:=\Sigma_{q}$ is a subspace of $\mathcal{S}_{q}^{\prime}(M)$. In fact, a function $m^{ \pm} \in \mathcal{M}_{\Sigma}^{ \pm}$can be regarded as a distribution in $H_{q}^{-s}(M)$ if and only if at any point $p \in \Sigma$ the order of a pole of $m^{ \pm}$does not exceed $k / 2+(k / 2+1) s$, where $k$ is the (even) order of zero of the quadratic differential $q$ at $p[18, \S 4]$. The distributions defined by $m^{ \pm} \in \mathcal{M}_{\Sigma}$ are given, for any function $v \in H_{q}^{s}(M)$, by the formula

$$
\left(m^{ \pm}, v\right):=\lim _{\epsilon \rightarrow 0} \int_{M \backslash \Sigma_{\epsilon}} m^{ \pm} v \omega_{q},
$$

where $\Sigma_{\epsilon}$ denotes the $\epsilon$-neighbourhood of the finite set $\Sigma$ with respect to the geodesic distance. The distribution defined by (7.2) is called the principal value of $m^{ \pm}$(principal values of meromorphic and anti-meromorphic functions on the complex plane and the action of the Cauchy-Riemann operators on distributions are studied in [3, $3.6 \& 3.8]$ ). There exist isomorphisms $h_{q}^{ \pm}: \mathcal{M}_{\Sigma}^{ \pm} \rightarrow \mathcal{H}_{\Sigma}^{ \pm}(M)$ onto the spaces of meromorphic, respectively anti-meromorphic, differentials with poles at $\Sigma$ :

$$
\begin{aligned}
& h_{q}^{+}\left(m^{+}\right):=m^{+} q^{1 / 2}, \\
& h_{q}^{-}\left(m^{-}\right):=m^{-} \bar{q}^{1 / 2} .
\end{aligned}
$$

In particular, $h_{q}^{ \pm}$maps the finite dimensional spaces $\mathcal{M}_{q}^{ \pm}$of $L_{q}^{2}(M)$ meromorphic, respectively anti-meromorphic, functions onto the spaces $\mathcal{H}_{q}^{ \pm}(M)$ of holomorphic, respectively anti-holomorphic, differentials on the Riemann surface $M_{q}$.

Let $m^{+} \in \mathcal{M}_{\Sigma}^{+}$be a meromorphic function with poles at $\Sigma$. Let $U \in$ $\mathcal{D}^{\prime}(M \backslash \Sigma)$ be a distributional solution of the equation $\mathrm{SU}=-\Re\left(m^{+}\right)$in $\mathcal{D}^{\prime}(M \backslash$ $\Sigma)$. Let $U^{*}$ be the (unique) current of dimension 2 and degree 0 such that $U=U^{*} \omega_{q}$. The 1-current $C \in \mathcal{D}^{\prime}(M \backslash \Sigma)$ uniquely determined by the identity

$$
d U^{*}=-\Re h_{q}^{+}\left(m^{+}\right)+C
$$

is basic for $\left.\mathcal{F}_{q}\right|_{M \backslash \Sigma}$. In fact, by (7.3), $C$ is closed (since $h_{q}^{+}\left(m^{+}\right)$is closed and $d U^{*}$ is exact) in $\mathcal{D}^{\prime}(M \backslash \Sigma)$ and $\imath_{S} C=0$, since $\mathrm{SU}=-\Re\left(m^{+}\right)$. Then $\mathcal{L}_{S} C=0$ by (6.4). A short computation also shows that $C=\mathcal{D}^{*} \eta_{S}$, where $\mathcal{D}$ is the quasi $S$-invariant distribution given by $\mathcal{D}=-C \wedge \eta_{T}=T U-\Im\left(\mathrm{m}^{+}\right)$. If $h_{q}^{+}\left(m^{+}\right)$is closed in $\mathcal{S}_{q}^{\prime}(M)$, which is the cases if $m^{+} \in L_{q}^{2}(M)$, and the equation $\mathrm{SU}=-\Re\left(m^{+}\right)$holds in $\mathcal{S}_{q}^{\prime}(M)$, then $C \in \mathcal{S}_{q}^{\prime}(M)$ and it is basic for $\mathcal{F}_{q}$. By (7.3), the cohomology class $[C]=\left[\Re h_{q}^{+}\left(m^{+}\right)\right] \in H^{1}(M \backslash \Sigma, \mathbb{R})$. 
It is a standard fact in the theory of Riemann surfaces, related to Hodge theory, that each cohomology class in $H^{1}\left(M_{q}, \mathbb{R}\right)$ can be represented by a harmonic differential $\Re\left(h^{+}\right)$, where $h^{+}$is a holomorphic differential on the Riemann surface $M_{q}$ [13, III.2]. Moreover, it follows from the Riemann-Roch theorem [13, III.4] that all cohomology classes in $H^{1}\left(M_{q} \backslash \Sigma ; \mathbb{R}\right)$ can be represented in the form $\Re\left(h^{+}\right)$where $h^{+}$is a meromorphic differential with at most simple poles at $\Sigma$. The strategy of our argument will therefore be based on the construction of solutions to the cohomological equation $\mathrm{SU}=-\Re\left(m^{+}\right)$ in $\mathcal{D}^{\prime}(M \backslash \Sigma)$ (in $\mathcal{S}_{q}^{\prime}(M)$ ) for appropriate choices of a meromorphic function $m^{+} \in \mathcal{M}_{\Sigma}^{+}$. The construction of basic currents depends therefore on the following:

Theorem 7.2 ([18, Th. 4.1]). There exists an integer $r>1$ such that the following holds. Let $q_{0} \in Q_{\kappa}$. For almost all $q \in \mathrm{SO}(2, \mathbb{R}) q_{0} \subset Q_{\kappa}$ with respect to the (Haar) Lebesgue measure, the cohomological equation $S u=f$ has a distributional solution $u \in H_{q}^{-r}(M)$ for any given $f \in H_{q}^{r-1}(M)$ with $\int_{M} f \omega_{q}=0$.

We would like to construct solutions of the equation $\mathrm{SU}=-\Re\left(m^{+}\right)$, as $h_{q}^{+}\left(m^{+}\right)$varies over all possible meromorphic differentials on $M$ with at most simple poles at $\Sigma$. Theorem 7.2 cannot be directly applied since $\Re\left(m^{+}\right) \notin$ $H_{q}^{r}(M)$. In the case of a smooth differential, which corresponds to the case that $m^{+} \in L_{q}^{2}(M)$, the idea is to first solve the equation locally around $\Sigma$.

Let $p \in \Sigma$ be a zero of $q$ of even order $k$. With respect to a canonical holomorphic coordinate $z=x+i y$ at $p, q(z)=z^{k} d z^{2}$. By $\left(6.11^{\prime}\right)$ we have:

$$
\begin{gathered}
S z=z^{-k / 2}, S \bar{z}=\bar{z}^{-k / 2}, \\
T z=\imath z^{-k / 2}, T \bar{z}=-\imath \bar{z}^{-k / 2} .
\end{gathered}
$$

By the identities (7.4), the equation $\mathrm{SU}=-\Re\left(m^{+}\right)$can be solved locally by expanding the meromorphic function $m^{+} \in L_{q}^{2}(M)$ in Laurent series around each point $p \in \Sigma$. In fact, if a $m^{ \pm} \in \mathcal{M}_{q}^{ \pm} \subset L_{q}^{2}(M)$, then $m^{ \pm}$has a pole of order at most $k / 2$ at a zero $p \in \Sigma$ of order $k$. Hence, by (7.4), the local solution $u^{ \pm}$of the equation $S u^{ \pm}=m^{ \pm}$, constructed formally by Laurent expansion, is respectively holomorphic, anti-holomorphic. Let $u_{0}$ be the function obtained as an extension to $M$ of the local solution of the equation $\mathrm{SU}=-\Re\left(m^{+}\right)$by partition of unity. Then $u_{0}$ is smooth on $M$, hence $u_{0} \in H_{q}^{1}(M)$, although $u_{0} \notin H_{q}^{2}(M)$, and $S u_{0}=-\Re\left(m^{+}\right)$in a neighbourhood of $\Sigma$. It follows that the function $f_{1}:=-\Re\left(m^{+}\right)-S u_{0} \in H_{q}^{s}(M)$, for all $s \in \mathbb{N}$. In fact, $f_{1}$ is smooth and has compact support in $M \backslash \Sigma$. In order to apply Theorem 7.2 to the equation $S u_{1}=f_{1}$ we have to impose the zero average condition:

$$
\int_{M} \Re\left(m^{+}+S u_{0}\right) \omega_{q}=\int_{M} \Re h_{q}^{+}\left(m^{+}\right) \wedge \eta_{S}=0 .
$$


Under condition (7.5) Theorem 7.2 yields a solution $u_{1} \in H_{q}^{-r}(M)$. Hence the distribution $U=u_{0}+u_{1} \in H_{q}^{-r}(M)$ is a solution of the equation $\mathrm{SU}=$ $-\Re\left(m^{+}\right)$in $H_{q}^{-r-1}(M)$ and the identity (7.3) determines a real $\mathcal{F}_{q^{-}}$basic current $C \in \mathcal{H}_{q}^{-r-1}(M)$, cohomologous to the harmonic form $\Re h_{q}^{+}\left(m^{+}\right)$. By this method, we can construct basic currents $C \in \mathcal{B}_{\mathcal{F}}^{r+1}(M)$ representing any given cohomology class in the subspace $\left\{c \in H^{1}(M, \mathbb{R}) \mid c \wedge\left[\eta_{\mathcal{F}}\right]=0\right\}$. In fact, such cohomology classes can be represented by smooth harmonic differentials on $M$, $\Re h_{q}^{+}\left(m^{+}\right), m^{+} \in L_{q}^{2}(M)$, satisfying the zero average condition (7.5).

Conversely, it can be proved that if $C \in \mathcal{B}_{\mathcal{F}}(M)$, then the zero average condition (7.5) holds. In fact,

$$
[C] \wedge\left[\eta_{\mathcal{F}}\right]=C \wedge \eta_{S}=\imath_{S} C \wedge \omega_{q}=0 .
$$

Theorem 7.1(i) is therefore proved.

In order to prove Theorem 7.1(ii) we need a regularization result proved in [18, Lemma 4.3B]. The main idea is to express all distributions in the dual weighted Sobolev spaces, which vanish on constant functions, as sums of iterated Cauchy-Riemann derivatives of smooth functions. In [18, Prop. 4.6A] we described the kernels of the Cauchy-Riemann operators $\partial_{-s}^{ \pm}: H_{q}^{-s+1}(M) \rightarrow$ $H_{q}^{-s}(M)$ modulo distributions supported at $\Sigma$. From this perspective such kernels are given by all equivalence classes of, respectively, meromorphic and anti-meromorphic, functions in $H_{q}^{-s+1}(M)$ modulo distributions supported at $\Sigma$. Such a conclusion follows immediately from the remark that $\partial^{ \pm}$are CauchyRiemann operators in the standard sense on the open manifold $M \backslash \Sigma$; hence their distributional kernel is given by, respectively, all holomorphic and antiholomorphic functions. We prove below a refined version of such a result:

Lemma 7.3. Let $\partial_{-s}^{ \pm}: H_{q}^{-s+1}(M) \rightarrow H_{q}^{-s}(M), s \geq 1$, denote the CauchyRiemann operators on dual weighted Sobolev spaces. The range $R\left(\partial_{-s}^{ \pm}\right) \subset$ $H_{q}^{-s}(M)$ of the Cauchy-Riemann operators is given by all distributions vanishing on constant functions. The kernel $N\left(\partial_{-s}^{ \pm}\right) \subset H_{q}^{-s+1}(M)$ consists respectively of meromorphic, anti-meromorphic, functions in $H_{q}^{-s+1}(M)$ which have no terms of the form $z^{-\ell(k / 2+1)}, \bar{z}^{-\ell(k / 2+1)}, \ell \in \mathbb{N} \backslash\{0\}$, in the Laurent expansion with respect to the canonical holomorphic coordinate, at a zero of $q$ of (even) order $k \in \mathbb{N} \backslash\{0\}$.

Proof. Let $\Phi \in H_{q}^{-s}(M)$. Then

$$
\left(U, \partial^{ \pm} v\right):=-(\Phi, v), \quad \text { for all } v \in H_{q}^{s}(M),
$$

defines a linear bounded functional on the (closed) range of the linear operator $\partial_{s}^{ \pm}: H_{q}^{s}(M) \rightarrow H_{q}^{s-1}(M)$. In fact, the kernel of $\partial^{ \pm}$on $H_{q}^{s}(M)$ contains only constant functions [18, Prop. 4.3A] and by hypothesis $\Phi$ vanishes on constant 
functions. In addition, by Poincaré's inequality (Lemma 6.9),

$$
\left|\left(U, \partial^{ \pm} v\right)\right|=|(\Phi, v)| \leq C|v|_{s} \leq C_{s}\left|\partial^{ \pm} v\right|_{s-1} .
$$

Finally, $U$ extends by the Hahn-Banach theorem to a linear bounded functional on $H_{q}^{s-1}(M)$, i.e. to a distribution $U \in H_{q}^{-s+1}(M)$, which by construction satisfies the equation $\partial_{-s}^{ \pm} U=\Phi$ in $H_{q}^{-s}(M)$. Hence the stated description of the range $R\left(\partial_{-s}^{ \pm}\right)$is proved (following [18, Prop. 4.6A]).

Let $U \in H_{q}^{-s+1}(M)$ be a solution of $\partial^{ \pm} U=0$. By the local properties of the Cauchy-Riemann operators on $M \backslash \Sigma$, the restriction of $U$ to $M \backslash \Sigma$ is a holomorphic, respectively an anti-holomorphic, function. Since $U \in H_{q}^{-s+1}(M)$, it is a meromorphic, respectively an anti-meromorphic, function with poles of order at most $k / 2+(k / 2+1)(s-1)$ at any point $p \in \Sigma$ where $q$ has a zero of (even) order $k$. A local computation of $\partial^{ \pm} U$ at points of $\Sigma$ concludes the proof. In fact, let $p \in \Sigma$ be a zero of order $k$ and let $v_{\alpha, \beta}$ be the Taylor coefficients of a smooth function $v$ supported in a neighbourhood of $p$, with respect to the complex coordinates $z, \bar{z}$, where $z$ is the canonical coordinate for $q$ at $p$ (determined by the conditions $q(z)=z^{k} d z^{2}$ and $z(p)=0$ ). Since $\partial^{ \pm}=S \pm i T$ and the vector fields $S, T$ are given by (7.4), with respect to the canonical coordinate, by applying Stokes theorem we get the following formulas:

$$
\begin{aligned}
& \left(\partial^{+}\left(z^{-n}\right), v\right)=-\lim _{\epsilon \rightarrow 0} \int_{M \backslash \Sigma_{\epsilon}} z^{-n} \partial^{+} v \omega_{q}=2 \pi v_{n-k / 2-1,0} \\
& \left(\partial^{-}\left(\bar{z}^{-n}\right), v\right)=-\lim _{\epsilon \rightarrow 0} \int_{M \backslash \Sigma_{\epsilon}} \bar{z}^{-n} \partial^{-} v \omega_{q}=2 \pi v_{0, n-k / 2-1} .
\end{aligned}
$$

The reader can compare (7.8) with similar formulas for the non-singular situation $[3, \S \S 3.6 \& 3.8]$. Since $U \in H_{q}^{-s+1}(M)$ and it is meromorphic, respectively anti-meromorphic, it can have at $p$ a pole of order at most $k / 2+(k / 2+1)(s-1)$. On the other hand, a smooth function $v \in H_{q}^{s}(M)$ is not allowed to have an arbitrary Taylor series at $p$. In fact, $v \in H_{q}^{s}(M)$ if and only if:

$$
v_{\alpha, \beta} \neq 0 \Longrightarrow \alpha+\beta \geq-k / 2+s(k / 2+1) \text { or } \alpha, \beta \in \mathbb{Z} \cdot(k / 2+1)
$$

By (7.8) and (7.9), if $n-k / 2-1=(\ell-1)(k / 2+1)$, that is $n=\ell(k / 2+1)$, $\ell \in \mathbb{N} \backslash\{0\}$,

$$
\begin{aligned}
& \partial^{+}\left(z^{-n}\right)=2 \pi \frac{(-1)^{\ell-1}}{(l-1) !}\left(\partial^{-}\right)^{\ell-1} \delta_{p} \neq 0, \\
& \partial^{-}\left(\bar{z}^{-n}\right)=2 \pi \frac{(-1)^{\ell-1}}{(l-1) !}\left(\partial^{+}\right)^{\ell-1} \delta_{p} \neq 0
\end{aligned}
$$


where $\delta_{p}$ is the Dirac delta at $p \in \Sigma$. If $n \leq k / 2+(k / 2+1)(s-1), z^{-n}$ and $\bar{z}^{-n} \in H_{q}^{-s+1}(M)$ locally and, since $\delta_{p} \in H^{-2}(M) \subset H_{q}^{-2}(M)$, the identities (7.10) hold in $H_{q}^{-s}(M)$. Such formulas can be compared with the analogous formulas for the derivatives of meromorphic and anti-meromorphic functions on the complex plane [3, Prop. 3.6.3].

We have therefore proved that, if $n \leq k / 2+(k / 2+1)(s-1)$ and $n=$ $\ell(k / 2+1)$ for any $\ell \geq 1$, then meromorphic and anti-meromorphic functions, with a pole of order $n$ at a zero of (even) order $k$ of the orientable holomorphic quadratic differential $q$, do not belong to the kernel $N\left(\partial_{-s}^{ \pm}\right) \subset H_{q}^{-s+1}(M)$. On the other hand, if $n \neq \ell(k / 2+1)$, then $\partial^{+}\left(z^{-n}\right)=\partial^{-}\left(\bar{z}^{-n}\right)=0$ in $H_{q}^{-s}(M)$, by (7.8) and (7.9), since $n-k / 2-1<-k / 2+s(k / 2+1)$.

By Lemma 7.3, if $\mathcal{D} \in H_{q}^{-s}(M)$ vanishes on constant functions, then there exist functions $u^{ \pm} \in L_{q}^{2}(M)$ (which can be taken of zero average) such that $\left(\partial^{ \pm}\right)^{s} u^{ \pm}=\mathcal{D}$. It is not possible in general to have a similar statement with $u^{ \pm}$smoother than $L_{q}^{2}$, since the operators $\partial^{ \pm}: H_{q}^{1}(M) \rightarrow L_{q}^{2}(M)$ have a nontrivial cokernel. However, the following result holds:

Lemma 7.4. Let $s \geq 0$ and $r \geq 1$. Then, any function $v \in H_{q}^{s}(M)$ such that $\int_{M} v \omega_{q}=0$ can be (non-uniquely) represented as follows:

$$
v=\sum_{i+j=r}\left(\partial^{+}\right)^{i}\left(\partial^{-}\right)^{j} v_{i j}, \quad \text { where } v_{i j} \in H_{q}^{s+r}(M) .
$$

The proof of this lemma is given in [18, Prop. 4.3A \& Lemma 4.3B] and will be omitted.

We can now prove a stronger version of Theorem 7.2:

COROLlary 7.5. There exists an integer $r>1$ (given by Theorem 7.2) such that the following holds. Let $q_{0} \in Q_{\kappa}$. For almost all $q \in \mathrm{SO}(2, \mathbb{R}) q_{0} \subset Q_{\kappa}$ with respect to the (Haar) Lebesgue measure, the cohomological equation $\mathrm{SU}=\Phi$ has a distributional solution $U \in H_{q}^{-s-2 r+1}(M)$ for any given $\Phi \in$ $H_{q}^{-s}(M)$ vanishing on constant functions.

Proof. Since $\Phi$ vanishes on constant functions, by iterated application of Lemma 7.3, there exists a zero average function $f \in L_{q}^{2}(M)$ such that

$$
\left(\partial^{+}\right)^{s} f=\Phi .
$$

Then, by applying Lemma 7.4, with $s=0$, to the function $f$, there exist zero average functions $f_{i j} \in H_{q}^{r-1}(M)$ such that

$$
\Phi=\left(\partial^{+}\right)^{s} f=\sum_{i+j=r-1}\left(\partial^{+}\right)^{i+s}\left(\partial^{-}\right)^{j} f_{i j} .
$$


By Theorem 7.2, it is possible to find distributional solutions $U_{i j} \in H_{q}^{-r}(M)$ of the equations $\mathrm{SU}_{i j}=f_{i j}$. Then

$$
U:=\sum_{i+j=r-1}\left(\partial^{+}\right)^{i+s}\left(\partial^{-}\right)^{j} U_{i j} \in H_{q}^{-2 r-s+1}(M)
$$

is a distributional solution of the equation $\mathrm{SU}=\Phi$ in $H_{q}^{-2 r-s}(M)$.

All cohomology classes $c \in H^{1}(M \backslash \Sigma, \mathbb{R})$ can be represented by the real part of a meromorphic differential $h^{+}$with at most simple poles at $\Sigma$. In fact, by the Riemann-Roch theorem [13, III.4.8] the complex dimension of the space of meromorphic differentials with at most simple poles at $\Sigma$ is equal to $2 g+\sigma-1$, where $\sigma:=\operatorname{card}(\Sigma)$, hence it is equal to the dimension of the cohomology $H^{1}(M \backslash \Sigma, \mathbb{C})$. If $h^{+}$has at most simple poles at $\Sigma$, then $h^{+}=h_{q}^{+}\left(m^{+}\right)$, where $m^{+} \in \mathcal{M}_{\Sigma}$ has a pole of order at most $k / 2+1$ at any zero of $q$ of order $k$. We would like to construct a solution in $\mathcal{D}^{\prime}(M \backslash \Sigma)$ of the equation $\mathrm{SU}=-\Re\left(\mathrm{m}^{+}\right)$. If $\Re\left(m^{+}\right)$does not vanish on constant functions, then no solution of finite order $U \in H_{q}^{-s}(M)$ exists. In fact, if $U \in H_{q}^{-s}(M)$, then $\mathrm{SU} \in H_{q}^{-s-1}(M)$ vanishes on constant functions. In order to bypass this difficulty, we construct a distributional solution of the modified equation $\mathrm{SU}=-\Re\left(m^{+}\right)+\delta$, where $\delta$ is a (Dirac) distribution supported on $\Sigma$ with the property that the distribution $\Phi:=-\Re\left(m^{+}\right)+\delta \in H_{q}^{-2}(M)$ vanishes on constant functions. The existence of a solution $U \in H_{q}^{-2 r-1}(M)$ is given by Corollary 7.5. Since $U$ is a solution of the modified equation $\mathrm{SU}=-\Re\left(m^{+}\right)+\delta$ in $H_{q}^{-2 r-2}(M)$ and $\delta$ is supported on $\Sigma$, the distribution $U$ solves the equation $\mathrm{SU}=-\Re\left(m^{+}\right)$in $\mathcal{D}^{\prime}(M \backslash \Sigma)$. Let $C \in \mathcal{H}_{q}^{-2 r-2}(M)$ be the currents obtained from $U$ by the identity (7.3). By construction of $U$ and (7.3), $C \in \mathcal{B}_{\mathcal{F}}^{s}(M \backslash \Sigma), s \geq 2 r+2$, and the cohomology class $[C]=\left[\Re\left(h^{+}\right)\right] \in H^{1}(M \backslash \Sigma, \mathbb{R})$. Theorem 7.1(ii) is therefore proved and the proof of Theorem 7.1 is completed.

7.2. Basic currents with vanishing cohomology class. Let $\mathcal{D}$ be a quasi $S$-invariant distribution and let $\mathcal{D}^{*}$ be the current of dimension 2 (and degree 0 ) corresponding to $\mathcal{D}$ under the isomorphism induced by $\omega_{q}$. Then $C:=d \mathcal{D}^{*} \in$ $\mathcal{B}_{\mathcal{F}}(M \backslash \Sigma)$. In fact,

$$
\begin{aligned}
\imath_{S} d \mathcal{D}^{*} & =\mathcal{L}_{S} \mathcal{D}^{*}=0 \\
\mathcal{L}_{S} d \mathcal{D}^{*} & =d \imath_{S} d \mathcal{D}^{*}=0
\end{aligned}
$$

in $\mathcal{D}^{\prime}(M \backslash \Sigma)$. Moreover, by its definition as a differential, $C$ has vanishing cohomology class. If $\mathcal{D}$ is $S$-invariant, then (7.15) holds in $\mathcal{S}_{q}^{\prime}(M)$ and $C \in \mathcal{B}_{\mathcal{F}}(M)$. It follows that the dimension of the vector space of basic currents with vanishing cohomology class is (at least) countable. In fact, it is possible to generate a sequence of linearly independent basic currents by iteration of the construction just explained. In other terms, if $\mathcal{D}$ is any (quasi) $S$-invariant distribution, 
then $\left\{T^{\ell} \mathcal{D}\right\}, \ell \in \mathbb{N}$, is a sequence of linearly independent (quasi) $S$-invariant distributions. We prove below that this procedure generates all basic currents for $\mathcal{F}_{M \backslash \Sigma}$ [for $\left.\mathcal{F}\right]$ (hence all quasi $S$-invariant [S-invariant] distributions) of finite order.

Lemma 7.6. Let $C$ be any real current of order $s \in \mathbb{N}$, dimension (and degree) equal to 1 , closed in $\mathcal{D}^{\prime}(M \backslash \Sigma)$. Then there exist a current $U^{*}$ of order $s-1$, dimension 2 (and degree 0 ) and a meromorphic differential $h^{+}$, with at most simple poles at $\Sigma$, such that

$$
d U^{*}=-\Re\left(h^{+}\right)+C
$$

in $\mathcal{D}^{\prime}(M \backslash \Sigma)$. If $C \in \mathcal{H}_{q}^{-s}(M)$ is closed in $\mathcal{S}_{q}^{\prime}(M)$, then there exist a current $U^{*} \in H_{q}^{-s+1}(M)$ and a differential $h^{+}$, holomorphic on $M_{q}$, such that (7.16) holds in $\mathcal{H}_{q}^{-s}(M)$.

Proof. If $C$ is closed in $\mathcal{D}^{\prime}(M \backslash \Sigma)$, since, by the Riemann-Roch theorem [13, III.4], any cohomology class in $H^{1}(M \backslash \Sigma ; \mathbb{R})$ can be represented as $\Re\left(h^{+}\right)$, where $h^{+}$is a meromorphic differential with at most simple poles at $\Sigma$, it follows from $\left[11, \S 15\right.$, Th. 12] that there exists a current $U^{*} \in \mathcal{D}^{\prime}(M \backslash \Sigma)$ of dimension 2 (and degree 0) such that (7.16) holds in $\mathcal{D}^{\prime}(M \backslash \Sigma)$. In addition, if $C$ is of order $s \in \mathbb{N}$, then $U^{*}$ is of order $s-1$ by (7.16).

If $C \in \mathcal{H}_{q}^{-s}(M)$ is closed in $\mathcal{S}_{q}^{\prime}(M)$, we cannot immediately deduce our statement from the standard de Rham theory. Instead, we argue as follows. Let $z$ be the canonical coordinate at a zero $p \in \Sigma$ of (even) order $k$ and let $\pi: \mathcal{U}_{p} \rightarrow D_{p}$ be the local covering map defined by $\pi_{p}(z):=z^{k / 2+1} /(k / 2+1)$ on a neighbourhood of $p \in M$ onto a neighbourhood $D_{p} \subset \mathbb{C}$ of the origin. The push-forward $\left(\pi_{p}\right)_{*}(C) \in \mathcal{D}^{\prime}\left(D_{p}\right)$ is well-defined (see [11, Chap. III, §11] or [56, Chap. IX, §4]) and closed, hence exact. There exists therefore a current $V_{p} \in \mathcal{D}^{\prime}\left(D_{p}\right)$ such that $d V_{p}=\left(\pi_{p}\right)_{*}(C)$. By the definition of the distributional space $\mathcal{S}_{q}^{\prime}(M)$, the pull-back $\pi_{p}^{*}\left(V_{p}\right)$ is also (locally) defined as a current in $\mathcal{S}_{q}^{\prime}(M)$ over $\mathcal{U}_{p}$. In fact, a form $\alpha \in \Omega_{q}^{2}(M)$, locally over $\mathcal{U}_{p}$, if and only if $\alpha=\pi_{p}^{*}\left(\lambda_{p}\right)$, where $\lambda_{p}$ is a (unique) smooth 2 -form on $D_{p}$. Then, by definition, $\pi_{p}^{*}\left(V_{p}\right)(\alpha):=V_{p}\left(\lambda_{p}\right)$. A computation shows that $d \pi_{p}^{*}\left(V_{p}\right)=C$ in $\mathcal{S}_{q}^{\prime}(M)$, locally over $\mathcal{U}_{p}$. Let $\left\{\mathcal{U}_{p}\right\}, p \in \Sigma$, be a finite family of disjoint open sets and, for each $p \in \Sigma$, let $\mathcal{U}_{p}^{\prime} \subset \subset \mathcal{U}_{p}$ be a relatively compact open subset such that $p \in \mathcal{U}_{p}^{\prime}$. Let $U_{p}^{*} \in \mathcal{S}_{q}^{\prime}(M)$ be a current such that $U_{p}^{*} \equiv \pi_{p}^{*}\left(V_{p}\right)$ in $\mathcal{U}_{p}^{\prime}$ and $U_{p}^{*} \equiv 0$ in $M \backslash \overline{\mathcal{U}}_{p}$. Then the current of dimension 2 (and degree 0 )

$$
U_{0}^{*}:=\sum_{p \in \Sigma} U_{p}^{*} \in \mathcal{S}_{q}^{\prime}(M)
$$

is such that $C-d U_{0}^{*} \in \mathcal{S}_{q}^{\prime}(M)$ has compact support in $M \backslash \Sigma$ and can therefore be extended to a current in $\mathcal{D}^{\prime}(M)$. In addition, since $C$ is closed and $d U_{0}^{*}$ 
is exact in $\mathcal{S}_{q}^{\prime}(M)$, by Lemma 6.2 the cohomology class $\left[C-d U_{0}^{*}\right]=[C] \in$ $H^{1}(M, \mathbb{R})$. By $[11, \S 15$, Th. 12] and by the standard representation theorem of cohomology classes on a Riemann surface [13, III.2], there exists a current $U_{1}^{*} \in \mathcal{D}^{\prime}(M)$ and a differential $h^{+}$, holomorphic on $M_{q}$, such that

$$
d U_{1}^{*}=-\Re\left(h^{+}\right)+C-d U_{0}^{*}
$$

in $\mathcal{D}^{\prime}(M)$. Let $U^{*}:=U_{0}^{*}+U_{1}^{*} \in \mathcal{S}_{q}^{\prime}(M)$. By $\left(7.17^{\prime}\right)$, the identity (7.16) holds in $\mathcal{S}_{q}^{\prime}(M)$. Since $C \in \mathcal{H}_{q}^{-s}(M), \Re\left(h^{+}\right)$is smooth on $M$ and $\Omega_{q}^{1}$ is dense in $\mathcal{H}_{q}^{s}(M)$, it follows that $d U^{*} \in \mathcal{H}_{q}^{-s}(M)$. Hence $U^{*} \in H_{q}^{-s+1}(M)$, and (7.16) holds in $\mathcal{H}_{q}^{-s}(M)$.

Let $\mathcal{F}:=\mathcal{F}_{q}$ be the horizontal foliation and $\Sigma:=\Sigma_{q}$ be the set of zeroes of a quadratic differential $q \in Q_{\kappa}$. We have:

TheOREM 7.7. Let $\mathcal{B}_{\mathcal{F}}^{s}(M \backslash \Sigma)\left[\mathcal{B}_{\mathcal{F}}^{s}(M)\right]$ be the vector space of all real basic currents of order $s \in \mathbb{N}$ for $\left.\mathcal{F}\right|_{M \backslash \Sigma}[$ for $\mathcal{F}]$. There exist exact sequences

$$
\begin{aligned}
0 & \rightarrow \mathbb{R} \rightarrow \mathcal{B}_{\mathcal{F}}^{s-1}(M \backslash \Sigma) \rightarrow \mathcal{B}_{\mathcal{F}}^{s}(M \backslash \Sigma) \rightarrow H_{\mathcal{F}}^{1, s}(M \backslash \Sigma ; \mathbb{R}), \\
0 & \rightarrow \mathbb{R} \rightarrow \mathcal{B}_{\mathcal{F}}^{s-1}(M) \rightarrow \mathcal{B}_{\mathcal{F}}^{s}(M) \rightarrow H_{\mathcal{F}}^{1, s}(M, \mathbb{R})
\end{aligned}
$$

Proof. Let $\delta_{s}: \mathcal{B}_{\mathcal{F}}^{s-1}(M \backslash \Sigma) \rightarrow \mathcal{B}_{\mathcal{F}}^{s}(M \backslash \Sigma)$ be the map defined as follows:

$$
\delta_{s}(C):=d U_{C}^{*}, \quad U_{C}:=-C \wedge \eta_{T} .
$$

The map $\left(7.18^{\prime}\right)$ is well-defined. In fact, if $C \in \mathcal{B}_{\mathcal{F}}^{s-1}(M \backslash \Sigma)\left[C \in \mathcal{B}_{\mathcal{F}}^{s-1}(M)\right]$, then $\delta_{s}(C)$ is by definition closed in $\mathcal{D}^{\prime}(M \backslash \Sigma)$ [in $\left.\mathcal{H}_{q}^{-s}(M)\right)$. Moreover, by Lemma 6.5, $U_{C}=-C \wedge \eta_{T}$ is a quasi $S$-invariant [an $S$-invariant] distribution of order $s-1$, hence $\imath_{S} d U_{C}^{*}=\mathcal{L}_{S} U_{C}^{*}=0$. It follows by $(6.4)$ that $\delta_{s}(C) \in$ $\mathcal{B}_{\mathcal{F}}^{s}(M \backslash \Sigma)\left[\delta_{s}(C) \in \mathcal{B}_{\mathcal{F}}^{s}(M)\right]$.

The image under $\delta_{s}$ of $\mathcal{B}_{\mathcal{F}}^{s-1}(M \backslash \Sigma)$ [of $\mathcal{B}_{\mathcal{F}}^{s-1}(M)$ ] consists of all basic currents $C^{s} \in \mathcal{B}_{\mathcal{F}}^{s}(M \backslash \Sigma)\left[C^{s} \in \mathcal{B}_{\mathcal{F}}^{s}(M)\right]$ with vanishing cohomology class. In fact, by Lemma 7.6, if $C^{s}$ has vanishing cohomology class, then there exists a distribution $U_{C}$ of order $s-1$ such that $d U_{C}^{*}=C^{s}$ in $\mathcal{D}^{\prime}(M \backslash \Sigma)$ [in $\mathcal{H}_{q}^{-s}(M)$ ]. Since $C^{s}$ is a basic current,

$$
\mathcal{L}_{S} U_{C}^{*}=\imath_{S}\left(d U_{C}^{*}\right)=\imath_{S} C^{s}=0
$$

in $\mathcal{D}^{\prime}(M \backslash \Sigma)$ [in $H_{q}^{-s}(M)$ ]. It follows that $U_{C}$ is a quasi $S$-invariant distribution [an $S$-invariant distribution] of order $s-1$, hence there exists a current $C^{s-1} \in$ $\mathcal{B}_{\mathcal{F}}^{s-1}(M \backslash \Sigma)\left[C^{s-1} \in \mathcal{B}_{\mathcal{F}}^{s-1}(M)\right]$ such that $U_{C}=-C^{s-1} \wedge \eta_{T}$. By $\left(7.18^{\prime}\right)$, $C^{s}=\delta_{s}\left(C^{s-1}\right)$.

The kernel of $\delta_{s}$ is given by the one-dimensional space $\mathbb{R} \cdot \eta_{S}$, i.e. by all basic currents which are scalar multiples of the closed 1-form $\eta_{S}$. In fact, if $\delta_{s}(C)=0$, then the distribution $U_{C}:=-C \wedge \eta_{T} \in \mathbb{R} \cdot \omega_{q}$. Since 
$C \in \mathcal{B}_{\mathcal{F}}^{s-1}(M \backslash \Sigma)\left[C \in \mathcal{B}_{\mathcal{F}}^{s-1}(M)\right]$, it follows that $C=\imath_{S} U_{C}$ in $\mathcal{D}^{\prime}(M \backslash \Sigma)$ [in $\left.\mathcal{H}_{q}^{-s+1}(M)\right]$, and hence $C \in \mathbb{R} \cdot \eta_{S}$. Let $\imath_{\mathcal{F}}: \mathbb{R} \rightarrow \mathcal{B}_{\mathcal{F}}(M \backslash \Sigma)$ be the linear map given by $\imath_{\mathcal{F}}(1):=\eta_{S}$. We have proved that the following sequences are exact:

$$
\begin{aligned}
0 & \rightarrow \mathbb{R} \underset{{ }_{{ }_{\mathcal{F}}}}{\longrightarrow} \mathcal{B}_{\mathcal{F}}^{s-1}(M \backslash \Sigma) \underset{\delta_{s}}{\longrightarrow} \mathcal{B}_{\mathcal{F}}^{s}(M \backslash \Sigma) \underset{j_{\Sigma}}{\longrightarrow} H_{\mathcal{F}}^{1, s}(M \backslash \Sigma ; \mathbb{R}), \\
0 & \rightarrow \mathbb{R} \underset{{ }_{l_{\mathcal{F}}}}{\longrightarrow} \mathcal{B}_{\mathcal{F}}^{s-1}(M) \underset{\delta_{s}}{\longrightarrow} \mathcal{B}_{\mathcal{F}}^{s}(M) \underset{j_{\Sigma}}{\longrightarrow} H_{\mathcal{F}}^{1, s}(M, \mathbb{R}) .
\end{aligned}
$$

The cohomology maps

$$
\begin{gathered}
\mathcal{B}_{\mathcal{F}}^{s}(M \backslash \Sigma) \underset{j_{\Sigma}}{\longrightarrow} H_{\mathcal{F}}^{1, s}(M \backslash \Sigma), \\
\mathcal{B}_{\mathcal{F}}^{s}(M) \underset{j_{\Sigma}}{\longrightarrow} H_{\mathcal{F}}^{1, s}(M),
\end{gathered}
$$

are surjective by definition.

\section{The non-uniform hyperbolicity of the Kontsevich-Zorich cocycle and an application to currents}

In Section 8.1 we will describe the invariant unstable and stable subbundles of the Kontsevich-Zorich cocycle in terms of the basic cohomology of the horizontal and vertical foliations of orientable quadratic differentials. This result is independent of the proof of the non-uniform hyperbolicity of the cocycle, concluded in Section 8.2. We then derive in Section 8.3 an Oseledectype theorem for the cocycle $G_{t}^{c}$ on the bundle $\mathcal{Z}_{\kappa}^{1}(M)$ of closed currents of order 1, introduced in Section 8.1.

8.1. The stable and unstable sub-bundles of the Kontsevich-Zorich cocycle as bundles of basic currents. In Section 7, we have obtained results on the $H^{-s}$ basic cohomology of measured foliations for $s \gg 1$. The stable and unstable sub-bundles of the Kontsevich-Zorich cocycle are related to the basic cohomology in the case $s=1$.

Let $H_{\kappa}^{-1}(M):=Q_{\kappa}^{(1)} \times H^{-1}(M) / \Gamma_{g}$, where the action of the mapping class group $\Gamma_{g}$ on the product is defined by pull-back on both factors. The mapping class group is a quotient of the group of orientation-preserving diffeomorphisms and the action of a diffeomorphism on currents by pull-back is described in [11, Chap. III, $\S 11]$ or [56, Chap. IX, $\S 5]$. The bundle $H_{\kappa}^{-1}(M)$ is the bundle of all distributions of order 1 on $M$ over the stratum $\mathcal{M}_{\kappa}^{(1)}$ of the moduli space. We introduce a cocycle $G_{t}^{0}$, over the Teichmüller geodesic flow $G_{t}$, defined on the Hilbert bundle $H_{\kappa}^{-1}(M)$ :

$$
G_{t}^{0}:=\left(G_{t} \times \mathrm{id}\right) / \Gamma_{g} \text { on } H_{\kappa}^{-1}(M):=\left(Q_{\kappa}^{(1)} \times H^{-1}(M)\right) / \Gamma_{g} .
$$


We also introduce a cocycle $G_{t}^{c}$ over the Teichmüller flow, defined on the Hilbert bundle $\mathcal{H}_{\kappa}^{-1}(M)$ of $H^{-1}$ currents over the stratum $\mathcal{M}_{\kappa}^{(1)}$. The fiber of the pullback of the bundle $\mathcal{H}_{\kappa}^{-1}(M)$ to the stratum $Q_{\kappa}^{(1)}$ of the Teichmüller space will be isomorphic by definition to the vector space $\mathcal{H}_{q}^{-1}(M)$ at any $q \in Q_{\kappa}^{(1)}$. Since the weighted Sobolev space of 1 -forms $\mathcal{H}_{q}^{1}(M)$ can be identified, by its definition (6.12), with the tensor product $\mathbb{R}^{2} \otimes H^{1}(M)$, the dual space $\mathcal{H}_{q}^{-1}(M)$ can be identified with the tensor product $\mathbb{R}^{2} \otimes H^{-1}(M)$. Let

$$
G_{t}^{c}:=\operatorname{diag}\left(e^{-t}, e^{t}\right) \otimes G_{t}^{0} \text { on } \mathcal{H}_{\kappa}^{-1}(M):=\mathbb{R}^{2} \otimes H_{\kappa}^{-1}(M) .
$$

The cocycle $G_{t}^{c}$ can be described as the cocycle obtained by parallel transport of currents, with respect to the trivial connection, along the orbits of the Teichmüller flow. In fact, by the formulas (2.7) describing the Teichmüller flow $G_{t}$ on $Q_{\kappa}^{(1)}$, we have:

$$
G_{t}^{c} \mid \mathcal{H}_{q}^{-1}(M) \equiv \mathrm{id}: \mathcal{H}_{q}^{-1}(M) \rightarrow \mathcal{H}_{G_{t}(q)}^{-1}(M) .
$$

Let $\mathcal{Z}_{\kappa}^{1}(M) \subset \mathcal{H}_{\kappa}^{-1}(M)$ be the infinite-dimensional sub-bundle over $\mathcal{M}_{\kappa}^{(1)}$ with fiber at $q \in \mathcal{M}_{\kappa}^{(1)}$ given by the vector space of closed currents of order 1 :

$$
\mathcal{Z}_{q}^{1}(M):=\mathcal{Z}_{q}^{\prime}(M) \cap \mathcal{H}_{q}^{-1}(M) .
$$

The sub-bundle $\mathcal{Z}_{\kappa}^{1}(M)$ is $G_{t}^{c}$-invariant and the cocycle induced by $G_{t}^{c}$ on the $H^{-1}$ de Rham cohomology bundle is isomorphic to the Kontsevich-Zorich cocycle. The latter is the essential motivation for the formulas (8.1) and (8.2) which define, respectively, the cocycles $G_{t}^{0}$ and $G_{t}^{c}$. Let

$$
j_{\kappa}: \mathcal{Z}_{\kappa}^{1}(M) \rightarrow \mathcal{H}_{\kappa}^{1}(M, \mathbb{R})
$$

be the natural bundle map, given fiber-wise by $\left(6.2^{\prime}\right)$, onto the cohomology bundle $\mathcal{H}_{\kappa}^{1}(M, \mathbb{R})$ (defined in $\S 1$ ).

Let $\mathcal{B}_{\kappa, \pm}^{1}(M) \subset \mathcal{Z}_{\kappa}^{1}(M)$ be the sub-bundles with fiber at $q \in \mathcal{M}_{\kappa}^{(1)}$ given by the vector spaces $\mathcal{B}_{ \pm q}^{1}(M)$ of $\mathcal{F}_{ \pm q}$-basic currents of order 1 , defined by $\left(6.12^{\prime}\right)$. Let

$$
\mathcal{B}_{\kappa}^{1}(M):=\mathcal{B}_{\kappa,+}^{1}(M)+\mathcal{B}_{\kappa,-}^{1}(M)
$$

Lemma 8.1. Let $\mu$ be any Borel probability ergodic invariant measure for the Teichmüller flow on a stratum $\mathcal{M}_{\kappa}^{(1)}$, with the property that the conditional measures induced by $\mu$ on the orbits of the action of the circle group $\mathrm{S}(2, \mathbb{R})$ on $\mathcal{M}_{\kappa}^{(1)}$ are absolutely continuous with respect to the (Haar) Lebesgue measure.

(1) The identity $j_{\kappa} \circ G_{t}^{c}=G_{t}^{K Z} \circ j_{\kappa}$ holds on $\mathcal{Z}_{\kappa}^{1}(M)$.

(2) The sub-bundles $\mathcal{B}_{\kappa, \pm}^{1}(M) \subset \mathcal{Z}_{\kappa}^{\prime}(M)$ are $G_{t}^{c}$-invariant, $\mu$-measurable and have $\mu$-almost everywhere constant (finite) rank. 
(3) The cocycle $G_{t}^{c}$ has $\mu$-almost everywhere strictly positive [strictly negative] Lyapunov spectrum on the invariant sub-bundle $\mathcal{B}_{\kappa,+}^{1}(M)\left[\mathcal{B}_{\kappa,-}^{1}(M)\right]$.

(4) The splitting (8.4) is direct and the restriction of the map $j_{\kappa}$ to the subbundle $\mathcal{B}_{\kappa}^{1}(M)$ is $\mu$-almost everywhere injective.

Proof. (1) It can be directly derived from the definition of the cocycles $G_{t}^{K Z}, G_{t}^{c}$ and of the cohomology map $j_{\kappa}$.

(2) The $G_{t}^{c}$-invariance of the sub-bundles $\mathcal{B}_{\kappa, \pm}^{1}(M)$ follows immediately from the definitions. We will write down in detail the proof of the measurability of the bundle $\mathcal{B}_{\kappa,+}^{1}(M)$. In fact, since $\mathcal{B}_{-q}^{1}(M)=\mathcal{B}_{r(q)}^{1}(M)$, where the diffeomorphism $r: \mathcal{M}_{\kappa}^{(1)} \rightarrow \mathcal{M}_{\kappa}^{(1)}$ is given by the action of the counterclockwise rotation of a right angle, the measurability of $\mathcal{B}_{\kappa,-}^{1}(M)$ then follows. By Lemma 6.6, the measurability of $\mathcal{B}_{\kappa,+}^{1}(M)$ can be reduced to the measurability of the bundle $\mathcal{I}_{\kappa}^{1}(M)$ with fiber at $q \in \mathcal{M}_{\kappa}^{(1)}$ given by the vector space $\mathcal{I}_{q}^{1}(M)$ of $S$-invariant distributions of order 1 . Let

$$
R_{q}^{1}(S):=\left\{S v \mid v \in H_{q}^{2}(M)\right\} \subset H^{1}(M) .
$$

We claim that any $q_{0} \in \mathcal{M}_{\kappa}^{(1)}$ has a neighborhood $\mathcal{U}_{0}$ such that the following holds. There exists a basis $\mathcal{V}(q):=\left\{v_{k}(q)\right\}, k \in \mathbb{N}$, of the vector space $H_{q}^{2}(M)$ with the property that, for all $k \in \mathbb{N}$, the mappings $q \rightarrow S v_{k}(q) \in H^{1}(M)$ are continuous on $\mathcal{U}_{0}$. In fact, there exists a neighbourhood $\widehat{\mathcal{U}}_{0}$ of $q_{0}$ in the space of quadratic differentials with the prescribed pattern of zeroes, endowed with the smooth topology, such that the following holds. Let $\Sigma_{0}$ be the set of zeroes of $q_{0}$. There exists on $\widehat{\mathcal{U}}_{0}$ a continuous mapping $q \rightarrow \phi_{q} \in \operatorname{Diff}_{0}^{+}(M)$ such that the set of zeroes $\Sigma_{\hat{q}}$ of the quadratic differential $\hat{q}:=\phi_{q}^{*}(q)$ coincides with $\Sigma_{0}$ and $\hat{q} \equiv q_{0}$ in a neighbourhood of $\Sigma_{0}$. By definition, the quadratic differentials $q$ and $\hat{q}$ yield the same point in Teichmüller space. The weighted Sobolev spaces $H_{\hat{q}}^{s}(M) \equiv H_{q_{0}}^{s}(M)$ (although they do not coincide as Hilbert spaces). Let $\mathcal{V}_{0}:=\left\{v_{k}\right\}, k \in \mathbb{N}$, be any basis of $H_{q_{0}}^{2}(M)$ and let $\mathcal{V}(q):=$ $\left\{v_{k}(q)\right\}$, where $v_{k}(q):=v_{k} \circ \phi_{q}^{-1}$, for all $k \in \mathbb{N}$. Then $\mathcal{V}(q)$ is a basis of $H_{q}^{2}(M)$ and the mappings $q \rightarrow S v_{k}(q) \in H^{1}(M)$ are continuous on $\widehat{\mathcal{U}}_{0}$. Let $\mathcal{U}_{0}:=\widehat{\mathcal{U}}_{0} / \operatorname{Diff}^{+}(M) \subset \mathcal{M}_{\kappa}^{(1)}$. The claim is therefore proved.

Let $v_{0} \equiv 1$ in the choice of the basis $\nu_{0}$. Let then $\mathcal{W}(q)=\left\{w_{k}(q)\right\}$ be the orthonormal system in the Hilbert space $H_{q}^{1}(M)$ constructed by applying the Gram-Schmidt orthonormalization procedure to the system $\left\{S v_{k} \mid k \in\right.$ $\mathbb{N} \backslash\{0\}\} \subset R_{q}^{1}(S)$. The system $\mathcal{W}(q)$ is well-defined at all $q \in \mathcal{U}_{0}$ such that $\mathcal{F}_{q}$ is quasi-minimal, since in that case the system $\left\{S v_{k} \mid k \in \mathbb{N} \backslash\{0\}\right\}$ is linearly independent. The subset of quadratic differentials with quasi-minimal horizontal foliation has full measure with respect to any Borel measure $\mu$ with purely continuous conditional measures on $\mu$-almost every orbit of the circle group. 
In fact, for any given $q$, the subset of the orbit $\mathrm{SO}(2, \mathbb{R}) \cdot q$ of the circle qroup with non-quasi-minimal horizontal foliation is countable as a consequence of the structure theorem for measured foliations (or area-preserving vector fields) on closed orientable surfaces [29], [77], [47, Th. 3.1.7]. It follows that $\mathcal{W}(q)$ is well-defined and the maps $q \rightarrow w_{k}(q) \in H^{1}(M)$ are continuous $\mu$-almost everywhere. The orthogonal projection $\pi_{q}: H_{q}^{1}(M) \rightarrow R_{q}^{1}(S)^{\perp}$, can be written as

$$
\pi_{q}(v)=v-\sum_{k=1}^{\infty}\left(v, w_{k}(q)\right)_{1} w_{k}(q), \quad v \in H^{1}(M),
$$

where $(\cdot, \cdot)_{1}$ is the inner product in $H_{q}^{1}(M)$. The subspace $R_{q}^{1}(S)^{\perp}$ depends measurably on $q \in \mathcal{U}_{0}$, since by (8.6) the orthogonal projection $\pi_{q}$ does. The space of continuous linear functionals on $H^{1}(M)$ vanishing on the closure of the subspace $R_{q}^{1}(S)$ is by definition the space $\mathcal{I}_{q}^{1}(M)$ of $S$-invariant distributions of order 1 . Since, by Lemma 6.4 , the vector space $\mathcal{B}_{q}^{1}(M)$ is canonically identified with $\mathcal{I}_{q}^{1}(M)$, the map $q \rightarrow \mathcal{B}_{q}^{1}(M)$ is $\mu$-measurable.

The almost everywhere finite dimensionality of $\mathcal{B}_{ \pm q}^{1}(M)$ is a consequence of the injectivity of the cohomology map $j_{q}: \mathcal{B}_{ \pm q}^{1}(M) \rightarrow H^{1}(M, \mathbb{R})$. By Theorem 7.7, if $C^{+} \in \mathcal{B}_{q}^{1}(M)\left[C^{-} \in \mathcal{B}_{-q}^{1}(M)\right]$ and $j_{q}\left(C^{ \pm}\right)=0$, then there exists $U^{ \pm} \in L_{q}^{2}(M)$ such that $\mathrm{SU}^{+}=0\left[T U^{-}=0\right]$ and $d U^{ \pm}=C^{ \pm}$. Since, by [35, Th. 2], $\mathcal{F}_{q}\left[\mathcal{F}_{-q}\right]$ is ergodic for $\mu$-almost all $q \in \mathcal{M}_{\kappa}^{(1)}$, it follows that $j_{q}\left(C^{ \pm}\right)=0$ implies $C^{ \pm}=0 \mu$-almost everywhere. Moreover, the dimension of $\mathcal{B}_{ \pm q}^{1}(M)$ is $\mu$-almost everywhere constant by its invariance under the Teichmüller flow $G_{t}$ and the ergodicity of the system $\left(G_{t}, \mu\right)$.

(3) The argument is based on the construction of appropriate infinitesimal Lyapunov functions in the sense of [31]. Let $q \in \mathcal{M}_{\kappa}^{(1)}, C^{ \pm} \in \mathcal{B}_{ \pm q}^{1}(M)$. Let $\mathcal{D}^{ \pm} \in H_{q}^{-1}(M), U^{ \pm} \in L_{q}^{2}(M)$ and $A^{ \pm} \in \mathbb{R}$ be defined as follows:

$$
\begin{array}{ll}
C^{+} \wedge \eta_{T}:=\mathcal{D}^{+}=\partial_{q}^{+} U^{+}+A^{+}, & U^{+} \perp \mathcal{M}_{q}^{+}, \\
C^{-} \wedge \eta_{S}:=\mathcal{D}^{-}=\partial_{q}^{-} U^{-}+A^{-}, & U^{-} \perp \mathcal{M}_{q}^{-},
\end{array}
$$

where $A^{ \pm}=\mathcal{D}^{ \pm}(1)$ and $U^{ \pm} \in L_{q}^{2}(M)$ are uniquely determined by the orthogonality conditions to the kernels $\mathcal{M}_{q}^{ \pm} \subset L_{q}^{2}(M)$ of the Cauchy-Riemann operators (consisting of meromorphic, respectively anti-meromorphic, functions). The existence of the functions $U^{ \pm} \in L_{q}^{2}(M)$ is given by the existence of distributional solutions of the equations $\partial^{ \pm} U=\mathcal{D}$ whenever $\mathcal{D} \in H_{q}^{-1}(M)$ and $\mathcal{D}(1)=0$ (Lemma 7.3 or $[18$, Prop. $4.6 \mathrm{~A}]$ ). Let $\mathcal{L}^{ \pm}: \mathcal{B}_{\kappa, \pm}^{1} \rightarrow \mathbb{R}$ be the non-negative functions defined by

$$
\mathcal{L}_{q}^{ \pm}\left(C^{ \pm}\right):=\left|U^{ \pm}\right|_{0}^{2}+\left(A^{ \pm}\right)^{2}
$$

Since the right-hand side of $\left(8.7^{\prime}\right)$ is invariant under the action of the mapping class group $\Gamma_{g}$, it defines a function on the measurable bundle $\mathcal{B}_{\kappa, \pm}^{1}(M)$. The 
functions $\mathcal{L}^{ \pm}$are measurable, since they extend to continuous functions on the smooth bundle $\mathcal{H}_{\kappa}^{-1}(M)$ with fiber $\mathcal{H}_{q}^{-1}(M)$ at all $q \in \mathcal{M}_{\kappa}^{(1)}$ and $\mathcal{B}_{\kappa, \pm}^{1}(M)$ are measurable sub-bundles by $(2)$. In addition, $\mathcal{L}_{q}^{ \pm}(\cdot)$ are continuous functions, homogeneous of degree 2 on each fiber and strictly positive on all non-zero $C^{ \pm} \in \mathcal{B}_{ \pm q}^{1}(M)$. We claim that the function $\mathcal{L}^{+}\left[\mathcal{L}^{-}\right]$is strictly increasing [decreasing] along the forward orbits of the cocycle $G_{t}^{c}$ if the horizontal foliation $\mathcal{F}_{q}$ [the vertical foliation $\mathcal{F}_{-q}$ ] is quasi-minimal.

By the formulas (2.7), $\left(2.7^{\prime}\right)$, which describe the evolution of a quadratic differential $q$ and of the corresponding Cauchy-Riemann operators $\partial_{q}^{ \pm}$under the Teichmüller flow $G_{t}$ on $Q_{\kappa}^{(1)}$, and by the definition of $G_{t}^{c}$, we have:

$$
\begin{aligned}
& \mathcal{D}_{t}^{+}=C^{+} \wedge \eta_{T}(t)=e^{t} C^{+} \wedge \eta_{T}=e^{t} \mathcal{D}_{0}^{+}, \\
& \mathcal{D}_{t}^{-}=C^{-} \wedge \eta_{S}(t)=e^{-t} C^{-} \wedge \eta_{S}=e^{-t} \mathcal{D}_{0}^{-} .
\end{aligned}
$$

Let $q_{t}:=G_{t}(q)$ and $\mathcal{M}_{t}^{ \pm}:=N\left(\partial_{t}^{ \pm}\right) \subset L_{q}^{2}(M)$, where $\partial_{t}^{ \pm}=\partial_{q}^{ \pm}$at $q=q_{t}$. Let $V_{t}^{ \pm} \in L_{q}^{2}(M)$ be the unique solutions of the equations

$$
\mathcal{D}_{0}^{ \pm}=\partial_{t}^{ \pm} V_{t}^{ \pm}+A_{0}^{ \pm}, \quad V_{t}^{ \pm} \perp \mathcal{M}_{t}^{ \pm} .
$$

We have

$$
\mathcal{D}_{t}^{ \pm}=e^{ \pm t} \mathcal{D}_{0}^{ \pm}=e^{ \pm t}\left\{\partial_{t}^{ \pm} V_{t}^{ \pm}+A_{0}^{ \pm}\right\}=\partial_{t}^{ \pm} U_{t}^{ \pm}+A_{t}^{ \pm},
$$

where $U_{t}^{ \pm}:=e^{ \pm t} V_{t}^{ \pm}$and $A_{t}^{ \pm}:=\mathcal{D}_{t}^{ \pm}(1)$. It follows finally from (8.7)-(8.10) that

$$
\mathcal{L}^{ \pm} \circ G_{t}^{c}\left(C^{ \pm}\right)=e^{ \pm 2 t}\left\{\left|V_{t}^{ \pm}\right|_{0}^{2}+\left(A_{0}^{ \pm}\right)^{2}\right\} .
$$

Let $\pi_{t}^{ \pm}: L_{q}^{2}(M) \rightarrow \mathcal{M}_{t}^{ \pm}$be the orthogonal projections. We claim that $V_{t}^{ \pm}$ is a solution of the following O.D.E. in $L_{q}^{2}(M)$ :

$$
\begin{aligned}
& u_{t}^{ \pm}=\partial_{t}^{ \pm} v_{t}^{ \pm}+\pi_{t}^{\mp}\left(u_{t}^{ \pm}\right), \\
& \frac{d}{d t} u_{t}^{ \pm}=\partial_{t}^{\mp} v_{t}^{ \pm}-M_{t}^{ \pm}\left(u_{t}^{ \pm}\right),
\end{aligned}
$$

where $M_{t}^{ \pm}:=M_{q}^{ \pm}$, at $q=q_{t}$, and $M_{q}^{ \pm}: L_{q}^{2}(M) \rightarrow \mathcal{M}_{q}^{ \pm}$is the linear operator defined as follows. Let $\left\{m_{1}^{ \pm}, \ldots, m_{g}^{ \pm}\right\}$be an orthonormal basis of $\mathcal{M}_{q}^{ \pm} \subset$ $L_{q}^{2}(M)$. Let $m_{i}^{ \pm}=\partial^{ \pm} v_{i}^{ \pm}+\pi_{q}^{\mp}\left(m_{i}^{ \pm}\right)$be the decompositions of the functions $m_{i}^{ \pm}$ according to the splitting (2.3). Then

$$
M_{q}^{ \pm}(u):=\sum_{i=1}^{g}\left(u, \partial^{ \pm} v_{i}^{ \pm}\right)_{q} m_{i}^{ \pm} .
$$

The equation (8.12) yields, as (2.12), an O.D.E. of the form $u^{\prime}=F(u, t)$ in the Banach space $L_{q}^{2}(M)$. In fact, its first line is just the Hilbert space orthogonal decomposition according to the splitting (2.3). Hence $F(t, u)$ is smooth and 
linear in the second variable. By the standard theorems on O.D.E.'s in Banach spaces [40, Chap. IV] the Cauchy problem for (8.12) has (locally) a unique solution for any initial data.

By the variational formulas (2.10) for the Cauchy-Riemann operators, any solution $u_{t}^{ \pm}$of (8.12) satisfies the identity $\frac{d}{d t}\left(\partial_{t}^{ \pm} u_{t}^{ \pm}\right) \equiv 0$ (no matter how the operator $M_{q}^{ \pm}$is defined) and, by the choice $\left(8.12^{\prime}\right)$ of $M_{q}^{ \pm}, u_{t}^{ \pm} \perp \mathcal{M}_{t}^{ \pm}$. It follows that $V_{t}^{ \pm}$is the unique solution $u_{t}^{ \pm}$of (8.12) satisfying the initial condition $u_{0}^{ \pm}=V_{0}^{ \pm}$.

Let $u_{t}^{ \pm}$be any solution of (8.12). Since $u_{t}^{ \pm} \perp \mathcal{M}_{t}^{ \pm}$, by (2.3) there exists a unique zero average function $w_{t}^{ \pm} \in H^{1}(M)$ such that $u_{t}^{ \pm}=\partial_{t}^{\mp} w_{t}^{ \pm}$. We claim that, if $\partial_{q}^{ \pm} u_{0}^{ \pm}$is a real-valued distribution, then $w_{t}^{ \pm}$is real-valued for all $t \in \mathbb{R}$. In fact, $\partial_{t}^{ \pm} u_{t}^{ \pm} \equiv \partial_{q}^{ \pm} u_{0}^{ \pm}$, which is real-valued, and $\partial_{t}^{ \pm} u_{t}^{ \pm}=\triangle_{t} w_{t}^{ \pm}$, where $\triangle_{t}:=\partial_{t}^{+} \partial_{t}^{-}$is the Laplace operator of the metric $R_{t}$ induced by the quadratic differential $q_{t}=G_{t}(q)$. Since the Laplacian is a real operator, it suffices to show that, for any $q \in Q_{\kappa}^{(1)}$, the unique zero average solution $w_{0} \in H_{q}^{1}(M)$ of the equation $\triangle_{q} w_{0}=0$ is $w_{0} \equiv 0$. In fact, $\left|\partial_{q}^{+} w_{0}\right|_{0}^{2}=-\left(\triangle_{q} w_{0}, \overline{w_{0}}\right)=0$, hence $w_{0}$ is a meromorphic function in $H_{q}^{1}(M)$, that is, by [18, Prop. 3.2], a constant function. The zero average condition then implies $w_{0} \equiv 0$. We can then compute:

$$
\begin{aligned}
\frac{d}{d t}\left|u_{t}^{ \pm}\right|_{0}^{2} & =2 \Re\left(u_{t}^{ \pm}, \frac{d}{d t} u_{t}^{ \pm}\right)_{q}=2 \Re\left(\partial_{t}^{\mp} w_{t}^{ \pm}, \partial_{t}^{\mp} v_{t}^{ \pm}\right)_{q} \\
& =2 \Re\left(\partial_{t}^{ \pm} w_{t}^{ \pm}, \partial_{t}^{ \pm} v_{t}^{ \pm}\right)_{q}=2 \Re\left(\partial_{t}^{ \pm} w_{t}^{ \pm}, \partial_{t}^{\mp} w_{t}^{ \pm}\right)_{q} \\
& =2 \Re\left\{\left|S_{t} w_{t}^{ \pm}\right|_{0}^{2}-\left|T_{t} w_{t}^{ \pm}\right|_{0}^{2} \pm 2 i\left(S_{t} w_{t}^{ \pm}, T_{t} w_{t}^{ \pm}\right)_{q}\right\} \\
& =2\left\{2\left|S_{t} w_{t}^{ \pm}\right|_{0}^{2}-\left|u_{t}^{ \pm}\right|_{0}^{2}\right\}=2\left\{\left|u_{t}^{ \pm}\right|_{0}^{2}-2\left|T_{t} w_{t}^{ \pm}\right|_{0}^{2}\right\} .
\end{aligned}
$$

We have exploited the fact that, since $w_{t}^{ \pm}$is real-valued, $\left(S_{t} w_{t}^{ \pm}, T_{t} w_{t}^{ \pm}\right)_{q} \in \mathbb{R}$. In addition, if $\partial_{q}^{ \pm} u_{0} \neq 0$ and $\mathcal{F}_{q}\left[\mathcal{F}_{-q}\right]$ is quasi-minimal; then $S_{t} w_{t}^{ \pm} \neq 0$ $\left[T_{t} w_{t}^{ \pm} \neq 0\right]$. In fact, otherwise, by the quasi-minimality property, $w_{t}^{ \pm}=0$ hence $u_{t}=0$ and $\partial^{ \pm} u_{0}^{ \pm} \equiv \partial_{t}^{ \pm} u_{t}^{ \pm}=0$.

Since the functions $V_{t}^{ \pm}$satisfy (8.12), we have by (8.11) and (8.13):

$$
\begin{aligned}
& \frac{d}{d t} \mathcal{L}^{+} \circ G_{t}^{c}\left(C^{+}\right)=e^{2 t}\left\{4\left|S_{t} w_{t}^{+}\right|_{0}^{2}+2\left(A_{0}^{+}\right)^{2}\right\}>0, \\
& \frac{d}{d t} \mathcal{L}^{-} \circ G_{t}^{c}\left(C^{-}\right)=-e^{-2 t}\left\{4\left|T_{t} w_{t}^{-}\right|_{0}^{2}+2\left(A_{0}^{-}\right)^{2}\right\}<0 .
\end{aligned}
$$

We have therefore constructed an infinitesimal Lyapunov function $\mathcal{L}^{+}\left[\mathcal{L}^{-}\right]$ on the finite-dimensional measurable bundle $\mathcal{B}_{\kappa,+}^{1}(M)\left[\mathcal{B}_{\kappa,-}^{1}(M)\right]$, strictly increasing [decreasing] along the Teichmüller orbit $G_{t}(q)$, for all $q \in \mathcal{M}_{\kappa}^{(1)}$ with quasi-minimal horizontal [vertical] foliation. The set of $q \in \mathcal{M}_{\kappa}^{(1)}$ with quasiminimal horizontal and vertical foliations has full $\mu$-measure. In fact, by the structure theorem for measured foliations (or area-preserving vector fields) on 
closed orientable surfaces, for any orbit of the circle group on $\mathcal{M}_{\kappa}^{(1)}$ the subset of quadratic differentials $q$ with non-quasi-minimal horizontal or vertical foliation is countable [29], [77], [47, Th. 3.1.7]. Since, by the definition $\left(8.7^{\prime}\right)$, $\mathcal{L}^{ \pm}\left(C^{ \pm}\right)>0$ if $C^{ \pm} \in \mathcal{B}_{\kappa, \pm}^{1} \backslash\{0\}$, by [31, Th. 2.1], the Lyapunov exponents of the cocycle $G_{t}^{c}$ restricted to the invariant bundle $\mathcal{B}_{\kappa,+}^{1}(M)\left[\mathcal{B}_{\kappa,-}^{1}(M)\right]$ are all strictly positive [strictly negative] $\mu$-almost everywhere.

(4) Let $q \in \mathcal{M}_{\kappa}^{(1)}$ be a quadratic differential with quasi-minimal horizontal and vertical foliations. Let $C^{ \pm} \in \mathcal{B}_{ \pm q}^{1}(M)$ be such that $j_{q}\left(C^{+}+C^{-}\right)=0$. Then the cohomology class $c=j_{q}\left(C^{+}\right)=-j_{q}\left(C^{-}\right) \in H^{1}\left(M_{q}, \mathbb{R}\right)$ belongs by (1) and (3) to both the stable and the unstable invariant bundle of the Kontsevich-Zorich cocycle, hence $c=0$. By the injectivity of the cohomology map $j_{\kappa}$ on $\mathcal{B}_{\kappa, \pm}^{1}(M)$ (proved above), it follows that $C^{+}=C^{-}=0$. We have therefore proved that $\mathcal{B}_{q}^{1}(M) \cap \mathcal{B}_{-q}^{1}(M)=\{0\}$ and that $j_{\kappa}$ is injective on $\mathcal{B}_{q}^{1}(M) \oplus \mathcal{B}_{-q}^{1}(M)$, for $\mu$-almost all $q \in \mathcal{M}_{\kappa}^{(1)}$.

By Lemma 8.1 every cohomology class $c \in H^{1}\left(M_{q}, \mathbb{R}\right)$, which can be represented by a current of order 1 , basic for the horizontal [vertical] foliation of a 'generic' quadratic differential $q \in \mathcal{M}_{\kappa}^{(1)}$, belongs to the unstable [stable] invariant sub-bundle of the Kontsevich-Zorich cocycle. In the remaining part of this section we will prove the converse statement. The argument is based on the geometric estimate of the Poincaré constant obtained in Lemma 6.9 and on the logarithmic law for geodesics in the moduli space due to H. Masur [44].

Let $E_{q}^{+}\left[E_{q}^{-}\right] \subset H^{1}\left(M_{q}, \mathbb{R}\right)$ be the (Lagrangian) subspaces corresponding to the subset of strictly positive [strictly negative] Lyapunov exponents in the Oseledec's decomposition of the Kontsevich-Zorich cocycle at an Oseledec regular point $q \in \mathcal{M}_{\kappa}^{(1)}$. By Oseledec's theorem [49], [32, Th. S.2.9] the set of regular points has full measure with respect to any ergodic $G_{t}$-invariant measure $\mu$ on $\mathcal{M}_{\kappa}^{(1)}$.

Lemma 8.2. Let $\mu$ be any ergodic $G_{t}$-invariant measure with the property that the conditional measures induced by $\mu$ on the orbits of the circle action are absolutely continuous. For $\mu$-almost all $q \in \mathcal{M}_{\kappa}^{(1)}$, every cohomology class $c^{ \pm} \in E_{q}^{ \pm}$can be represented by a current $C^{ \pm} \in \mathcal{B}_{ \pm q}^{1}(M)$. In addition, the following estimates holds. There exists a $\mu$-measurable function $K: \mathcal{M}_{\kappa}^{(1)} \rightarrow \mathbb{R}^{+}$ such that, if $c \in E_{q}^{+} \oplus E_{q}^{-}$has the harmonic representation $c=\left[\Re\left(m^{+} q^{1 / 2}\right)\right]$, $m^{+} \in \mathcal{M}_{q}^{+}$, then for a unique current $C \in \mathcal{B}_{+q}^{1}(M) \oplus \mathcal{B}_{-q}^{1}(M)$ and a unique zero average function $U \in L_{q}^{2}(M)$ we have:

$$
\begin{aligned}
& d U=C-\Re\left(m^{+} q^{1 / 2}\right), \\
& |U|_{0} \leq K(q)\|c\|_{q},
\end{aligned}
$$

where $\|c\|_{q}$ is the Hodge norm of $c \in H^{1}\left(M_{q}, \mathbb{R}\right)$. 
Proof. Let $q \in \mathcal{M}_{\kappa}^{(1)}$ be a regular point of the Kontsevich-Zorich cocycle, $c=c_{q}^{+}\left(m^{+}\right) \in E_{q}^{+}\left[E_{q}^{-}\right]$, where $m^{+} \in \mathcal{M}_{q}^{+}$is a meromorphic function and $c_{q}^{+}: \mathcal{M}_{q}^{+} \rightarrow H^{1}(M, \mathbb{R})$ is the isomorphism given by (2.4). By (2.5) the Hodge norm $\|c\|_{q}=\left|m^{+}\right|_{0}$. Let $c_{t}:=G_{t}^{K Z}(c)=\left[\Re\left(m_{t}^{+} q_{t}^{1 / 2}\right)\right]$, where $m_{t}^{+} \in \mathcal{M}_{t}^{+}$, the space of meromorphic functions with respect to the complex structure determined by the quadratic differential $q_{t}=G_{t}(q)$. Since the $L_{q}^{2}$ norm is invariant under the Teichmüller flow, there exist a $\mu$-measurable function $K_{1}(q)>0$ and an exponent $0<\lambda<1$ such that, if $c \in E_{q}^{+}\left[c \in E_{q}^{-}\right]$,

$$
\|c\|_{q_{t}}=\left|m_{t}^{+}\right|_{0} \leq K_{1}(q)\left|m^{+}\right|_{0} \exp (-\lambda|t|), \quad t \leq 0[t \geq 0] .
$$

Let $U_{t} \in L_{q}^{2}(M)$ be the unique function with zero average satisfying

$$
d U_{t}=c_{t}-c=\Re\left(m_{t}^{+} q_{t}^{1 / 2}\right)-\Re\left(m^{+} q^{1 / 2}\right) .
$$

By (2.12), (2.13) there exists a smooth one-parameter family of functions $v_{t} \in H^{1}(M)$ such that the function $U_{t}$ satisfies the following Cauchy problem in $L_{q}^{2}(M)$ :

$$
\begin{aligned}
\frac{d}{d t} U_{t} & =2 \Re\left(v_{t}\right), \\
U_{0} & =0 .
\end{aligned}
$$

If $v_{t}$ is chosen with zero average, since by definition the Dirichlet form $\mathcal{Q}(v, v)=$ $\left|\partial_{q}^{ \pm} v\right|_{0}^{2}$ for any orientable quadratic differential, by the Poincaré inequality (Lemma 6.9) and by the orthogonality of the decomposition in the first line of (2.12),

$$
\left|v_{t}\right|_{0} \leq K_{g, \sigma}\left\|q_{t}\right\|^{-1}\left|\partial_{t}^{+} v_{t}\right|_{0} \leq K_{g, \sigma}\left\|q_{t}\right\|^{-1}\left|m_{t}^{+}\right|_{0},
$$

where $\left\|q_{t}\right\|$ denotes the length of the shortest segment with endpoints in the set of zeroes of the quadratic differential $q_{t}$ with respect to the induced metric. By $\left(8.16^{\prime}\right)$ and (8.17), there exists a measurable function $K_{2}(q)>0$ such that, if $c \in E_{q}^{+}\left[c \in E_{q}^{-}\right]$,

$$
\left|\frac{d}{d t} U_{t}\right|_{0} \leq 2\left|v_{t}\right|_{0} \leq K_{2}(q)\left|m^{+}\right|_{0}\left\|q_{t}\right\|^{-1} \exp (-\lambda|t|), \quad t \leq 0[t \geq 0] .
$$

Since $U_{0}=0$, by Minkowski's integral inequality, (8.18) implies the following estimate:

$$
\left|U_{t}\right|_{0} \leq K_{2}(q)\left|m^{+}\right|_{0}\left|\int_{0}^{t} e^{-\lambda|s|}\left\|q_{s}\right\|^{-1} d s\right|, \quad t \leq 0[t \geq 0] .
$$

We claim that the integral in $\left(8.18^{\prime}\right)$ converges as $t \rightarrow-\infty[t \rightarrow+\infty]$. In [44] H. Masur proved a logarithmic law for the Teichmüller geodesic flow on the 
moduli space. The following estimate is the simplest step of Masur's argument [44, Prop. 1.2]:

$$
\limsup _{t \rightarrow \pm \infty} \frac{-\log \left\|q_{t}\right\|}{\log |t|} \leq \frac{1}{2}
$$

The statement holds for $\mu$-almost all quadratic differentials $q \in \mathcal{M}_{\kappa}^{(1)}$. It follows that our claim concerning $\left(8.18^{\prime}\right)$ holds $\mu$-almost everywhere. An alternative proof of the $\mu$-almost everywhere convergence of the integral in $\left(8.18^{\prime}\right)$ can be derived from a recent result of A. Eskin and H. Masur [12, Lemma 5.4], which strengthens a previous result by W. Veech [68, Th. 0.2], [69, Corollary 2.8], by applying integration by parts and Birkhoff's ergodic theorem. By $\left(8.18^{\prime}\right)$ and (8.19), there exists a $\mu$-measurable function $K_{3}(q)>0$ such that, if $c \in E_{q}^{+}$ $\left[c \in E_{q}^{-}\right]$

$$
\left|U_{t}\right|_{0} \leq K_{3}(q)\|c\|_{q}, \quad t \leq 0[t \geq 0]
$$

Let $U \in L_{q}^{2}(M)$ be any weak limit of $U_{t}$ as $t \rightarrow-\infty[t \rightarrow+\infty]$, which exists by (8.20) since all bounded subsets of the Hilbert space $L_{q}^{2}(M)$ are sequentially weakly compact. By contraction in (8.16),

$$
\begin{aligned}
\mathrm{SU}_{t} & =-\Re\left(m^{+}\right)+e^{t} \Re\left(m_{t}^{+}\right), \quad t \leq 0, \\
{\left[T U_{t}\right.} & \left.=\Im\left(m^{+}\right)-e^{-t} \Im\left(m_{t}^{+}\right), \quad t \geq 0\right],
\end{aligned}
$$

then by taking the limit as $t \rightarrow-\infty[t \rightarrow+\infty]$,

$$
\begin{aligned}
\mathrm{SU} & =-\Re\left(m^{+}\right), \\
{[T U} & \left.=\Im\left(m^{+}\right)\right] .
\end{aligned}
$$

The identities $\left(8.21^{\prime}\right)$ hold in the distributional space $H_{q}^{-1}(M)$. It follows that the current $C \in \mathcal{H}_{q}^{-1}(M)$ determined by the identity

$$
d U=C-\Re\left(m^{+} q^{1 / 2}\right)
$$

is a basic current of order 1 for the horizontal foliation $\mathcal{F}_{q}$ [for the vertical foliation $\left.\mathcal{F}_{-q}\right]$, representing the cohomology class $c \in E_{q}^{+}\left[c \in E_{q}^{-}\right]$. In fact, by $\left(8.21^{\prime}\right)$ and its definition (8.22), the current $C$ is closed and $\imath_{S} C=0\left[\imath_{T} C=0\right]$. Hence, by (6.4), $\mathcal{L}_{S} C=0\left[\mathcal{L}_{T} C=0\right]$. If the horizontal foliation $\mathcal{F}_{q}$ [the vertical foliation $\left.\mathcal{F}_{-q}\right]$ is ergodic (a property which holds for $\mu$-almost all $q \in \mathcal{M}_{\kappa}^{(1)}[35$, Th. 2]) the limit function $U \in L_{q}^{2}(M)$ is uniquely determined by $\left(8.21^{\prime}\right)$ and by the zero average condition. In addition, by (8.20), $U$ satisfies the estimate in (8.14). 
Since any cohomology class $c \in E_{q}^{+} \oplus E_{q}^{-}$can be split as $c=c^{+}+c^{-}$, where $c^{ \pm} \in E_{q}^{ \pm}$and $\left\|c^{ \pm}\right\|_{q} \leq \delta(q)\|c\|_{q}$, where $\delta(q)>0$ is the distorsion (in the sense of $\left[41\right.$, p. 269]) of the splitting $E_{q}^{+} \oplus E_{q}^{-}$, the cohomology map

$$
j_{q}: \mathcal{B}_{q}^{1}(M) \oplus \mathcal{B}_{-q}^{1}(M) \rightarrow E_{q}^{+} \oplus E_{q}^{-}
$$

is surjective and the estimate (8.14) holds, for $\mu$-almost all $q \in \mathcal{M}_{\kappa}^{(1)}$. The map (8.23) was proved to be $\mu$-almost everywhere injective in Lemma 8.1, (4).

Remark $8.2^{\prime}$. Lemma 8.2 can be viewed as a regularity result. In fact, by Theorem 7.1(i), for almost all $q \in \mathcal{M}_{\kappa}^{(1)}$, every cohomology class $c \in H_{q}:=$ $\left\{c \in H^{1}\left(M_{q}, \mathbb{R}\right) \mid c \wedge\left[\Im\left(q^{1 / 2}\right)\right]=0\right\}$, can be represented by a basic current $C \in \mathcal{B}_{q}(M)$ of finite order $l>1$. The subspace $H_{q}$ has codimension 1 , hence it has dimension $2 g-1$. By Lemma 8.2 and Theorem 8.5 below, if $c$ belongs to a Lagrangian subspace $E_{q}^{+} \subset H_{q}$ (which has dimension equal to $g \geq 2$ ), then $C$ actually has order 1 .

We can finally derive from Lemma 8.1 and Lemma 8.2 the following characterization of the invariant unstable [stable] bundle $E_{\kappa}^{+}\left[E_{\kappa}^{-}\right]$of the KontsevichZorich cocycle on any stratum $\mathcal{M}_{\kappa}^{(1)}$.

THEOREM 8.3. Let $\mu$ be any ergodic $G_{t}$-invariant Borel probability measure on $\mathcal{M}_{\kappa}^{(1)}$ with the property that the conditional measures induced on the orbits of the circle action are absolutely continuous. The invariant unstable [stable] sub-bundle of the Kontsevich-Zorich cocycle coincides $\mu$-almost everywhere with the bundle of $H^{-1}$ basic cohomologies for the horizontal [vertical] foliation of quadratic differentials:

$$
\begin{aligned}
& E_{\kappa}^{+}=j_{\kappa}\left(\mathcal{B}_{\kappa,+}^{1}(M)\right):=\mathcal{H}_{\kappa,+}^{1,1}(M, \mathbb{R}), \\
& E_{\kappa}^{-}=j_{\kappa}\left(\mathcal{B}_{\kappa,-}^{1}(M)\right):=\mathcal{H}_{\kappa,-}^{1,1}(M, \mathbb{R}) .
\end{aligned}
$$

Theorem 8.3 implies that the unstable subspace $E_{q}^{+}$[the stable subspace $E_{q}^{-}$] depends locally only on the fundamental class of the horizontal measured foliation $\mathcal{F}_{q}$ [of the vertical measured foliation $\mathcal{F}_{-q}$ ], a property conjectured in [38, Th. p. 8, (3)]. In fact, by Lemma 6.8 and Theorem 8.3, we have:

COROLlary 8.3'. For almost all (regular) points $q_{0} \in \mathcal{M}_{\kappa}^{(1)}$ of the Kontsevich-Zorich coycle, there exists a neighbourhood $\mathcal{U}_{0} \subset \mathcal{M}_{\kappa}^{(1)}$ with the property that $q_{0} \in \mathcal{U}_{0}$ and, for almost all (regular) points $q \in \mathcal{U}_{0}$,

$$
\begin{aligned}
& {\left[\Im\left(q^{1 / 2}\right)\right]=\left[\Im\left(q_{0}^{1 / 2}\right)\right] \in H^{1}\left(M, \Sigma_{\kappa}, \mathbb{R}\right) \Longrightarrow E_{q}^{+}=E_{0}^{+},} \\
& {\left[\Re\left(q^{1 / 2}\right)\right]=\left[\Re\left(q_{0}^{1 / 2}\right)\right] \in H^{1}\left(M, \Sigma_{\kappa}, \mathbb{R}\right) \Longrightarrow E_{q}^{-}=E_{0}^{-},}
\end{aligned}
$$

where $E_{q}^{ \pm}\left[E_{0}^{ \pm}\right]$denotes the invariant unstable, respectively stable, subspace of the Kontsevich-Zorich cocycle at $q\left[q_{0}\right]$. 
8.2. The Kontsevich-Zorich cocycle is non-uniformly hyperbolic.

LEMMA 8.4. The set of quadratic differentials $q_{0}$ having the following properties is dense in $\mathcal{M}_{\kappa}^{(1)}$ :

(1) The horizontal foliation $\mathcal{F}_{q_{0}}$ is Lagrangian (Definition 4.3);

(2) Any neighbourhood $\mathcal{U}_{\kappa}^{(1)} \subset \mathcal{M}_{\kappa}^{(1)}$ of $q_{0}$ contains a compact positive measure set $\mathcal{P}_{\kappa}^{(1)}$ such that

(a) all $q \in \mathcal{P}_{\kappa}^{(1)}$ are Birkhoff generic points for the Teichmüller flow and Oseledec regular points for the Kontsevich-Zorich cocycle;

(b) the stable and the unstable subspace $E_{q}^{ \pm}$of the Kontsevich-Zorich cocycle depend continuously on $q \in \mathcal{P}_{\kappa}^{(1)}$;

(c) the Poincaré dual $P\left(\mathcal{L}_{0}\right)$ of the Lagrangian subspace $\mathcal{L}_{0}:=\mathcal{L}\left(\mathcal{F}_{q_{0}}\right)$, generated by the regular trajectories of $\mathcal{F}_{q_{0}}$, is transverse to $E_{q}^{-}$for all $q \in \mathcal{P}_{\kappa}^{(1)}$.

Proof. Let $\mathcal{F}_{\kappa}(M)$ be the set of all isotopy equivalence classes of orientable measured foliations $\mathcal{F}$ on $M$ with a finite set of canonical saddle-like singularities of multiplicities $\kappa:=\left(k_{1}, \ldots, k_{\sigma}\right)$. Let $\mathcal{F}_{\kappa}^{\prime}(M) \subset \mathcal{F}_{\kappa}(M)$ be the subset given by the measured foliations $\mathcal{F}$ such that there exists $q \in Q_{\kappa}$ with $\mathcal{F}_{q}=\mathcal{F}$ (or $\mathcal{F}_{-q}=\mathcal{F}$ ). The space $Q_{\kappa}$ has locally a product structure modeled on $\mathcal{F}_{\kappa}^{\prime}(M) \times \mathcal{F}_{\kappa}^{\prime}(M)$. Let $\mathcal{U}_{\kappa}=\mathcal{U}_{\kappa}^{+} \times \mathcal{U}_{\kappa}^{-}, \mathcal{U}_{\kappa}^{ \pm} \subset \mathcal{F}_{\kappa}^{\prime}(M)$, be any open subset of $Q_{\kappa}$ having a product structure. By Luzin's theorem, there exists a compact set of positive measure $\mathcal{P}_{\kappa}^{-} \subset \mathcal{U}_{\kappa}^{-}$such that the basic cohomology map $\mathcal{F} \rightarrow H_{\mathcal{F}}^{1,1}(M, \mathbb{R})$ is continuous on $\mathcal{P}_{\kappa}^{-}$. Let $\mathcal{F}_{0}^{-}$be a density point of $\mathcal{P}_{\kappa}^{-}$ and let $E_{0}^{-}=H_{\mathcal{F}_{0}^{-}}^{1,1}(M, \mathbb{R})$. By Lemma 4.4, there exists a Lagrangian foliation $\mathcal{F}_{0}^{+} \in \mathcal{U}_{\kappa}^{+}$such that the Poincaré dual $P\left(\mathcal{L}_{0}\right)$ of the Lagrangian subspace $\mathcal{L}_{0}:=\mathcal{L}\left(\mathcal{F}_{0}^{+}\right)$is transverse to $E_{0}^{-}$. Let $q_{0} \in \mathbb{R}^{+} \cdot \mathcal{U}_{\kappa}$ be the quadratic differential uniquely defined by the condition $\left(\mathcal{F}_{q_{0}}, \mathcal{F}_{-q_{0}}\right) \in \mathbb{R}^{+} \cdot\left(\mathcal{F}_{0}^{+}, \mathcal{F}_{0}^{-}\right)$and the condition that the total area $A\left(q_{0}\right)=1$.

Let $\mathcal{U}_{\kappa}^{(1)} \subset \mathcal{M}_{\kappa}^{(1)}$ be a neighbourhood of $q_{0}$. By construction and Theorem 8.3, the map $q \rightarrow E_{q}^{-}$is defined and continuous on a positive measure subset of $\mathcal{U}_{\kappa}^{(1)}$ having density 1 at $q_{0}$ and the subspace $P\left(\mathcal{L}_{0}\right)$ is transverse to $E_{q_{0}}^{-}$. Hence, by Birkhoff's ergodic theorem, Oseledec's theorem and Luzin's theorem, there exists a compact set of positive measure $\mathcal{P}_{\kappa}^{(1)} \subset \mathcal{U}_{\kappa}^{(1)}$, consisting of quadratic differentials that are Birkhoff generic points for the Teichmüller flow and Oseledec regular points for the Kontsevich-Zorich coycle, such that the maps $q \rightarrow E_{q}^{ \pm}$are continuous and $P\left(\mathcal{L}_{0}\right)$ is transverse to $E_{q}^{-}$, for all $q \in \mathcal{P}_{\kappa}^{(1)}$.

TheOREM 8.5. The Kontsevich-Zorich cocycle is non-uniformly hyperbolic on every connected component $\mathcal{C}_{\kappa}^{(1)}$ of a stratum $\mathcal{M}_{\kappa}^{(1)}$. In fact, the Lya- 
punov exponents of the normalized restriction of the absolutely continuous invariant measure $\mu_{\kappa}^{(1)}$ to $\mathcal{C}_{\kappa}^{(1)}$ form a symmetric subset of the real line and satisfy the following inequalities:

$$
\begin{aligned}
1=\lambda_{1}>\lambda_{2} \geq & \cdots \geq \lambda_{g}>0>\lambda_{g+1}=-\lambda_{g} \geq \cdots \\
& \cdots \geq \lambda_{2 g-1}=-\lambda_{2}>\lambda_{2 g}=-\lambda_{1}=-1 .
\end{aligned}
$$

Proof. The argument will proceed by contradiction. If (8.26) does not hold on a connected component $\mathcal{C}_{\kappa}^{(1)} \subset \mathcal{M}_{\kappa}^{(1)}$, there exists a natural number $k<g$ such that $\lambda_{k} \neq 0$ and $\lambda_{k+1}=0$. Let $E_{k}^{+} \subset \mathcal{H}_{\kappa}^{1}(M, \mathbb{R})$ be the $k$-dimensional unstable invariant sub-bundle of the Kontsevich-Zorich cocycle, which corresponds to the set of strictly positive Lyapunov exponents $\left\{\lambda_{1}, \ldots, \lambda_{k}\right\}$ in the Oseledec's decomposition. By Corollary 5.3 and 5.5, the function $\Phi_{k}$ defined by (5.7) satisfies, for $\mu_{\kappa}^{(1)}$-almost all $q \in \mathcal{C}_{\kappa}^{(1)}$, the following identity:

$$
\Phi_{k}\left(q, E_{k}^{+}(q)\right) \equiv \Lambda_{1}(q)+\cdots+\Lambda_{g}(q) .
$$

Let $\left\{c_{1}, \ldots, c_{g}\right\} \subset H^{1}\left(M_{q}, \mathbb{R}\right)$ be any orthonormal system, with respect to the Hodge norm, such that $\left\{c_{1}, \ldots, c_{k}\right\}$ is a basis of $E_{k}^{+}(q)$ and let $c_{i}=\left[\Re\left(m_{i}^{+} q^{1 / 2}\right)\right]$ be the harmonic representation (2.4). By formulas (2) and (3) in Lemma 5.2', (8.27) implies that

$$
B_{q}\left(m_{i}^{+}, m_{j}^{+}\right)=0, \quad k+1 \leq i, j \leq g .
$$

The argument will therefore be concluded by the construction of a subset of the connected component $\mathcal{C}_{\kappa}^{(1)}$ with positive measure (with respect to the measure $\left.\mu_{\kappa}^{(1)}\right)$ where $\left(8.27^{\prime}\right)$ does not hold.

We claim that there exists a compact set of positive measure $\mathcal{P}_{\kappa}^{(1)} \subset \mathcal{C}_{\kappa}^{(1)}$ with the following properties:

(1) All $q \in \mathcal{P}_{\kappa}^{(1)}$ are Oseledec regular points, hence the unstable subspace $E_{k}^{+}(q) \subset H^{1}(M, \mathbb{R})$ is defined and it is a $k$-dimensional (isotropic) subspace. The mapping $q \rightarrow E_{k}^{+}(q) \subset H^{1}(M, \mathbb{R})$ is continuous on $\mathcal{P}_{\kappa}^{(1)}$.

(2) For any $\varepsilon>0$, the flat structure induced by $q \in \mathcal{P}_{\kappa}^{(1)}$, that is the flat Riemannian surface with singularities $\left(M, R_{q}\right)$, contains $g \geq 2$ embedded metric cylinders $A_{1}^{\varepsilon}, \ldots, A_{g}^{\varepsilon}$ (in the sense of the Definition in [45, p. 457]) with non-horizontal waist curves $\gamma_{1}^{\varepsilon}, \ldots, \gamma_{g}^{\varepsilon}$ such that $M \backslash \cup\left\{\gamma_{1}^{\varepsilon}, \ldots, \gamma_{g}^{\varepsilon}\right\}$ is homeomorphic to a sphere minus $2 g$ disjoint disks and

$$
d_{q}\left(E_{k}^{+}(q), P\left(\Gamma^{\varepsilon}\right)\right)<\varepsilon,
$$

where $P\left(\Gamma^{\varepsilon}\right) \subset H^{1}(M, \mathbb{R})$ is the Poincaré dual of the (Lagrangian) subspace $\Gamma^{\varepsilon} \subset H_{1}(M, \mathbb{R})$ generated by the homology classes of the waist curves $\gamma_{1}^{\varepsilon}, \ldots, \gamma_{g}^{\varepsilon}$ and $d_{q}$ denotes the metric induced on the Grassmannian by the Hodge inner product on $H^{1}\left(M_{q}, \mathbb{R}\right)$. 
Let $q_{0} \in \mathcal{C}_{\kappa}^{(1)}$ a quadratic differential with the properties listed in the statement of Lemma 8.4. The existence of such a differential in every connected component of $\mathcal{M}_{\kappa}^{(1)}$ follows immediately from the density property asserted by the lemma. Since the horizontal foliation $\mathcal{F}_{q_{0}}$ is Lagrangian, the flat structure induced by $q_{0}$ contains at least $g \geq 2$ flat horizontal cylinders with waist curves $\gamma_{1}\left(q_{0}\right), \ldots, \gamma_{g}\left(q_{0}\right)$ which generate a Lagrangian subspace $\mathcal{L}_{0} \subset H_{1}\left(M_{q_{0}}, \mathbb{R}\right)$. There exists a neighbourhood $\mathcal{U}_{\kappa}^{(1)} \subset \mathcal{C}_{\kappa}^{(1)}$ of $q_{0}$ such that any $q \in \mathcal{U}_{\kappa}^{(1)}$ has $g \geq 2$ distinct closed (possibly non-horizontal) regular trajectories $\gamma_{1}(q), \ldots, \gamma_{g}(q)$, isotopic respectively to $\gamma_{1}\left(q_{0}\right), \ldots, \gamma_{g}\left(q_{0}\right)$. Let $\mathcal{P}_{\kappa}^{(1)} \subset \mathcal{U}_{\kappa}^{(1)}$ be a compact set of positive measure with the properties listed in Lemma 8.4.

Let $q \in \mathcal{P}_{\kappa}^{(1)}$ and let $T<0$ be a backward return time of the Teichmüller orbit $G_{t}(q)$ to the compact set of positive measure $\mathcal{P}_{\kappa}^{(1)}$. The quadratic differential $q_{T}:=G_{T}(q)$ has therefore $g \geq 2$ closed non-horizontal regular trajectories $\gamma_{1}\left(q_{T}\right), \ldots, \gamma_{g}\left(q_{T}\right)$ which generate in the homology vector space the Lagrangian plane $\mathcal{L}_{0}$ with Poincaré dual $P\left(\mathcal{L}_{0}\right)$ transverse to $E^{-}\left(q_{T}\right)$. Let $\Gamma^{T} \subset H_{1}\left(M_{q}, \mathbb{Z}\right)$ be the Lagrangian subspace generated by the homology classes of the closed non-horizontal regular $q$-trajectories $\gamma_{1}^{T}(q), \ldots, \gamma_{g}^{T}(q)$, obtained from $\gamma_{1}\left(q_{T}\right), \ldots, \gamma_{g}\left(q_{T}\right)$ by pull-back under the Teichmüller map $f_{T}:(M, q) \rightarrow\left(M, q_{T}\right)$. Let $P\left(\Gamma_{T}\right) \subset H^{1}\left(M_{q}, \mathbb{R}\right)$ be the Poincaré dual of the subspace $\Gamma_{T}$. By definition of the Kontsevich-Zorich cocycle, $P\left(\Gamma_{T}\right)=$ $G_{-T}^{K Z}\left(P\left(\mathcal{L}_{0}\right)\right)$. Since $P\left(\mathcal{L}_{0}\right)$ is transverse to $E^{-}\left(q_{T}\right)$, by the Oseledec's theorem, for any $\varepsilon>0$ there exists $T_{\varepsilon}<0$ such that, if the return time $T<T_{\varepsilon}$, then the Lagrangian subspace $P\left(\Gamma^{\varepsilon}\right):=P\left(\Gamma_{T}\right)$ satisfies the estimate (8.28). We therefore choose a return time $T<T_{\varepsilon}$ and let $\left\{\gamma_{1}^{\varepsilon}, \ldots, \gamma_{g}^{\varepsilon}\right\}:=\left\{\gamma_{1}^{T}(q), \ldots, \gamma_{g}^{T}(q)\right\}$. The embedded metric cylinders $A_{1}^{\varepsilon}, \ldots, A_{g}^{\varepsilon}$ are then determined by the closed regular non-horizontal $q$-trajectories $\gamma_{1}^{\varepsilon}, \ldots, \gamma_{g}^{\varepsilon}$. We have thus constructed a compact set of positive measure $\mathcal{P}_{\kappa}^{(1)} \subset \mathcal{C}_{\kappa}^{(1)}$ with the required properties.

Let $\left\{\gamma_{1}, \ldots, \gamma_{g}\right\}$ be a set of disjoint simple closed curves on $M$ such that $M \backslash\left\{\gamma_{1}, \ldots, \gamma_{g}\right\}$ is homeomorphic to a sphere minus $2 g$ disjoint (paired) disks. Let $\mathcal{U}_{\kappa}\left(\gamma_{1}, \ldots, \gamma_{g}\right)$ be the open set of quadratic differentials $q \in Q_{\kappa}$ having closed regular non-horizontal trajectories $\gamma_{1}(q), \ldots, \gamma_{g}(q)$ isotopic respectively to $\gamma_{1}, \ldots, \gamma_{g}$. We will construct a smooth deformation

$$
\Psi:(0,1]^{g} \times \mathcal{U}_{\kappa}\left(\gamma_{1}, \ldots, \gamma_{g}\right) \rightarrow \mathcal{U}_{\kappa}\left(\gamma_{1}, \ldots, \gamma_{g}\right)
$$

having the properties listed below. Let $q_{t}:=\Psi_{t}(q), t=\left(t_{1}, \ldots, t_{g}\right) \in(0,1]^{g}$, then

(1) if $t_{i}=1$ for all $i=1, \ldots, g$, then $q_{t}=q$.

(2) if $t_{i} \rightarrow 0$ for all $i=1, \ldots, g$, then the Riemann surface carrying $q_{t}$ converges to a Riemann surface with nodes, pinched along the curves $\gamma_{1}, \ldots, \gamma_{g}$, hence $q_{t}$ converges in the moduli space $\mathcal{M}_{g}$ to a quadratic 
differential $q_{0} \in \mathcal{S}_{g}$, the boundary component of the moduli space $\mathcal{M}_{g}$ consisting of regular quadratic differentials on the Riemann sphere with $2 g$ paired punctures, having poles of order 2 at all punctures (considered in $\S 4)$.

(3) the zero set of $q_{t}$ coincides with the zero set $\Sigma_{q}$ of $q$ for all $t \in(0,1]^{g}$ and the cohomology class $\left[\Im\left(q_{t}^{1 / 2}\right)\right]=\left[\Im\left(q^{1 / 2}\right)\right] \in H^{1}\left(M, \Sigma_{q}, \mathbb{R}\right)$.

We will construct $\Psi_{t}(q)$ as a composition of deformations $\Psi_{t}^{(i)}, i=$ $1, \ldots, g$, pinching along $\gamma_{i}$. Let $q \in \mathcal{U}_{\kappa}\left(\gamma_{1}, \ldots, \gamma_{g}\right)$ and let $\theta_{i} \in(0, \pi)$ be the angle between the oriented horizontal foliation $\mathcal{F}_{q}$ and the closed regular oriented trajectory $\gamma_{i}(q)$, isotopic to $\gamma_{i}$. Let $H_{s}^{+}, s \in \mathbb{R}$, be the horocycle flow on $Q_{g}$, given by the action of the one-parameter subgroup of $\mathrm{SL}(2, \mathbb{R})$

$$
H_{s}^{+}:=\left[\begin{array}{ll}
1 & s \\
0 & 1
\end{array}\right]
$$

Let $s_{i}:=\cot \theta_{i} \in \mathbb{R}$. Then the horizontal foliation of the quadratic differential $H_{-s_{i}}^{+}(q) \in Q_{\kappa}$ coincides with $\mathcal{F}_{q}$ and $\gamma_{i}(q)$ is a closed regular vertical trajectory of $H_{-s_{i}}^{+}(q)$. In addition, since the set of closed regular trajectories is invariant under the horocycle action, $H_{-s_{i}}^{+}(q) \in \mathcal{U}_{\kappa}\left(\gamma_{1}, \ldots, \gamma_{g}\right)$. Let $\mathcal{V}_{\kappa}\left(\gamma_{i}\right) \subset \mathcal{U}_{\kappa}\left(\gamma_{1}, \ldots, \gamma_{g}\right)$ be the set of quadratic differentials having a closed regular vertical trajectory $\gamma_{i}(q)$ isotopic to $\gamma_{i}$ defined in the proof of Lemma $4.4^{\prime}$ and let $\Phi^{(i)}:(0,1] \times \mathcal{V}_{\kappa}\left(\gamma_{i}\right) \rightarrow \mathcal{V}_{\kappa}\left(\gamma_{i}\right)$ be the deformation defined by the identity (a) in (4.31). We then define:

(a) $\Psi_{t_{i}}^{(i)}(q):=H_{s_{i}}^{+} \circ \Phi_{t_{i}}^{(i)} \circ H_{-s_{i}}^{+}(q), \quad\left(t_{i}, q\right) \in(0,1] \times \mathcal{U}_{\kappa}\left(\gamma_{1}, \ldots, \gamma_{g}\right)$,

(b) $\Psi_{t}(q):=\Psi_{t_{1}}^{(1)} \circ \cdots \circ \Psi_{t_{g}}^{(g)}(q), \quad(t, q) \in(0,1]^{g} \times \mathcal{U}_{\kappa}\left(\gamma_{1}, \ldots, \gamma_{g}\right)$.

It can be checked that the pinching deformation $\Psi:(0,1]^{g} \times \mathcal{U}_{\kappa}\left(\gamma_{1}, \ldots, \gamma_{g}\right) \rightarrow$ $\mathcal{U}_{\kappa}\left(\gamma_{1}, \ldots, \gamma_{g}\right)$ is well-defined and has the required properties. In fact, by construction, if $q \in \mathcal{U}_{\kappa}\left(\gamma_{1}, \ldots, \gamma_{g}\right)$ and $\theta_{j} \in(0, \pi)$ is the oriented angle of the oriented horizontal foliation $\mathcal{F}_{q}$ and the closed regular non-horizontal oriented $q$-trajectory $\gamma_{j}(q)$, then $\theta_{j}$ is preserved by the deformation $\Psi_{t_{i}}^{(i)}(q), i, j=$ $1, \ldots, g$. Hence, the composition in $(b)$ is well-defined. The properties (1) and (2) hold by the definition (8.31) and by the corresponding properties of the deformations $\Phi_{t_{i}}^{(i)}$. By its construction, in the proof of Lemma $4.4^{\prime}, \Phi_{t_{i}}^{(i)}(q)=q$, if $t_{i}=1$, and $\Phi_{t_{i}}^{(i)}(q)$ converges in the compactified moduli space as $t_{i} \rightarrow 0$, to a regular quadratic differential with 2 paired poles of order 2 and real strictly positive residues on a Riemann surface of genus $g-1$ with 2 punctures. Hence $q_{t}:=\Psi_{t}(q)$ converges, as $t_{i} \rightarrow 0$ for all $i=1, \ldots, g$, to a regular quadratic differential $q_{0} \in \mathcal{S}_{g}$ (on a punctured Riemann sphere with $2 g$ paired punctures) 
and real strictly positive residues at all punctures. Finally, (3) follows from the corresponding property of $\Phi_{t_{i}}^{(i)}$, established in the proof of Lemma $4.4^{\prime}$, since the horocycle flow $\mathrm{H}_{s}^{+}$preserves the horizontal measured foliation.

Let $\left\{a_{1}, \ldots, a_{g}, b_{1}, \ldots, b_{g}\right\}$ be a canonical homology basis on a Riemann surface $M$ and $\left\{\theta_{1}, \ldots, \theta_{g}\right\}$ be the dual basis of holomorphic differentials, defined by the standard condition $\theta_{i}\left(a_{j}\right)=\delta_{i j}$. Let $\mathcal{A} \subset H_{1}(M, \mathbb{R})$ be the Lagrangian subspace generated by the homology classes of the curves $\left\{a_{1}, \ldots, a_{g}\right\}$. By the definition of the Poincaré duality, the Poincaré dual $P(\mathcal{A}) \subset H^{1}(M, \mathbb{R})$ is generated by the cohomology classes of the harmonic forms $\left\{\Im\left(\theta_{1}\right), \ldots, \Im\left(\theta_{g}\right)\right\}$. Let $q \in Q_{\kappa}$ and let $\phi_{i}^{+}:=\theta_{i} / q^{1 / 2} \in L_{q}^{2}(M)$ be the corresponding meromorphic functions. Since $\Im\left(\theta_{i}\right)=-\Re\left(\imath \theta_{i}\right)$, the Lagrangian subspace $P(\mathcal{A})$ is represented, under the $\mathbb{R}$-linear isomorphism defined in (2.4), by the $g$-dimensional real subspace $V_{q}^{+}(\mathcal{A}) \subset \mathcal{M}_{q}^{+}$generated by the system $\left\{\imath \phi_{1}^{+}, \ldots, \imath \phi_{g}^{+}\right\}$. Let $\left\{m_{1}^{+}, \ldots, m_{g}^{+}\right\}$be any orthonormal basis of $V_{q}^{+}(\mathcal{A})$ over $\mathbb{R}$, with respect to the (symmetric) inner product induced on $V_{q}^{+}(\mathcal{A})$ by the hermitian structure of $L_{q}^{2}(M)$. Let $B$ be the symmetric complex matrix defined by $B_{i j}:=B_{q}\left(m_{i}^{+}, m_{j}^{+}\right)$. The matrix $B$ depends on the quadratic differential and on the choice of the orthonormal basis $\left\{m_{1}^{+}, \ldots, m_{g}^{+}\right\}$of $V_{q}^{+}(\mathcal{A})$. By $(4.4)$ and (4.5), we have:

$$
B=C^{-1} \frac{d \Pi}{d \mu_{q}}\left(C^{t}\right)^{-1},
$$

where $C$ is the matrix of base change defined in (4.5) and $\Pi$ is the period matrix with respect to the canonical basis $\left\{a_{1}, \ldots, a_{g}, b_{1}, \ldots, b_{g}\right\}$. Since in the present case the matrix $C$ is purely imaginary, by (4.5) we have that $C C^{t}=-C C^{*}=-\Im(\Pi)$. Let $q_{0} \in \mathcal{S}_{g}$ be a regular quadratic differential on the $2 g$-punctured Riemann sphere with positive residues at all punctures. We claim that, if $q \rightarrow q_{0}$ as $M_{q}$ is pinched along the homotopically non-trivial curves $a_{1}, \ldots, a_{g}$, then

$$
\lim _{q \rightarrow q_{0}} B_{q}\left(m_{i}^{+}, m_{j}^{+}\right)=\delta_{i j},
$$

uniformly with respect to the orthonormal basis $\left\{m_{1}^{+}, \ldots, m_{g}^{+}\right\}$of $V_{q}^{+}(\mathcal{A})$. In fact, by $\left(4.5^{\prime}\right)$ and (4.33),

$$
\left(\phi_{i}^{+}, \phi_{j}^{+}\right)_{q}+\frac{1}{2 \pi} \delta_{i j} \log \left|t_{i}\right|
$$

is bounded as $t \rightarrow 0$, where $t=\left(t_{1}, \ldots, t_{g}\right)$ are the pinching parameters, described in $\S 4$, corresponding respectively to the curves $a_{1}, \ldots, a_{g}$. It follows that the purely imaginary matrix $C$ of base change converges, up to left multiplication by a diagonal purely imaginary matrix, to the compact subgroup $\mathrm{O}(g, \mathbb{R}) \subset \mathrm{GL}(g, \mathbb{R})$ of real $g \times g$ orthogonal matrices. In fact, let $\Delta$ be the 
$g \times g$ diagonal matrix with entries

$$
\Delta_{i j}:=i\left|\frac{\log \left|t_{i}\right|}{2 \pi}\right|^{1 / 2} \delta_{i j} .
$$

By the boundedness of (8.34), as $t \rightarrow 0$,

$$
\Delta^{-1} C \rightarrow O(g, \mathbb{R})
$$

On the other hand, by Lemma 4.2 and Lemma $4.2^{\prime}$, as $t \rightarrow 0$,

$$
\Delta^{-1} \frac{d \Pi}{d \mu_{q}} \Delta^{-1} \rightarrow I_{g \times g} .
$$

The claim (8.33) follows then from (8.32), (8.35) and (8.35').

Let $q^{(0)} \in \mathcal{C}_{\kappa}^{(1)}$ be a density point of the compact set of positive measure $\mathcal{P}_{\kappa}^{(1)}$ constructed above. Let $\varepsilon>0$ and let $\left\{\gamma_{1}^{\varepsilon}, \ldots, \gamma_{g}^{\varepsilon}\right\}$ be the system of disjoint closed curves with the properties stated in the description of $\mathcal{P}_{\kappa}^{(1)}$ at (2) (see p. 75). Let $q_{t}^{(0)}=\Psi_{t}\left(q^{(0)}\right)$ be the pinching deformation along the curves $\gamma_{1}^{\varepsilon}, \ldots, \gamma_{g}^{\varepsilon}$ constructed above. Since $q_{t}^{(0)}$ converges as $t \rightarrow 0$ to a regular quadratic differential $q_{0}^{(0)} \in \mathcal{S}_{g}$ with real strictly positive residues and there exists a canonical homology basis $\left\{a_{1}, \ldots, a_{g}, b_{1}, \ldots, b_{g}\right\}$ such that $a_{i}=\gamma_{i}^{\varepsilon}$, for all $i \in\{1, \ldots, g\}$, by (8.33) there exists $\tau>0$ such that the following holds. Let $t \in(0,1]^{g}$ with $|t|<\tau$ and $q:=q_{t}^{(0)}$. Let $\left\{c_{1}, \ldots, c_{g}\right\} \subset H^{1}\left(M_{q}, \mathbb{R}\right)$ be any orthonormal basis, with respect to the Hodge norm, of the Poincaré dual $P\left(\Gamma^{\varepsilon}\right)$ of the Lagrangian subspace $\Gamma^{\varepsilon} \subset H_{1}\left(M_{q}, \mathbb{R}\right)$ generated by the homology classes of the curves $\gamma_{1}^{\varepsilon}, \ldots, \gamma_{g}^{\varepsilon}$ and let $c_{i}=\left[\Re\left(m_{i}^{+} q^{1 / 2}\right)\right] \in H^{1}\left(M_{q}, \mathbb{R}\right)$ be the harmonic representation (2.4). Then

$$
\left|B_{q}\left(m_{i}^{+}, m_{i}^{+}\right)\right|>\frac{1}{2}, \quad i=1, \ldots, g .
$$

Let us choose $t^{*} \in(0,1]^{g}$ such that $\left|t^{*}\right|<\tau$ and let $\Psi_{*}:=\Psi_{t}$ for $t=t^{*}$. There exists an open neighbourhood $\mathcal{U}_{\kappa}^{(0)} \subset \mathcal{U}_{\kappa}\left(a_{1}, \ldots, a_{g}\right)$ such that $q^{(0)} \in \mathcal{U}_{\kappa}^{(0)}$, (8.28) holds for all $q \in \mathcal{U}_{\kappa}^{(0)} \cap \mathcal{P}_{\kappa}^{(1)}$ and (8.36) holds for all $q \in \Psi_{*}\left(\mathcal{U}_{\kappa}^{(0)}\right)$. In fact, the first assertion follows from the fact that, by construction, $E_{k}^{+}(q)$ depends continuously on $q \in \mathcal{P}_{\kappa}^{(1)}$, the latter from the continuity of the bilinear form $B_{q}$. Finally, since the inequality (8.28) is preserved at any Oseledec regular point of the form $\Psi_{t}(q), q \in \mathcal{P}_{\kappa}^{(1)}$, by property (3) of $\Psi_{t}$, Lemma 6.8 and Theorem 8.3 , if $\varepsilon>0$ is chosen sufficiently small, the subset of regular points of the set $\Psi_{*}\left(\mathcal{U}_{\kappa}^{(0)} \cap \mathcal{P}_{\kappa}^{(1)}\right)$ yields a subset of $\mathcal{C}_{\kappa}^{(1)}$ of positive measure (with respect to $\mu_{\kappa}^{(1)}$ ) where the identities $\left(8.27^{\prime}\right)$ fail for all $i=j \in\{k+1, \ldots, g\}$.

8.3. The Oseledec's theorem for the bundle of closed currents of order 1 . By Theorem 8.3, which describes the invariant sub-bundles of the KontsevichZorich cocycle in terms of basic currents of order 1, and Theorem 8.5, which 
proves the (non-uniform) hyperbolicity of the cocycle, we can derive an Oseledec's theorem for the restriction of the cocycle $G_{t}^{c}$ to the bundle of all closed currents of order 1. This is a crucial step in order to obtain results on the deviation of ergodic averages for measured foliations. In fact, by the standard trace theorem for Sobolev spaces [1, Th. 5.4 (5)], the (return) leaves of a 1-dimensional foliation on a 2-dimensional manifold can be regarded as currents of order 1 .

LEMma 8.6. The forward and backward Lyapunov spectra of the cocycle $G_{t}^{0}$ on the Hilbert bundle $H_{\kappa}^{-1}(M)$, defined by (8.1), are contained in the closed interval $[-1,1]$, in the sense that, for all $q \in \mathcal{M}_{\kappa}^{(1)}$ and any $\mathcal{D} \in H_{q}^{-1}(M)$,

$$
-1 \leq \liminf _{t \rightarrow \pm \infty} \frac{1}{|t|} \log \left|G_{t}^{0}(\mathcal{D})\right|_{-1} \leq \limsup _{t \rightarrow \pm \infty} \frac{1}{|t|} \log \left|G_{t}^{0}(\mathcal{D})\right|_{-1} \leq 1 .
$$

The forward and backward Lyapunov spectra of the cocycle $G_{t}^{c}$ on the Hilbert bundle $\mathcal{H}_{\kappa}^{-1}(M)$, defined by $(8.2),\left(8.2^{\prime}\right)$, are contained in the closed interval $[-2,2]$, in the sense that, for all $q \in \mathcal{M}_{\kappa}^{(1)}$ and any $C \in \mathcal{H}_{q}^{-1}(M)$,

$$
-2 \leq \liminf _{t \rightarrow \pm \infty} \frac{1}{|t|} \log \left|G_{t}^{c}(C)\right|_{-1} \leq \limsup _{t \rightarrow \pm \infty} \frac{1}{|t|} \log \left|G_{t}^{c}(C)\right|_{-1} \leq 2 .
$$

There is a continuous (orthogonal) $G_{t}^{c}$-invariant splitting

$$
\mathcal{H}_{\kappa}^{-1}(M):=I_{\kappa}^{-}(M) \oplus I_{\kappa}^{+}(M)
$$

into the closed infinite-dimensional sub-bundles $I_{\kappa}^{ \pm}(M)$ with fiber at $q \in \mathcal{M}_{\kappa}^{(1)}$ given by

$$
\begin{aligned}
I_{q}^{+}(M) & :=\left\{C \in \mathcal{H}_{\kappa}^{-1}(M) \mid \imath_{S} C=0\right\}, \\
I_{q}^{-}(M) & :=\left\{C \in \mathcal{H}_{\kappa}^{-1}(M) \mid \imath_{T} C=0\right\} .
\end{aligned}
$$

The restriction of the cocycle $G_{t}^{c}$ to $I_{\kappa}^{+}(M)\left[I_{\kappa}^{-}(M)\right]$ has non-negative [nonpositive] forward Lyapunov spectrum. A corresponding statement, reversing the role of $I_{\kappa}^{+}(M)$ and $I_{\kappa}^{-}(M)$, holds for the backward spectrum.

Proof. Since the $L_{q}^{2}$ norm is invariant under the Teichmüller flow $G_{t}$, by $\left(2.7^{\prime}\right)$ and the definition (8.1) of $G_{t}^{0}$, we have:

$$
e^{-|t|}|\mathcal{D}|_{-1} \leq\left|G_{t}^{0}(\mathcal{D})\right|_{-1} \leq e^{|t|}|\mathcal{D}|_{-1},
$$

where $|\cdot|_{-1}$ denotes the norm induced on the fiber at any $q \in \mathcal{M}_{\kappa}^{(1)}$ by the Hilbert structure of $H_{q}^{-1}(M)$. The bounds (8.37) follow immediately from (8.39). 
The sub-bundles $I_{\kappa}^{ \pm}(M)$ are $G_{t}^{c}$-invariant by definition and, by the definition (8.2) of $G_{t}^{c}$, the Lyapunov spectra of $G_{t}^{c}$ on $I_{\kappa}^{ \pm}(M)$ can be obtained from the Lyapunov spectrum of $G_{t}^{0}$ by translation of \pm 1 respectively. The orthogonal splitting (8.38) holds since $M$ has dimension 2 and the bounds $\left(8.37^{\prime}\right)$ follow from the bounds (8.37) and the splitting (8.38).

THEOREM 8.7. The infinite dimensional closed sub-bundle $\mathcal{Z}_{\kappa}^{1}(M) \subset$ $\mathcal{H}_{\kappa}^{-1}(M)$ of closed currents of order 1 is $G_{t}^{c}$-invariant and has a measurable $G_{t}^{c}$-invariant splitting:

$$
\mathcal{Z}_{\kappa}^{1}(M)=\mathcal{B}_{\kappa,+}^{1}(M) \oplus \mathcal{B}_{\kappa,-}^{1}(M) \oplus \mathcal{E}_{\kappa}^{1}(M) .
$$

(1) The measurable sub-bundles $\mathcal{B}_{\kappa, \pm}^{1}(M)$ have fibers equal to $\mathcal{B}_{ \pm q}^{1}(M)$, the finite dimensional vector space of basic currents of order 1 for $\mathcal{F}_{ \pm q}$, at $\mu_{\kappa}^{(1)}$-almost all quadratic differentials $q \in \mathcal{M}_{\kappa}^{(1)}$. The sub-bundle $\mathcal{E}_{\kappa}^{1}(M) \subset$ $\mathcal{Z}_{\kappa}^{1}(M)$ is the closed infinite dimensional continuous bundle of exact currents, which has everywhere defined fibers $\mathcal{E}_{q}^{1}(M):=\left\{d U \mid U \in L_{q}^{2}(M)\right\}$.

(2) The restriction of the cocycle $G_{t}^{c}$ to the bundle

$$
\mathcal{B}_{\kappa}^{1}(M)=\mathcal{B}_{\kappa,+}^{1}(M) \oplus \mathcal{B}_{\kappa,-}^{1}(M)
$$

is measurably isomorphic to the Kontsevich-Zorich cocycle $G_{t}^{K Z}$ on the real cohomology bundle $\mathcal{H}_{\kappa}^{1}(M, \mathbb{R})$, hence it has the Lyapunov spectrum (8.26). The invariant sub-bundles $\mathcal{B}_{\kappa, \pm}^{1}(M)$ correspond to the (strictly) positive, respectively (strictly) negative, Lyapunov exponents. The Lyapunov spectrum of $G_{t}^{c}$ on $\mathcal{E}_{\kappa}^{1}(M)$ is reduced to the single Lyapunov exponent 0 .

Proof. The bundle $\mathcal{Z}_{\kappa}^{1}(M)$ and the splitting (8.40) are $G_{t}^{c}$-invariant by the definition (8.2), $\left(8.2^{\prime}\right)$ of the cocycle $G_{t}^{c}$. By Lemma 8.1, Theorem 8.3 and Theorem 8.5, the restriction of $G_{t}^{c}$ to the sub-bundle $\mathcal{B}_{\kappa}^{1}(M)$ is measurably isomorphic to the Kontsevich-Zorich cocycle, hence it has Lyapunov spectrum given by (8.26). In fact, the restriction of the cohomology map $j_{\kappa}$, defined by $\left(8.3^{\prime}\right)$, to $\mathcal{B}_{\kappa}^{1}(M)$ is injective by Lemma $8.1(4)$, and surjective by Theorem 8.3 and Theorem 8.5. By Lemma 8.1(1), it follows that $j_{\kappa}$ is an isomorphism of the cocycle $G_{t}^{c}$ onto the Kontsevich-Zorich cocycle $G_{t}^{K Z}$. By Lemma 8.1(3), the Lyapunov exponents of $G_{t}^{c}$ on $\mathcal{B}_{\kappa,+}^{1}(M)$ [on $\mathcal{B}_{\kappa,-}^{1}(M)$ ] are strictly positive [strictly negative].

The forward and backward Lyapunov spectra of the restriction of the cocycle $G_{t}^{c}$ to the sub-bundle $\mathcal{E}_{\kappa}^{1}(M)$ of exact currents are reduced to the Lyapunov exponent 0 . In fact, since $|d U|_{-1} \leq|U|_{0}$ and the $L_{q}^{2}$ norm is invariant under the Teichmüller flow,

$$
\limsup _{t \rightarrow \pm \infty} \frac{1}{|t|} \log \left|G_{t}^{c}(d U)\right|_{-1} \leq \limsup _{t \rightarrow \pm \infty} \frac{1}{|t|} \log |U|_{0}=0 .
$$


On the other hand, if the horizontal and vertical foliations $\mathcal{F}_{ \pm q}$ are ergodic, $d U \neq 0$ implies that the currents $(\mathrm{SU})^{*} \eta_{T} \in I_{q}^{-}(M)$ and $(T U)^{*} \eta_{S} \in I_{q}^{+}(M)$, which are the projections of $d U$ given by the splitting (8.38) are non-zero. Hence, by Lemma 8.6,

$$
\begin{aligned}
& \liminf _{t \rightarrow+\infty} \frac{1}{t} \log \left|G_{t}^{c}(d U)\right|_{-1} \geq \liminf _{t \rightarrow+\infty} \frac{1}{t}\left|G_{t}^{c}\left((T U)^{*} \eta_{S}\right)\right|_{-1} \geq 0, \\
& \liminf _{t \rightarrow-\infty} \frac{1}{|t|} \log \left|G_{t}^{c}(d U)\right|_{-1} \geq \liminf _{t \rightarrow-\infty} \frac{1}{|t|}\left|G_{t}^{c}\left((\mathrm{SU})^{*} \eta_{T}\right)\right|_{-1} \geq 0 .
\end{aligned}
$$

Remark 8.7'. There is no exhaustive theory of Lyapunov exponents for cocycles on Hilbert or Banach bundles. In particular, the available general results ([55] and references therein) do not seem to apply to the cocycles $G_{t}^{0}$ and $G_{t}^{c}$. It is possible to deduce from Theorem 8.7 some additional information concerning the Lyapunov spectrum of $G_{t}^{0}$, namely that its forward Lyapunov spectrum contains the Lyapunov exponents

$$
-1+\lambda_{g}<\cdots<-1+\lambda_{2}<0<1-\lambda_{2}<\cdots<1-\lambda_{g}
$$

but no Oseledec's decomposition of the bundle $H_{\kappa}^{-1}(M)$ seems to exist. The Lyapunov spectrum of $G_{t}^{c}$ can be obtained from that of $G_{t}^{0}$ as the union of the translate by +1 and of the translate by -1 .

\section{The deviation of ergodic averages and open questions}

In this conclusive section of the paper we apply the results we have obtained on the Kontsevich-Zorich cocycle and on the cocycle $G_{t}^{c}$ on the bundle of closed currents of order 1 to prove results concerning the deviation of the ergodic averages of measured foliations and of area-preserving smooth vector fields on higher genus surfaces. By Theorem 8.7 and by Sobolev estimates on the first return orbits as 1-dimensional currents of order 1, we derive Sobolev estimates valid for a sequence of 'best' return times. Then by a standard technical argument we deduce estimates for all times.

9.1. The $L^{2}$ mean deviation and the cohomological equation. By the Gottschalk-Hedlund theorem, there is a relation between the deviation of ergodic averages and the existence of solutions of the cohomological equation. The topological version of the theorem, which states that the cohomological equation $X u=f$, for a minimal flow generated by a vector field $X$, has a continuous solution $u$ if and only if the ergodic integrals of $f$ are uniformly bounded, was first proved in [22, Th. 14.11]. A proof can also be found in [32, Th. 2.9.4]. A similar theorem can be proved for $L^{2}$ solutions, although we were not able to find a reference. By the $L^{2}$ version of the Gottschalk-Hedlund 
theorem, Theorem 8.3 yields a negative answer to a question, left open in [18], concerning the existence of $L^{2}$ solutions of the cohomological equation. We therefore prove that the statement of $[17, \mathrm{Th} . \mathrm{A}]$ is incorrect and should be replaced by $[18$, Th. A].

THEOREM 9.1. For $\mu_{\kappa}^{(1)}$-almost all quadratic differentials $q \in \mathcal{M}_{\kappa}^{(1)}$ there exists (at least) a function $f \in C_{0}^{\infty}\left(M \backslash \Sigma_{q}\right)$, with zero average, such that the cohomological equation $S u=f$ has no solution $u \in L_{q}^{2}(M)$. Let $\Phi_{q}^{\mathcal{T}}$ be the (almost everywhere defined) flow of the vector field $S$, by the GottschalkHedlund theorem,

$$
\limsup _{\mathcal{T} \rightarrow+\infty}\left|\int_{0}^{\mathcal{T}} f\left(\Phi_{q}(p, \tau)\right) d \tau\right|_{0}=+\infty
$$

Proof. Assume that the cohomological equation $S u=f$ has a solution $u \in L_{q}^{2}(M)$ for any $f \in C_{0}^{\infty}\left(M \backslash \Sigma_{q}\right)$. Then by the methods of Section 7.1 it would be possible to construct a $2 g-1$ dimensional subspace of basic currents of order 1 for the horizontal foliation $\mathcal{F}_{q}$. In fact, given any meromorphic function $m^{+} \in \mathcal{M}_{q}^{+}$with zero average, the cohomological equation $S u=-\Re\left(m^{+}\right)$ would have a solution $u \in L_{q}^{2}(M)$. The solution could be constructed as follows. Let $u_{0}$ be a smooth local solution of the equation $S u_{0}=-\Re\left(m^{+}\right)$in a neighbourhood of the zero set $\Sigma_{q}$, which can be obtained by Laurent expansion at the points of $\Sigma_{q}$ and by the formulas (7.4). Let then $u_{1} \in L_{q}^{2}(M)$ be a solution of the equation $S u_{1}=f_{1}$, where $f_{1}:=-\Re\left(m^{+}\right)-S u_{0} \in C_{0}^{\infty}\left(M \backslash \Sigma_{q}\right)$. The function $u:=u_{0}+u_{1} \in L_{q}^{2}(M)$ is therefore a solution of the cohomological equation $S u=-\Re\left(m^{+}\right)$. By formula (7.3), we could then construct a space of basic currents of order 1 for $\mathcal{F}_{q}$ of dimension $2 g-1$. In fact, we would obtain that the basic cohomology $H_{q}^{1,1}(M, \mathbb{R})$ has codimension 1 in $H^{1}(M, \mathbb{R})$, hence dimension $2 g-1$. On the other hand, by Theorem 8.3, for almost all $q \in \mathcal{M}_{\kappa}^{(1)}, H_{q}^{1,1}(M, \mathbb{R})$ coincides with the unstable subspace of the KontsevichZorich cocycle, hence it has dimension (at most) equal to the genus $g \geq 2$. Since $2 g-1>g \geq 2$, we have obtained a contradiction. Hence, we have proved that there exists $f \in C_{0}^{\infty}\left(M \backslash \Sigma_{q}\right)$ with zero average such that the cohomological equation $S u=f$ has no solution $u \in L_{q}^{2}(M)$.

By the Gottschalk-Hedlund theorem, if (9.1) were false, the cohomological equation $S u=f$ would have a solution $u \in L_{q}^{2}(M)$. In fact, in that case the one-parameter family

$$
u_{\mathcal{T}}(p):=-\frac{1}{\mathcal{T}} \int_{0}^{\mathcal{T}} \int_{0}^{\tau} f\left(\Phi_{q}(p, s)\right) d s d \tau
$$


would be bounded in $L_{q}^{2}(M)$. In addition, a computation shows that, if $\mathcal{F}_{q}$ is ergodic,

$$
S u_{\mathcal{T}}(p)=f(p)-\frac{1}{\mathcal{T}} \int_{0}^{\mathcal{T}} f\left(\Phi_{q}(p, \tau)\right) d \tau \rightarrow f \text { in } L_{q}^{2}(M)
$$

Any weak limit $u \in L_{q}^{2}(M)$ of the family (9.2) would therefore be a solution of the equation $S u=f$. Since we proved above that such an equation has no solution $u \in L_{q}^{2}(M)$, it follows that (9.1) holds.

The non-existence of $L_{q}^{2}(M)$ solutions for a cohomological equation $S u=f$, with $f$ smooth and of zero average is therefore related to the existence of unbounded deviation in the ergodic integrals of $f$. In the case of the torus $T^{2}(g=1)$ this phenomenon does not occur for a full measure set of flows (given by a Diophantine condition). In the case of higher genus, as it was conjectured in [38], the deviation of the ergodic integrals from the linear growth behaviour predicted by the ergodic theorem obeys, for a typical function, a power law with exponent $\lambda_{2}>0$.

9.2. Sobolev estimates for the (first) return orbits. The key idea of our argument consists in studying the dynamics of the flow $G_{t}^{c}$ on the infinitedimensional non-closed sub-bundles $\Gamma_{\kappa}^{ \pm} \subset \mathcal{H}_{\kappa}^{-1}(M)$ generated by segments of leaves of the horizontal and vertical foliations. Let $\mathcal{T}>0$. We denote by $\gamma_{ \pm q}^{\mathcal{T}}$ a positively oriented segment of length $\mathcal{T}>0$ of a leaf of the measured foliation $\mathcal{F}_{ \pm q}$ respectively. By trace theorems for Sobolev spaces, the vector spaces $\Gamma_{q}^{ \pm}$generated by the segments $\gamma_{ \pm q}^{\mathcal{T}}$ are subspaces of the space $\mathcal{H}_{q}^{-1}(M)$ of 1-dimensional currents of order 1 . In addition, they are invariant under the action of the cocycle $G_{t}^{c}$. Let $\Phi_{ \pm q}^{\mathcal{T}}, \mathcal{T} \in \mathbb{R}$, be the (almost everywhere defined) flows of the vector fields $S, T$ respectively. The foliations $\mathcal{F}_{ \pm q}$ are almost everywhere the orbit foliations of the flows $\Phi_{ \pm q}^{\mathcal{T}}$. Let $\alpha:=f^{+} \eta_{T}+f^{-} \eta_{S} \in$ $\mathcal{H}_{q}^{1}(M)$, then

$$
\gamma_{ \pm q}^{\mathcal{T}}(\alpha)=\int_{0}^{\mathcal{T}} f^{ \pm}\left(\Phi_{ \pm q}\left(p^{ \pm}, \tau\right)\right) d \tau
$$

where $p^{ \pm} \in M$ are the starting points of the oriented segments $\gamma_{ \pm q}^{\mathcal{T}}$. The ergodic averages of the functions $f^{ \pm} \in H_{q}^{1}(M)$ can therefore be understood by studying the dynamics of the 'renormalization' cocycle $G_{t}^{c}$ on $\Gamma_{\kappa}^{ \pm}$.

The first step consists in an estimate of the norm of $\gamma_{ \pm q}^{\mathcal{T}}$ in $\mathcal{H}_{q}^{-1}(M)$. Let $\|q\|$ denote the $R_{q}$-length of the shortest saddle connection of the quadratic differential $q \in \mathcal{M}_{\kappa}^{(1)}$. 
Lemma 9.2. There exists a constant $K>0$ such that, for all quadratic differentials $q \in \mathcal{M}_{\kappa}^{(1)}$,

$$
\left|\gamma_{ \pm q}^{\mathcal{T}}\right|_{\mathcal{H}_{q}^{-1}(M)} \leq K\left(1+\frac{\mathcal{T}}{\|q\|}\right)
$$

Proof. Let $d_{q}(M)$ be the maximum distance of any point in $M$ to a zero. By [45, Cor. 5.6] there is a constant $K_{1}>1$ such that $d_{q}(M) \leq K_{1} /\|q\|$ or $d_{q}(M) \leq \sqrt{2 / \pi}$. Since $\|q\| / 2 \leq d_{q}(M)$, it follows that there is $K_{2}>1$ such that $\|q\| \leq K_{2}$. Let $\delta:=\|q\| / 3$. Let $\gamma_{+}:=\gamma_{+q}^{\mathcal{T}}$ be a regular segment of length $\mathcal{T}>0$ of a leaf of the horizontal foliation $\mathcal{F}_{q}$. The case of a regular segment $\gamma_{-}:=\gamma_{-q}^{\mathcal{T}}$ of a leaf of the vertical foliation is similar. For all but a finite number of points $p \in \gamma_{+}$, the vertical segment $\gamma_{-}^{\delta}(p)$ of length $2 \delta$, centered at $p$, is well-defined. At the exceptional points $p_{1}, \ldots, p_{N} \in \gamma_{+}$(ordered in the positive direction along $\gamma_{+}$) the vertical leaf through $p_{i}$ meets a singularity at a distance from $p_{i}$ less than $\delta$. Let $\gamma_{+}\left(p_{i}, p_{i+1}\right)$ be the open horizontal segment with endpoints $p_{i}$, $p_{i+1}$. By the definition of $\delta>0$, the $R_{q}$-length $L_{q}\left(\gamma_{+}\left(p_{i}, p_{i+1}\right)\right) \geq \delta$. Otherwise, there would be two singularities at a distance strictly less than $3 \delta=\|q\|$, in contradiction with the definition of $\|q\|$. By possibly introducing additional points, we can assume that the following holds:

$$
\begin{array}{r}
\delta \leq L_{q}\left(\gamma_{+}\left(p_{i}, p_{i+1}\right)\right) \leq 2 \delta, \quad 1 \leq i \leq N-1, \\
L_{q}\left(\gamma_{+}\left(p_{0}, p_{1}\right)\right), L_{q}\left(\gamma_{+}\left(p_{N}, p_{N+1}\right)\right) \leq 2 \delta,
\end{array}
$$

where $p_{0}, p_{N+1}$ are the endpoints (ordered in the positive direction) of $\gamma_{+}$. Let $R_{i} \subset \mathbb{R}^{2}, 0 \leq i \leq N$, be the open rectangles defined by

$$
R_{i}:=\left\{(x, y) \in \mathbb{R}^{2} \mid 0<x<L_{q}\left(\gamma_{+}\left(p_{i}, p_{i+1}\right)\right),-\delta \leq y \leq \delta\right\} .
$$

We claim that there are isometric embeddings of the rectangles $R_{i}$ into $\left(M, R_{q}\right)$ such that the image of the horizontal segments $\gamma_{i}=R_{i} \cap\{y=0\}$ coincide with $\gamma_{+}\left(p_{i}, p_{i+1}\right)$. In fact, let $p \neq p^{\prime} \in \gamma_{+}\left(p_{i}, p_{i+1}\right)$. If $\gamma_{-}^{\delta}(p) \cap \gamma_{-}^{\delta}\left(p^{\prime}\right) \neq \emptyset$, then, by the Pythagorean theorem, there would be a homotopically non-trivial regular closed geodesic of length at most $\sqrt{4 \delta^{2}+4 \delta^{2}}<3 \delta=\|q\|$. Hence, there would be an embedded cylinder with waist of length $<\|q\|$ [58, Chap. IV, §9.3]. The boundary of such a cylinder should then contain at least one saddle connection of smaller or equal length, in contradiction with the definition of $\|q\|$.

Let $R_{a, b}:=\left\{(x, y) \in \mathbb{R}^{2} \mid 0<x<a,-b<y<b\right.$. By a rescaling argument, that is, by reducing to the case of $a=b=1$ by an affine change of coordinate and by the Sobolev trace theorem [1, Th. 5.4 (5)], there exists a constant $K_{3}>0$, such that

$$
\left|\int_{0}^{a} f(x, 0) d x\right| \leq K_{3}\left(\frac{a}{b}\right)^{1 / 2} \max \{a, b, 1\}|f|_{H^{1}\left(R_{a, b}\right)} .
$$


By applying the inequality (9.7) to each of the rectangles $R_{i} \subset M_{q}, 0 \leq i \leq N$, by (9.3) we get

$$
\left|\gamma_{i}(\alpha)\right|=\left|\int_{\gamma_{i}} f^{+} \eta_{T}\right| \leq K_{4}\left|f^{+}\right|_{1} \leq K_{4}|\alpha|_{1},
$$

where $K_{4}:=2^{1 / 2} K_{2} K_{3}$. The estimate (9.4) is then a consequence of $\left(9.7^{\prime}\right)$ and of the inequality $N-1 \leq \mathcal{T} / \delta$, which follows from the first inequality of (9.5).

A point $p \in M$ is regular with respect to a measured foliation $\mathcal{F}$ if it does not belong to a singular leaf of $\mathcal{F}$. Let $q \in \mathcal{M}_{\kappa}^{(1)}$. A point $p \in M$ will be said to be $q$-regular if it is regular with respect to the horizontal and vertical foliations $\mathcal{F}_{ \pm q}$. The set of $q$-regular points is of full measure and it is equivariant under the action of the mapping class group and of the Teichmüller flow. Let $p \in M$ be $q$-regular and let $I_{ \pm q}(p)$ be the vertical [horizontal] segment of length $\|q\| / 2$ centered at $p$. A forward horizontal [vertical] return time of the point $p \in M$ is defined to be any real number $\mathcal{T}_{ \pm q}(p)>0$ such that $\Phi_{ \pm q}\left(p, \mathcal{T}_{ \pm q}(p)\right) \in I_{\mp q}(p)$. If $\mathcal{T}>0$ is any horizontal [vertical] return time of a $q$-regular point $p \in M$, the horizontal [vertical] forward segment $\gamma_{ \pm q}^{\mathcal{T}}(p)$, with initial point $p$, will be called a forward horizontal [vertical] return trajectory at $p$. There is a natural map from the set of horizontal [vertical] return trajectories into the set of homotopically non-trivial closed curves. The closing of any horizontal [vertical] trajectory segment $\gamma_{ \pm q}^{\mathcal{T}}(p)$ is defined by

$$
\widehat{\gamma}_{ \pm q}^{\mathcal{T}}(p):=\gamma_{ \pm q}^{\mathcal{T}}(p) \cup \gamma\left(p, \Phi_{ \pm q}(p, \mathcal{T})\right)
$$

where $\gamma\left(p, \Phi_{ \pm q}(p, \mathcal{T})\right)$ is the shortest geodesic segment joining the endpoints $p, \Phi_{ \pm q}(p, \mathcal{T})$ of the trajectory segment (which exists by [58, Th. 18.2.2]).

Let $\mathcal{T}_{ \pm q}^{(1)}(p)$ be the forward horizontal [vertical] first return time of the $q$-regular point $p \in M$, defined to be the real number

$$
\mathcal{T}_{ \pm q}^{(1)}(p):=\min \left\{\mathcal{T}>0 \mid \Phi_{ \pm q}(p, \mathcal{T}) \in I_{\mp q}(p)\right\}
$$

The corresponding forward horizontal [vertical] trajectory $\gamma_{ \pm q}^{(1)}(p)$ with initial point $p$ will be called the forward horizontal [vertical] first return trajectory at $p$. We have the following estimate for first return times:

Lemma 9.2'. There exists a measurable function $K_{r}: \mathcal{M}_{\kappa} \rightarrow \mathbb{R}^{+}$such that, if $\mathcal{T}_{ \pm q}^{(1)}(p)$ is the forward horizontal [vertical] first return time of a $q$-regular point $p \in M$, then

$$
\|q\| / 2 \leq \mathcal{T}_{ \pm q}^{(1)}(p) \leq K_{r}(q) .
$$


Proof. Since $\widehat{\gamma}_{ \pm q}^{\mathcal{T}}(p)$ is a closed curve not homotopic to zero, there exists a shortest geodesic in its homotopy class [58, Th. 18.4.]. If such a geodesic contains singular points, then its length is $\geq\|q\|$. Otherwise, it is the waist curve of an embedded flat cylinder. Since the boundary of any flat cylinder is the union of saddle connections, the lower bound $\|q\|$ is valid also in this case. Since $I_{ \pm q}(p)$ has length $\|q\| / 2, \mathcal{T}_{ \pm q}^{(1)}(p) \geq\|q\| / 2$.

For all $q \in \mathcal{M}_{\kappa}$, the functions $p \rightarrow \mathcal{T}_{ \pm q}^{(1)}(p)$ are almost everywhere defined and simple, in the sense that they are locally constant and take only a finite set of values. In fact, if $I^{\mp}$ is an interval transverse to $\mathcal{F}_{ \pm q}$ of length $\delta>0$, the Poincaré return map on $I^{\mp}$ is an orientation-preserving isometry with (at most) a finite number of discontinuities, hence it is an interval exchange transformation with (at most) a finite number of sub-intervals. On each subinterval $\mathcal{T}_{ \pm q}^{(1)}(p)$ is constant. The function

$$
K_{r}(q):=\max \left\{\mathcal{T}_{ \pm q}^{(1)}(p) \mid p \in M \text { is } q \text {-regular }\right\}
$$

is therefore well-defined and everywhere finite. It can be proved that $K_{r}$ is lower semicontinuous, hence measurable, and (9.10) holds by the definition $\left(9.10^{\prime}\right)$.

9.3. Special sequences of 'close' return times. Special sequences of return times for the horizontal [vertical] foliation of a generic quadratic differential can be constructed by considering return times of the Teichmüller geodesic flow. This will clarify in what sense the Teichmüller flow $G_{t}$ and the cocycles $G_{t}^{K Z}, G_{t}^{c}$ play the role of a 'renormalization dynamics' for orientable measured foliations. Let $q \in \mathcal{M}_{\kappa}^{(1)}$ be a Birkhoff generic point of the Teichmüller flow $G_{t}$ and let $\mathcal{S}_{\kappa}(q) \subset \mathcal{M}_{\kappa}^{(1)}$ be a smooth compact hypersurface of codimension 1 , containing $q$ and transverse to the Teichmüller flow. Let $\left(t_{k}\right)$ be the sequence of return times of the orbit $\left\{G_{t}(q) \mid t \in \mathbb{R}\right\}$ to $\mathcal{S}_{\kappa}(q)$. Since, by definition,

$$
\left(\mathcal{F}_{q_{t}}, \mathcal{F}_{-q_{t}}\right)=\left(e^{-t} \mathcal{F}_{q}, e^{t} \mathcal{F}_{-q}\right)
$$

if $t=t_{k}<0$ is a backward return time of $G_{t}(q)$, any forward first return trajectory of the horizontal foliation $\mathcal{F}_{q_{t}}$ is a forward return trajectory of the horizontal foliation $\mathcal{F}_{q}$, provided $\left|t_{k}\right|$ is sufficiently large. In a similar way, if $t=t_{k}>0$ is a forward return time of $G_{t}(q)$, any forward first return trajectory of the vertical foliation $\mathcal{F}_{-q_{t}}$ is a forward return trajectory of the vertical foliation $\mathcal{F}_{-q}$. In all the arguments which follow we will consider the case of the horizontal foliation, the case of the vertical foliation being similar.

By closing the return trajectories of the horizontal [vertical] foliation, as in (9.8), we obtain closed currents of order 1 . The evolution of such currents under the action of the Teichmüller flow is therefore described by the cocycle $G_{t}^{c}$ 
on the bundle $\mathcal{Z}_{\kappa}^{1}(M)$, studied in Section 8.3 (in particular Theorem 8.7). We describe below a relevant additional property of the Oseledec's splitting (8.40).

In Theorem 8.7 we have proved that the Lyapunov spectrum of the cocycle $G_{t}^{c}$ on the bundle $\mathcal{E}_{\kappa}^{1}(M)$ of exact currents of order 1 is reduced to the single Lyapunov exponent 0 . In fact, there exists a Lyapunov norm on $\mathcal{E}_{\kappa}^{1}(M)$, invariant under $G_{t}^{c}$. Let $C$ be an exact current of order 1 , then $C=d U$, where the function $U \in L_{q}^{2}(M)$ has zero average.

Lemma 9.3. The norm $|\cdot|_{q}$, defined fiber-wise on the bundle $\mathcal{E}_{\kappa}^{1}(M)$ of exact currents of order 1 as

$$
|d U|_{q}:=|U|_{L_{q}^{2}(M)}, \quad \int_{M} U \omega_{q}=0,
$$

is invariant under $G_{t}^{c}$. There exists a continuous function $K: \mathcal{M}_{\kappa}^{(1)} \rightarrow \mathbb{R}^{+}$ such that, for all $q \in \mathcal{M}_{\kappa}^{(1)}$ and $d U \in \mathcal{E}_{q}^{1}(M)$, we have:

$$
K(q)|d U|_{q} \leq|d U|_{\mathcal{H}_{q}^{-1}(M)} \leq|d U|_{q}
$$

Proof. The norm $|d U|_{q}$ is invariant under the flow $G_{t}^{c}$, since $G_{t}^{c}(d U)=d U$ and the inner product of the Hilbert space $L_{q}^{2}(M)$ is invariant under the action of the Teichmüller flow $G_{t}$ on $\mathcal{M}_{\kappa}$. The second inequality in $\left(9.12^{\prime}\right)$ follows immediately from the definition (9.12).

Let $q \in \mathcal{M}_{\kappa}$ be a quadratic differential. By [18, Prop. 4.3A], the following splitting holds:

$$
H_{q}^{0}:=\left\{U \in L_{q}^{2}(M) \mid \int_{M} U \omega_{q}=0\right\}=R\left(\partial_{q}^{+}\right) \oplus R\left(\partial_{q}^{-}\right),
$$

where $R\left(\partial_{q}^{ \pm}\right) \subset L_{q}^{2}(M)$ is the range of the Cauchy-Riemann operator $\partial_{q}^{ \pm}$on the weighted Sobolev space $H_{q}^{1}(M)$. By [18, Prop. 3.2], the subspace $R\left(\partial_{q}^{+}\right)$ $\left[R\left(\partial_{q}^{-}\right)\right]$is the orthogonal complement in $L_{q}^{2}(M)$ of the finite-dimensional subspace $\mathcal{M}_{q}^{-}\left[\mathcal{M}_{q}^{+}\right]$of anti-meromorphic [meromorphic] functions. Since the Cauchy-Riemann operators $\partial_{q}^{ \pm}$depend continuously on the quadratic differential $q \in \mathcal{M}_{\kappa}$, there exists a continuous function $K_{1}: \mathcal{M}_{\kappa} \rightarrow \mathbb{R}^{+}$such that the following holds. If $U \in H_{q}^{0}$, then there exist functions $v^{ \pm} \in H_{q}^{1}(M)$ such that

$$
\begin{aligned}
U & =\partial_{q}^{+} v^{+}+\partial_{q}^{-} v^{-}, \\
\left|v^{ \pm}\right|_{1} & \leq K_{1}(q)|U|_{L_{q}^{2}(M)} .
\end{aligned}
$$

Hence

$$
\begin{aligned}
|U|_{0}^{2} & =\left(U, \partial^{+} v^{+}+\partial^{-} v^{-}\right)_{q} \\
& \leq 2|d U|_{-1}\left(\left|v^{+}\right|_{1}+\left|v^{-}\right|_{1}\right) \leq 4 K_{1}(q)|U|_{0}|d U|_{-1} .
\end{aligned}
$$


Let $q \in \mathcal{M}_{\kappa}^{(1)}$ be a regular point for the Kontsevich-Zorich cocycle (in the sense of the Oseledec's theorem). Let

$$
\lambda_{1}^{\prime}=1>\lambda_{2}^{\prime}>\cdots \lambda_{s}^{\prime}>0>-\lambda_{s}^{\prime}>\cdots>-\lambda_{1}^{\prime}=-1
$$

be the distinct Lyapunov exponents at $q$ of the Kontsevich-Zorich cocycle. By Corollary 2.2 and Theorem 8.5, there exists $s \in\{2, \ldots, g\}$ such that (9.15) holds. It has been conjectured in [38], on the basis on numerical evidence, that the Lyapunov spectrum of the Kontsevich-Zorich cocycle is simple, hence $s=g$. By Theorem 8.7, there exists an Oseledec's splitting

$$
\mathcal{Z}_{q}^{1}(M)=E_{1}^{+}(q) \oplus \cdots \oplus E_{s}^{+}(q) \bigoplus E_{1}^{-}(q) \oplus \cdots \oplus E_{s}^{-}(q) \bigoplus \mathcal{E}_{q}^{1}(M),
$$

where $E_{i}^{ \pm}(q), i \in\{1, \ldots s\}$, is the eigenspace of the cocycle $G_{t}^{c}$ corresponding to the Lyapunov exponent $\pm \lambda_{i}^{\prime}$ and $\mathcal{E}_{q}^{1}(M)$ is the closed infinite-dimensional subspace of exact currents of degree 1 . The subspaces $E_{i}^{ \pm}(q)$ are finite dimensional with dimension equal to the multiplicity of the Lyapunov exponent $\lambda_{i}^{\prime}$, while the subspace $\mathcal{E}_{q}^{1}(M)$ is infinite-dimensional.

Let $\Pi_{q}^{ \pm i}: \mathcal{Z}_{q}^{1}(M) \rightarrow E_{i}^{ \pm}(q), i \in\{1, \ldots, s\}, \Pi_{q}^{\mathcal{E}}: \mathcal{Z}_{q}^{1}(M) \rightarrow \mathcal{E}_{q}^{1}(M)$ be the projections determined by the splitting $\left(9.15^{\prime}\right)$. Such projections can be extended to the (Hilbert) space $\mathcal{H}_{q}^{-1}(M)$ by composition with the orthogonal projection onto the closed subspace $\mathcal{Z}_{q}^{1}(M)$. Let $\delta_{\kappa}: \mathcal{M}_{\kappa}^{(1)} \rightarrow \mathbb{R}^{+}$be the distorsion (in the sense of [41, Chap. IV, §11, p. 269]) of the splitting $\left(9.15^{\prime}\right)$ with respect to the $\mathcal{H}_{q}^{-1}$ norm on $\mathcal{B}_{q}^{1}(M) \oplus \mathcal{M}_{-q}^{1}(M)$ and the Lyapunov norm $|\cdot|_{q}$ (introduced in Lemma 9.3) on $\mathcal{E}_{q}^{1}(M)$ :

$$
\delta_{\kappa}(q):=\sup _{C} \frac{\sum_{i=1}^{g}\left|\Pi_{q}^{+i}(C)\right|_{-1}^{2}+\sum_{i=1}^{g}\left|\Pi_{q}^{-i}(C)\right|_{-1}^{2}+\left|\Pi_{q}^{\mathcal{E}}(C)\right|_{q}^{2}}{|C|_{-1}^{2}}
$$

where the supremum is taken over all currents $C \in \mathcal{Z}_{q}^{1}(M)$.

Let $\mathcal{P}_{\kappa}^{(1)} \subset \mathcal{M}_{\kappa}^{(1)}$ be a compact set satisfying the following conditions:

(1) All $q \in \mathcal{P}_{\kappa}^{(1)}$ are Birkhoff generic points for the Teichmüller flow $G_{t}$ and Oseledec regular points for the cocycle $G_{t}^{c}$;

(2) $\mathcal{P}_{\kappa}^{(1)}$ is transverse to the Teichmüller flow and has positive transverse measure;

(3) the distorsion $\delta_{\kappa}$, defined by $\left(9.15^{\prime \prime}\right)$, is continuous (hence bounded) on $\mathcal{P}_{\kappa}^{(1)}$

(4) the function $K_{r}: \mathcal{M}_{\kappa}^{(1)} \rightarrow \mathbb{R}^{+}$, that is the upper bound (9.10) on the first return times of the horizontal and vertical foliations, is continuous (hence bounded) on $\mathcal{P}_{\kappa}^{(1)}$. 
It follows from the ergodicity of the Teichmüller flow (Theorem 1.1), from Theorem 8.7, Lemma $9.2^{\prime}$ and Luzin's theorem that the union of all sets $\mathcal{P}_{\kappa}^{(1)}$ with the properties (1)-(4) is a full measure subset of $\mathcal{M}_{\kappa}^{(1)}$.

Let $q \in \mathcal{P}_{\kappa}^{(1)}$ and $\left(t_{k}\right)_{k \in \mathbb{N}}$ be the sequence of return times of the backward orbit $\left\{G_{t}(q) \mid t \leq t_{1}=0\right\}$ to $\mathcal{P}_{\kappa}^{(1)}$. Let $p \in M$ be any $q$-regular point. A principal sequence of forward return times at $p$ for the horizontal foliation $\mathcal{F}_{q}$ is the sequence

$$
\mathcal{T}_{q}^{(k)}(p):=\mathcal{T}_{G_{t}(q)}^{(1)}(p) \exp |t|, \quad t=t_{k} .
$$

The horizontal forward trajectory at $p$ corresponding to a principal return time $\mathcal{T}_{q}^{(k)}(p)$ will be called a horizontal principal return trajectory at $p$ and denoted by $\gamma_{q}^{(k)}(p)$. We remark that a horizontal principal return trajectory $\gamma_{q}^{(k)}(p)$ coincides with the horizontal first return trajectory at $p$ of the quadratic differential $G_{t}(q), t=t_{k}<0$. A similar construction holds for the vertical foliation $\mathcal{F}_{-q}$ by considering forward return times of the Teichmüller flow.

Lemma 9.4. Under the conditions (1)-(4) there exists a constant $K_{\mathcal{P}}>0$ such that the following holds. Let $q \in \mathcal{P}_{\kappa}^{(1)}$ and $\gamma_{q}^{\mathcal{T}}(p), \mathcal{T}>0$, be a forward trajectory with initial point a q-regular point $p \in M$. There exists a finite sequence of points $\left(p_{j}^{(k)}\right) \subset \gamma_{q}^{\mathcal{T}}(p), 1 \leq k \leq n, 1 \leq j \leq m_{k}$, such that the principal return trajectories $\gamma_{q}^{(k)}\left(p_{j}^{(k)}\right) \subset \gamma_{q}^{\mathcal{T}}(p)$ do not overlap and, in addition,

$$
\begin{aligned}
& \gamma_{q}^{\mathcal{T}}(p)=\sum_{k=1}^{n} \sum_{j=1}^{m_{k}} \gamma_{q}^{(k)}\left(p_{j}^{(k)}\right)+b_{q}^{\mathcal{T}}(p) \\
& m_{k} \leq K_{\mathcal{P}} \exp \left(\left|t_{k+1}\right|-\left|t_{k}\right|\right), \\
& L_{q}\left(b_{q}^{\mathcal{T}}(p)\right) \leq K_{\mathcal{P}} .
\end{aligned}
$$

Proof. The proof is based on the following estimate on principal return times. By (9.16), Lemma 9.2' and condition (4), there exists a constant $K_{p r}>0$ such that, for all $q \in \mathcal{P}_{\kappa}^{(1)}$, all $q$-regular points $p \in M$ and all $k \in \mathbb{N}$,

$$
K_{p r}^{-1} \exp \left|t_{k}\right| \leq \mathcal{T}_{q}^{(k)}(p) \leq K_{p r} \exp \left|t_{k}\right| .
$$

Let $n=\max \left\{k \in \mathbb{N} \mid \mathcal{T}_{q}^{(k)}(p) \leq \mathcal{T}\right\}$. The maximum exists (i.e., is finite) by (9.17). Let $p_{1}^{(n)}:=p$. The sequence $\left(p_{j}^{(k)}\right)$ with the properties stated in $\left(9.16^{\prime}\right)$ can be constructed by a finite iteration of the following procedure. Let $p_{j}^{(k)}$ be the last point in the sequence already determined and let

$$
p_{j+}^{(k)}:=\Phi_{q}\left(p_{j}^{(k)}, \mathcal{T}_{q}^{(k)}\left(p_{j}^{(k)}\right)\right) \in \gamma_{q}^{\mathcal{T}}(p) .
$$


Let then $k^{\prime} \in\{1, \ldots, k\}$ be the largest integer such that

$$
\Phi_{q}\left(p_{j+}^{(k)}, \mathcal{T}_{q}^{\left(k^{\prime}\right)}\left(p_{j+}^{(k)}\right)\right) \in \gamma_{q}^{\mathcal{T}}(p) .
$$

If $k^{\prime}=k$, let $p_{j+1}^{(k)}:=p_{j+}^{(k)}$. If $k^{\prime}<k$, let $m_{k}:=j, m_{h}=0$ (no points) for all $k^{\prime}<h<k$ and $p_{1}^{\left(k^{\prime}\right)}:=p_{j+}^{(k)}$. The iteration step is concluded. By (9.17) we have:

$$
K_{p r}^{-1} \exp \left|t_{k}\right| m_{k} \leq \mathcal{T}_{q}^{(k+1)}\left(p_{1}^{(k)}\right) \leq K_{p r} \exp \left|t_{k+1}\right| .
$$

The length of the remainder $b_{q}^{\mathcal{T}}(p)$ has to be less than any upper bound for the length of first return times. Hence, by (9.19), Lemma 9.2 ${ }^{\prime}$ and condition (4), the estimates in $\left(9.16^{\prime}\right)$ are proved and the argument is concluded.

\subsection{The main theorem on the deviation of ergodic averages.}

THEOREM 9.5. For almost all quadratic differentials $q \in \mathcal{M}_{\kappa}^{(1)}$ and almost all points $p \in M_{q}$ the following holds. Let $\gamma_{q}^{\mathcal{T}}(p)$ be the horizontal forward trajectory segment of length $\mathcal{T}>0$ with initial point $p$. Then, for all $i \in$ $\{1, \ldots s\}$

$$
\begin{aligned}
& \limsup _{\mathcal{T} \rightarrow+\infty} \frac{\log \left|\Pi_{q}^{+i}\left(\gamma_{q}^{\mathcal{T}}(p)\right)\right|_{-1}}{\log \mathcal{T}}=\lambda_{i}^{\prime}, \\
& \limsup _{\mathcal{T} \rightarrow+\infty} \frac{\log \left|\Pi_{q}^{-i}\left(\gamma_{q}^{\mathcal{T}}(p)\right)\right|_{-1}}{\log \mathcal{T}}=0 \\
& \limsup _{\mathcal{T} \rightarrow+\infty} \frac{\log \left|\Pi_{q}^{\mathcal{E}}\left(\gamma_{q}^{\mathcal{T}}(p)\right)\right|_{-1}}{\log \mathcal{T}}=0 .
\end{aligned}
$$

Proof. The argument consists of two parts. We first prove the upper bounds implicit in (9.20), then the lower bound.

Upper bound. By Lemma 9.4 the estimate can be reduced to the case of a principal return trajectory $\gamma_{q}^{(k)}(p)$. Let $\mathcal{P}_{\kappa}^{(1)}$ be a compact set satisfying conditions (1)-(4) listed in Section 9.3. By Lemmas 9.2, 9.2' and the conditions (3), (4), there exists a constant $K_{1}>0$ such that the following holds. Let $q \in \mathcal{P}_{\kappa}^{(1)}$ and $p \in M$ be a $q$-regular point. Let $\gamma_{q}^{(1)}(p)$ be the first return horizontal trajectory at $p$. Then

$$
\begin{gathered}
\left|\Pi_{q}^{ \pm i}\left(\widehat{\gamma}_{q}^{(1)}(p)\right)\right|_{-1} \leq K_{1}, \\
\left|\Pi_{q}^{\mathcal{E}}\left(\widehat{\gamma}_{q}^{(1)}(p)\right)\right|_{q} \leq K_{1},
\end{gathered}
$$

where the closing operation $\gamma \rightarrow \widehat{\gamma}$ of a return trajectory is described by (9.8) and $|\cdot|_{q}$ is the Lyapunov norm on $\mathcal{E}_{q}^{1}(M)$ defined in Lemma 9.3. The currents 
of order 1 given by the principal return trajectories can be obtained by the action of the cocycle $G_{t}^{c}$ on the first return trajectories:

$$
\gamma_{q}^{(k)}(p)=G_{-t}^{c}\left(\gamma_{G_{t}(q)}^{(1)}(p)\right), t=t_{k} \leq 0
$$

where $\left(t_{k}\right)$ is the sequence of backward return times of the Teichmüller orbit $G_{t}(q) \mathcal{P}_{\kappa}^{(1)}$. Hence, by Theorem 8.7, Lemma 9.3 and the invariance of the Lyapunov norm $|\cdot|_{q}$ under the cocycle $G_{t}^{c}$, if $\lambda_{i}^{+}>\lambda_{i}^{\prime}>0$ and $-\lambda_{i}^{\prime}<\lambda_{i}^{-}<0$, there exists a constant $K_{2}>0$, such that

$$
\begin{aligned}
& \left|\Pi_{q}^{ \pm i}\left(\widehat{\gamma}_{q}^{(k)}(p)\right)\right|_{-1} \leq K_{2} \exp \left(\lambda_{i}^{ \pm}\left|t_{k}\right|\right), \\
& \left|\Pi_{q}^{\mathcal{E}}\left(\widehat{\gamma}_{q}^{(k)}(p)\right)\right|_{-1} \leq K_{2} .
\end{aligned}
$$

By Lemma 9.4 the trajectory $\gamma_{q}^{\mathcal{T}}(p)$ can be split as a union of principal return trajectories and a uniformly bounded remainder. Hence, by Lemma 9.2, (9.11) and $\left(9.22^{\prime}\right)$, there exists a constant $K_{3}>0$ such that

$$
\begin{aligned}
& \left|\Pi_{q}^{ \pm i}\left(\gamma_{q}^{\mathcal{T}}(p)\right)\right|_{-1} \leq K_{3} \sum_{1 \leq k \leq n} m_{k} \exp \left(\lambda_{i}^{ \pm}\left|t_{k}\right|\right), \\
& \left|\Pi_{q}^{\mathcal{E}}\left(\gamma_{q}^{\mathcal{T}}(p)\right)\right|_{-1} \leq K_{3} \sum_{1 \leq k \leq n} m_{k} .
\end{aligned}
$$

Since the transverse set $\mathcal{P}_{\kappa}^{(1)}$ has positive transverse measure, by the ergodicity of the Teichmüller flow (Theorem 1.1) and Birkhoff ergodic theorem, the backward return times $\left(t_{k}\right)$ have the following property:

$$
\lim _{k \rightarrow+\infty} \frac{\left|t_{k}\right|}{k}=\frac{1}{\mu}
$$

where $\mu:=\mu\left(\mathcal{P}_{\kappa}^{(1)}\right)>0$ is the transverse measure of $\mathcal{P}_{\kappa}^{(1)}$. Let $0<\delta \ll 1 / \mu$. By (9.23) there exists $N_{\delta} \in \mathbb{N}$ such that, for all $k \geq N_{\delta}$,

$$
\left(\frac{1}{\mu}-\delta\right) k \leq\left|t_{k}\right| \leq\left(\frac{1}{\mu}+\delta\right) k,
$$

hence, by the estimates proved in Lemma 9.4 and $\left(9.23^{\prime}\right)$, there exist constants $K_{\mu, \delta}^{(1)}, K_{\mu, \delta}^{(2)}>0$ such that, for all $n>N_{\delta}$,

$$
\begin{aligned}
& \left|\Pi_{q}^{ \pm i}\left(\gamma_{q}^{\mathcal{T}}(p)\right)\right|_{-1} \leq K_{\mu, \delta}^{(1)}+K_{\mu, \delta}^{(2)}\left|\exp \left\{\left(\frac{\lambda_{i}^{ \pm}}{\mu}+\left(\lambda_{i}^{ \pm}+2\right) \delta\right) n\right\}-1\right|, \\
& \left|\Pi_{q}^{\mathcal{E}}\left(\gamma_{q}^{\mathcal{T}}(p)\right)\right|_{-1} \leq K_{\mu, \delta}^{(1)}+K_{\mu, \delta}^{(2)} \exp (2 \delta n) .
\end{aligned}
$$

On the other hand, by the choice of $n \in \mathbb{N}$ in Lemma 9.4,

$$
n=\max \left\{k \in \mathbb{N} \mid \mathcal{T}_{q}^{(k)}(p) \leq \mathcal{T}_{r}\right\},
$$


hence, by (9.16) and Lemma $9.2^{\prime}$, there exists a constant $K_{4}>0$ such that

$$
K_{4} \exp \left\{\left(\frac{1}{\mu}-\delta\right) n\right\} \leq K_{4} \exp \left(\left|t_{n}\right|\right) \leq \mathcal{T} .
$$

By $(9.24)$ and $\left(9.24^{\prime}\right)$,

$$
\begin{aligned}
& \limsup _{\mathcal{T} \rightarrow+\infty} \frac{\log \left|\Pi_{q}^{+i}\left(\gamma_{q}^{\mathcal{T}}(p)\right)\right|_{-1}}{\log \mathcal{T}} \leq \frac{\lambda_{i}^{+}+\left(\lambda_{i}^{+}+2\right) \mu \delta}{1-\mu \delta} \\
& \limsup _{\mathcal{T} \rightarrow+\infty} \frac{\log \left|\Pi_{q}^{-i}\left(\gamma_{q}^{\mathcal{T}}(p)\right)\right|_{-1}}{\log \mathcal{T}} \leq \frac{\max \left\{0, \lambda_{i}^{-}+\left(\lambda_{i}^{-}+2\right) \mu \delta\right\}}{1-\mu \delta} \\
& \limsup _{\mathcal{T} \rightarrow+\infty} \frac{\log \left|\Pi_{q}^{\mathcal{E}}\left(\gamma_{q}^{\mathcal{T}}(p)\right)\right|_{-1}}{\log \mathcal{T}} \leq \frac{2 \mu \delta}{1-\mu \delta}
\end{aligned}
$$

Since $0<\delta \ll 1 / \mu$ and $\lambda_{i}^{+}>\lambda_{i}^{\prime}>0, \lambda_{i}^{-}<-\lambda_{i}^{\prime}<0$ are arbitrarily chosen, the upper bound in (9.20) is proved.

Lower bound. We claim the following statement holds. For $\mu_{\kappa}^{(1)}$-almost all quadratic differentials $q \in \mathcal{M}_{\kappa}^{(1)}$, there exists a full measure set $A_{q} \subset M_{q}$ (consisting of $q$-regular points) with the following property. For each $p \in A_{q}$, there exists a diverging sequence $\mathcal{T}_{k}:=\mathcal{T}_{k}(p)$ of positive times such that

$$
\lim _{k \rightarrow+\infty} \frac{\log \left|\Pi_{q}^{+i}\left(\gamma_{q}^{\mathcal{T}_{k}}(p)\right)\right|_{-1}}{\log \mathcal{T}_{k}}=\lambda_{i}^{\prime}
$$

We remark that it is sufficient to find a positive measure set $\mathcal{P}_{\kappa}^{(1)}$ in every connected component of the moduli space $\mathcal{M}_{\kappa}^{(1)}$ with the property that (9.26) holds on a set $A_{q} \subset M_{q}$ of positive measure. In fact, since almost all quadratic differentials have ergodic horizontal foliation [43], if (9.26) holds on a positive measure subset of $M_{q}$, it holds on a full measure subset. Then, by the ergodicity of the Teichmüller flow on each connected component of the moduli space (Theorem 1.1), if (9.26) holds for almost all $p \in M_{q}$ on a positive measure subset $\mathcal{P}_{\kappa}^{(1)}$ of a connected component $\mathcal{C}_{\kappa}^{(1)} \subset \mathcal{M}_{\kappa}^{(1)}$, then it holds on a full measure subset of $\mathcal{C}_{\kappa}^{(1)}$.

Let $q_{0} \in \mathcal{C}_{\kappa}^{(1)}$ be a quadratic differential with Lagrangian horizontal foliation satisfying the properties listed in Lemma 8.4. The flat structure induced by $q_{0}$ contains at least $g \geq 2$ embedded flat cylinders $A_{j}^{(0)}$ with waist curves $\gamma_{j}^{(0)}, j \in\{1, \ldots, g\}$, such that the subspace $\mathcal{L}_{0} \subset H_{1}\left(M_{q_{0}}, \mathbb{R}\right)$ generated by $\left\{\gamma_{1}^{(0)}, \ldots, \gamma_{g}^{(0)}\right\}$ is Lagrangian. Let $h_{j}>0$ be the height of the cylinder $A_{j}^{(0)}$. Then $A_{j}^{(0)} \equiv \gamma_{j}^{(0)} \times\left(-h_{j} / 2, h_{j} / 2\right)$. There exists a compact neighbourhood $\mathcal{U}_{\kappa}^{(1)}$ of $q_{0}$ in $\mathcal{C}_{\kappa}^{(1)}$ such that all $q \in \mathcal{U}_{\kappa}^{(1)}$ have embedded flat cylinders $A_{j}(q)$ with waist curves isotopic to $\gamma_{j}^{(0)}$ and, in addition, the following two properties hold: 
(1) the closing $\widehat{\gamma}_{q}^{(1)}(p)$ of the $q$-horizontal first return trajectory of any point $p \in A_{j}(q)$ is isotopic to a waist curve of $A_{j}(q)$ in $M_{q} \backslash \Sigma_{q}$;

(2) there is a strictly positive lower bound for the area of all cylinders $A_{j}(q) \subset$ $M_{q}, q \in \mathcal{U}_{\kappa}^{(1)}$.

Let $\mathcal{P}_{\kappa}^{(1)} \subset \mathcal{U}_{\kappa}^{(1)}$ be a compact positive measure set satisfying the conditions listed in Lemma 8.4 and, in addition, the conditions (1) - (4) listed in Section 9.3. Since, by $(2 \mathrm{c})$ in Lemma 8.4, the Poincaré dual $P\left(\mathcal{L}_{0}\right)$ of the Lagrangian subspace $\mathcal{L}_{0}$, generated by the waist curves $\left\{\gamma_{1}^{(0)}, \ldots, \gamma_{g}^{(0)}\right\}$, is transverse to the stable subspace $E^{-}(q)$, the condition (1) above implies the following. Let $q \in \mathcal{P}_{\kappa}^{(1)}$ and $\Pi_{q}^{ \pm i}, i \in\{1, \ldots, s\}$, be one of the projections determined by the Oseledec splitting $\left(9.15^{\prime}\right)$. There exists a cylinder $A_{j(i)}(q)$ such that

$$
\Pi_{q}^{i}\left(\widehat{\gamma}_{q}^{(1)}(p)\right) \neq 0, \quad \text { for all } p \in A_{j(i)}(q) .
$$

By Luzin's theorem, conditions (3), (4) in Section 9.3, Lemmas 9.2, 9.2' and (9.27), there exist a compact positive measure set $\mathcal{P}_{\kappa}^{\prime} \subset \mathcal{P}_{\kappa}^{(1)}$, on which the splitting $\left(9.15^{\prime}\right)$ depends continuously on the quadratic differential, and a constant $K_{5}>0$ such that, if $q \in \mathcal{P}_{\kappa}^{\prime}$, we have:

$$
K_{5}^{-1} \leq\left|\Pi_{q}^{i}\left(\widehat{\gamma}_{q}^{(1)}(p)\right)\right|_{-1} \leq K_{5}, \quad p \in A_{j(i)}(q) .
$$

Let $q \in \mathcal{P}_{\kappa}^{\prime}$ and $\left(t_{n}\right)$ be the sequence of backward return times of the orbit $G_{t}(q)$ to the positive measure set $\mathcal{P}_{\kappa}^{\prime}$. Let $A_{j(i)}^{(n)}:=A_{j(i)}\left(q_{n}\right), q_{n}:=G_{t_{n}}(q)$. Let $p \in A_{j(i)}^{(n)}$ and $\widehat{\gamma}_{q}^{(n)}(p)$ be the closing of the first return trajectory at $p$ of the horizontal foliation of $G_{t_{n}}(q)$. Then, by Theorem 8.7, for any $\lambda_{i}^{-}<\lambda_{i}<\lambda^{+}$, there is a constant $K_{6}>0$ such that

$$
K_{6}^{-1} \exp \left(\lambda_{i}^{-}\left|t_{n}\right|\right) \leq\left|\Pi_{q}^{i}\left(\widehat{\gamma}_{q}^{(n)}(p)\right)\right|_{-1} \leq K_{6} \exp \left(\lambda_{i}^{+}\left|t_{n}\right|\right) .
$$

Let $\mathcal{T}_{q}^{(n)}(p)$ be the return time of the return trajectory $\gamma_{q}^{(n)}(p), p \in A_{j(i)}^{(n)}$. By (9.17) there exists a constant $K_{p r}>0$ such that, for all $q$-regular $p \in M_{q}$,

$$
K_{p r}^{-1} \exp \left|t_{n}\right| \leq \mathcal{T}_{q}^{(n)}(p) \leq K_{p r} \exp \left|t_{n}\right| .
$$

All cylinders $A_{j(i)}^{(n)}$ have positive area in $M_{q}$, uniformly bounded away from zero (as a consequence of the corresponding property of the cylinders $A_{j(i)}(q)$ for all $q \in \mathcal{U}_{\kappa}^{(1)}$ and of the invariance of the area form $\omega_{q}$ under the Teichmüller flow). Hence the set

$$
A_{q}:=\bigcap_{N \in \mathbb{N}} \bigcup_{n \geq N} A_{j(i)}^{(n)}
$$

such that $p \in A_{q}$ if and only if $p \in A_{j(i)}^{(n)}$ for infinitely many $n \in \mathbb{N}$, has positive measure in $M_{q}$. In fact, the $R_{q}$-area of $A_{q}$ is greater than any lower bound for 
the $R_{q}$-area of the sets $A_{j(i)}^{(n)}$. This is a simple instance of the Kochen-Stone inequality $\left[19, \S 6.2\right.$, Lemma 4]. By $\left(9.28^{\prime}\right)$ and $\left(9.28^{\prime \prime}\right)$, for each $p \in A_{q}$ there exists therefore a diverging sequence $\left(n_{k}\right)$ such that

$$
\lim _{k \rightarrow+\infty} \frac{\log \left|\Pi_{q}^{+i}\left(\gamma_{q}^{\left(n_{k}\right)}(p)\right)\right|_{-1}}{\log \mathcal{T}^{\left(n_{k}\right)}}=\lambda_{i}^{\prime} .
$$

Let $\mathcal{T}_{k}(p):=\mathcal{T}^{\left(n_{k}\right)}$. We have proved (9.26) for a positive measure set $\mathcal{P}_{\kappa}^{\prime}$ of quadratic differentials in each connected component of the stratum $\mathcal{M}_{\kappa}^{(1)}$ and, given any $q \in \mathcal{P}_{\kappa}^{\prime}$, for a positive measure set $A_{q} \subset M_{q}$. By the above remark the argument is concluded.

Remark 9.5'. In proving Theorem 9.5, we have in fact proved the following statement. For almost all $q \in \mathcal{M}_{\kappa}^{(1)}$ and any given $\varepsilon>0$ there exists $\mathcal{T}_{\varepsilon}:=$ $\mathcal{T}_{\varepsilon}(q)>0$ such that, for all $i \in\{1, \ldots, s\}$ and all $q$-regular $p \in M_{q}$,

$$
\begin{aligned}
& \sup _{\mathcal{T} \geq \mathcal{T}_{\varepsilon}} \frac{\log \left|\Pi_{q}^{+i}\left(\gamma_{q}^{\mathcal{T}}(p)\right)\right|_{-1}}{\log \mathcal{T}} \leq \lambda_{i}^{\prime}+\varepsilon, \\
& \sup _{\mathcal{T} \geq \mathcal{T}_{\varepsilon}} \frac{\log \left|\Pi_{q}^{-i}\left(\gamma_{q}^{\mathcal{T}}(p)\right)\right|_{-1}}{\log \mathcal{T}} \leq \varepsilon, \\
& \sup _{\mathcal{T} \geq \mathcal{T}_{\varepsilon}} \frac{\log \left|\Pi_{q}^{\mathcal{E}}\left(\gamma_{q}^{\mathcal{T}}(p)\right)\right|_{-1}}{\log \mathcal{T}} \leq \varepsilon .
\end{aligned}
$$

In addition, for each $i \in\{1, \ldots, s\}$, there exist a cylinder $A_{\varepsilon}^{(i)}(q) \subset M_{q}$, with $\operatorname{vol}_{q}\left(A_{\varepsilon}^{(i)}(q)\right)$ uniformly bounded away from zero (independently of $\varepsilon>0$ ), and a real number $\mathcal{T}_{\varepsilon}^{(i)}:=\mathcal{T}_{\varepsilon}^{(i)}(q)>0$ such that, for all $p \in A_{\varepsilon}^{(i)}(q)$,

$$
\frac{\log \left|\Pi_{q}^{+i}\left(\gamma_{q}^{\mathcal{T}_{\varepsilon}^{(i)}}(p)\right)\right|_{-1}}{\log \mathcal{T}_{\varepsilon}^{(i)}} \geq \lambda_{i}^{\prime}-\varepsilon
$$

and there exists a constant $K_{q}^{(i)}>0$ such that, for all $p_{1}, p_{2} \in A_{\varepsilon}^{(i)}(q)$,

$$
\left|\Pi_{q}^{+i}\left(\gamma_{q}^{\mathcal{T}_{\varepsilon}^{(i)}}\left(p_{1}\right)\right)-\Pi_{q}^{+i}\left(\gamma_{q}^{\mathcal{T}_{\varepsilon}^{(i)}}\left(p_{2}\right)\right)\right|_{-1} \leq K_{q}^{(i)} .
$$

The bound in $\left(9.32^{\prime}\right)$ follows from the remark that all return trajectories $\widehat{\gamma}_{q}^{\mathcal{T}_{\varepsilon}^{(i)}}(p), p \in A_{\varepsilon}^{(i)}(q)$, are isotopic in $M_{q} \backslash \Sigma_{q}$ to the waist curve of a flat cylinder (of uniformly bounded area).

The argument also proves that the cohomology class of the closed current $\widehat{\gamma}_{q}^{\mathcal{T}}(p)$ stays within a bounded distance of the unstable subspace $E_{q}^{+}$of the Kontsevich-Zorich cocycle in the cohomology group $H^{1}(M, \mathbb{R})$. In fact, by choosing $\delta>0$ sufficiently small in (9.24), we obtain the following estimate. 
There is a constant $K_{q}>0$ such that, for all $i \in\{1, \ldots, s\}$ and all $q$-regular points $p \in M_{q}$,

$$
\sup _{\mathcal{T}>0}\left|\Pi_{q}^{-i}\left(\hat{\gamma}_{q}^{\mathcal{T}}(p)\right)\right|_{-1} \leq K_{q}
$$

9.5. The deviation for area-preserving vector fields and open questions. Let $\omega$ be a smooth area form on a compact orientable surface $M$ of genus $g \geq 2$ with at most finite order degeneracies at a finite set $\Sigma:=\left\{p_{1}, \ldots, p_{\sigma}\right\} \subset M$. Let $\imath:=\left(\imath_{1}, \ldots, \imath_{\sigma}\right)$ satisfy the following properties: each $\imath_{k}$ is a negative integer and $\sum \imath_{k}=2-2 g$. Let $\mathcal{E}_{\omega}^{\imath}(M, \Sigma)$ the set of vector fields $X$ such that the closed 1-form $\eta_{X}:=\imath_{X} \omega$ is smooth on $M$ and the orbit foliation $\mathcal{F}_{X}:=\left\{\eta_{X}=0\right\}$ is a measured foliation in the sense of [62] with a saddle-type singularity of index $\imath_{k}$ at each point $p_{k} \in \Sigma, k=1, \ldots, \sigma$. The measure class on $\mathcal{E}_{\omega}^{\imath}(M, \Sigma)$ is defined by pull-back of Lebesgue measure class under the map $\mathcal{E}_{\omega}^{\imath}(M, \Sigma) \rightarrow H^{1}(M, \Sigma ; \mathbb{R})$ given by the Katok's fundamental class [29]:

$$
X \rightarrow\left[\eta_{X}\right] \in H^{1}(M, \Sigma ; \mathbb{R}) .
$$

For almost all $X \in \mathcal{E}_{\omega}^{\imath}(M, \Sigma)$, the orbit foliation $\mathcal{F}_{X}$ can be realized as the horizontal foliation of an orientable quadratic differential $q \in Q_{\kappa}^{(1)}$ [27, Chap. II] with $\kappa=-2$. Let $\mathcal{C}_{X}^{(1)}$ be the connected component of the stratum $\mathcal{M}_{\kappa}^{(1)}$ of the moduli space such that $q \in \mathcal{C}_{X}^{(1)}$ and let

$$
\left\{\left(\lambda_{i}^{\prime}(X), m_{i}(X)\right) \mid i=1, \ldots, s\right\} \subset \mathbb{R}^{+} \times \mathbb{N} \backslash\{0\}
$$

be the positive Lyapunov spectrum (with multiplicities) of the KontsevichZorich cocycle on the connected component $\mathcal{C}_{X}^{(1)}$. The distinct Lyapunov exponents $\lambda_{1}^{\prime}(X)=1>\lambda_{2}^{\prime}(X)>\cdots>\lambda_{s}^{\prime}(X)>0$ and the corresponding multiplicities $m_{1}(X)=1, \ldots, m_{s}(X)$ (such that $\sum m_{i}(X)=g$ ) are defined for almost all $X \in \mathcal{E}_{\omega}^{\imath}(M, \Sigma)$ and only depend on the connected component $\mathcal{C}_{X}^{(1)}$. The Kontsevich-Zorich conjecture (1.2) states that $m_{i}(X)=1$ for all $i \in\{1, \ldots, s\}$ (hence $s=g$ ). We have proved only the weaker statement that all the Lyapunov exponents are non-zero (Theorem 8.5).

If $X \in \mathcal{E}_{\omega}^{\imath}(M, \Sigma)$ and $\mathcal{F}_{X}=\mathcal{F}_{q}$, there exists a strictly positive smooth function $W=W_{X}$, with zeroes of finite order at $\Sigma$, such that $\omega=W^{-1} \omega_{q}$, hence $X=W S$, where $S$ is the normalized horizontal vector field for the metric $R_{q}$, defined in Section 2, and $\omega_{q}$ is the area form of $R_{q}$. Let

$$
H_{W}^{1}(M):=\left\{f \mid W^{-1} f \in H^{1}(M)\right\}
$$

be the related weighted Sobolev space of once weakly differentiable functions. 
TheOREM 9.6. For almost all vector fields $X \in \mathcal{E}_{\omega}^{\imath}(M, \Sigma)$, the vector space $\mathcal{I}_{X}^{1}(M)$ of $X$-invariant distributions which belong to the dual weighted Sobolev space $H_{W}^{-1}(M)$ (i.e. solutions $\mathcal{D}^{X} \in H_{W}^{-1}(M)$ of the equation $S \mathcal{D}=0$ ) has precisely dimension $g \geq 2$. There exists a splitting

$$
\mathcal{I}_{X}^{1}(M)=\mathcal{I}_{X}^{1}\left(\lambda_{1}^{\prime}\right) \oplus \mathcal{I}_{X}^{1}\left(\lambda_{2}^{\prime}\right) \oplus \cdots \oplus \mathcal{I}_{X}^{1}\left(\lambda_{s}^{\prime}\right),
$$

where the dimension of the subspace $\mathcal{I}_{X}^{1}\left(\lambda_{i}^{\prime}\right)$ is equal to the multiplicity $m_{i}(X)$ of the $i^{\text {th }}$ Lyapunov exponent $\lambda_{i}^{\prime}(X)>0$ of the Kontsevich-Zorich cocycle on the connected component $\mathcal{C}_{X}^{(1)}$, such that the following holds. Let $\Phi_{X}^{\mathcal{T}}$ denote the flow of the vector field $X$. Let $f \in H_{W}^{1}(M)$ be any function such that

$$
\mathcal{D}^{X}(f)=0, \quad \text { for all } \mathcal{D}^{X} \in \mathcal{I}_{X}^{1}\left(\lambda_{1}^{\prime}\right) \oplus \mathcal{I}_{X}^{1}\left(\lambda_{2}^{\prime}\right) \oplus \cdots \oplus \mathcal{I}_{X}^{1}\left(\lambda_{i}^{\prime}\right),
$$

then, if $0 \leq i<s$, for all $p \in M$ with regular forward trajectory,

$$
\limsup _{\mathcal{T} \rightarrow+\infty} \frac{\log \left|\int_{0}^{\mathcal{T}} f\left(\Phi_{X}(p, \tau)\right) d \tau\right|}{\log \mathcal{T}} \leq \lambda_{i+1}^{\prime}(X),
$$

and there exists $\mathcal{D}_{i+1}^{X} \in \mathcal{I}_{X}^{1}\left(\lambda_{i+1}^{\prime}\right) \backslash\{0\}$ such that, if $\mathcal{D}_{i+1}^{X}(f) \neq 0$, then equality holds in (9.39) for almost all $p \in M$.

If $i=s$ in (9.38), then, for all $p \in M$ with regular forward trajectory,

$$
\limsup _{\mathcal{T} \rightarrow+\infty} \frac{\log \left|\int_{0}^{\mathcal{T}} f\left(\Phi_{X}(p, \tau)\right) d \tau\right|}{\log \mathcal{T}}=0 .
$$

Proof. The $X$-invariant distributions $\mathcal{D}^{X} \in H_{W}^{-1}(M)$ are related to $S$-invariant distributions $\mathcal{D}^{S} \in H_{q}^{-1}(M)$ by the following formula:

$$
\mathcal{D}^{X}(f)=\mathcal{D}^{S}\left(W^{-1} f\right), \quad f \in H_{W}^{1}(M) .
$$

Let $E_{i}^{+}(q) \subset \mathcal{B}_{q}^{1}(M), i \in\{1, \ldots, s\}$, be the eigenspace, corresponding to the Lyapunov exponent $\lambda_{i}^{\prime}(X)>0$, of the Oseledec's splitting $\left(9.15^{\prime}\right)$ for the restriction of the cocycle $G_{t}^{c}$ on closed currents of order 1 to the connected component $\mathcal{C}_{X}^{(1)}$. Let $C_{i, j}^{+} \in E_{i}^{+}(q), j=1, \ldots, m_{i}(X)$, be a basis for the space of basic currents of order 1 of the horizontal foliation $\mathcal{F}_{q}=\mathcal{F}_{X}$. By Lemma 6.6, the distributions

$$
\mathcal{D}_{i, j}^{S}:=C_{i, j}^{+} \wedge \eta_{T} \in H_{q}^{-1}(M)
$$

are $S$-invariant. Let $\mathcal{D}_{i, j}^{X}$ be the corresponding $X$-invariant distributions given by (9.40) and let $\mathcal{I}_{X}^{1}\left(\lambda_{i}^{\prime}\right), i \in\{1, \ldots, s\}$, be the vector space generated by $\left\{\mathcal{D}_{i, j}^{X} \mid 1 \leq j \leq m_{i}(X)\right\}$. Let $\alpha_{f}:=f \eta_{T}, f \in H^{1}(M)$. By (9.23),

$$
\gamma_{q}^{\mathcal{T}}\left(\alpha_{f}\right)=\int_{0}^{\mathcal{T}} f\left(\Phi_{S}(p, \tau)\right) d \tau
$$


Since, by (9.41), $C_{i, j}^{+}\left(\alpha_{f}\right)=\mathcal{D}_{i, j}^{S}(f)$, it follows from Theorem 9.5 and (9.31) that (9.39) and $\left(9.39^{\prime}\right)$ hold in the case $X=S$ for all functions in $H^{1}(M)$. The $S$-invariant distributions $\mathcal{D}_{i+1}^{S} \in \mathcal{I}_{S}^{1}\left(\lambda_{i+1}^{\prime}\right), 0 \leq i<s$, such that $\mathcal{D}_{i+1}^{S}(f) \neq 0$ implies equality in (9.39), are constructed as follows. By Theorem 9.5 and Remark $9.5^{\prime}$, for each $i \in\{1, \ldots, s\}$, there exist a sequence of flat cylinders $A_{k}^{(i)} \subset M_{q}$, with area uniformly bounded away from zero, and a sequence $\mathcal{T}_{k}^{(i)}>0$ such that, for any sequence of $q$-regular points $p_{k}^{(i)} \in A_{k}^{(i)}$,

$$
\lim _{k \rightarrow+\infty} \frac{\log \left|\Pi_{q}^{+i}\left(\gamma_{q}^{\mathcal{T}^{(i)}}\left(p_{k}^{(i)}\right)\right)\right|_{-1}}{\log \mathcal{T}_{k}^{(i)}}=\lambda_{i}^{\prime} .
$$

Since $\Pi_{q}^{+i}$ projects onto a finite-dimensional subspace, by passing if necessary to a subsequence, we can define

$$
\mathcal{D}_{i}^{S}:=\lim _{k \rightarrow+\infty} \frac{\Pi_{q}^{+i}\left(\gamma_{q}^{\mathcal{T}_{k}^{(i)}}\left(p_{k}^{(i)}\right)\right)}{\left|\Pi_{q}^{+i}\left(\gamma_{q}^{\mathcal{T}^{(i)}}\left(p_{k}^{(i)}\right)\right)\right|_{-1}} \in \mathcal{I}_{S}^{1}\left(\lambda_{i}^{\prime}\right) .
$$

By $\left(9.32^{\prime}\right)$ the limit in $\left(9.43^{\prime}\right)$ does not depend on the choice of the sequence of points $p_{k}^{(i)} \in A_{k}^{(i)}$. As in (9.29) we define

$$
A_{q}^{(i)}:=\cap_{N \in \mathbb{N}} \cup_{k \geq N} A_{k}^{(i)} .
$$

Since the area of the sets $A_{k}^{(i)}$ is uniformly bounded away from zero, $A_{q}^{(i)}$ has positive measure. By Theorem 9.5, (9.32'), (9.42), (9.43) and $\left(9.43^{\prime}\right)$, for all $q$-regular $p \in A_{q}^{(i)}$, there exists a sequence $\mathcal{T}_{k}:=\mathcal{T}_{k}(p)>0$ such that, if $f \in H^{1}(M)$ and $\mathcal{D}^{S}(f)=0$ for all $\mathcal{D}^{S} \in \mathcal{I}_{S}^{1}\left(\lambda_{1}^{\prime}\right) \oplus \cdots \oplus \mathcal{I}_{S}^{1}\left(\lambda_{i-1}^{\prime}\right)$,

$$
\frac{1}{\left|\Pi_{q}^{+1}\left(\gamma_{q}^{\mathcal{T}_{k}}(p)\right)\right|_{-1}} \int_{0}^{\mathcal{T}_{k}} f\left(\Phi_{S}(p, \tau)\right) d \tau \rightarrow \mathcal{D}_{i}^{S}(f)
$$

Since $A_{q}^{(i)}$ has positive measure and, for almost all $q \in \mathcal{M}_{\kappa}^{(1)}$, the flow $\Phi_{S}$ is ergodic (see [35], [43]), (9.45) holds for almost all $p \in M_{q}$ along a sequence $\mathcal{T}_{k}:=\mathcal{T}_{k}(p)$. We have therefore completed the proof of Theorem 9.6 in the case $X=S$.

Let $f \in H_{W}^{1}(M)$, then by definition $W^{-1} f \in H^{1}(M)$. Let $\gamma_{X}^{\mathcal{T}}(p)$ be the forward $X$-orbit of the (generic) point $p \in M$ up to time $\mathcal{T}>0$. We have

$$
\begin{aligned}
& \int_{0}^{\mathcal{T}} f\left(\Phi_{X}(p, \tau)\right) d \tau=\int_{\gamma_{X}^{\mathcal{T}}(p)} W^{-1} f \eta_{T} \\
& \int_{0}^{\mathcal{T}} W\left(\Phi_{X}(p, \tau)\right) d \tau=L_{q}\left(\gamma_{X}^{\mathcal{T}}(p)\right)
\end{aligned}
$$


In addition, by the Birkhoff ergodic theorem, for almost all $p \in M$,

$$
\lim _{\mathcal{T} \rightarrow+\infty} \frac{1}{\mathcal{T}} \int_{0}^{\mathcal{T}} W\left(\Phi_{X}(p, \tau)\right) d \tau=\int_{M} W \omega=\int_{M} \omega_{q}=1
$$

Hence Theorem 9.6 for a vector field $X=W S$ follows from the case $X=S$ proved above.

We conclude the paper by listing some related questions we have left unanswered:

Question 9.7. Does the Kontsevich-Zorich cocycle have a simple Lyapunov spectrum, with respect to the canonical absolutely continuous invariant measure? The affirmative answer, conjectured by M. Kontsevich and A. Zorich in the joint paper [38], is strongly supported by the numerical evidence reported in [78], [79], [81], [38]. Our paper proves the conjecture in the case of $g=2$. It is unclear to the author whether similar methods can yield a proof in the case of genus $g \geq 3$.

Question 9.8. Is the Kontsevich-Zorich cocycle non-uniformly hyperbolic (or has it simple Lyapunov spectrum) with respect to other invariant ergodic measures of the Teichmüller flow? In particular, it would be interesting, as J. Smillie suggested to the author, to know the answer for periodic Teichmüller disks such as those constructed by W. Veech in [67], which correspond to a class of rational triangular billiards. By the methods developed in this paper, a preliminary step would be to answer the question whether the support of the invariant measure $\mu$ under consideration is contained in the determinant locus. In case the answer to the latter question turns out to be negative, it would then follow at least that $\lambda_{2}^{\mu}>0$ (Theorem 3.3). It seems likely that in the case of Veech surfaces, other known methods would be more effective. The methods described in [21] can probably be applied to prove that the Lyapunov spectrum is simple. We owe this remark to A. Eskin.

Question 9.9. Do there exist basis $\left\{C_{1}^{ \pm}, \ldots, C_{g}^{ \pm}\right\} \subset \mathcal{B}_{ \pm q}^{1}(M)$ of basic currents of order 1 for the horizontal, respectively the vertical, measured foliation $\mathcal{F}_{ \pm q}$ at an Oseledec regular point $q \in \mathcal{M}_{\kappa}^{(1)}$ with the property that in fact $C_{i}^{ \pm} \in \mathcal{H}_{q}^{\lambda_{i}-1}(M)$, the dual Sobolev space of currents with negative exponent $\lambda_{i}-1$ ? This question was formulated independently by M. Kontsevich when we told him about the results of Section 8.1. According to Lemma 8.2, this question can be asked at any regular $q \in \mathcal{M}_{\kappa}^{(1)}$ at which the Lyapunov exponents $\lambda_{1}, \ldots, \lambda_{g}$ are non-zero. A partial form of the question can also be asked in the case that, for any $k \in\{1, \ldots, g\}$, the exponents $\lambda_{1}, \ldots, \lambda_{k}$ are non-zero and $\lambda_{k+1}=0$. In the case $k=1$, we remark that we can choose 
$C_{1}^{+}:=\eta_{S}$ and $C_{1}^{-}:=\eta_{T}$, which are smooth currents. Hence we indeed have that $C_{1}^{ \pm} \in \mathcal{H}_{q}^{\lambda_{1}-1}(M)=\mathcal{H}_{q}^{0}(M)$. An affirmative answer in the case $k>1$ could possibly be obtained by strengthening the proof of Lemma 8.2.

Question 9.10. What is the dynamical significance, if any, of basic currents which are of order $l>1$ ? We have proved in Section 7.1 that all cohomology classes in a codimension 1 subspace of $H^{1}(M, \mathbb{R})$ can be represented by basic currents of order higher than 1. A natural speculation is that they are related to lower order (polynomial, sub-polynomial?) deviations of ergodic averages for sufficiently smooth functions.

Princeton University, Princeton, NJ

E-mail address: gforni@math.princeton.edu

Current address: NORTHWESTERN UNIVERSITY

E-mail address: gforni@math.northwestern.edu

\section{REFERENCES}

[1] R. Adams, Sobolev Spaces. Pure and Applied Mathematics 65, Academic Press, New York-London, 1975.

[2] R. Barre and A. El Kacimi Alaoui, Foliations, in Handbook of Differential Geometry (F. Dillen and L. Verstraelen, eds.), North-Holland, Amsterdam, to appear.

[3] C. Berenstein and R. Gay, Complex Variables. An Introduction, Grad. Texts in Math. 125, Springer-Verlag, New York, 1991.

[4] M. Berger, P. Gauduchon, and E. Mazet, Le Spectre d'une Variété Riemannienne, Lecture Notes in Math. 194, Springer-Verlag, New York, 1971.

[5] L. BERs, Spaces of degenerating Riemann surfaces, in Discontinuous Groups and Riemann Surfaces (Univ. Maryland, College Park, Md., 1973), Ann. of Math. Studies 79, 43-55, Princeton Univ. Press, Princeton, NJ, 1974.

[6] _ Finite-dimensional Teichmüller spaces and generalizations, Bull. A. M. S. 5 (1981), 131-172.

[7] M. Burger, Horocycle flow on geometrically finite surfaces, Duke Math. J. 61 (1990), 779-803.

[8] I. Chavel, Riemannian Geometry - A Modern Introduction, Cambridge Tracts in Math. 108, Cambridge Univ. Press, Cambridge, 1993.

[9] J. Cheeger, A lower bound for the smallest eigenvalue of the Laplacian, in Problems in Analysis (Papers dedicated to Salomon Bochner, 1969), 195-199, Princeton Univ. Press, Princeton, NJ, 1970.

[10] M. Denker, The central limit theorem for dynamical systems, in Dynamical Systems and Ergodic Theory (Warsaw, 1986), Banach Center Publ. 23, 33-62, PWN, Warsaw, 1989.

[11] G. De Rham, Varietés Differentiables. Formes, courants, formes harmoniques (French). Troisime édition revue et augmentée. Publications de l'Institut de Mathematique de l'Université de Nanacago, III. Actualités Scientifiques et Industrielles, $\mathbf{1 2 2 2 b}$, Hermann, Paris, 1973.

[12] A. Eskin and H. Masur, Asymptotic formulas on flat surfaces, Ergodic Theory Dynam. Systems 21 (2001), 443-478.

[13] H. M. Farkas and I. Kra, Riemann Surfaces (second edition), Grad. Texts in Math. 71, Springer-Verlag, New York, 1992. 
[14] A. Fathi, F. Laudenbach, and V. Poénaru, Travaux de Thurston sur les surfaces (French). Séminaire Orsay. Preprint of Travaux de Thurston sure les surfaces, Soc. Math. France, Paris, 1979. Astérisque 66-67 (1991). Société Mathématique de France, Paris, 1991.

[15] J. D. FAY, Theta Functions on Riemann Surfaces, Lecture Notes in Math. 352, SpringerVerlag, Berlin-New York, 1973.

[16] B. R. FAYAD, Reparamétrage de flots irrationnels sur le tore (English), Thèses de Doctorat, École Polytechnique, Paris, Juin 2000.

[17] G. ForNI, The cohomological equation for area-preserving flows on compact surfaces, Electron. Res. Announc. of the A. M. S. 1 (1995), 114-123.

[18] Solutions of the cohomological equation for area-preserving flows on compact surfaces of higher genus, Ann. of Math. 146 (1997), 295-344.

[19] B. Fristedt and L. Gray, A Modern Approach to Probability Theory, Probab. Appl., Birkhäuser, Boston, 1997.

[20] F. Gardiner, Teichmüller Theory and Quadratic Differentials, John Wiley \& Sons, Inc., New York, 1987.

[21] I. YA. Gol'DSherd and G. A. MARGulis, Lyapunov exponents of a product of random matrices (Russian), Uspekhi Mat. Nauk 44 (1989), no. 5(269), 13-60 (English translation: Russian Math. Surveys 44 (1989), no. 5, 11-71).

[22] W. H. Gottschalk and G. A. Hedlund, Topological dynamics, A. M. S. Colloq. Publ. 36, A. M. S. Providence RI, 1955.

[23] P. A. Griffiths, Periods of integrals on algebraic manifolds: summary of main results and discussion of open problems, Bull. A.M.S. 76 (1970), 228-296.

[24] S. Helgason, Groups and Geometric Analysis. Integral Geometry, Invariant Differential Operators, and Spherical Functions, Pure and Appl. Math. 113, Academic Press, Inc., Orlando, Florida, 1984.

[25] M.-R. Herman, Sur la conjugaison différentiable des difféomorphismes du cercle à des rotations (French), IHES Publ. Math. 49 (1979), 5-233.

[26] Une méthode pour minorer les exposants de Lyapunov et quelques exemples montrant le caractère local d'un théorème d'Arnol'd et de Moser sur le tore de dimension 2 (French), Comment. Math. Helv. 58 (1983), 453-502.

[27] J. Hubbard and H. Masur, Quadratic differentials and foliations, Acta Math. 142 (1979), $221-274$.

[28] Y. Imayoshi and M. Taniguchi, An Introduction to Teichmüller Spaces, Springer-Verlag, Tokyo, 1992.

[29] A. KАток, Invariant measures of flows on orientable surfaces (Russian), Dokl. Akad. Nauk SSSR 211 (1973), 775-778 (English translation: Sov. Math. Dokl. 14 (1973), 1104-1108).

[30] Interval exchange transformations and some special flows are not mixing, Israel J. Math. 35 (1980), 301-310.

[31] _ Infinitesimal Lyapunov functions, invariant cone families and stochastic properties of smooth dynamical systems (with the collaboration of K. Burns), Ergod. Theory Dynam. Systems 14 (1994), 757-785.

[32] A. Katok and B. Hasselblatt, Introduction to the Modern Theory of Dynamical Systems, Cambridge Univ. Press, Cambridge, 1995.

[33] — Handbook of Dynamical Systems, Vol. 1, Survey I, Principal Structures, to appear.

[34] M. S. Keane and G. Rauzy, Stricte ergodicité des échanges d'intervalles (French), Math. Z. 174 (1980), 203-212.

[35] S. Kerckhoff, H. Masur, and J. Smillie, Ergodicity of Billiard Flows and Quadratic Differentials, Ann. of Math. 124 (1986), 293-311.

[36] A. Ya. Khinchin, Continued Fractions, The Univ. of Chicago Press, Chicago, Ill.-London, 1964. 
[37] A. V. KočErgin, Mixing in special flows over a rearrangement of segments and in smooth flows on surfaces (Russian), Mat. Sb. (N.S.) 96(138) (1975), 471-502, 504 (English translation: Math. USSR-Sb. 25 (1975), 441-469).

[38] M. Kontsevich, Lyapunov exponents and Hodge theory, in The Mathematical Beauty of Physics (Saclay, 1996), Adv. Ser. Math. Phys. 24, 318-332, World Sci. Publ., River Edge, NJ, 1997.

[39] M. Kontsevich and A. Zorich, Connected components of the moduli space of abelian differentials with prescribed singularities, preprint.

[40] S. Lang, Differentiable Manifolds, Springer-Verlag, New York, 1985.

[41] R. MaÑé, Ergodic Theory and Differentiable Dynamics, Ergeb. Math. Grenzgeb. 8, Springer-Verlag, Berlin, 1987.

[42] H. MAsur, Extension of the Weyl-Petersson metric to the boundary of the Teichmüller space, Duke Math. J. 43 (1976), 623-635.

[43] Interval exchange transformations and measured foliations, Ann. of Math. 115 (1982), 169-200.

[44] Logarithmic law for geodesics in moduli space, in Mapping Class Groups and Moduli Spaces of Riemann Surfaces (Göttingen, 1991/Seattle, WA, 1991), Contemp. Math. 150, 229-245, A. M. S., Providence, RI, 1993.

[45] H. Masur and J. Smillie, Hausdorff dimension of sets of nonergodic measured foliations, Ann. of Math. 134 (1991), 455-543.

[46] S. NAG, The Complex Analytic Theory of Teichmüller Spaces, John Wiley \& Sons, Inc., New York, 1988.

[47] I. Nikolaev and E. Zhuzhoma, Flows on 2-dimensional Manifolds. An Overview, Lectures Notes in Math. 1705, Springer-Verlag, Berlin, 1999.

[48] S. P. Novikov, The Hamiltonian formalism and a multivalued analogue of Morse theory (Russian), Uspekhi Mat. Nauk 37 (1982), 3-49 (English translation: Russian Math. Surveys 37 (1982), 1-56).

[49] V. I. Oseledec, A multiplicative ergodic theorem. Characteristic Ljapunov, exponents of dynamical systems (Russian), Trudy Moskov Mat. Obšč 19 (1968), 179-210 (English translation: Trans. Moscow Math. Soc. 19 (1968), 197-231).

[50] K. Petersen, Ergodic Theory, Cambridge Studies in Adv. Math. 2, Cambridge Univ. Press, Cambridge, 1989.

[51] M. Ratner, Rigidity of time changes for horocycle flows, Acta Math. 156 (1986), 1-32.

[52] The rate of mixing for geodesic and horocycle flows, Ergod. Theory Dynamic. Systems 7 (1987), 267-288.

[53] B. L. Reinhart, Harmonic integrals on foliated manifolds, Amer. J. Math. 81 (1959), 529-536.

[54] Differential Geometry of Foliations, Springer-Verlag, New York, 1983.

[55] K.-U. Schaumlöffel, Multiplicative ergodic theorems in infinite dimensions, in Lyapunov Exponents (Oberwolfach, 1990), Lecture Notes in Math. 1486, 187-195, Springer-Verlag, Berlin, 1991.

[56] L. Schwartz, Théorie des Distributions (French), Publ. de l'Institut de Math. de l'Université de Strasbourg, No. IX-X, Nouvelle édition, entiérement corrigée, refondue et augmentée, Hermann, Paris, 1966.

[57] S. Schwartzman, Asymptotic cycles, Ann. of Math. 66 (1957), 270-284.

[58] K. Strebel, Quadratic Differentials, Ergeb. Math. Grenzgeb. 5, Springer-Verlag, Berlin, 1984.

[59] D. Sullivan, The Dirichlet problem at infinity for a negatively curved manifold, J. Differential Geom. 18 (1983), 723-732.

[60] M. Taniguchi, A note on the second variational formulas of functionals on Riemann surfaces, Kodai Math. J. 12 (1989), 283-295. 
[61] M. TAniguchi, The behavior of the extremal length function on arbitrary Riemann surface, in Prospects in Complex Geometry (Katata and Kyoto, 1989), Lecture Notes in Math. 1468, 160-169, Springer-Verlag, Berlin, 1991.

[62] W. Thurston, On the geometry and dynamics of diffeomorphisms of surfaces, Bull. A. M. S. 19 (1988), 417-431.

[63] P. Tondeur, Geometry of Foliations, Monographs in Math. 90, Birkhäuser Verlag, Basel, 1997.

[64] A. Tromba, Teichmüller Theory in Riemannian Geometry, Lectures Math. ETH Zürich, Birkhaüser Verlag, Basel, 1992.

[65] W. A. Veech, Gauss measures for transformations on the space of interval exchange maps, Ann. of Math. 115 (1982), 201-242.

[66] The Teichmüller geodesic flow, Ann. of Math. 124 (1986), 441-530.

[67] Teichmüller curves in moduli space, Eisenstein series and an application to triangular billiards, Invent. Math. 97 (1989), 553-583.

[68] Moduli spaces of quadratic differentials, J. Analyse Math. 55 (1990), 117-171.

[69] Siegel measures, Ann. of Math. 148 (1998), 895-944.

[70] R. O. Wells, Differential Analysis on Complex Manifolds (second edition), Grad. Texts in Math. 65, Springer-Verlag, New York-Berlin, 1980.

[71] A. Yamada, Precise variational formulas for abelian differentials, Kodai Math. J. 3 (1980), $114-143$.

[72] S. YAmadA, Weil-Petersson convexity of the energy functional on classical and universal Teichmüller spaces, J. Differential Geom. 51 (1999), 35-96.

[73] S. T. YAU, Isoperimetric constants and the first eigenvalue of a compact Riemannian manifold, Ann. Sci. École Norm. Sup. 8 (1975), 487-507.

[74] L.-S. Young, Entropy of continuous flows on compact 2-manifolds, Topology 16 (1977), 469-471.

[75] Statistical properties of dynamical systems with some hyperbolicity, Ann. of Math. 147 (1998), 585-650.

[76] Developments in chaotic dynamics, Notices A. M. S. 45, no. 10 (1998), 13181328.

[77] A. Zemljakov and A. Katok, Topological transitivity of billiards in polygons (Russian), Mat. Zametki 18 (1975), 291-300 (English translation: Math. Notes 18 (1975), 760-764).

[78] A. Zorich, Asymptotic flag of an orientable measured foliation on a surface, in Geometric Study of Foliations (Tokyo, 1993), 479-498, World Sci. Publ., River Edge, NJ, 1994.

[79] - Finite Gauss measure on the space of interval exchange transformations. Lyapunov exponents, Ann. Inst. Fourier (Grenoble) 46 (1996), 325-370.

[80] Deviation for interval exchange transformations, Ergod. Theory Dynam. Systems 17 (1997), 1477-1499.

[81] A. Zorich, On hyperplane sections of periodic surfaces, in Solitons, Geometry and Topology: on the Crossroad, A. M. S. Transl. 179, 173-189, A. M. S. Providence, RI, 1997.

[82] How do the leaves of a closed 1-form wind around a surface?, in Pseudoperiodic Topology, A. M. S. Transl. 197, 135-178, A. M. S., Providence, RI, 1999.

(Received November 24, 1999)

(Revised May 16, 2000) 